\title{
Origins of Stereoselectivity in Peptide-Catalyzed Kinetic Resolution of Alcohols
}

\author{
Rong-Zhen Liao, ${ }^{* 1}$ Stefano Santoro, ${ }^{* 2}$ Martin Gotsev, ${ }^{3}$ Tommaso Marcelli ${ }^{3}$ and \\ Fahmi Himo *3
}

1) Key Laboratory of Material Chemistry for Energy Conversion and Storage, Ministry of Education, School of Chemistry and Chemical Engineering, Huazhong University of Science and Technology, Wuhan 430074, People's Republic of China.

E-mail:rongzhen@hust.edu.cn

2) Department of Chemistry, Biology and Biotechnology, University of Perugia, Via Elce di Sotto 8, 06123 Perugia, Italy.

E-mail: stefano.santoro@unipg.it

3) Department of Organic Chemistry, Arrhenius Laboratory, Stockholm University, SE-106

91 Stockholm, Sweden.

E-mail:fahmi.himo@su.se

\section{Contents}

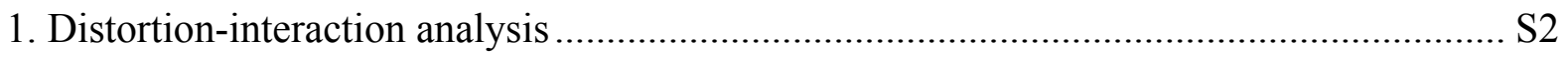

2. Conformer definition for the tetrapeptide catalyst study …......................................... S3

3. Geometrical features of optimized TSs for the tetrapeptide catalyst ............................... S4

4. Relative TS energies for the tetrapeptide catalyst with different functionals ................... S5

5. Structures and Cartesian coordinates of optimized stationary points .............................. S6 


\section{Distortion-interaction analysis}

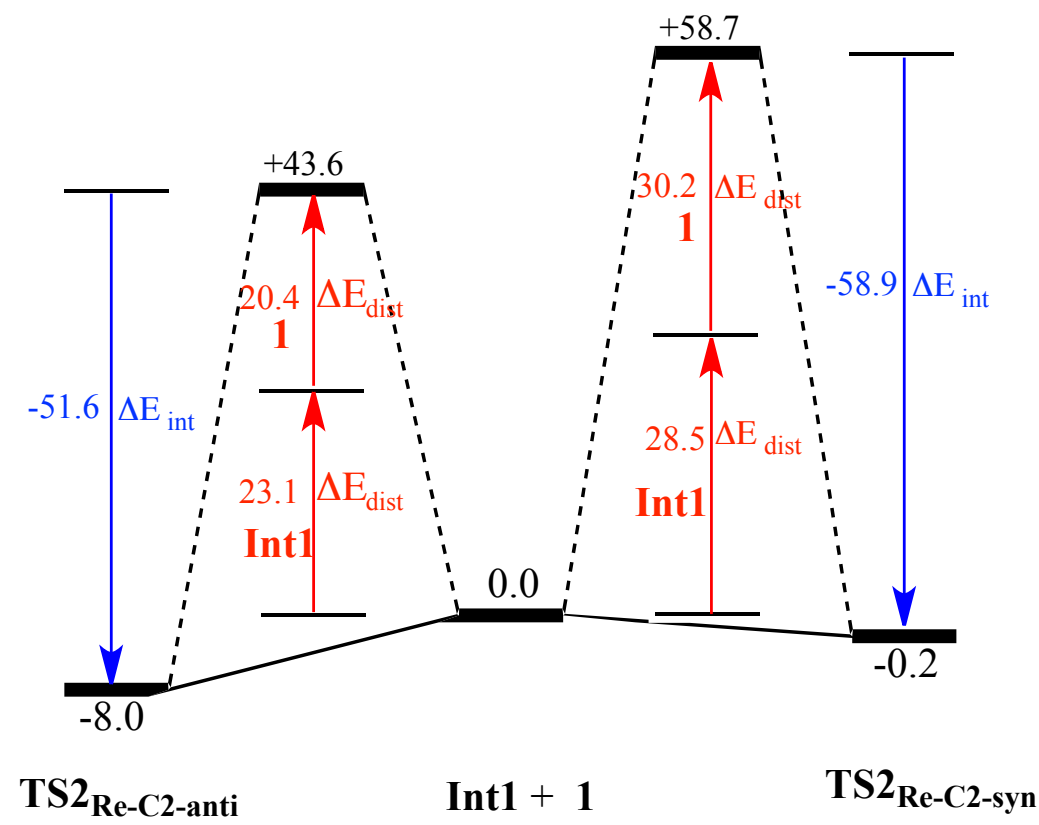

Distortion-interaction comparison between the syn and anti versions of the TS2 $\mathbf{R e}_{\mathbf{R e}-\mathbf{C}}$ transition state. Energies are calculated in the gas phase at the B3LYP/6-31G(d,p) level. Alcohol 1 is distorted by ca $10 \mathrm{kcal} / \mathrm{mol}$ more in the syn TS compared to the anti counterpart. 


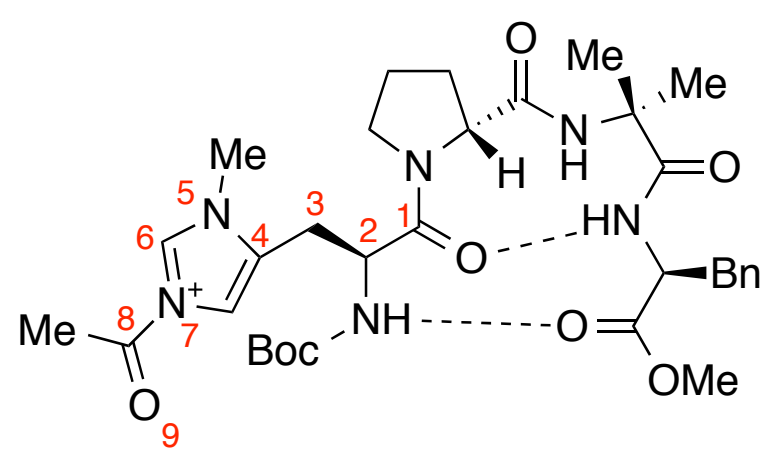

A: dihedral angle C1-C2-C3-C4

$$
\mathrm{A} 1 \approx+60^{\circ} ; \mathrm{A} 2 \approx+180^{\circ} ; \mathrm{A} 3 \approx-60^{\circ}
$$

B: dihedral angle C2-C3-C4-N5

$$
\mathrm{B} 1 \approx-90^{\circ} ; \mathrm{B} 2 \approx+90^{\circ}
$$

C: dihedral angle C6-N7-C8-O9

$$
\mathrm{C} 1 \approx+180^{\circ} ; \mathrm{C} 2 \approx 0^{\circ}
$$




\section{Geometrical features of optimized TSs for the tetrapeptide catalyst}

Table S1. Dihedral angles $\boldsymbol{A}, \boldsymbol{B}$ and $\boldsymbol{C}$ (in degrees) of the optimized transition state structures.

\begin{tabular}{|c|c|c|c|c|c|c|c|c|}
\hline \multirow{2}{*}{ TS } & \multicolumn{4}{|c|}{$(R, R)-1$} & \multicolumn{4}{|c|}{$(S, S)-1$} \\
\hline & Mode & $\mathbf{A}$ & B & C & Mode & $\mathbf{A}$ & B & C \\
\hline $\mathrm{A} 1 \mathrm{~B} 1 \mathrm{C} 1 \mathrm{Si}$ & III & 66.0 & -112.8 & -152.8 & III & 65.4 & -113.1 & -162.7 \\
\hline $\mathrm{A} 2 \mathrm{~B} 1 \mathrm{C} 1 \mathrm{Si}$ & III & -162.3 & -72.5 & -151.7 & III & -165.2 & -73.3 & -162.7 \\
\hline A3B1C1Si & III & -54.8 & -88.2 & -159.3 & III & -77.0 & -119.1 & -163.5 \\
\hline $\mathrm{A} 1 \mathrm{~B} 2 \mathrm{C} 1 \mathrm{Si}$ & III & 78.5 & 88.6 & -149.1 & III & 73.5 & 88.4 & -163.7 \\
\hline $\mathrm{A} 2 \mathrm{~B} 2 \mathrm{C} 1 \mathrm{Si}$ & III & -176.9 & 92.5 & -172.2 & III & 173.5 & 89.2 & -162.8 \\
\hline $\mathrm{A} 3 \mathrm{~B} 2 \mathrm{C} 1 \mathrm{Si}$ & I & -51.2 & 168.7 & 174.0 & I & -65.3 & -177.5 & -171.2 \\
\hline $\mathrm{A} 1 \mathrm{~B} 1 \mathrm{C} 2 \mathrm{Si}$ & I & 52.4 & -125.1 & 18.3 & III & 60.8 & -149.2 & 5.7 \\
\hline $\mathrm{A} 2 \mathrm{~B} 1 \mathrm{C} 2 \mathrm{Si}$ & III & -179.7 & -68.2 & 2.3 & III & 172.2 & -94.3 & 4.6 \\
\hline $\mathrm{A} 3 \mathrm{~B} 1 \mathrm{C} 2 \mathrm{Si}$ & I & -31.9 & -68.2 & 26.8 & I & -52.5 & -87.6 & 13.4 \\
\hline $\mathrm{A} 1 \mathrm{~B} 2 \mathrm{C} 2 S i$ & II & 73.2 & 98.5 & 25.2 & II & 74.1 & 102.2 & 21.4 \\
\hline $\mathrm{A} 2 \mathrm{~B} 2 \mathrm{C} 2 \mathrm{Si}$ & III & 174.8 & 88.0 & 9.0 & III & 174.4 & 89.4 & 9.6 \\
\hline $\mathrm{A} 3 \mathrm{~B} 2 \mathrm{C} 2 \mathrm{Si}$ & III & -79.7 & 106.5 & -7.0 & III & -62.3 & 115.3 & 8.1 \\
\hline $\mathrm{A} 1 \mathrm{~B} 1 \mathrm{C} 1 \mathrm{Re}$ & III & 62.6 & -115.3 & 163.6 & III & 76.3 & -103.0 & 170.8 \\
\hline $\mathrm{A} 2 \mathrm{~B} 1 \mathrm{C} 1 \mathrm{Re}$ & III & 177.4 & -114.1 & 158.8 & III & 169.6 & 178.0 & 159.3 \\
\hline $\mathrm{A} 3 \mathrm{~B} 1 \mathrm{C} 1 \mathrm{Re}$ & III & -48.5 & -86.5 & 161.6 & III & -73.2 & -130.4 & 147.5 \\
\hline $\mathrm{A} 1 \mathrm{~B} 2 \mathrm{C} 1 \mathrm{Re}$ & III & 75.5 & 87.6 & 164.3 & III & 75.2 & 90.4 & 159.2 \\
\hline $\mathrm{A} 2 \mathrm{~B} 2 \mathrm{C} 1 R e$ & III & 176.8 & 97.2 & 163.6 & III & 175.6 & 91.7 & 146.1 \\
\hline $\mathrm{A} 3 \mathrm{~B} 2 \mathrm{C} 1 R e$ & III & -65.3 & 119.6 & 168.9 & III & -61.7 & 122.3 & -178.8 \\
\hline $\mathrm{A} 1 \mathrm{~B} 1 \mathrm{C} 2 \mathrm{Re}$ & III & 63.6 & -158.7 & -11.3 & III & 64.4 & -164.4 & -10.7 \\
\hline $\mathrm{A} 2 \mathrm{~B} 1 \mathrm{C} 2 \mathrm{Re}$ & III & -179.0 & -74.4 & -13.2 & III & -166.2 & -74.9 & 0.9 \\
\hline $\mathrm{A} 3 \mathrm{~B} 1 \mathrm{C} 2 \mathrm{Re}$ & II & -65.8 & -109.1 & -11.5 & II & -67.5 & -107.8 & -7.6 \\
\hline $\mathrm{A} 1 \mathrm{~B} 2 \mathrm{C} 2 R e$ & II & 70.8 & 100.5 & -1.8 & II & 69.9 & 102.1 & -2.2 \\
\hline $\mathrm{A} 2 \mathrm{~B} 2 \mathrm{C} 2 \mathrm{Re}$ & III & 159.9 & 92.1 & -10.2 & \multicolumn{4}{|c|}{ converges to $\mathrm{A} 1 \mathrm{~B} 2 \mathrm{C} 2 \mathrm{Re}$} \\
\hline $\mathrm{A} 3 \mathrm{~B} 2 \mathrm{C} 2 \mathrm{Re}$ & III & -67.1 & 118.1 & -3.7 & III & -68.1 & 94.1 & -18.9 \\
\hline
\end{tabular}

Note that some of the dihedral angles of the optimized structures, especially dihedral angle $\mathbf{B}$, deviate from the typical range. To avoid confusion, the transition states are named with respect to the starting structure for the geometry optimization. 


\section{Relative TS energies for the tetrapeptide catalyst with different functionals}

Table S2. Calculated relative free energies (in $\mathrm{kcal} / \mathrm{mol}$ ) of the transition states for the acylation of $(R, R)-1$ and $(S, S)-1$, relative to A1B1C2Si. The lowest energy in each series is indicated in bold face.

\begin{tabular}{|c|c|c|c|c|c|c|c|c|}
\hline & \multicolumn{4}{|c|}{$(R, R)-1$} & \multicolumn{4}{|c|}{$(S, S)-1$} \\
\hline & Mode & B3LYP-D2 & M06 & B3LYP & Mode & B3LYP-D2 & M06 & B3LYP \\
\hline $\mathrm{A} 1 \mathrm{~B} 1 \mathrm{C} 1 \mathrm{Si}$ & III & 5.5 & 4.0 & -2.8 & III & 5.2 & 3.0 & -4.8 \\
\hline $\mathrm{A} 2 \mathrm{~B} 1 \mathrm{C} 1 S i$ & III & 3.9 & 3.5 & -1.2 & III & 4.6 & 3.6 & -2.2 \\
\hline $\mathrm{A} 3 \mathrm{~B} 1 \mathrm{C} 1 S i$ & III & 6.0 & 4.1 & -1.3 & III & 5.8 & 4.0 & -3.5 \\
\hline $\mathrm{A} 1 \mathrm{~B} 2 \mathrm{C} 1 \mathrm{Si}$ & III & 5.6 & 3.7 & -1.2 & III & 2.7 & 1.4 & -5.1 \\
\hline $\mathrm{A} 2 \mathrm{~B} 2 \mathrm{C} 1 S i$ & III & 6.4 & 4.7 & 9.8 & III & 4.8 & 3.8 & -3.2 \\
\hline $\mathrm{A} 3 \mathrm{~B} 2 \mathrm{C} 1 \mathrm{Si}$ & I & 1.4 & -0.2 & 1.3 & I & 9.2 & 7.9 & 6.1 \\
\hline $\mathrm{A} 1 \mathrm{~B} 1 \mathrm{C} 2 \mathrm{Si}$ & I & 0.0 & 0.0 & 0.0 & III & 3.0 & 2.2 & 0.1 \\
\hline $\mathrm{A} 2 \mathrm{~B} 1 \mathrm{C} 2 \mathrm{~S} i$ & III & 5.6 & 4.8 & 6.2 & III & 8.6 & 9.6 & 3.1 \\
\hline $\mathrm{A} 3 \mathrm{~B} 1 \mathrm{C} 2 \mathrm{Si}$ & I & 1.3 & 1.0 & 0.8 & I & 3.5 & 2.7 & 9.8 \\
\hline $\mathrm{A} 1 \mathrm{~B} 2 \mathrm{C} 2 \mathrm{Si}$ & II & 7.6 & 7.3 & 6.2 & II & 6.0 & 4.9 & 2.8 \\
\hline $\mathrm{A} 2 \mathrm{~B} 2 \mathrm{C} 2 \mathrm{Si}$ & III & 6.8 & 6.6 & 3.9 & III & 6.9 & 5.2 & -1.8 \\
\hline $\mathrm{A} 3 \mathrm{~B} 2 \mathrm{C} 2 \mathrm{Si}$ & III & 7.1 & 7.8 & 4.8 & III & 6.9 & 7.5 & 4.3 \\
\hline $\mathrm{A} 1 \mathrm{~B} 1 \mathrm{C} 1 \mathrm{Re}$ & III & 4.5 & 3.3 & -3.6 & III & 7.7 & 8.5 & 2.4 \\
\hline $\mathrm{A} 2 \mathrm{~B} 1 \mathrm{C} 1 \mathrm{Re}$ & III & 5.8 & 4.5 & -2.5 & III & 4.0 & 1.9 & -2.2 \\
\hline $\mathrm{A} 3 \mathrm{~B} 1 \mathrm{C} 1 \mathrm{Re}$ & III & 4.1 & 3.0 & -2.3 & III & 8.1 & 9.0 & 6.6 \\
\hline $\mathrm{A} 1 \mathrm{~B} 2 \mathrm{C} 1 \mathrm{Re}$ & III & 7.3 & 4.5 & -3.3 & III & 5.2 & 3.9 & -1.8 \\
\hline $\mathrm{A} 2 \mathrm{~B} 2 \mathrm{C} 1 R e$ & III & 5.2 & 2.7 & -3.1 & III & 4.3 & 2.4 & -0.3 \\
\hline $\mathrm{A} 3 \mathrm{~B} 2 \mathrm{C} 1 R e$ & III & 3.9 & 3.8 & -1.9 & III & 4.7 & 4.8 & 1.4 \\
\hline $\mathrm{A} 1 \mathrm{~B} 1 \mathrm{C} 2 \mathrm{Re}$ & III & 6.1 & 4.4 & -1.7 & III & 6.8 & 5.7 & 2.8 \\
\hline $\mathrm{A} 2 \mathrm{~B} 1 \mathrm{C} 2 \mathrm{Re}$ & III & 8.0 & 8.1 & 0.9 & III & 7.4 & 6.0 & 3.7 \\
\hline $\mathrm{A} 3 \mathrm{~B} 1 \mathrm{C} 2 \mathrm{Re}$ & II & 5.3 & 4.1 & 2.5 & II & 6.5 & 5.5 & 4.6 \\
\hline $\mathrm{A} 1 \mathrm{~B} 2 \mathrm{C} 2 \mathrm{Re}$ & II & 2.4 & 1.4 & -0.4 & II & 2.6 & 2.8 & 3.2 \\
\hline $\mathrm{A} 2 \mathrm{~B} 2 \mathrm{C} 2 \mathrm{Re}$ & III & 7.4 & 6.4 & 0.7 & \multicolumn{4}{|c|}{ converges to $\mathrm{A} 1 \mathrm{~B} 2 \mathrm{C} 2 \mathrm{Re}$} \\
\hline $\mathrm{A} 3 \mathrm{~B} 2 \mathrm{C} 2 R e$ & III & 10.3 & 12.2 & 9.0 & III & 8.5 & 9.7 & 7.8 \\
\hline
\end{tabular}




\section{Structures and Cartesian coordinates of optimized stationary points}

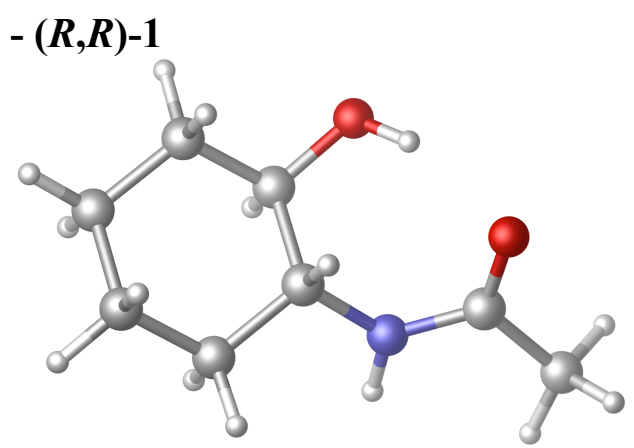

$\begin{array}{llll}\mathrm{C} & -1.33458 & 2.0687 & 0.14115 \\ \mathrm{C} & -2.17329 & 3.01788 & -0.67845 \\ \mathrm{O} & -1.62851 & 1.66381 & 1.2508 \\ \mathrm{C} & 3.04521 & -0.99424 & -0.04317 \\ \mathrm{C} & 3.27052 & 0.4949 & 0.25263 \\ \mathrm{C} & 1.6658 & -1.45034 & 0.44856 \\ \mathrm{C} & 2.13922 & 1.35137 & -0.33559 \\ \mathrm{C} & 0.52876 & -0.59688 & -0.15208 \\ \mathrm{C} & 0.76808 & 0.88648 & 0.15517 \\ \mathrm{O} & -0.21372 & 1.72316 & -0.52294 \\ \mathrm{~N} & -0.78042 & -1.01766 & 0.33323 \\ \mathrm{C} & -1.42232 & -2.09791 & -0.19865 \\ \mathrm{O} & -0.92422 & -2.79474 & -1.07821 \\ \mathrm{C} & -2.79528 & -2.39528 & 0.38661 \\ \mathrm{H} & -2.47008 & 2.53314 & -1.61329 \\ \mathrm{H} & -3.05823 & 3.30446 & -0.11171 \\ \mathrm{H} & -1.59028 & 3.90448 & -0.94252 \\ \mathrm{H} & 2.15401 & 1.28735 & -1.43103 \\ \mathrm{H} & 2.2697 & 2.40753 & -0.07644 \\ \mathrm{H} & 4.23241 & 0.83094 & -0.15016 \\ \mathrm{H} & 3.31707 & 0.64759 & 1.33984 \\ \mathrm{H} & 3.1221 & -1.16665 & -1.12538 \\ \mathrm{H} & 3.82957 & -1.59958 & 0.42483 \\ \mathrm{H} & 1.61709 & -1.38081 & 1.5441 \\ \mathrm{H} & 1.47589 & -2.49321 & 0.18109 \\ \mathrm{H} & 0.53207 & -0.73573 & -1.24051 \\ \mathrm{H} & 0.66491 & 1.05911 & 1.23229 \\ \mathrm{H} & -1.24087 & -0.434 & 1.01962 \\ \mathrm{H} & -3.18565 & -1.59166 & 1.01685 \\ \mathrm{H} & -2.73763 & -3.31257 & 0.98064 \\ \mathrm{H} & -3.49022 & -2.58261 & -0.43515\end{array}$

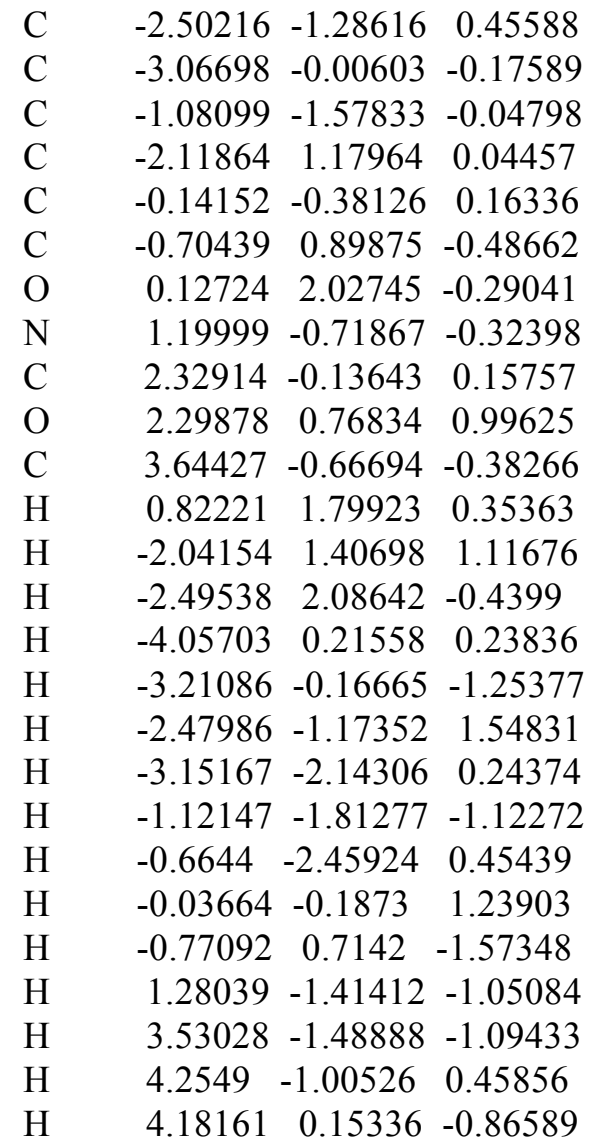

- $(R, R)-2$

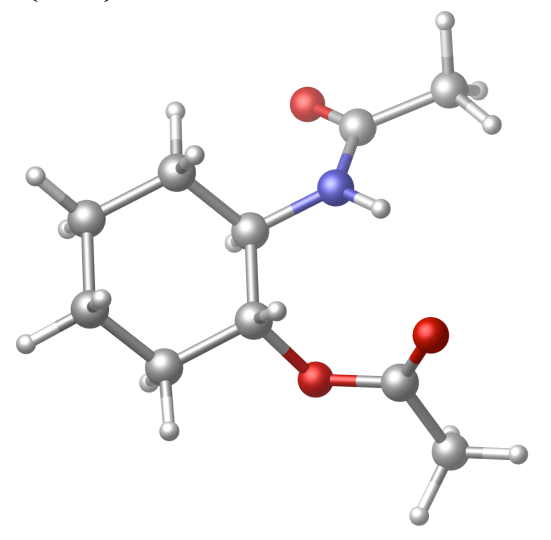

\section{- 1,5-dimethylimidazole}

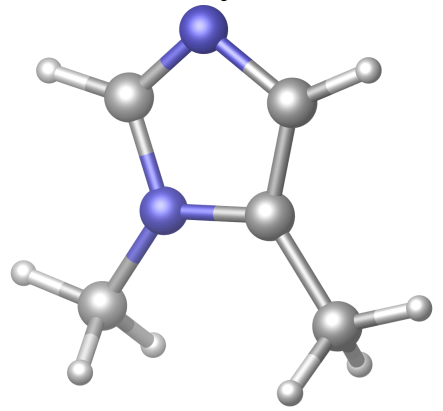

C $\quad-1.64347-1.34671-0.00019$

C $\quad-0.29848-0.69928-0.00036$

$\mathrm{N} \quad-0.11642 \quad 0.67664-0.00173$

$\begin{array}{llll}\text { C } & 0.97461 & -1.22079 & 0.00068\end{array}$

$\begin{array}{llll}\mathrm{C} & 1.23677 & 0.89436 & -0.00044\end{array}$

$\begin{array}{llll}\mathrm{N} & 1.92498 & -0.22478 & 0.00055\end{array}$

C $\quad-1.15358 \quad 1.69061 \quad 0.00103$

$\mathrm{H} \quad-1.52446-2.43271-0.0025$

$\mathrm{H} \quad-2.23801 \quad-1.07852 \quad-0.88288$ 

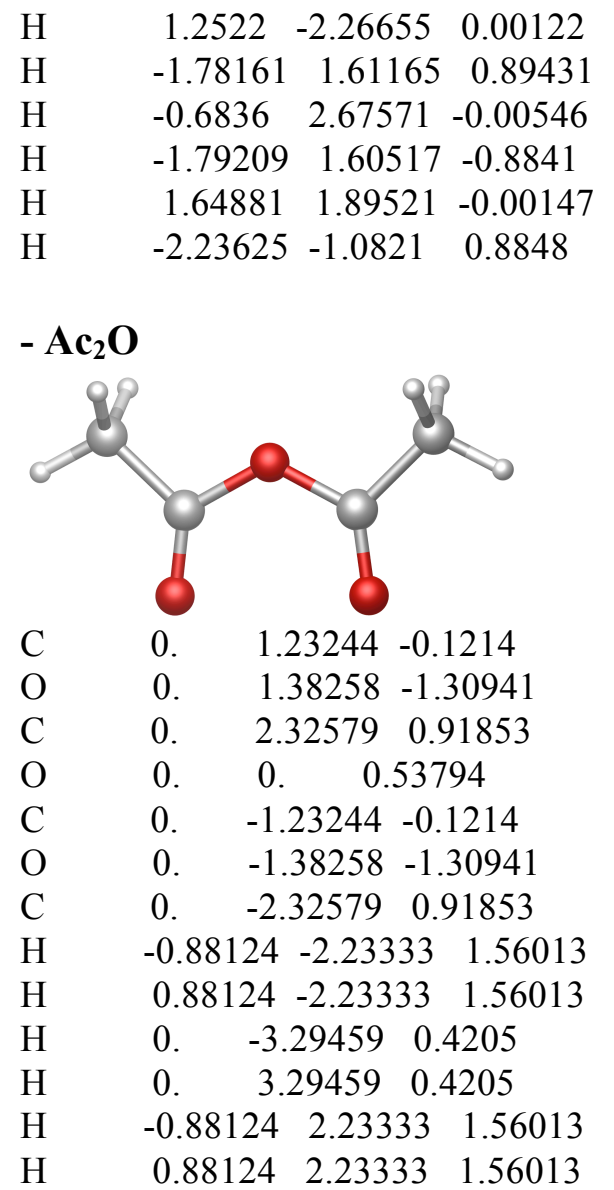

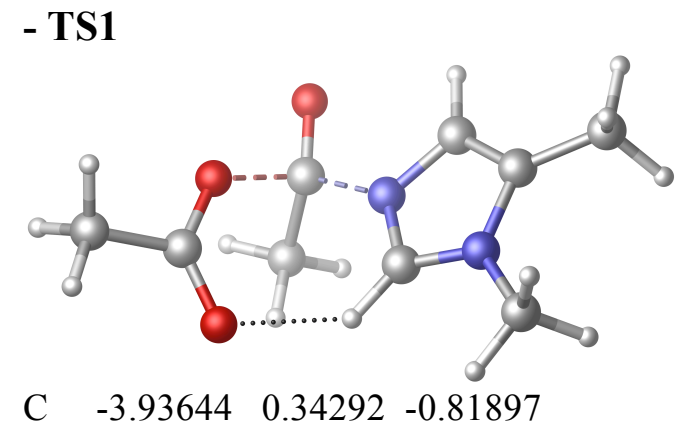

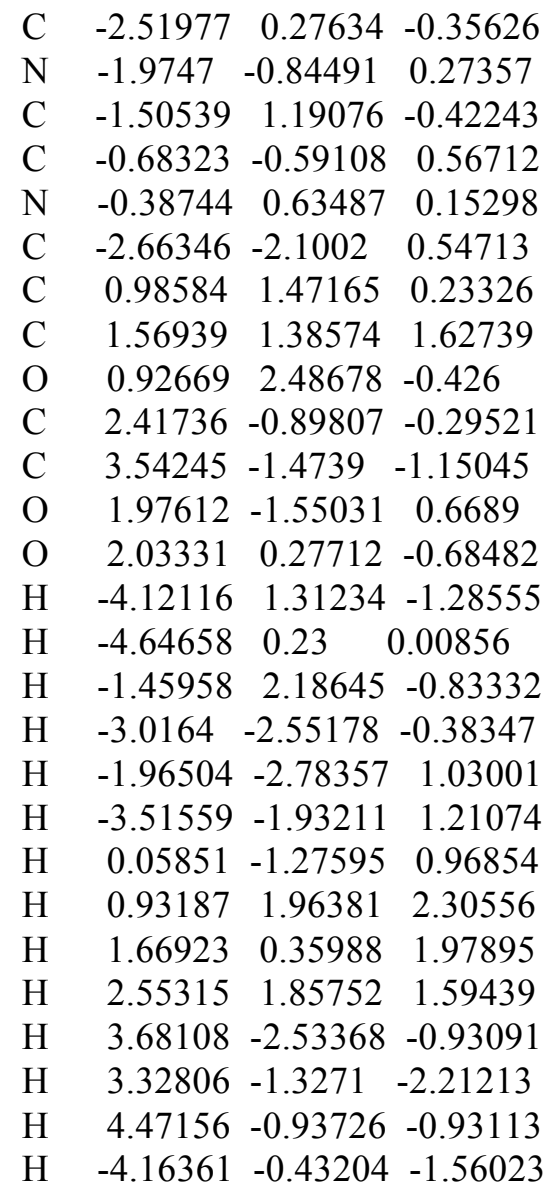

- Int

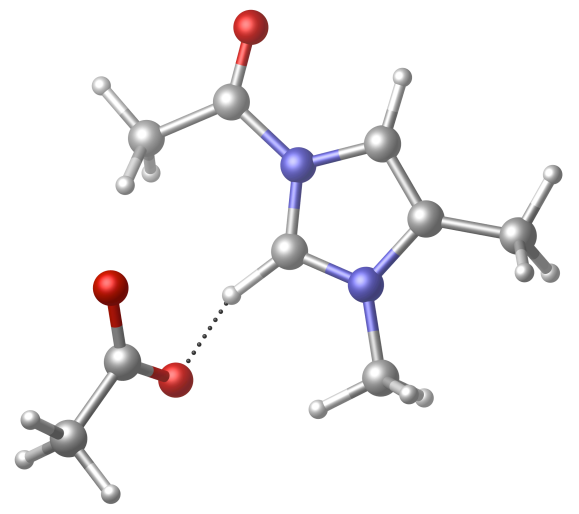

$\begin{array}{llll}\mathrm{C} & -3.30482 & -2.05647 & -0.48478 \\ \mathrm{C} & -2.3013 & -0.99437 & -0.18962 \\ \mathrm{~N} & -0.99854 & -1.27447 & 0.25049 \\ \mathrm{C} & -2.38481 & 0.3598 & -0.26804 \\ \mathrm{C} & -0.31687 & -0.14125 & 0.43561 \\ \mathrm{~N} & -1.14982 & 0.87411 & 0.1222 \\ \mathrm{C} & -0.40316 & -2.59756 & 0.46217 \\ \mathrm{C} & -0.82861 & 2.29635 & 0.10884 \\ \mathrm{C} & 0.55689 & 2.6344 & 0.5447 \\ \mathrm{O} & -1.69418 & 3.05096 & -0.26273 \\ \mathrm{C} & 2.8596 & -0.3019 & -0.17456 \\ \mathrm{C} & 4.22518 & -0.91264 & -0.50081\end{array}$ 


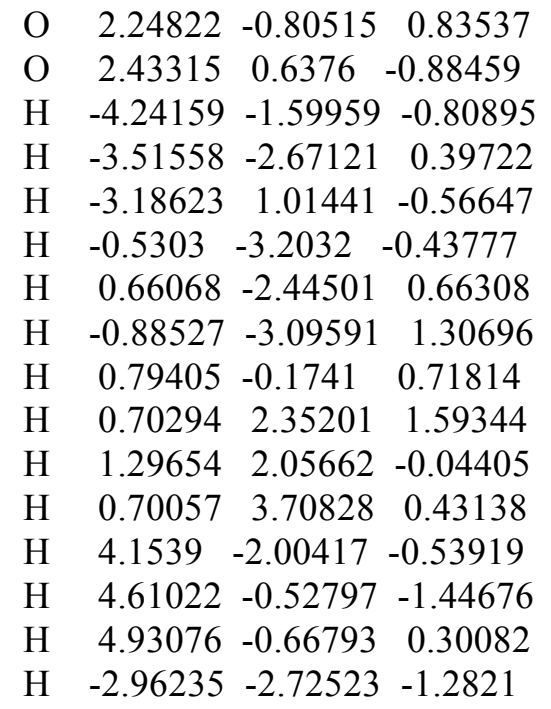

\section{- TS1'}

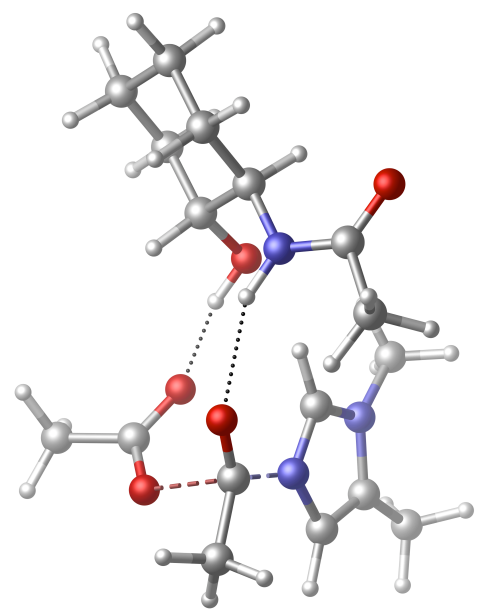

$\begin{array}{llll}\text { C } & 4.50585 & -0.94827 & 2.45321\end{array}$

$\begin{array}{llll}\text { C } & 3.48017 & -0.81644 & 1.37959\end{array}$

$\begin{array}{llll}\mathrm{N} & 2.10361 & -0.87548 & 1.62647\end{array}$

$\begin{array}{llll}\text { C } & 3.61021 & -0.63057 & 0.03621\end{array}$

$\begin{array}{llll}\text { C } & 1.44862 & -0.7188 & 0.47642\end{array}$

N $\quad 2.33659 \quad-0.59456 \quad-0.51262$

C $\quad 1.44186 \quad-0.97135 \quad 2.92777$

C $\quad 1.89236 \quad-0.21115 \quad-1.87824$

C $2.9766 \quad-0.21899 \quad-2.92234$

$\begin{array}{llll}\text { O } & 0.70812 & -0.35442 & -2.11856\end{array}$

C $\quad-5.10883 \quad 0.71659 \quad 0.15522$

$\begin{array}{llll}\text { C } & -4.45908 & 2.05269 & 0.53868\end{array}$

$\begin{array}{llll}\text { C } & -4.1247 & -0.17027 & -0.61865\end{array}$

C $\quad-3.15732 \quad 1.82205 \quad 1.31793$

C $\quad-2.81309 \quad-0.40081 \quad 0.15975$

$\begin{array}{llll}C & -2.1606 & 0.92974 & 0.5627\end{array}$

$\begin{array}{llll}\mathrm{O} & -1.01717 & 0.67543 & 1.37413\end{array}$

N $\quad-1.88139-1.22754 \quad-0.60477$

C $\quad-1.97296-2.59052-0.60156$

O $\quad-2.77279 \quad-3.20382 \quad 0.10069$ $\begin{array}{llll}\text { C } & 1.64712 & 2.47658 & -0.60412\end{array}$

C $\quad 1.59089 \quad 4.00011-0.72279$

$\begin{array}{llll}\text { O } & 1.09463 & 1.9433 & 0.39407\end{array}$

$\begin{array}{lllll}\text { O } & 2.25469 & 1.86296 & -1.53873\end{array}$

$\begin{array}{llll}\text { C } & -1.01204 & -3.31465 & -1.53564\end{array}$

H $\quad 5.50476-0.88647 \quad 2.0172$

H $4.42584-1.906112 .97919$

H $\quad 4.49862 \quad-0.52403 \quad-0.56157$

H $\quad \begin{array}{llll}1.89981 & -0.26114 & 3.6187\end{array}$

H $\quad 0.39244 \quad-0.70581 \quad 2.79039$

H $\quad 1.53098-1.98356 \quad 3.33042$

H $\quad 0.37452 \quad-0.65638 \quad 0.38455$

$\begin{array}{lllll}\mathrm{H} & 3.87832 & 0.29502 & -2.59421\end{array}$

H $3.20786-1.25766 \quad-3.18802$

$\begin{array}{lllll}\mathrm{H} & 2.58426 & 0.29132 & -3.80091\end{array}$

$\begin{array}{llll}\mathrm{H} & 1.75117 & 4.45949 & 0.25619\end{array}$

$\begin{array}{llll}\mathrm{H} & 2.32314 & 4.3702 & -1.4426\end{array}$

H $\quad 0.59059 \quad 4.29227-1.06226$

H $\quad-0.31278 \quad 1.30965 \quad 1.10577$

$\mathrm{H} \quad-3.37703 \quad 1.33543 \quad 2.2776$

$\begin{array}{llll}\mathrm{H} & -2.66543 & 2.77282 & 1.55375\end{array}$

$\begin{array}{llll}\mathrm{H} & -5.14985 & 2.66266 & 1.13274\end{array}$

H $\quad-4.24477 \quad 2.62666 \quad-0.37412$

$\begin{array}{llll}\mathrm{H} & -5.42978 & 0.1936 & 1.06671\end{array}$

$\begin{array}{lllll}\mathrm{H} & -6.01265 & 0.88432 & -0.4423\end{array}$

$\mathrm{H} \quad-3.88415 \quad 0.30053-1.58258$

$\begin{array}{lllll}\mathrm{H} & -4.56808 & -1.14612 & -0.83991\end{array}$

$\begin{array}{llll}\mathrm{H} & -3.04215 & -0.96265 & 1.07229\end{array}$

$\begin{array}{lllll}\mathrm{H} & -1.84367 & 1.44975 & -0.35687\end{array}$

$\mathrm{H} \quad-1.23485-0.78046-1.24646$

H $\quad-0.50998-4.10665-0.97347$

$\mathrm{H} \quad-0.27135 \quad-2.65634-1.99475$

$\mathrm{H} \quad-1.59406 \quad-3.80104 \quad-2.32462$

H $\quad 4.41658 \quad-0.14953 \quad 3.19791$

\section{- Int'}

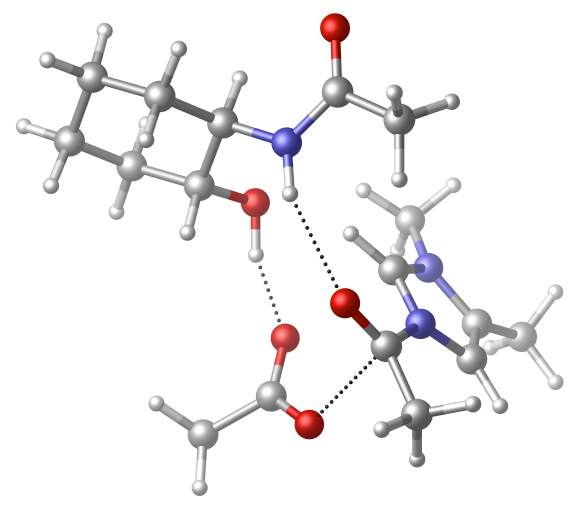

$\begin{array}{llll}\text { C } & 4.58148 & -0.7178 & 2.38789\end{array}$

$\begin{array}{llll}\text { C } & 3.53676 & -0.68809 & 1.32581\end{array}$

$\begin{array}{llll}\mathrm{N} & 2.16359 & -0.68783 & 1.60825\end{array}$

$\begin{array}{llll}\text { C } & 3.64216 & -0.66105 & -0.02909\end{array}$

$\begin{array}{llll}\text { C } & 1.48281 & -0.62722 & 0.46735\end{array}$ 


$\begin{array}{cccc}\mathrm{N} & 2.35269 & -0.66089 & -0.55353 \\ \mathrm{C} & 1.53966 & -0.58091 & 2.92676 \\ \mathrm{C} & 1.91349 & -0.43967 & -1.92537 \\ \mathrm{C} & 3.00729 & -0.3346 & -2.94855 \\ \mathrm{O} & 0.73467 & -0.57744 & -2.16847 \\ \mathrm{C} & -5.10772 & 0.6969 & 0.06005 \\ \mathrm{C} & -4.45034 & 2.05782 & 0.32365 \\ \mathrm{C} & -4.12389 & -0.26511 & -0.6189 \\ \mathrm{C} & -3.15965 & 1.88931 & 1.13632 \\ \mathrm{C} & -2.81999 & -0.43296 & 0.18791 \\ \mathrm{C} & -2.16075 & 0.92366 & 0.47962 \\ \mathrm{O} & -1.03375 & 0.73134 & 1.32579 \\ \mathrm{~N} & -1.88849 & -1.32961 & -0.49411 \\ \mathrm{C} & -1.95037 & -2.68494 & -0.33202 \\ \mathrm{O} & -2.71995 & -3.22957 & 0.45444 \\ \mathrm{C} & 1.55215 & 2.47544 & -0.64187 \\ \mathrm{C} & 1.2405 & 3.96924 & -0.76648 \\ \mathrm{O} & 1.12123 & 1.88745 & 0.40415 \\ \mathrm{O} & 2.20787 & 1.92982 & -1.56532 \\ \mathrm{C} & -0.99485 & -3.49317 & -1.20091 \\ \mathrm{H} & 5.57257 & -0.72884 & 1.93046 \\ \mathrm{H} & 4.49209 & -1.60613 & 3.02303 \\ \mathrm{H} & 4.51756 & -0.65141 & -0.65486 \\ \mathrm{H} & 1.96811 & 0.26961 & 3.46072 \\ \mathrm{H} & 0.47295 & -0.4049 & 2.78144 \\ \mathrm{H} & 1.70163 & -1.49695 & 3.50002 \\ \mathrm{H} & 0.40642 & -0.57123 & 0.39799 \\ \mathrm{H} & 3.73729 & 0.42076 & -2.66217 \\ \mathrm{H} & 3.49432 & -1.31038 & -3.06956 \\ \mathrm{H} & 2.54748 & -0.04879 & -3.89345 \\ \mathrm{H} & 1.49922 & 4.48637 & 0.16244 \\ \mathrm{H} & 1.77726 & 4.4143 & -1.60622 \\ \mathrm{H} & 0.16396 & 4.10501 & -0.92068 \\ \mathrm{H} & -0.32624 & 1.36741 & 1.04838 \\ \mathrm{H} & -3.39361 & 1.49502 & 2.13416 \\ \mathrm{H} & -2.66212 & 2.85401 & 1.28972 \\ \mathrm{H} & -5.14185 & 2.72668 & 0.84944 \\ \mathrm{H} & -4.22006 & 2.54125 & -0.6365 \\ \mathrm{H} & -5.44078 & 0.26366 & 1.01336 \\ \mathrm{H} & -6.00472 & 0.8143 & -0.55929 \\ \mathrm{H} & -3.87332 & 0.11261 & -1.62064 \\ \mathrm{H} & -4.57413 & -1.25378 & -0.75376 \\ \mathrm{H} & -3.05659 & -0.91303 & 1.1436 \\ \mathrm{H} & -1.82819 & 1.3563 & -0.48009 \\ \mathrm{H} & -1.27324 & -0.94931 & -1.20419 \\ \mathrm{H} & -0.47192 & -4.21331 & -0.56617 \\ \mathrm{H} & -0.2708 & -2.87987 & -1.7417 \\ \mathrm{H} & -1.58198 & -4.06874 & -1.92327 \\ \mathrm{H} & 4.5211 & 0.16368 & 3.03558\end{array}$

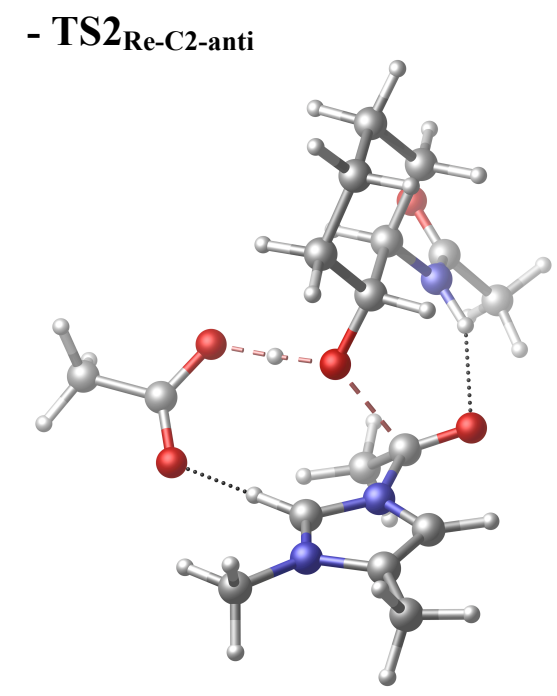

$\begin{array}{llll}\text { C } & -5.03696 & -2.36508 & 0.91632\end{array}$

$\begin{array}{llll}\text { C } & -3.87473 & -1.65518 & 0.30872\end{array}$

$\begin{array}{llll}\mathrm{N} & -3.83178 & -0.26709 & 0.13648\end{array}$

C $\quad-2.68973-2.12443-0.18031$

$\begin{array}{lllll}\text { C } & -2.66827 & 0.07648 & -0.43454\end{array}$

$\begin{array}{llll}\mathrm{N} & -1.9697 & -1.04037 & -0.64263\end{array}$

$\begin{array}{llll}\text { C } & -4.8599 & 0.69455 & 0.52655\end{array}$

C $\quad-0.56796-1.13588$ - 1.19308

$\begin{array}{llll}\text { C } & -0.3452 & -0.25753 & -2.4015\end{array}$

$\begin{array}{llll}\text { O } & -0.02261 & -2.21356 & -1.00115\end{array}$

$\begin{array}{llll}\text { C } & 3.27515 & 0.10776 & 3.0179\end{array}$

$\begin{array}{llll}\text { C } & 1.82349 & -0.01035 & 3.50101\end{array}$

$\begin{array}{llll}\text { C } & 3.47953 & -0.66309 & 1.70827\end{array}$

$\begin{array}{llll}\text { C } & 0.84439 & 0.44909 & 2.41199\end{array}$

$\begin{array}{llll}\text { C } & 2.50812 & -0.19596 & 0.60465\end{array}$

$\begin{array}{llll}\text { C } & 1.04274 & -0.29521 & 1.07941\end{array}$

$\begin{array}{lllll}\mathrm{O} & 0.11828 & 0.19112 & 0.12532\end{array}$

$\begin{array}{llll}\mathrm{N} & 2.6809 & -0.97818 & -0.61779\end{array}$

C $3.75289-0.82058-1.44113$

$\begin{array}{llll}\text { O } & 4.65585 & -0.01551 & -1.22586\end{array}$

$\begin{array}{llll}\text { C } & -0.64955 & 3.23055 & -0.65543\end{array}$

$\begin{array}{llll}\text { C } & -0.41592 & 4.72988 & -0.76602\end{array}$

$\begin{array}{llll}\mathrm{O} & -1.78491 & 2.76537 & -0.90099\end{array}$

$\begin{array}{llll}\text { O } & 0.38842 & 2.5504 & -0.31596\end{array}$

$\begin{array}{llll}\text { C } & 3.7675 & -1.72585 & -2.66845\end{array}$

$\begin{array}{llll}\mathrm{H} & -5.22839 & -2.03052 & 1.94216\end{array}$

H $\quad-4.83522 \quad-3.43736 \quad 0.94819$

$\begin{array}{llll}\mathrm{H} & -2.28341 & -3.12045 & -0.23938\end{array}$

$\begin{array}{llll}\mathrm{H} & -5.05459 & 0.62363 & 1.5992\end{array}$

$\begin{array}{lllll}\mathrm{H} & -4.49618 & 1.69495 & 0.29107\end{array}$

$\begin{array}{llll}\mathrm{H} & -5.78532 & 0.50493 & -0.02278\end{array}$

$\begin{array}{llll}\mathrm{H} & -2.31929 & 1.10656 & -0.63076\end{array}$

$\begin{array}{llll}\mathrm{H} & -0.78613 & -0.75923 & -3.27126\end{array}$

$\mathrm{H} \quad-0.78383 \quad 0.73486 \quad-2.29575$

$\mathrm{H} \quad 0.72883-0.15935 \quad-2.55534$

$\begin{array}{llll}\mathrm{H} & 0.37851 & 4.92437 & -1.49281\end{array}$

$\begin{array}{llll}\mathrm{H} & -1.3295 & 5.24297 & -1.06837\end{array}$ 


$\begin{array}{cccc}\mathrm{H} & -0.06977 & 5.12036 & 0.19591 \\ \mathrm{H} & 0.23773 & 1.32946 & -0.12505 \\ \mathrm{H} & 0.97523 & 1.52228 & 2.22274 \\ \mathrm{H} & -0.19512 & 0.31035 & 2.73164 \\ \mathrm{H} & 1.66905 & 0.57677 & 4.41448 \\ \mathrm{H} & 1.61461 & -1.05744 & 3.76382 \\ \mathrm{H} & 3.52095 & 1.1671 & 2.86076 \\ \mathrm{H} & 3.9665 & -0.26214 & 3.78439 \\ \mathrm{H} & 3.31875 & -1.73671 & 1.88271 \\ \mathrm{H} & 4.50034 & -0.54507 & 1.33502 \\ \mathrm{H} & 2.7287 & 0.85186 & 0.36428 \\ \mathrm{H} & 0.81562 & -1.36441 & 1.22935 \\ \mathrm{H} & 1.96244 & -1.65596 & -0.84788 \\ \mathrm{H} & 2.86674 & -2.33678 & -2.77438 \\ \mathrm{H} & 4.63907 & -2.38461 & -2.6112 \\ \mathrm{H} & 3.89029 & -1.10447 & -3.55959 \\ \mathrm{H} & -5.95638 & -2.21406 & 0.33934\end{array}$

\section{- TS2 $2_{\text {Re-C2-syn }}$}

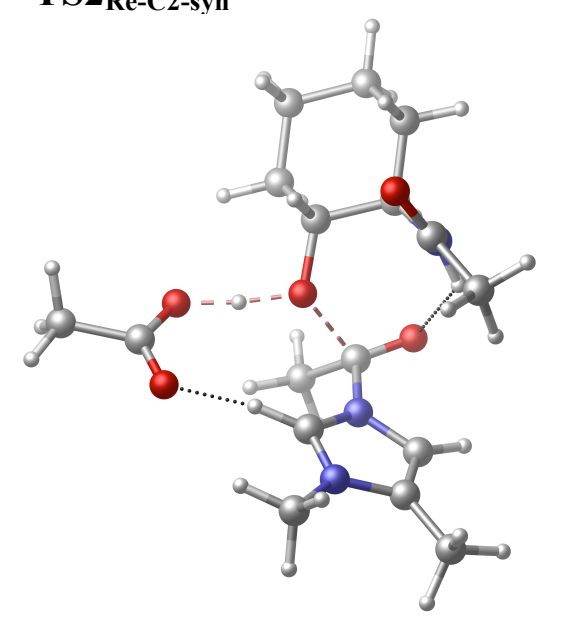

$\begin{array}{llll}\text { C } & 0.36858 & 3.49865 & -0.79902\end{array}$

$\begin{array}{llll}\text { C } & 0.03166 & 4.91424 & -1.23453\end{array}$

$\begin{array}{llll}\mathrm{O} & 1.43416 & 2.96718 & -1.15275\end{array}$

$\begin{array}{lllll}\mathrm{O} & -0.53655 & 2.94464 & -0.05138\end{array}$

$\begin{array}{llll}\text { C } & 0.45041 & -3.20842 & -1.82663\end{array}$

$\begin{array}{llll}\mathrm{H} & 4.80599 & -2.45387 & -0.76236\end{array}$

$\begin{array}{llll}\mathrm{H} & 4.68342 & -2.95586 & 0.92775\end{array}$

$\begin{array}{llll}\mathrm{H} & 2.20674 & -2.12513 & 2.02047\end{array}$

$\begin{array}{llll}\mathrm{H} & 4.26554 & -0.15752 & -2.10928\end{array}$

$\begin{array}{llll}\mathrm{H} & 3.69708 & 1.45839 & -1.61765\end{array}$

$\begin{array}{llll}\mathrm{H} & 5.15815 & 0.76086 & -0.86877\end{array}$

$\begin{array}{llll}\mathrm{H} & 1.74863 & 1.42846 & -0.26453\end{array}$

$\begin{array}{llll}\mathrm{H} & 0.99677 & 1.01664 & 3.54201\end{array}$

$\begin{array}{llll}\mathrm{H} & 0.56287 & 2.03692 & 2.14125\end{array}$

$\begin{array}{llll}\mathrm{H} & -0.70842 & 1.28133 & 3.1245\end{array}$

$\begin{array}{llll}\mathrm{H} & -0.23672 & 5.51888 & -0.36357\end{array}$

$\begin{array}{llll}\mathrm{H} & 0.87351 & 5.3645 & -1.76119\end{array}$

$\begin{array}{lllll}\mathrm{H} & -0.84307 & 4.89283 & -1.89237\end{array}$

$\begin{array}{llll}\mathrm{H} & -0.49853 & 1.76741 & 0.15129\end{array}$

$\begin{array}{llll}\mathrm{H} & -2.97895 & 0.82033 & 1.74134\end{array}$

$\begin{array}{llll}\mathrm{H} & -2.81367 & 2.06874 & 0.50447\end{array}$

$\begin{array}{llll}\mathrm{H} & -5.18513 & 1.23331 & 0.65531\end{array}$

$\begin{array}{llll}\mathrm{H} & -4.48817 & 0.92923 & -0.93036\end{array}$

H $\quad-4.77742 \quad-1.1104 \quad 1.33726$

H $\quad-5.67815 \quad-1.10312-0.17434$

$\begin{array}{llll}\mathrm{H} & -3.58899 & -1.54193 & -1.46651\end{array}$

$\begin{array}{llll}\mathrm{H} & -3.77889 & -2.76541 & -0.21475\end{array}$

$\begin{array}{llll}\mathrm{H} & -2.25331 & -1.50294 & 1.27242\end{array}$

$\begin{array}{llll}\mathrm{H} & -1.97895 & 0.35005 & -1.11505\end{array}$

$\begin{array}{lllll}\mathrm{H} & -0.47805 & -2.44378 & 0.42868\end{array}$

$\begin{array}{llll}\mathrm{H} & 1.077 & -3.33248 & -0.93843\end{array}$

$\begin{array}{llll}\mathrm{H} & 1.03947 & -2.76432 & -2.63183\end{array}$

$\begin{array}{llll}\mathrm{H} & 0.12754 & -4.19907 & -2.16283\end{array}$

$\begin{array}{llll}\mathrm{H} & 5.66667 & -1.55262 & 0.49369\end{array}$

\section{- TS2 $2_{\text {Re-C4-anti }}$}

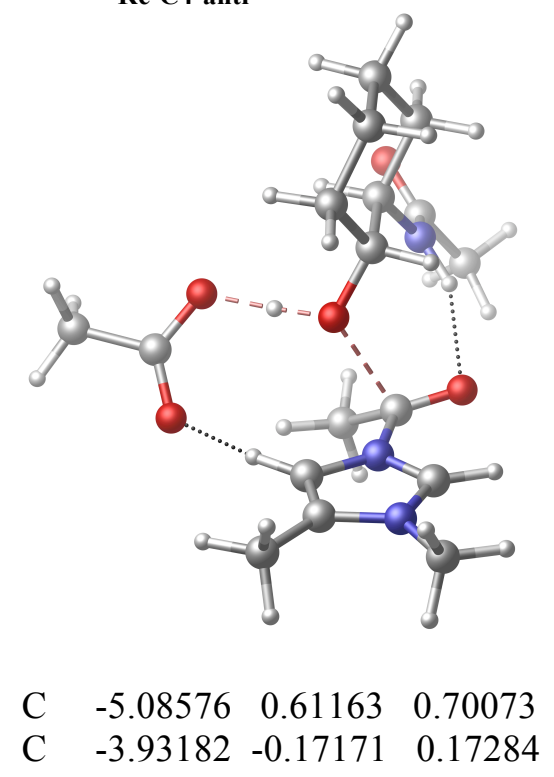



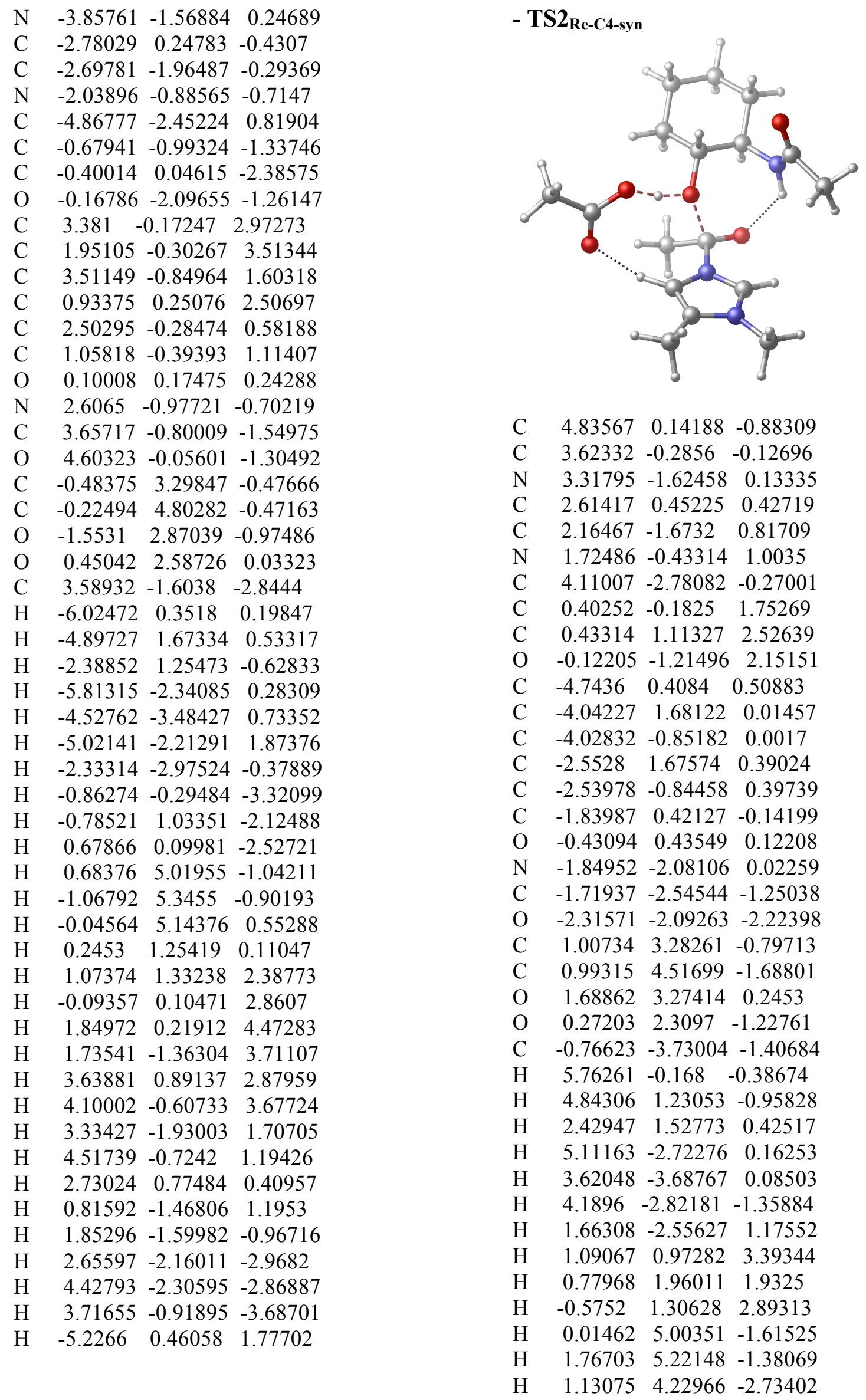

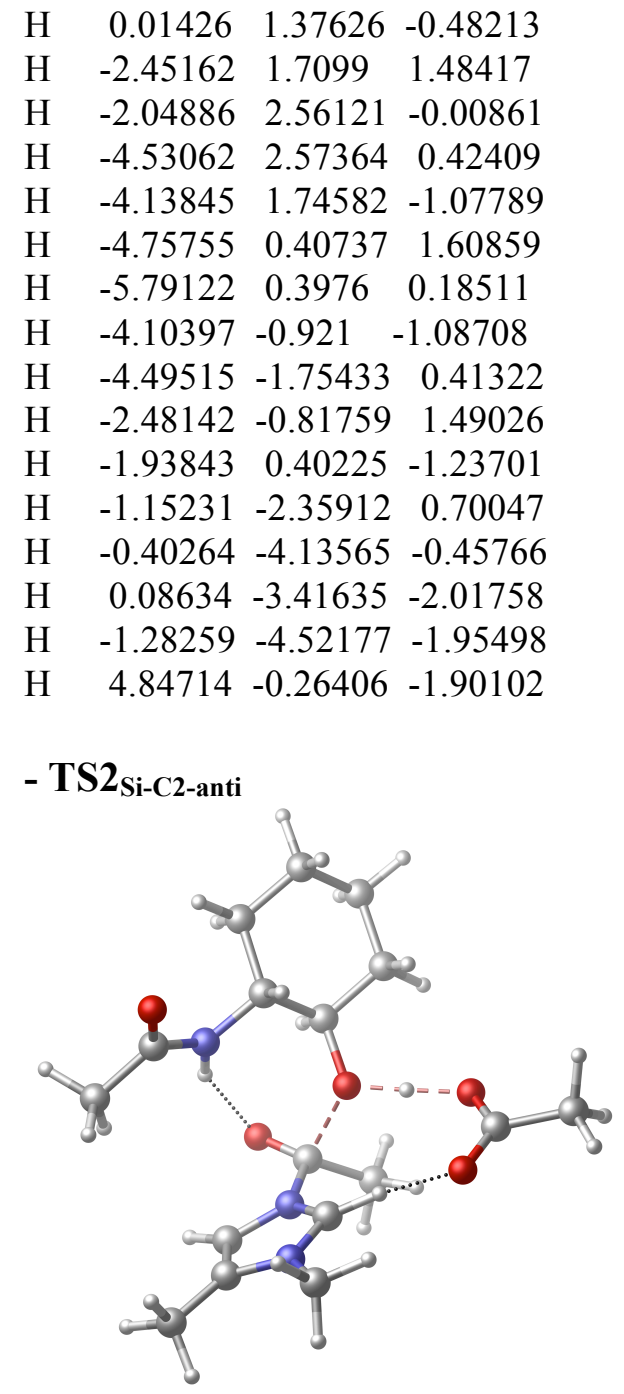
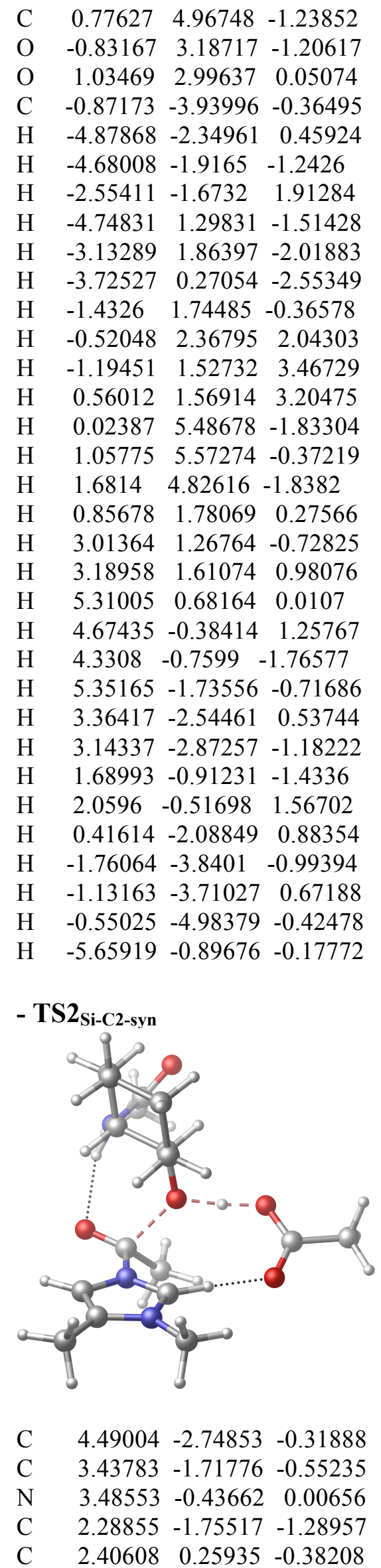


$\begin{array}{cccc}\mathrm{N} & 1.67513 & -0.52403 & -1.17376 \\ \mathrm{C} & 4.51196 & 0.08952 & 0.9028 \\ \mathrm{C} & 0.33751 & -0.1889 & -1.82135 \\ \mathrm{C} & 0.34827 & 1.16267 & -2.50256 \\ \mathrm{O} & -0.25903 & -1.16284 & -2.25649 \\ \mathrm{C} & -1.8963 & -2.5851 & 2.43009 \\ \mathrm{C} & -1.0438 & -1.45435 & 3.02369 \\ \mathrm{C} & -2.81366 & -2.06483 & 1.31465 \\ \mathrm{C} & -0.25264 & -0.72891 & 1.92558 \\ \mathrm{C} & -2.00575 & -1.34829 & 0.21663 \\ \mathrm{C} & -1.16999 & -0.19084 & 0.81279 \\ \mathrm{O} & -0.43272 & 0.52232 & -0.1723 \\ \mathrm{~N} & -2.83366 & -0.91746 & -0.91094 \\ \mathrm{C} & -3.87293 & -0.03978 & -0.8128 \\ \mathrm{O} & -4.35975 & 0.33736 & 0.25074 \\ \mathrm{C} & 0.69299 & 3.43235 & 0.70149 \\ \mathrm{C} & 0.55391 & 4.84155 & 1.25171 \\ \mathrm{O} & 1.82285 & 2.93325 & 0.53988 \\ \mathrm{O} & -0.43473 & 2.85899 & 0.43155 \\ \mathrm{C} & -4.4259 & 0.45291 & -2.14645 \\ \mathrm{H} & 4.23287 & -3.66259 & -0.85728 \\ \mathrm{H} & 4.58505 & -3.00186 & 0.74307 \\ \mathrm{H} & 1.84047 & -2.53803 & -1.87876 \\ \mathrm{H} & 5.48305 & 0.10244 & 0.40193 \\ \mathrm{H} & 4.23275 & 1.1063 & 1.17987 \\ \mathrm{H} & 4.57741 & -0.52562 & 1.80336 \\ \mathrm{H} & 2.14648 & 1.27714 & -0.05254 \\ \mathrm{H} & 0.82459 & 1.94281 & -1.90964 \\ \mathrm{H} & 0.8808 & 1.05357 & -3.45432 \\ \mathrm{H} & -0.68362 & 1.44748 & -2.70595 \\ \mathrm{H} & 1.53465 & 5.29021 & 1.41198 \\ \mathrm{H} & -0.0296 & 5.45308 & 0.55732 \\ \mathrm{H} & -0.00067 & 4.81339 & 2.19477 \\ \mathrm{H} & -0.38568 & 1.7337 & 0.10765 \\ \mathrm{H} & 0.47393 & -1.42371 & 1.47807 \\ \mathrm{H} & 0.31983 & 0.10789 & 2.3433 \\ \mathrm{H} & -0.36267 & -1.84593 & 3.78958 \\ \mathrm{H} & -1.70166 & -0.73444 & 3.52936 \\ \mathrm{H} & -1.23166 & -3.36475 & 2.0289 \\ \mathrm{H} & -2.49612 & -3.06257 & 3.21412 \\ \mathrm{H} & -3.55125 & -1.36773 & 1.72163 \\ \mathrm{H} & -3.37222 & -2.89028 & 0.858 \\ \mathrm{H} & -1.30643 & -2.07274 & -0.21406 \\ \mathrm{H} & -1.88121 & 0.52007 & 1.2592 \\ \mathrm{H} & -2.36502 & -0.99432 & -1.80462 \\ \mathrm{H} & -5.51523 & 0.37366 & -2.12283 \\ \mathrm{H} & -4.04285 & -0.09438 & -3.01216 \\ \mathrm{H} & -4.17832 & 1.51329 & -2.25875 \\ \mathrm{H} & 5.47335 & -2.41718 & -0.67167 \\ & & & \end{array}$
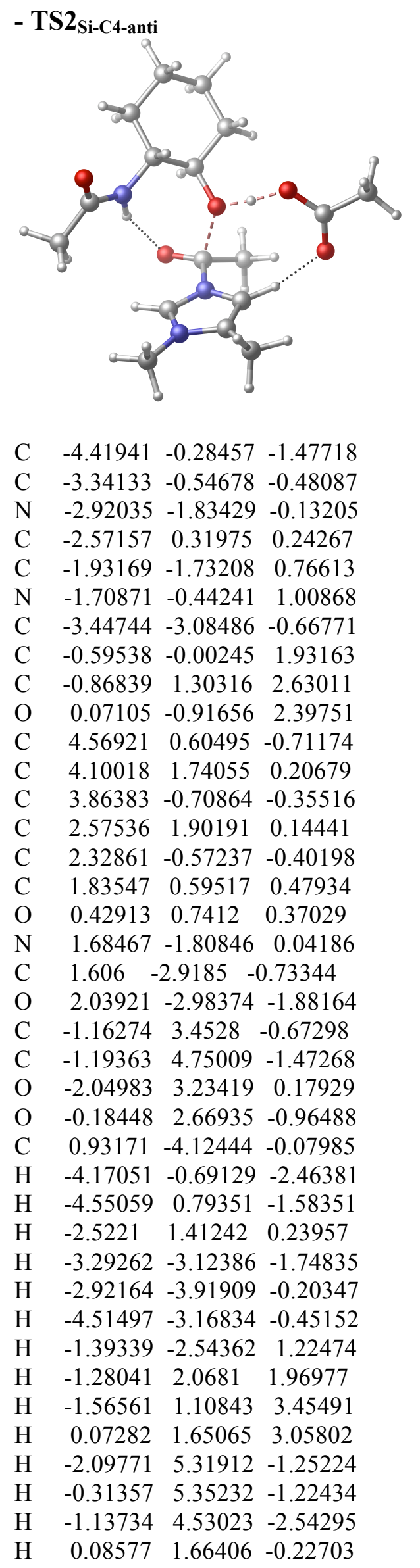

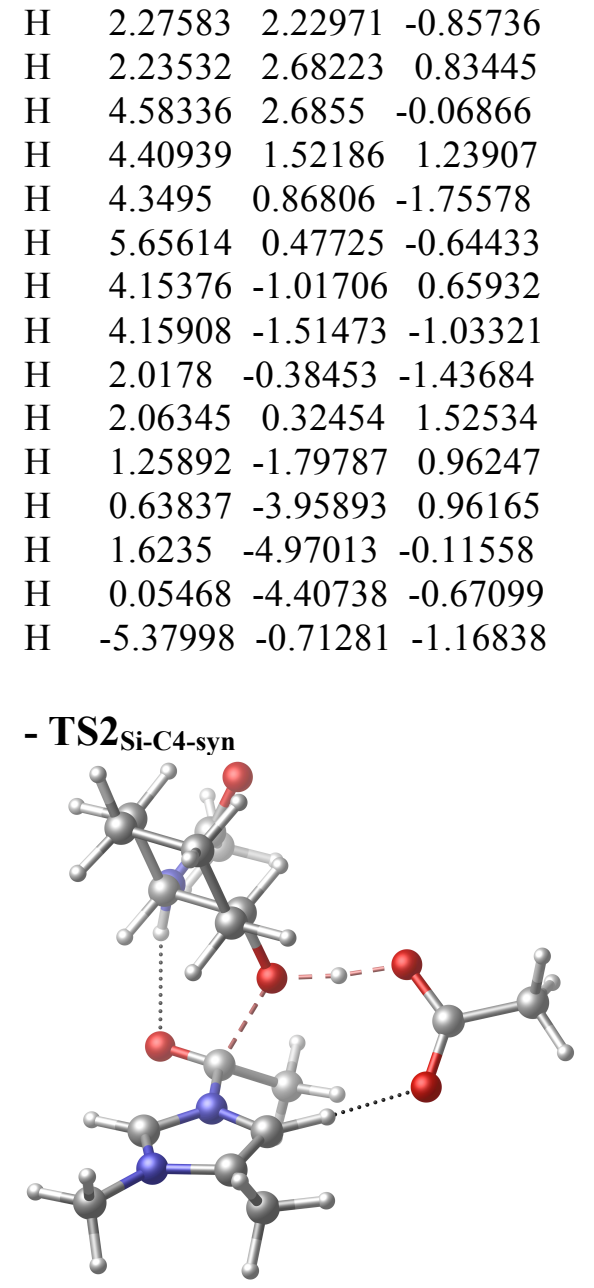

$\begin{array}{llll}\mathrm{C} & 4.73062 & -0.27688 & 0.9008 \\ \mathrm{C} & 3.54479 & -0.52383 & 0.03083 \\ \mathrm{~N} & 3.27325 & -1.76463 & -0.55813 \\ \mathrm{C} & 2.53333 & 0.31393 & -0.34754 \\ \mathrm{C} & 2.13743 & -1.66164 & -1.2642 \\ \mathrm{~N} & 1.67834 & -0.4179 & -1.14966 \\ \mathrm{C} & 4.0803 & -2.97261 & -0.42855 \\ \mathrm{C} & 0.38896 & 0.00494 & -1.83256 \\ \mathrm{C} & 0.43889 & 1.41111 & -2.37288 \\ \mathrm{O} & -0.22337 & -0.91815 & -2.34557 \\ \mathrm{C} & -1.83445 & -2.66291 & 2.33474 \\ \mathrm{C} & -1.06426 & -1.51363 & 3.00061 \\ \mathrm{C} & -2.75197 & -2.14449 & 1.21873 \\ \mathrm{C} & -0.28256 & -0.70305 & 1.9576 \\ \mathrm{C} & -1.96075 & -1.33648 & 0.17298 \\ \mathrm{C} & -1.19146 & -0.17073 & 0.8359 \\ \mathrm{O} & -0.45496 & 0.59413 & -0.10842 \\ \mathrm{~N} & -2.79021 & -0.89106 & -0.94879 \\ \mathrm{C} & -3.88263 & -0.0818 & -0.82686 \\ \mathrm{O} & -4.41999 & 0.19784 & 0.24201 \\ \mathrm{C} & 0.71355 & 3.50919 & 0.74327 \\ \mathrm{C} & 0.62089 & 4.87991 & 1.4003 \\ \mathrm{O} & 1.79524 & 3.13084 & 0.25107\end{array}$

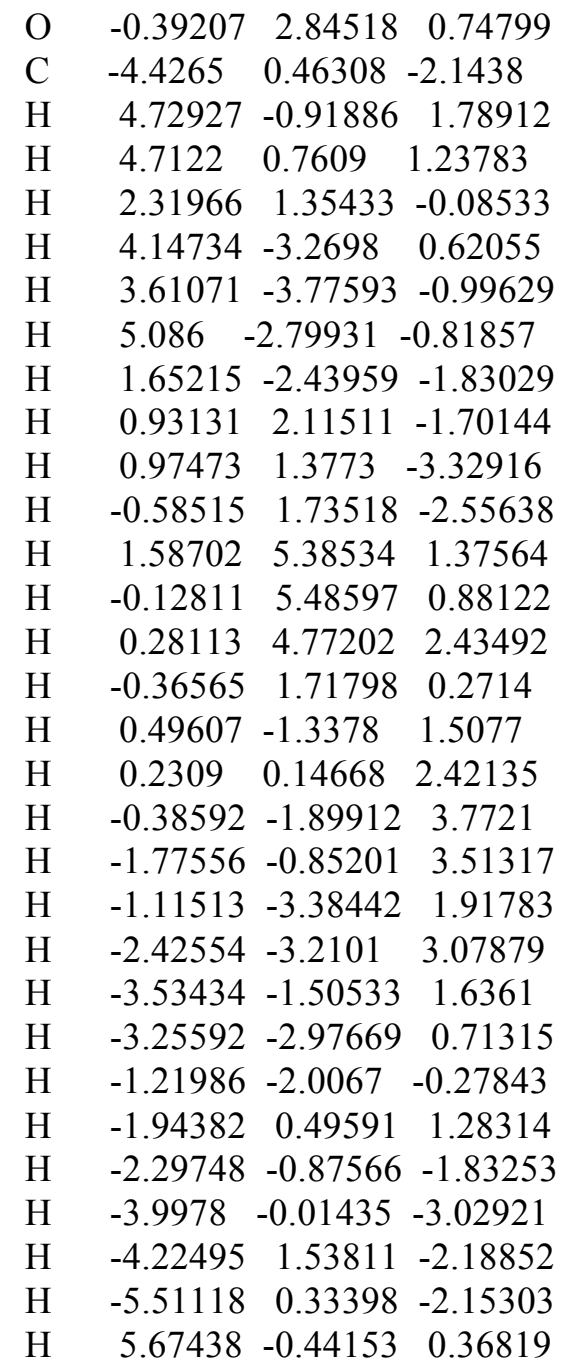

\section{- $(R, R)-\mathrm{A} 1 \mathrm{~B} 1 \mathrm{C} 1 S i$}

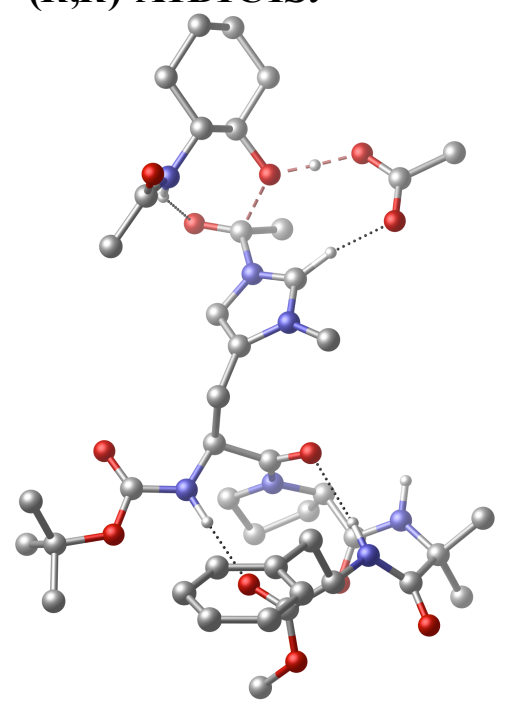

$\begin{array}{lrrr}\mathrm{N} & -1.93249 & 1.62489 & -1.10417 \\ \mathrm{C} & -0.74966 & 0.78585 & -1.15968 \\ \mathrm{C} & 0.23604 & 1.09283 & -0.00308\end{array}$ 
$\mathrm{H} \quad-5.34607 \quad 4.55042 \quad-1.84405$

$\mathrm{H} \quad-4.96136 \quad 6.24562-1.48125$

$\mathrm{H} \quad-4.84414 \quad 5.01316-0.20828$

$\mathrm{H} \quad-3.66685 \quad 4.49101 \quad-3.7237$

$\begin{array}{llll}\mathrm{H} & -3.2715 & 6.1927 & -3.43242\end{array}$

$\mathrm{H} \quad-1.97856 \quad 4.975 \quad-3.44045$

$\mathrm{H} \quad-2.50639 \quad 6.90035-1.0072$

$\mathrm{H} \quad-1.24346 \quad 5.65317-1.06471$

$\mathrm{H} \quad-2.44094 \quad 5.65472 \quad 0.25036$

$\begin{array}{lllll}\mathrm{H} & -2.80337 & 1.27184 & -0.71073\end{array}$

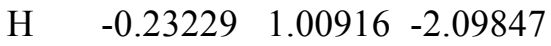

$\mathrm{H} \quad \begin{array}{llll}0.3965 & 2.17403 & -0.01764\end{array}$

$\begin{array}{llll}\mathrm{H} & -0.23512 & 0.833 & 0.94632\end{array}$

$\mathrm{H} \quad 2.6184 \quad 1.23382 \quad-1.91693$

$\mathrm{H} \quad 0.35063 \quad-1.45994 \quad 1.6576$

H $\quad 1.9562 \quad-1.95223 \quad 2.29115$

$\begin{array}{llll}\mathrm{H} & 1.31027 & -0.33754 & 2.66918\end{array}$

$\begin{array}{llll}\mathrm{H} & 3.79847 & -1.82157 & 0.79668\end{array}$

$\mathrm{H} \quad 4.9203 \quad-2.62058-1.45134$

$\mathrm{H} \quad 4.32946-1.98325 \quad-3.01181$

$\mathrm{H} \quad \begin{array}{llll}6.05125 & -1.8706 & -2.59725\end{array}$

$\begin{array}{llll}\mathrm{H} & 5.42685 & -5.39847 & 2.54427\end{array}$

$\mathrm{H} \quad \begin{array}{llll}6.64006 & -5.44773 & 1.22576\end{array}$

H $\quad \begin{array}{llll}7.00155 & -4.57819 & 2.71213\end{array}$

$\begin{array}{llll}\mathrm{H} & 6.15307 & -1.7457 & 0.30151\end{array}$

$\mathrm{H} \quad 8.16282 \quad-0.93008 \quad 1.42057$

H $\quad 8.50064 \quad-1.43957-0.22092$

$\mathrm{H} \quad \begin{array}{llll}10.45726 & -0.22895 & 0.78255\end{array}$

$\begin{array}{lllll}\mathrm{H} & 9.84086 & 0.63251 & -0.62204\end{array}$

$\begin{array}{llll}\mathrm{H} & 9.23184 & 1.31173 & 2.30522\end{array}$

$\begin{array}{llll}\mathrm{H} & 10.24966 & 2.25057 & 1.22034\end{array}$

H $\quad 8.30612 \quad 2.73562 \quad-0.25201$

$\begin{array}{llll}\mathrm{H} & 7.92792 & 3.23061 & 1.39895\end{array}$

$\begin{array}{llll}\mathrm{H} & 6.62602 & 1.1859 & 1.77977\end{array}$

$\mathrm{H} \quad \begin{array}{llll}7.25482 & 0.50491 & -1.12534\end{array}$

$\mathrm{H} \quad 5.43651 \quad 2.00066-0.7314$

$\begin{array}{llll}\mathrm{H} & 2.99062 & 3.72339 & 0.82171\end{array}$

H $\quad 3.73762 \quad 3.48495 \quad-0.78108$

$\mathrm{H} \quad 4.13347 \quad 4.91054 \quad 0.20533$

$\mathrm{H} \quad-2.29666 \quad 0.40623 \quad-3.44022$

H $\quad-0.79949 \quad-0.31051 \quad-4.07973$

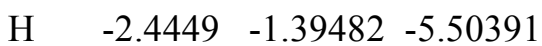

$\mathrm{H} \quad-3.62936-1.46637 \quad-4.17717$

$\mathrm{H} \quad-2.71835 \quad-3.72484 \quad-4.14272$

$\mathrm{H} \quad-1.07318-3.13035 \quad-4.41991$

$\mathrm{H} \quad-0.92491 \quad-3.22298 \quad-2.06755$

H $\quad-1.79538 \quad-4.02826 \quad-0.30223$

$\mathrm{H} \quad-2.2932 \quad-4.61298 \quad 1.98553$

H $\quad-2.45703 \quad-6.1042 \quad 1.02353$ 


$\begin{array}{llcc}\mathrm{H} & -3.712 & -5.65187 & 2.19335 \\ \mathrm{H} & -4.20031 & -6.27223 & -0.77843 \\ \mathrm{H} & -5.50157 & -5.77671 & 0.33764 \\ \mathrm{H} & -5.24852 & -4.88677 & -1.17062 \\ \mathrm{H} & -3.02911 & -2.01447 & 0.75539 \\ \mathrm{H} & -5.48803 & -1.32551 & 2.26191 \\ \mathrm{H} & -6.33363 & -0.57044 & -1.91238 \\ \mathrm{H} & -7.93913 & -0.3769 & -1.13285 \\ \mathrm{H} & -6.78637 & 0.99917 & -1.18974 \\ \mathrm{H} & -3.31641 & -0.69319 & 3.35383 \\ \mathrm{H} & -2.8721 & 0.24869 & 1.93072 \\ \mathrm{H} & -5.28662 & 0.03066 & 4.76869 \\ \mathrm{H} & -6.49963 & 1.96992 & 5.70735 \\ \mathrm{H} & -6.34414 & 4.19276 & 4.60165 \\ \mathrm{H} & -4.96695 & 4.45099 & 2.54886 \\ \mathrm{H} & -3.77159 & 2.50792 & 1.59922\end{array}$

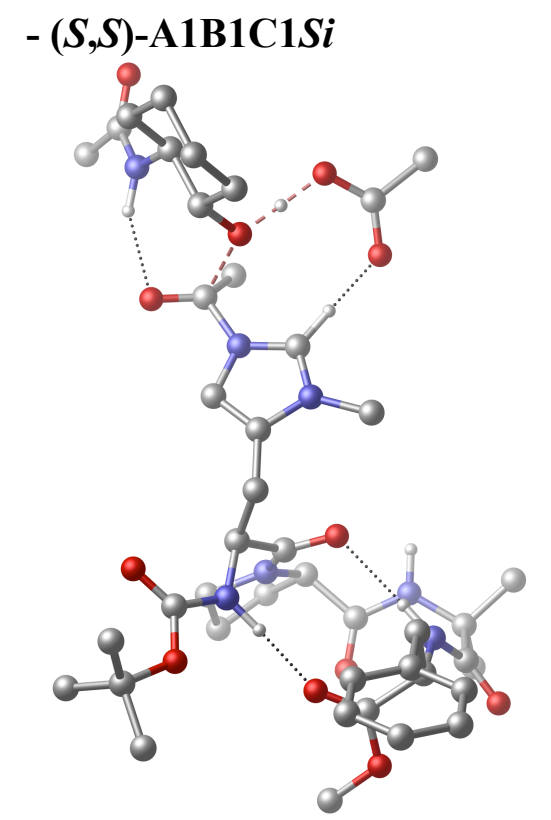

$\begin{array}{lccc}\mathrm{N} & -2.13719 & 1.74716 & -1.01757 \\ \mathrm{C} & -0.94579 & 0.91879 & -1.03224 \\ \mathrm{C} & -0.04476 & 1.17735 & 0.20256 \\ \mathrm{C} & -1.35771 & -0.56169 & -1.1241 \\ \mathrm{C} & 1.28145 & 0.48447 & 0.12909 \\ \mathrm{O} & -1.39489 & -1.28151 & -0.1111 \\ \mathrm{~N} & 1.67683 & -0.55697 & 0.97602 \\ \mathrm{C} & 2.32721 & 0.67549 & -0.73208 \\ \mathrm{C} & 2.90218 & -0.97214 & 0.63119 \\ \mathrm{~N} & 3.30592 & -0.2435 & -0.41274 \\ \mathrm{~N} & -1.65383 & -1.0511 & -2.34499 \\ \mathrm{C} & -1.77075 & -0.2866 & -3.6031 \\ \mathrm{C} & -1.89631 & -2.49496 & -2.53211 \\ \mathrm{C} & -2.51682 & -1.25761 & -4.52398 \\ \mathrm{C} & -2.01201 & -2.63495 & -4.06575 \\ \mathrm{C} & 0.9292 & -1.10806 & 2.10783 \\ \mathrm{C} & -4.04961 & -4.44677 & 0.00794\end{array}$

\begin{tabular}{|c|c|c|c|}
\hline & & & \\
\hline & -4.87266 & -3.35387 & \\
\hline & & -3.58482 & \\
\hline & 36733 & -5.31357 & \\
\hline & & -5.29939 & \\
\hline & .03869 & & \\
\hline & .00073 & & \\
\hline & .23601 & & \\
\hline & .41841 & 5.10 & \\
\hline & 572 & & \\
\hline & -4.89948 & & \\
\hline & -2.53247 & & \\
\hline & 463 & & \\
\hline & 75 & 2.2 & \\
\hline & -6.23 & 3.15 & 4.20023 \\
\hline & 2 & 1.00 & \\
\hline & 93 & & \\
\hline & 58 & -0.1 & \\
\hline & 6 & & \\
\hline & 3 & -1.0 & \\
\hline & -4.2 & -2.1 & \\
\hline & -5.3 & -0.2 & \\
\hline & -4.6 & & \\
\hline & 62 & -0. & \\
\hline & -7.0 & & \\
\hline & -4.2 & & \\
\hline & -3.1 & -2.9 & \\
\hline & 6.32 & & \\
\hline & & & \\
\hline & & 1.0 & \\
\hline & & & \\
\hline & 7. & 0.92 & \\
\hline & 35 & & \\
\hline & & -0 . & \\
\hline & 65 & & \\
\hline & 42 & & \\
\hline & 23 & & \\
\hline & & & \\
\hline & & & \\
\hline & & & \\
\hline & 71 & & \\
\hline & & & \\
\hline & & & \\
\hline & & & \\
\hline & & -3.9 & 3.218 \\
\hline & & & \\
\hline & & & \\
\hline & & & \\
\hline & & & \\
\hline & -3.38512 & & -3.22569 \\
\hline & -2.08539 & 5.18408 & -3.21176 \\
\hline & & 5.72166 & \\
\hline $\mathrm{H}$ & & & \\
\hline & -2.75393 & 7.01607 & $=7370$ \\
\hline
\end{tabular}




\begin{tabular}{|c|c|c|}
\hline-3.02568 & & \\
\hline-0.36806 & 1.18974 & \\
\hline 0.11374 & 2.2583 & 0.24212 \\
\hline-0.58366 & 0.88142 & 1.10427 \\
\hline 0.79804 & -0.3422 & 2.87631 \\
\hline 1.50968 & -1.93312 & 2.52136 \\
\hline-0.04247 & -1.45674 & 1.75817 \\
\hline 3.49999 & -1.74051 & 1.15005 \\
\hline 2.47692 & 1.38443 & -1.52983 \\
\hline 6.15611 & -1.71651 & -1.6036 \\
\hline 4.54733 & -2.09278 & -2.26375 \\
\hline 4.90901 & -2.4 & -0.54489 \\
\hline 6.63796 & -3.51526 & 1846 \\
\hline 7.07921 & -4.48697 & 2.72123 \\
\hline 5.48 & -4.7 & 3.49351 \\
\hline 6.03 & -1.0 & 1.22249 \\
\hline 5.86341 & 1.8 & 0.2 \\
\hline 5.27339 & 1.50055 & 2.6 \\
\hline 6.73502 & 0.55935 & 2.9 \\
\hline 7.08589 & 2.88353 & 3.7 \\
\hline 6.62655 & 3.53482 & 2.1 \\
\hline 9.10239 & 3.4996 & \\
\hline 9.07844 & 1.78355 & 2.7 \\
\hline 9.62698 & 2.09041 & 0.2 \\
\hline 8.1 & 3.04081 & 0.0 \\
\hline 8.3 & 0.08 & \\
\hline 6.95596 & 0.776 & -1.6 \\
\hline 7.7084 & 0.0 & -3.5 \\
\hline 9.0 & -1.0 & -3 \\
\hline 9.39 & 0.5 & -3.8 \\
\hline-2.3 & 0.6 & -3. \\
\hline 395 & -0.0 & $5-3.9$ \\
\hline & -1.0 & -5.5 \\
\hline & -1.1 & \\
\hline-2.6 & -3.4 & -4.3 \\
\hline 231 & -2.8 & $5-4.2$ \\
\hline-1.0 & -3.0 & -2.1 \\
\hline-2.0 & -3.9 & $7-0.5$ \\
\hline-4.4 & -6.1 & $5-1.2$ \\
\hline$-5.7 \mathrm{~s}$ & -5.6 & $7-0.22043$ \\
\hline-5.44403 & -4.69 & $\begin{array}{lll}3 & -1.63861\end{array}$ \\
\hline-4.12656 & -5.73902 & $2 \quad 1.7421$ \\
\hline-2.81241 & -6.13486 & 0.61764 \\
\hline-2.6784 & -4.72197 & 1.69544 \\
\hline-3.31194 & -2.00811 & 0.59577 \\
\hline-5.83651 & -1.39208 & 2.02281 \\
\hline-3.20445 & 0.18324 & 1.8935 \\
\hline-3.71012 & -0.83586 & $6 \quad 3.24138$ \\
\hline-6.47115 & -0.40146 & $6-2.14421$ \\
\hline-6.9817 & 1.1158 & -1.35185 \\
\hline-8.11766 & -0.27247 & $7-1.4401$ \\
\hline-5.69534 & -0.17399 & 94.64343 \\
\hline-6.94116 & 1.71425 & 5.6408 \\
\hline-0.114 & & 4.04 \\
\hline
\end{tabular}

$\begin{array}{llll}\mathrm{H} & -5.35244 & 4.34422 & 2.63408 \\ \mathrm{H} & -4.12294 & 2.45259 & 1.62593\end{array}$

\section{- $(R, R)-\mathrm{A} 2 \mathrm{~B} 1 \mathrm{C} 1 S i$}

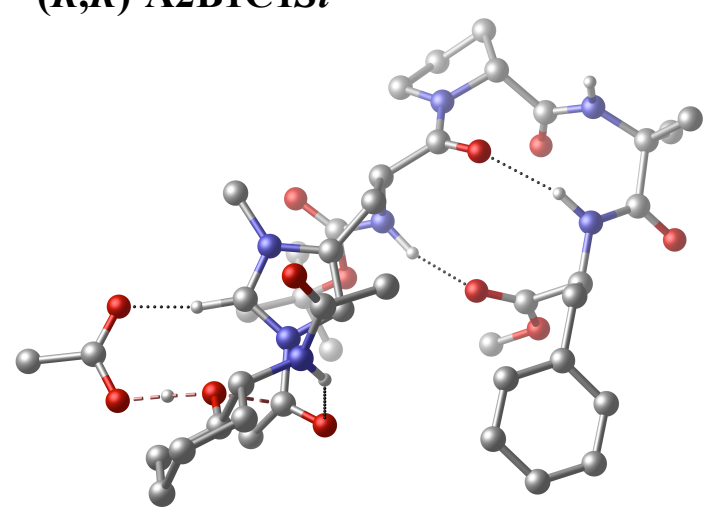

$\begin{array}{llll}\mathrm{N} & -1.49095 & 1.90783 & 0.0702\end{array}$

C $\quad-1.43822 \quad 1.66307-1.36062$

$\begin{array}{llll}\text { C } & -0.3528 & 0.6295 & -1.75602\end{array}$

$\begin{array}{llll}\text { C } & -2.81761 & 1.14363 & -1.83015\end{array}$

$\begin{array}{llll}\text { C } & 0.95159 & 0.75326 & -1.03979\end{array}$

$\begin{array}{lllll}\mathrm{O} & -3.02266 & -0.06544 & -2.00646\end{array}$

$\begin{array}{lllll}\mathrm{N} & 1.90418 & 1.75686 & -1.24033\end{array}$

$\begin{array}{llll}\text { C } & 1.48735 & -0.07739 & -0.09804\end{array}$

$\begin{array}{llll}\text { C } & 2.96521 & 1.52781 & -0.45479\end{array}$

$\begin{array}{llll}\mathrm{N} & 2.72779 & 0.41801 & 0.25132\end{array}$

$\begin{array}{llll}\mathrm{N} & -3.7979 & 2.05308 & -2.02326\end{array}$

$\begin{array}{llll}\text { C } & -3.7489 & 3.50979 & -1.79679\end{array}$

$\begin{array}{llll}\text { C } & -5.14096 & 1.58364 & -2.39797\end{array}$

$\begin{array}{llll}\text { C } & -5.22353 & 3.87004 & -1.58908\end{array}$

$\begin{array}{llll}\text { C } & -5.95561 & 2.89194 & -2.52194\end{array}$

$\begin{array}{llll}\text { C } & 1.80745 & 2.90992 & -2.13877\end{array}$

$\begin{array}{llll}\text { C } & -6.79156 & -1.58349 & -0.92525\end{array}$

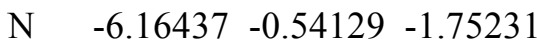

$\begin{array}{llll}\text { C } & -5.82978 & -2.17656 & 0.14425\end{array}$

$\begin{array}{lllll}\text { O } & -6.28963 & -2.83724 & 1.06847\end{array}$

$\begin{array}{llll}\text { C } & -7.16296 & -2.74572 & -1.86717\end{array}$

$\begin{array}{llll}\text { C } & -8.05115 & -1.04563 & -0.2268\end{array}$

$\begin{array}{llll}\text { C } & -0.99187 & 3.05956 & 0.60722\end{array}$

$\begin{array}{llll}\text { O } & -0.64261 & 4.034 & -0.05391\end{array}$

$\begin{array}{llll}\mathrm{O} & -0.97479 & 2.96507 & 1.95402\end{array}$

$\begin{array}{llll}\text { C } & -0.48605 & 4.07823 & 2.79367\end{array}$

$\begin{array}{llll}\text { C } & -1.37405 & 5.31026 & 2.59742\end{array}$

$\begin{array}{llll}\text { C } & -0.64084 & 3.51527 & 4.20808\end{array}$

$\begin{array}{llll}\text { C } & 0.98581 & 4.36148 & 2.47972\end{array}$

$\begin{array}{llll}\text { C } & 0.80522 & -4.14436 & 1.94007\end{array}$

$\begin{array}{llll}\text { C } & -0.15401 & -3.65597 & 1.05177\end{array}$

$\begin{array}{llll}\text { C } & 0.44221 & -5.09832 & 2.89193\end{array}$

$\begin{array}{llll}\text { C } & -1.4823 & -4.09666 & 1.09882\end{array}$

$\begin{array}{llll}\text { C } & -0.87598 & -5.55705 & 2.9448\end{array}$

$\begin{array}{llll}\text { C } & -2.52187 & -3.51985 & 0.15937\end{array}$

$\begin{array}{llll}\text { C } & -1.83029 & -5.05854 & 2.05743\end{array}$

$\begin{array}{llll}\text { C } & -3.51762 & -2.57564 & 0.86569\end{array}$ 


\begin{tabular}{|c|c|c|}
\hline & & \\
\hline & $-2.80073-1.41128$ & \\
\hline & $\begin{array}{lll}-1.90651 & -0.76122\end{array}$ & \\
\hline & $-3.29166-1.16132$ & \\
\hline & $-2.72327-0.03928$ & \\
\hline & $\begin{array}{lll}-5.77766 & 1.03077\end{array}$ & -0.13287 \\
\hline & $-5.7134 \quad 0.66$ & \\
\hline & $16-0.29$ & \\
\hline & $4.06647 \quad 0.5$ & \\
\hline & $3.45136-1.2$ & \\
\hline & $79-2.7$ & \\
\hline & & \\
\hline & $24-3 .($ & \\
\hline & $2-0.8$ & \\
\hline & $07-1$. & \\
\hline & & \\
\hline & & \\
\hline & $9-2$. & \\
\hline & & \\
\hline & $21-2$ & \\
\hline & $2 \quad 3.13$ & \\
\hline & & \\
\hline & & \\
\hline & & \\
\hline & $1-2.8-r-2-x$ & \\
\hline & & \\
\hline & 4.2 & \\
\hline & -0 & \\
\hline & $38 \quad 5.0$ & \\
\hline & 96.0 & \\
\hline & 795 . $^{\prime}$ & \\
\hline & 65.0 & \\
\hline & 384.7 & \\
\hline & & \\
\hline & -1 & \\
\hline & & \\
\hline & $56-0$. & \\
\hline & & \\
\hline & $5-0$. & \\
\hline & 83. & \\
\hline & 83. & \\
\hline & $\begin{array}{ll}6 & 2.5\end{array}$ & \\
\hline & 29 & \\
\hline & 09 & \\
\hline & 0.70 & \\
\hline & 0.0 & \\
\hline & 59 & -0.29 \\
\hline & 4.33 & \\
\hline & 3.88239 & \\
\hline & & \\
\hline & $\begin{array}{ll}7.79121 & -0.05831\end{array}$ & -0.66596 \\
\hline & $\begin{array}{ll}7.71632 & -0.39697\end{array}$ & 1.0509 \\
\hline & $\begin{array}{lll}9.46876 & -1.79311\end{array}$ & -0.08176 \\
\hline & $.2211-2$. & \\
\hline
\end{tabular}

$\begin{array}{llll}\mathrm{H} & 8.41244 & -2.00712 & -2.32562 \\ \mathrm{H} & 8.72797 & -3.60943 & -1.6721 \\ \mathrm{H} & 6.36524 & -3.81966 & -0.93055 \\ \mathrm{H} & 6.40625 & -3.44937 & -2.65536 \\ \mathrm{H} & 5.91517 & -1.08388 & -2.22186 \\ \mathrm{H} & 5.75137 & -1.8703 & 0.71923 \\ \mathrm{H} & 3.81515 & -2.25932 & -0.61565 \\ \mathrm{H} & 1.59263 & -2.0411 & -2.95761 \\ \mathrm{H} & 1.90949 & -3.07711 & -1.54335 \\ \mathrm{H} & 2.06916 & -3.72017 & -3.19573 \\ \mathrm{H} & -3.1182 & 3.75789 & -0.94393 \\ \mathrm{H} & -3.33918 & 4.01568 & -2.68205 \\ \mathrm{H} & -5.43087 & 4.91828 & -1.81871 \\ \mathrm{H} & -5.50774 & 3.66817 & -0.55357 \\ \mathrm{H} & -7.00455 & 2.74862 & -2.25308 \\ \mathrm{H} & -5.91853 & 3.24538 & -3.55814 \\ \mathrm{H} & -5.08491 & 1.0455 & -3.35061 \\ \mathrm{H} & -5.92122 & -0.79413 & -2.69908 \\ \mathrm{H} & -6.27925 & -3.1371 & -2.38371 \\ \mathrm{H} & -7.89148 & -2.41302 & -2.61361 \\ \mathrm{H} & -7.60337 & -3.55693 & -1.28497 \\ \mathrm{H} & -8.78482 & -0.74009 & -0.97903 \\ \mathrm{H} & -8.47712 & -1.82976 & 0.39989 \\ \mathrm{H} & -7.80514 & -0.18927 & 0.40145 \\ \mathrm{H} & -4.14754 & -1.40381 & -0.80115 \\ \mathrm{H} & -4.09484 & -3.11399 & 1.61869 \\ \mathrm{H} & -2.92562 & 0.89153 & 2.93309 \\ \mathrm{H} & -3.21073 & -0.03419 & 4.44085 \\ \mathrm{H} & -1.64403 & -0.16616 & 3.57483 \\ \mathrm{H} & -3.11479 & -4.3181 & -0.3005 \\ \mathrm{H} & -2.03311 & -2.96937 & -0.65058 \\ \mathrm{H} & -2.85382 & -5.4227 & 2.10711 \\ \mathrm{H} & -1.16248 & -6.30496 & 3.67868 \\ \mathrm{H} & 1.18217 & -5.48456 & 3.58672 \\ \mathrm{H} & 1.82351 & -3.76951 & 1.89011 \\ \mathrm{H} & 0.12843 & -2.92443 & 0.3008\end{array}$

\section{- $(S, S)-\mathrm{A} 2 \mathrm{~B} 1 \mathrm{C} 1 S i$}

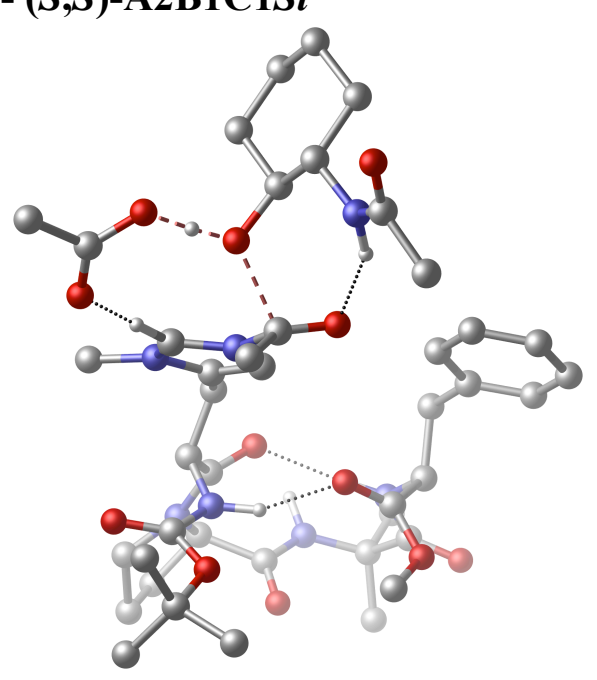


$\begin{array}{llll}\text { O } & -3.34367 & 1.07982 & 0.95129\end{array}$

$\begin{array}{llll}\text { C } & -6.06153 & -3.30817 & -0.89743\end{array}$

$\begin{array}{lllll}\text { O } & -4.8731 & -3.6662 & -0.74472\end{array}$

$\begin{array}{llll}\text { O } & -6.49613 & -2.09733 & -0.86524\end{array}$

C $\quad-7.13622-4.36035-1.13135$

$\begin{array}{llll}\mathrm{H} & 0.44939 & -3.57761 & 5.32084\end{array}$

$\mathrm{H} \quad 1.818 \quad-2.60651 \quad 4.74573$

H $\quad 0.15457 \quad-2.04261 \quad 4.48186$

H $\quad 2.72995 \quad-4.5466 \quad 3.41135$

H $\quad 1.40525 \quad-5.56936 \quad 3.99146$

$\mathrm{H} \quad 1.69283 \quad-5.40375 \quad 2.24679$

$\mathrm{H} \quad-1.31969-3.1899 \quad 2.77035$

$\mathrm{H} \quad-0.71844 \quad-4.60251 \quad 1.87308$

$\mathrm{H} \quad-1.08076 \quad-4.73209 \quad 3.6057$

$\mathrm{H} \quad \begin{array}{llll}1.92923 & -0.95942 & 0.75811\end{array}$

H $\quad 1.7898 \quad-2.82437 \quad-1.53106$

H $\quad 1.106290 .14242-1.62853$

$\mathrm{H} \quad 0.76085-1.04775-2.86735$

$\mathrm{H} \quad-0.76986-3.09811 \quad-3.13932$

$\mathrm{H} \quad-2.04612 \quad-3.9365 \quad-2.22521$

$\mathrm{H} \quad-0.36663 \quad-3.95713-1.61606$

$\mathrm{H} \quad-3.43148 \quad-2.61415-0.71416$

$\begin{array}{llll}\mathrm{H} & -0.95109 & 0.7422 & 0.07142\end{array}$

$\mathrm{H} \quad \begin{array}{llll}-5.17104 & -0.57711 & 1.99976\end{array}$

$\mathrm{H} \quad \begin{array}{llll}-3.59995 & -1.18743 & 2.57194\end{array}$

$\mathrm{H} \quad-4.45906 \quad-2.02007 \quad 1.2464$

$\mathrm{H} \quad-7.64431 \quad-4.16349 \quad-2.08041$

$\mathrm{H} \quad-7.89377 \quad-4.29166-0.34471$

H $\quad-6.70254 \quad-5.36095 \quad-1.14221$

$\mathrm{H} \quad-5.64359-1.19107 \quad-0.7612$

$\mathrm{H} \quad-4.52724 \quad 1.62345 \quad-1.23499$

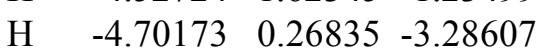

$\mathrm{H} \quad-6.35796 \quad-0.17754 \quad-2.89178$

$\begin{array}{lllll}\mathrm{H} & -6.37457 & 1.64537 & -4.58387\end{array}$

$\mathrm{H} \quad-5.36818 \quad 2.65517 \quad-3.55199$

$\mathrm{H} \quad-7.73972 \quad 3.35433 \quad-3.32232$

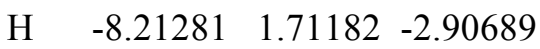

H $\quad-7.992693 .07236 \quad-0.82823$

$\begin{array}{lllll}\mathrm{H} & -6.33984 & 3.50968 & -1.26786\end{array}$

$\mathrm{H} \quad-7.37183 \quad 0.70516 \quad-0.615$

$\begin{array}{llll}\mathrm{H} & -5.26991 & 1.79378 & 1.03208\end{array}$

$\mathrm{H} \quad-5.58256 \quad 2.20779 \quad 3.20306$

$\mathrm{H} \quad-7.13324 \quad 1.5848 \quad 3.82703$

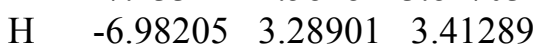

H $\quad 3.66946-3.66382-0.30952$

$\mathrm{H} \quad 4.06137 \quad-4.18441 \quad-1.95701$

H $\quad 6.118 \quad-4.77405 \quad-0.78959$

$\mathrm{H} \quad \begin{array}{llll}5.99843 & -3.33418 & 0.25093\end{array}$

$\mathrm{H} \quad \begin{array}{llll}7.58895 & -2.58953 & -1.44993\end{array}$

H $\quad 6.66125 \quad-3.36936-2.73826$

H $\quad 5.68174-1.23001-2.96496$ 


$\begin{array}{cccc}\mathrm{H} & 6.37448 & 0.73538 & -2.55015 \\ \mathrm{H} & 9.00608 & 1.17274 & -0.51646 \\ \mathrm{H} & 8.46359 & 2.45608 & 0.59813 \\ \mathrm{H} & 7.88089 & 0.79411 & 0.80914 \\ \mathrm{H} & 7.73623 & 3.80278 & -1.46226 \\ \mathrm{H} & 8.25292 & 2.4711 & -2.51952 \\ \mathrm{H} & 6.59119 & 3.10732 & -2.61983 \\ \mathrm{H} & 4.35921 & 1.48492 & -0.98138 \\ \mathrm{H} & 3.89112 & 3.64204 & 0.99709 \\ \mathrm{H} & 2.14084 & 2.7585 & -1.36405 \\ \mathrm{H} & 3.02814 & 4.27613 & -1.22469 \\ \mathrm{H} & 2.89649 & -0.05073 & 3.11654 \\ \mathrm{H} & 1.46603 & 0.98488 & 3.35531 \\ \mathrm{H} & 2.92177 & 1.22232 & 4.37795 \\ \mathrm{H} & 2.36615 & 5.79031 & 0.8932 \\ \mathrm{H} & 0.40726 & 6.76423 & 2.04254 \\ \mathrm{H} & -1.8252 & 5.68906 & 1.84284 \\ \mathrm{H} & -2.0812 & 3.62608 & 0.46271 \\ \mathrm{H} & -0.12518 & 2.69023 & -0.72038\end{array}$

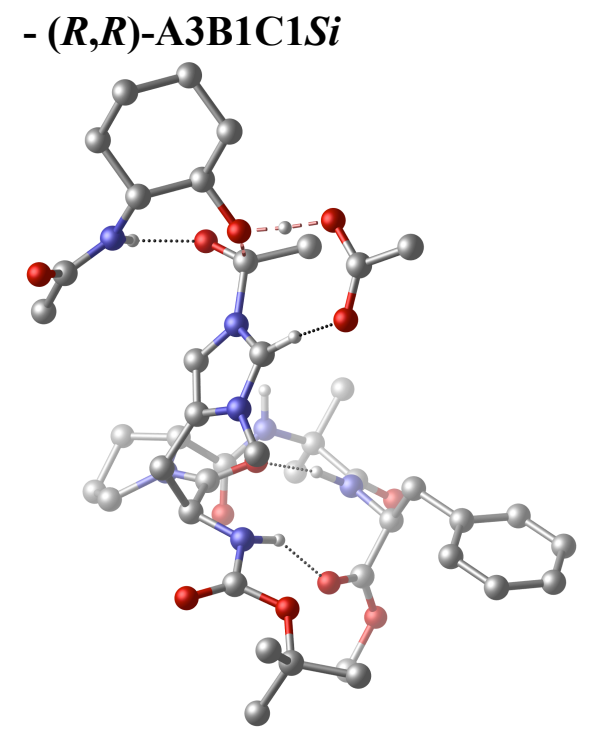

$\begin{array}{llll}\text { C } & 3.65089 & -3.79032 & -2.46059\end{array}$

$\mathrm{N} \quad 2.52597 \quad-3.88176 \quad-1.51541$

$\begin{array}{llll}\text { C } & 4.32235 & -2.3861 & -2.47487\end{array}$

$\begin{array}{lllll}\text { O } & 5.36275 & -2.22909 & -3.10419\end{array}$

$\begin{array}{llll}\text { C } & 3.07179 & -4.00943 & -3.8719\end{array}$

$\begin{array}{llll}\text { C } & 4.72019 & -4.85404 & -2.16032\end{array}$

$\begin{array}{llll}\text { C } & 1.75261 & 1.56081 & 3.04859\end{array}$

$\begin{array}{llll}\mathrm{O} & 1.32733 & 1.4241 & 4.18979\end{array}$

$\begin{array}{llll}\mathrm{O} & 2.43578 & 2.62488 & 2.57313\end{array}$

$\begin{array}{llll}\text { C } & 2.91194 & 3.68742 & 3.47991\end{array}$

$\begin{array}{llll}\text { C } & 3.85868 & 3.08877 & 4.52452\end{array}$

C $\quad 3.66743 \quad 4.61966 \quad 2.53075$

$\begin{array}{llll}\text { C } & 1.72294 & 4.41174 & 4.11907\end{array}$

$\begin{array}{lllll}\text { C } & 4.17177 & 4.62486 & -1.46991\end{array}$

$\begin{array}{llll}\text { C } & 3.6276 & 3.34689 & -1.32933\end{array}$

$\begin{array}{llll}\text { C } & 4.79651 & 4.99744 & -2.66045\end{array}$

$\begin{array}{lllll}\text { C } & 3.69854 & 2.42191 & -2.37977\end{array}$

$\begin{array}{llll}\text { C } & 4.87453 & 4.08336 & -3.71273\end{array}$

$\begin{array}{lllll}\text { C } & 3.13536 & 1.02268 & -2.23557\end{array}$

$\begin{array}{llll}\text { C } & 4.32939 & 2.80785 & -3.57098\end{array}$

$\begin{array}{llll}C & 4.20164 & -0.01697 & -1.83546\end{array}$

$\begin{array}{llll}\mathrm{N} & 3.65726 & -1.3702 & -1.85533\end{array}$

$\begin{array}{llll}\text { C } & 4.75066 & 0.25541 & -0.43164\end{array}$

$\begin{array}{lllll}\mathrm{O} & 4.07472 & 0.56463 & 0.53406\end{array}$

$\begin{array}{llll}\mathrm{O} & 6.07338 & 0.0681 & -0.37348\end{array}$

$\begin{array}{llll}\text { C } & 6.67431 & 0.21078 & 0.92696\end{array}$

$\begin{array}{lllll}\text { O } & 3.65208 & -3.75829 & 0.48128\end{array}$

$\begin{array}{llll}\text { C } & 2.60855 & -3.83902 & -0.15599\end{array}$

$\begin{array}{llll}\text { C } & -3.52084 & -0.74018 & -1.55784\end{array}$

$\begin{array}{lllll}\text { C } & -3.14831 & -0.19768 & -2.91969\end{array}$

$\begin{array}{llll}\text { O } & -3.88965 & -1.89601 & -1.37245\end{array}$

$\begin{array}{llll}\text { C } & -9.16546 & -0.26543 & -0.44894\end{array}$

$\begin{array}{lllll}\text { C } & -8.72423 & 0.02091 & -1.88934\end{array}$

$\begin{array}{llll}\text { C } & -8.12328 & -1.12428 & 0.27537\end{array}$

$\begin{array}{llll}\text { C } & -7.3372 & 0.67622 & -1.92024\end{array}$

$\begin{array}{llll}\text { C } & -6.71906 & -0.48545 & 0.2481\end{array}$

$\begin{array}{llll}\text { C } & -6.27509 & -0.16757 & -1.19424\end{array}$

$\begin{array}{lllll}\mathrm{O} & -5.00831 & 0.48262 & -1.19854\end{array}$

$\begin{array}{lllll}\mathrm{N} & -5.74246 & -1.36702 & 0.8843\end{array}$

$\begin{array}{llll}\text { C } & -5.66587 & -1.51027 & 2.23158\end{array}$

$\begin{array}{llll}\text { O } & -6.38417 & -0.90132 & 3.02226\end{array}$

$\begin{array}{lllll}\text { C } & -4.33036 & 3.57203 & -2.12384\end{array}$

$\begin{array}{lllll}\text { C } & -4.71967 & 4.85951 & -2.83423\end{array}$

$\begin{array}{lllll}\mathrm{O} & -3.41116 & 3.58039 & -1.28333\end{array}$

$\begin{array}{llll}\text { O } & -5.01752 & 2.53708 & -2.48099\end{array}$

$\begin{array}{llll}\text { C } & -4.61384 & -2.49861 & 2.72857\end{array}$

$\begin{array}{llll}\mathrm{H} & 4.47326 & 4.0831 & 2.02267\end{array}$

$\begin{array}{llll}\mathrm{H} & 4.09985 & 5.45389 & 3.0908\end{array}$

$\begin{array}{llll}\mathrm{H} & 2.9917 & 5.02711 & 1.77289\end{array}$

$\begin{array}{llll}\mathrm{H} & 4.67089 & 2.54497 & 4.03223\end{array}$

H $\quad 4.29985 \quad 3.89189 \quad 5.12311$

H $\quad 3.32752 \quad 2.40773 \quad 5.19019$

$\begin{array}{llll}\mathrm{H} & 2.08438 & 5.27417 & 4.68816\end{array}$

$\mathrm{H} \quad 1.17441 \quad 3.75097 \quad 4.79001$ 


\begin{tabular}{|c|c|c|c|}
\hline & & & \\
\hline & 2.16878 & 0.66594 & 1.24934 \\
\hline & 1.31012 & -0.95233 & \\
\hline & & & \\
\hline & & & \\
\hline I & & -2.01882 & \\
\hline & & & \\
\hline & & & \\
\hline & & & \\
\hline & & & \\
\hline & & & -2.94035 \\
\hline & & -0.65309 & -3.22163 \\
\hline & & & \\
\hline & & & \\
\hline & & & \\
\hline & & & \\
\hline & & & \\
\hline & & & \\
\hline & & 0.8 & \\
\hline & & & \\
\hline & & -0. & \\
\hline & & 0.6 & \\
\hline & & $2-0.7$ & \\
\hline & & -2.1 & \\
\hline & & -1.2 & \\
\hline & & & \\
\hline & & -1 & \\
\hline & & & \\
\hline & & & \\
\hline & & & \\
\hline & & & \\
\hline & & & \\
\hline & & & \\
\hline & & & \\
\hline & & & \\
\hline & & & \\
\hline & & -5.5 & \\
\hline & & & \\
\hline & & & \\
\hline & 2.2 & -3.2 & \\
\hline & 2.6 & -5.0 & \\
\hline & & & \\
\hline & & -5.8 & \\
\hline & & -4.7 & \\
\hline & & -4.7 & -1.14 \\
\hline & & & \\
\hline & & -0.0 & \\
\hline & & -0.5 & \\
\hline & & & \\
\hline & & & \\
\hline & & 0.68986 & -3.1875 \\
\hline & & 1.00239 & -1.49316 \\
\hline & & 2.1024 & \\
\hline & 5.35528 & 126171 & \\
\hline
\end{tabular}
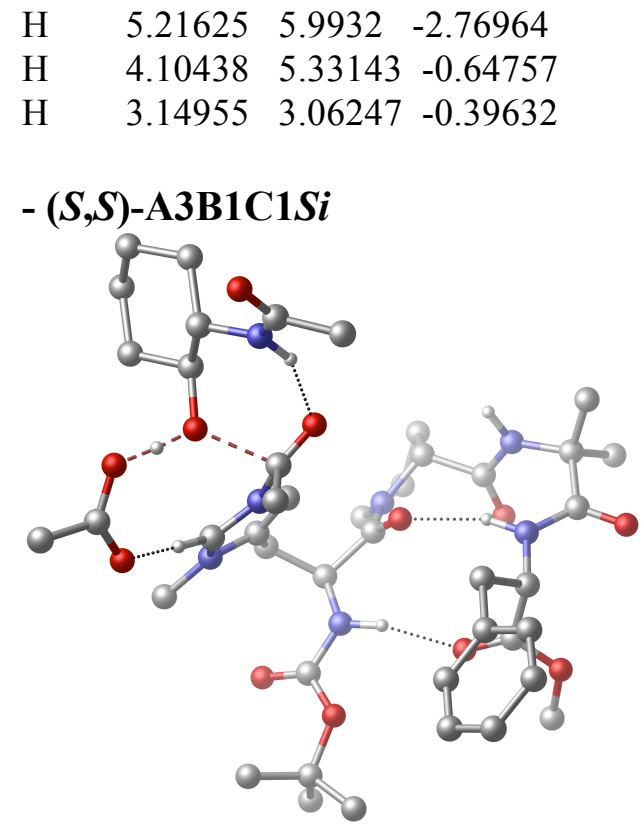

$\begin{array}{llll}\mathrm{N} & -1.97887 & 1.55858 & -1.6139\end{array}$

$\begin{array}{lllll}\text { C } & -1.7059 & 0.59391 & -2.65931\end{array}$

$\begin{array}{lllll}\text { C } & -0.23479 & 0.65803 & -3.19542\end{array}$

$\begin{array}{lllll}\text { C } & -1.99694 & -0.81444 & -2.13537\end{array}$

$\begin{array}{lllll}C & 0.8326 & 0.74963 & -2.1488\end{array}$

$\begin{array}{lllll}\mathrm{O} & -1.7238 & -1.12851 & -0.96814\end{array}$

$\mathrm{N} \quad 1.71343 \quad 1.83636 \quad-2.06741$

$\begin{array}{llll}\text { C } & 1.18786 & -0.08877 & -1.12922\end{array}$

$\begin{array}{lllll}\text { C } & 2.55549 & 1.65168 & -1.04402\end{array}$

$\begin{array}{llll}\mathrm{N} & 2.2414 & 0.49322 & -0.45719\end{array}$

$\mathrm{N} \quad-2.46658 \quad-1.72949-3.01613$

$\begin{array}{llll}\text { C } & -3.04885 & -1.49752 & -4.35445\end{array}$

$\begin{array}{llll}\text { C } & -2.5835 & -3.13281 & -2.58619\end{array}$

$\begin{array}{llll}\text { C } & -3.85194 & -2.77857 & -4.61585\end{array}$

$\begin{array}{llll}\text { C } & -3.05649 & -3.85656 & -3.8652\end{array}$

$\begin{array}{llll}\text { C } & 1.77322 & 2.99682 & -2.95944\end{array}$

$\begin{array}{llll}\text { C } & -3.92743 & -4.29373 & 0.8462\end{array}$

$\mathrm{N} \quad-3.17946-4.01484-0.3914$

$\begin{array}{llll}\text { C } & -4.12832 & -3.03004 & 1.73303\end{array}$

$\begin{array}{llll}\text { O } & -4.87558 & -3.09228 & 2.70287\end{array}$

$\begin{array}{llll}\text { C } & -3.0731 & -5.27206 & 1.67631\end{array}$

$\begin{array}{llll}\text { C } & -5.29805 & -4.91959 & 0.54137\end{array}$

$\begin{array}{llll}\text { C } & -2.0012 & 2.8864 & -1.91863\end{array}$

$\begin{array}{llll}\text { O } & -1.64221 & 3.34143 & -3.00415\end{array}$

$\begin{array}{lllll}\text { O } & -2.43867 & 3.60502 & -0.86429\end{array}$

$\begin{array}{llll}\text { C } & -2.65582 & 5.06023 & -0.96732\end{array}$

$\begin{array}{llll}\text { C } & -3.7179 & 5.35092 & -2.03221\end{array}$

$\begin{array}{llll}\text { C } & -3.17378 & 5.4161 & 0.42758\end{array}$

$\begin{array}{llll}\text { C } & -1.33361 & 5.77902 & -1.25249\end{array}$

$\begin{array}{llll}\text { C } & -2.03306 & 3.3864 & 3.93231\end{array}$

$\begin{array}{llll}\text { C } & -2.01076 & 2.31713 & 3.03467\end{array}$

$\begin{array}{llll}\text { C } & -2.08703 & 3.15197 & 5.30641\end{array}$

$\begin{array}{llll}\text { C } & -2.04005 & 0.99551 & 3.49995\end{array}$

$\begin{array}{llll}\text { C } & -2.11845 & 1.83926 & 5.78066\end{array}$ 


\begin{tabular}{|c|c|c|c|}
\hline & & & \\
\hline & & 0.7726 & \\
\hline & & -0.72438 & \\
\hline & & & \\
\hline & 4.29466 & & 1.49411 \\
\hline & 3.92709 & 0.9354 & 0.51549 \\
\hline & & 0.42101 & 2.01972 \\
\hline & 6.41553 & 1.32099 & 1.34299 \\
\hline & 4.72176 & -2.75297 & -1.5315 \\
\hline & -3.61198 & -3.26509 & -1.44308 \\
\hline & 6.06 & & -1.97392 \\
\hline & & & \\
\hline & & & -0 \\
\hline & 7.70 & & -1.3 \\
\hline & & & \\
\hline & & & -0.08022 \\
\hline & & & \\
\hline & & & \\
\hline & & & \\
\hline & & & \\
\hline & & & \\
\hline & & & \\
\hline & & & \\
\hline & 2.7 & & \\
\hline & 5.96 & & \\
\hline & 4.7 & & \\
\hline & & & \\
\hline & 28 & & \\
\hline & & & \\
\hline & & & \\
\hline & -2.4 & & \\
\hline & & & \\
\hline & & & \\
\hline & & & \\
\hline & -0.5 & & \\
\hline & & & \\
\hline & & & \\
\hline & & & \\
\hline & & & \\
\hline & -0.0 & & \\
\hline & -0.1 & & \\
\hline & & & \\
\hline & & & \\
\hline & & & \\
\hline & & & \\
\hline & & & \\
\hline & & & \\
\hline & & & \\
\hline & & & \\
\hline & & & \\
\hline & & & \\
\hline & & & -0.15042 \\
\hline & & 0.87891 & -0.04918 \\
\hline & 4.50722 & 1.94254 & -0.79793 \\
\hline
\end{tabular}

$\begin{array}{llll}\mathrm{H} & 5.35281 & -0.64204 & -2.71429 \\ \mathrm{H} & 6.81433 & -0.22252 & -1.82889 \\ \mathrm{H} & 7.30875 & -2.0882 & -3.39693 \\ \mathrm{H} & 6.00117 & -3.04965 & -2.71631 \\ \mathrm{H} & 8.16118 & -3.79259 & -1.74178 \\ \mathrm{H} & 8.52478 & -2.15043 & -1.22673 \\ \mathrm{H} & 7.62693 & -3.45526 & 0.70086 \\ \mathrm{H} & 6.1835 & -3.86764 & -0.22762 \\ \mathrm{H} & 7.03803 & -1.07086 & 0.66871 \\ \mathrm{H} & 4.4913 & -2.05599 & 1.58458 \\ \mathrm{H} & 4.08824 & -2.41873 & 3.75011 \\ \mathrm{H} & 5.37584 & -1.79113 & 4.81239 \\ \mathrm{H} & 5.32079 & -3.50907 & 4.43215 \\ \mathrm{H} & -3.68254 & -0.60811 & -4.35597 \\ \mathrm{H} & -2.25605 & -1.36034 & -5.1013 \\ \mathrm{H} & -3.95867 & -2.98747 & -5.68313 \\ \mathrm{H} & -4.84411 & -2.68147 & -4.16971 \\ \mathrm{H} & -3.64944 & -4.74561 & -3.63846 \\ \mathrm{H} & -2.18432 & -4.17325 & -4.44746 \\ \mathrm{H} & -1.60154 & -3.48661 & -2.25451 \\ \mathrm{H} & -2.20218 & -4.26972 & -0.38665 \\ \mathrm{H} & -5.16041 & -5.87622 & 0.02809 \\ \mathrm{H} & -5.82701 & -5.08492 & 1.48078 \\ \mathrm{H} & -5.8929 & -4.25975 & -0.09 \\ \mathrm{H} & -3.57296 & -5.46963 & 2.62561 \\ \mathrm{H} & -2.94455 & -6.2172 & 1.13926 \\ \mathrm{H} & -2.08478 & -4.85089 & 1.89442 \\ \mathrm{H} & -2.831 & -1.89109 & 0.57722 \\ \mathrm{H} & -3.96543 & -0.99382 & 3.1623 \\ \mathrm{H} & -1.56132 & 0.10113 & 1.59241 \\ \mathrm{H} & -1.45409 & -1.00393 & 2.96148 \\ \mathrm{H} & -6.57753 & 0.99247 & 0.31398 \\ \mathrm{H} & -6.00553 & 2.33345 & 1.33672 \\ \mathrm{H} & -7.34497 & 1.28457 & 1.90934 \\ \mathrm{H} & -2.11142 & -0.24772 & 5.25908 \\ \mathrm{H} & -2.15553 & 1.64596 & 6.84886 \\ \mathrm{H} & -2.10009 & 3.9849 & 6.00328 \\ \mathrm{H} & -2.00415 & 4.40572 & 3.55753 \\ \mathrm{H} & -1.97357 & 2.50839 & 1.96572 \\ & & & \\ & & & \end{array}$




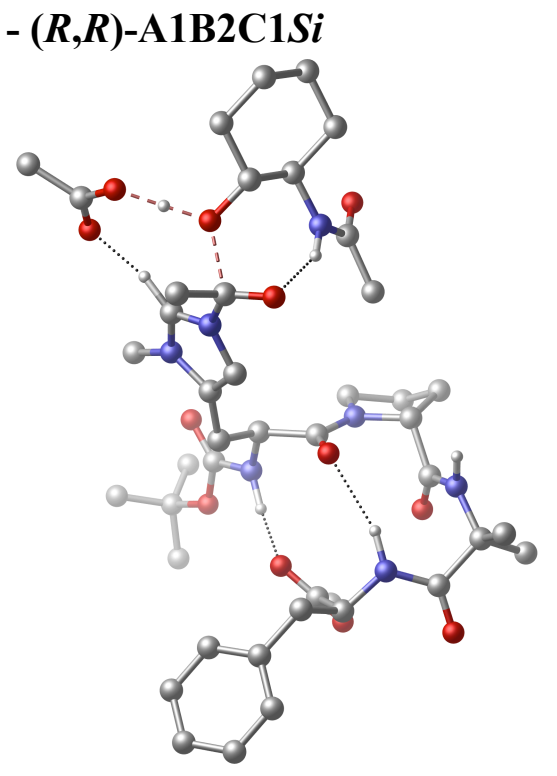

$\begin{array}{llll}\mathrm{N} & -1.8328 & 1.66729 & -1.51865 \\ \mathrm{C} & -0.64232 & 1.00746 & -1.00496 \\ \mathrm{C} & -0.23593 & 1.55409 & 0.38677 \\ \mathrm{C} & -0.88429 & -0.51652 & -0.94087 \\ \mathrm{C} & 1.18996 & 1.30802 & 0.76024 \\ \mathrm{O} & -1.01829 & -1.10414 & 0.14222 \\ \mathrm{~N} & 2.21999 & 2.19446 & 0.43213 \\ \mathrm{C} & 1.78672 & 0.28709 & 1.4456 \\ \mathrm{C} & 3.38002 & 1.73073 & 0.91602 \\ \mathrm{~N} & 3.13776 & 0.57456 & 1.53966 \\ \mathrm{~N} & -0.93933 & -1.18563 & -2.11478 \\ \mathrm{C} & -0.84272 & -0.63577 & -3.48132 \\ \mathrm{C} & -1.15663 & -2.63884 & -2.10311 \\ \mathrm{C} & -1.44865 & -1.75003 & -4.34176 \\ \mathrm{C} & -1.04568 & -3.03087 & -3.59442 \\ \mathrm{C} & 2.09783 & 3.46875 & -0.28461 \\ \mathrm{C} & -3.72585 & -4.47046 & 0.11121 \\ \mathrm{~N} & -2.53168 & -3.93017 & -0.55696 \\ \mathrm{C} & -4.54779 & -3.36108 & 0.82575 \\ \mathrm{O} & -5.72948 & -3.54534 & 1.08396 \\ \mathrm{C} & -3.24212 & -5.44597 & 1.2008 \\ \mathrm{C} & -4.63134 & -5.19536 & -0.89726 \\ \mathrm{C} & -1.69996 & 2.86597 & -2.16953 \\ \mathrm{O} & -0.62484 & 3.33943 & -2.52056 \\ \mathrm{O} & -2.91834 & 3.40723 & -2.37964 \\ \mathrm{C} & -3.07329 & 4.69197 & -3.08978 \\ \mathrm{C} & -2.54814 & 4.56211 & -4.52252 \\ \mathrm{C} & -4.59031 & 4.89195 & -3.08041 \\ \mathrm{C} & -2.37296 & 5.80898 & -2.31012 \\ \mathrm{C} & -5.24247 & 2.91252 & 4.04683 \\ \mathrm{C} & -4.47149 & 1.92225 & 3.43595 \\ \mathrm{C} & -6.27687 & 2.56145 & 4.91481 \\ \mathrm{C} & -4.71946 & 0.56599 & 3.68461 \\ \mathrm{C} & -6.53571 & 1.21319 & 5.16855 \\ \mathrm{C} & -3.90169 & -0.51996 & 3.01496\end{array}$

\begin{tabular}{|c|c|c|}
\hline$\sigma^{2}$ & -5.7623 & 4.55819 \\
\hline & $-4.57214-1.08791$ & 1.75287 \\
\hline & $-3.85647-2.25087$ & 1.23133 \\
\hline & $-4.61661-0.07823$ & 0.60344 \\
\hline & $-3.7458 \quad 0.7542$ & 0.3965 \\
\hline & $\begin{array}{ll}-5.66371 & -0.28339\end{array}$ & -0.19209 \\
\hline 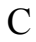 & $\begin{array}{ll}-5.6615 & 0.40641\end{array}$ & -1.45859 \\
\hline & $\begin{array}{ll}-3.5643 & -2.43198\end{array}$ & -1.94142 \\
\hline & $-2.54333-2.97473$ & -1.5263 \\
\hline $\mathrm{C}$ & $4.10482-0.29922$ & 2.31808 \\
\hline $\mathrm{C}$ & $4.65814 \quad 0.39745$ & 3.54189 \\
\hline $\mathrm{O}$ & $3.85486-1.49783$ & 2.28223 \\
\hline $\mathrm{C}$ & $\begin{array}{lll}8.43171 & -2.56927\end{array}$ & -0.74277 \\
\hline $\mathrm{C}$ & $\begin{array}{ll}8.7932 & -1.95099\end{array}$ & 0.61393 \\
\hline $\mathrm{C}$ & $6.93958-2.91582$ & -0.803 \\
\hline $\mathrm{C}$ & $\begin{array}{ll}7.91601 & -0.72814\end{array}$ & 0.91532 \\
\hline $\mathrm{C}$ & $6.049-1.69418$ & -0.49826 \\
\hline $\mathrm{C}$ & $6.41678-1.06435$ & 0.8596 \\
\hline $\mathrm{O}$ & $\begin{array}{ll}5.6151 & 0.08564\end{array}$ & 1.11312 \\
\hline $\mathrm{N}$ & $4.63644-2.06352$ & -0.49727 \\
\hline $\mathrm{C}$ & $\begin{array}{lll}3.94022 & -2.28199\end{array}$ & -1.63834 \\
\hline $\mathrm{O}$ & $4.42709-2.20673$ & -2.76551 \\
\hline $\mathrm{C}$ & $6.66077 \quad 3.18594$ & 1.16996 \\
\hline $\mathrm{C}$ & 7.74703 & 1.37466 \\
\hline $\mathrm{O}$ & 5.64286 & 0.5101 \\
\hline $\mathrm{O}$ & 6.90329 & 1.72953 \\
\hline $\mathrm{C}$ & $2.47584-2.66262$ & -1.43662 \\
\hline $\mathrm{H}$ & $-5.09168 \quad 4.07088$ & -3.6007 \\
\hline $\mathrm{H}$ & -4.84808 & -3.58359 \\
\hline $\mathrm{H}$ & -4.96825 & -2.05496 \\
\hline $\mathrm{H}$ & -3.04218 & -5.03456 \\
\hline $\mathrm{H}$ & $-2.76749 \quad 5.47946$ & -5.07791 \\
\hline $\mathrm{H}$ & $-1.47084 \quad 4.39525$ & -4.53196 \\
\hline $\mathrm{H}$ & -2.59348 & -2.77644 \\
\hline $\mathrm{H}$ & $-1.2927 \quad 5.66324$ & -2.30093 \\
\hline $\mathrm{H}$ & -2.7391 & -1.2791 \\
\hline $\mathrm{H}$ & -2.711331 .4772 & -1.03817 \\
\hline $\mathrm{H}$ & 0.15671 & -1.72311 \\
\hline $\mathrm{H}$ & $-0.42515 \quad 2.63102$ & 0.38238 \\
\hline $\mathrm{H}$ & $\begin{array}{ll}-0.89207 & 1.10259\end{array}$ & 1.13226 \\
\hline $\mathrm{H}$ & $1.36765-0.6109$ & 1.86708 \\
\hline $\mathrm{H}$ & 1.58318 & 0.34568 \\
\hline $\mathrm{H}$ & 3.10446 & -0.49911 \\
\hline $\mathrm{H}$ & 1.53106 & -1.20778 \\
\hline $\mathrm{H}$ & 4.34151 & 0.77975 \\
\hline $\mathrm{H}$ & 5.05082 & 3.32612 \\
\hline $\mathrm{H}$ & 3.85634 & 4.28636 \\
\hline $\mathrm{H}$ & $\begin{array}{lll}5.45515 & -0.22257\end{array}$ & 3.95458 \\
\hline $\mathrm{H}$ & $7.43136 \quad 5.1983$ & 0.98124 \\
\hline $\mathrm{H}$ & 7.98754 & 2.43834 \\
\hline $\mathrm{H}$ & 8.66115 & 0.8647 \\
\hline $\mathrm{H}$ & 6.22386 & 1.39087 \\
\hline $\mathrm{H}$ & $8.12335 \quad 0.06381$ & 0.18481 \\
\hline $\mathrm{H}$ & $\begin{array}{lll}8.15324 & -0.30657\end{array}$ & 1.89691 \\
\hline
\end{tabular}




\begin{tabular}{|c|c|c|}
\hline $\mathrm{H}$ & $9.85048-1.66168$ & 0.63874 \\
\hline $\mathrm{H}$ & $8.65779-2.7042$ & 1.40319 \\
\hline $\mathrm{H}$ & $8.67443-1.85644$ & -1.54319 \\
\hline $\mathrm{H}$ & $9.03435-3.4657$ & -0.93115 \\
\hline $\mathrm{H}$ & $6.71356-3.70188$ & -0.06875 \\
\hline $\mathrm{H}$ & $6.66199-3.30313$ & -1.78736 \\
\hline $\mathrm{H}$ & $\begin{array}{lll}6.19917 & -0.94218\end{array}$ & -1.28323 \\
\hline $\mathrm{H}$ & $6.19337-1.81362$ & 1.63845 \\
\hline $\mathrm{H}$ & $\begin{array}{lll}4.16309 & -2.08641\end{array}$ & 0.40142 \\
\hline $\mathrm{H}$ & $1.86805-2.05515$ & -2.11144 \\
\hline $\mathrm{H}$ & $2.12252-2.52818$ & -0.41064 \\
\hline $\mathrm{H}$ & $2.34389-3.71089$ & -1.7245 \\
\hline $\mathrm{H}$ & $\begin{array}{ll}-1.38852 & 0.3049\end{array}$ & -3.55348 \\
\hline $\mathrm{H}$ & $0.20795-0.45521$ & -3.74449 \\
\hline $\mathrm{H}$ & $\begin{array}{lll}-1.08266 & -1.72312\end{array}$ & -5.37105 \\
\hline $\mathrm{H}$ & $-2.53755-1.65707$ & -4.34899 \\
\hline $\mathrm{H}$ & $-1.67653-3.88762$ & -3.84252 \\
\hline $\mathrm{H}$ & $-0.00815 \quad-3.29885$ & -3.81947 \\
\hline $\mathrm{H}$ & $-0.37639-3.11271$ & -1.49947 \\
\hline $\mathrm{H}$ & $-1.63162-4.2368$ & -0.21743 \\
\hline $\mathrm{H}$ & $-2.587 \quad-4.94467$ & 1.92121 \\
\hline $\mathrm{H}$ & $-2.69975-6.28461$ & 0.75136 \\
\hline $\mathrm{H}$ & $-4.10373-5.84214$ & 1.74157 \\
\hline $\mathrm{H}$ & $-4.09733-6.05063$ & -1.32228 \\
\hline $\mathrm{H}$ & $\begin{array}{lll}-5.53389 & -5.54229\end{array}$ & -0.39332 \\
\hline $\mathrm{H}$ & $-4.92215-4.51776$ & -1.70114 \\
\hline $\mathrm{H}$ & $-2.91185-2.09526$ & 0.89154 \\
\hline $\mathrm{H}$ & $\begin{array}{lll}-5.58791 & -1.42575\end{array}$ & 1.96548 \\
\hline $\mathrm{H}$ & $-4.89759-0.03601$ & -2.10216 \\
\hline $\mathrm{H}$ & $-6.6524 \quad 0.2421$ & -1.87934 \\
\hline $\mathrm{H}$ & $\begin{array}{lll}-5.47012 & 1.47237\end{array}$ & -1.32049 \\
\hline $\mathrm{H}$ & $\begin{array}{ll}-3.7584 & -1.3594\end{array}$ & 3.70295 \\
\hline $\mathrm{H}$ & $\begin{array}{ll}-2.90978 & -0.14399\end{array}$ & 2.74588 \\
\hline $\mathrm{H}$ & $\begin{array}{ll}-5.96525 & -0.82177\end{array}$ & 4.76583 \\
\hline $\mathrm{H}$ & $\begin{array}{ll}-7.33666 & 0.9297\end{array}$ & 5.84528 \\
\hline $\mathrm{H}$ & $\begin{array}{ll}-6.87543 & 3.33179\end{array}$ & 5.39218 \\
\hline $\mathrm{H}$ & $-5.03283 \quad 3.95936$ & 3.84559 \\
\hline $\mathrm{H}$ & $-3.67352 \quad 2.19883$ & 2.75345 \\
\hline
\end{tabular}

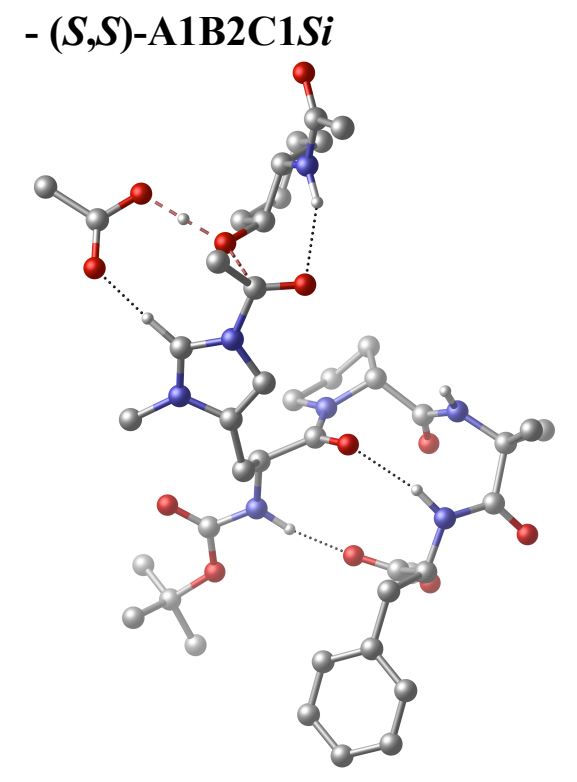

$\begin{array}{llll}\mathrm{N} & 2.24877 & 1.75585 & 0.64924 \\ \mathrm{C} & 0.91118 & 1.35565 & 0.25273 \\ \mathrm{C} & 0.56497 & 1.79744 & -1.1937 \\ \mathrm{C} & 0.75794 & -0.17491 & 0.38126 \\ \mathrm{C} & -0.90369 & 1.74042 & -1.46101 \\ \mathrm{O} & 0.85797 & -0.90761 & -0.61578 \\ \mathrm{~N} & -1.77162 & 2.8092 & -1.2027 \\ \mathrm{C} & -1.69706 & 0.70879 & -1.8747 \\ \mathrm{C} & -3.02966 & 2.43215 & -1.46356 \\ \mathrm{~N} & -3.00091 & 1.16234 & -1.88179 \\ \mathrm{~N} & 0.47348 & -0.67303 & 1.60152 \\ \mathrm{C} & 0.4347 & 0.06619 & 2.87832 \\ \mathrm{C} & 0.12681 & -2.09952 & 1.75928 \\ \mathrm{C} & 0.48144 & -1.05468 & 3.9219 \\ \mathrm{C} & -0.28854 & -2.19908 & 3.24368 \\ \mathrm{C} & -1.39752 & 4.15706 & -0.76182 \\ \mathrm{C} & 2.13802 & -4.78441 & -0.14804 \\ \mathrm{~N} & 1.13801 & -3.83816 & 0.37484 \\ \mathrm{C} & 3.46448 & -4.07047 & -0.53719 \\ \mathrm{O} & 4.5163 & -4.69348 & -0.57235 \\ \mathrm{C} & 1.55513 & -5.40627 & -1.43104 \\ \mathrm{C} & 2.44132 & -5.87644 & 0.88914 \\ \mathrm{C} & 2.4858 & 3.06433 & 0.9579 \\ \mathrm{O} & 1.61028 & 3.92577 & 0.97789 \\ \mathrm{O} & 3.79449 & 3.24853 & 1.22406 \\ \mathrm{C} & 4.32098 & 4.57089 & 1.61341 \\ \mathrm{C} & 3.66499 & 5.03173 & 2.9187 \\ \mathrm{C} & 5.80856 & 4.2827 & 1.82562 \\ \mathrm{C} & 4.11426 & 5.57152 & 0.47258 \\ \mathrm{C} & 6.97999 & 1.92285 & -2.10698 \\ \mathrm{C} & 5.81275 & 1.19972 & -1.8553 \\ \mathrm{C} & 7.97046 & 1.3949 & -2.93549 \\ \mathrm{C} & 5.61588 & -0.06231 & -2.43183 \\ \mathrm{C} & 7.78636 & 0.13745 & -3.51356 \\ \mathrm{C} & 4.36181 & -0.86728 & -2.15651 \\ & & & \\ & & & \end{array}$




\begin{tabular}{|c|c|c|c|}
\hline & & & \\
\hline & & & \\
\hline & 3.35694 & -2.75858 & \\
\hline & & & \\
\hline & 4.24409 & -0.30664 & \\
\hline & 5.65252 & -2.03127 & \\
\hline & & & \\
\hline & 2.34508 & -2.9 & \\
\hline & & & \\
\hline & & & \\
\hline & & & \\
\hline & -5.0 & -0.4 & \\
\hline C & -6.1 & & \\
\hline $\mathrm{C}$ & -6.3 & & \\
\hline & -6.3 & & \\
\hline 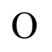 & -4.9 & & \\
\hline & -6.46028 & & \\
\hline & -7.6 & & \\
\hline $\mathrm{O}$ & -8.6 & & -0 \\
\hline C & -7.5 & & \\
\hline C & -4.1 & & \\
\hline C & -5.2 & & \\
\hline $\mathrm{O}$ & -3.9 & & \\
\hline C & -6.4 & & \\
\hline $\mathrm{O}$ & -5.4 & & \\
\hline $\mathrm{O}$ & -6.6 & & \\
\hline $\mathrm{C}$ & -7.4 & & \\
\hline $\mathrm{H}$ & & & \\
\hline $\mathrm{H}$ & & & \\
\hline $\mathrm{H}$ & 6.2 & & 0.9 \\
\hline $\mathrm{H}$ & & & \\
\hline $\mathrm{H}$ & 4.1 & & \\
\hline $\mathrm{H}$ & 2.6 & & 2.7 \\
\hline $\mathrm{H}$ & 4.5 & & \\
\hline $\mathrm{H}$ & & & \\
\hline $\mathrm{H}$ & & & \\
\hline $\mathrm{H}$ & & & \\
\hline $\mathrm{H}$ & & & \\
\hline $\mathrm{H}$ & & & -1.3 \\
\hline $\mathrm{H}$ & & & -1.8 \\
\hline $\mathrm{H}$ & -0.6 & 4.1 & 0.02 \\
\hline $\mathrm{H}$ & -2.2 & & -0.35 \\
\hline $\mathrm{H}$ & -0.98872 & 4.72 & -1.60 \\
\hline $\mathrm{H}$ & -3.93776 & 3.02839 & -1.2834 \\
\hline $\mathrm{H}$ & -1.45395 & -0.3047 & -2.1432 \\
\hline $\mathrm{H}$ & -6.19502 & 0.39646 & -2.822 \\
\hline $\mathrm{H}$ & -4.99874 & 1.00413 & -3.9917 \\
\hline $\mathrm{H}$ & -5.46782 & 2.00004 & -2.5866 \\
\hline $\mathrm{H}$ & & & 1.2713 \\
\hline $\mathrm{H}$ & -8.44026 & 4.31065 & -0.281( \\
\hline $\mathrm{H}$ & -7.19087 & 5.57343 & -0.0925 \\
\hline $\mathrm{H}$ & -5.82294 & 1.50614 & $-0.156 \mathrm{~S}$ \\
\hline $\mathrm{H}$ & -4.18943 & -1.07968 & 0.2892 \\
\hline & -3.85244 & 0.64972 & 2.02 \\
\hline
\end{tabular}

$\begin{array}{llll}\mathrm{H} & -5.58984 & 0.84357 & 2.20982 \\ \mathrm{H} & -4.71893 & -0.5931 & 4.0443 \\ \mathrm{H} & -3.98547 & -1.69093 & 2.87805 \\ \mathrm{H} & -6.1399 & -2.64475 & 3.65927 \\ \mathrm{H} & -6.98736 & -1.17216 & 3.20438 \\ \mathrm{H} & -7.28971 & -2.89637 & 1.42846 \\ \mathrm{H} & -5.54027 & -3.0749 & 1.27709 \\ \mathrm{H} & -7.17781 & -0.57038 & 0.64376 \\ \mathrm{H} & -5.64343 & -1.7511 & -1.49026 \\ \mathrm{H} & -6.63074 & -2.62073 & -3.29464 \\ \mathrm{H} & -8.37646 & -2.26439 & -3.37544 \\ \mathrm{H} & -7.83438 & -3.83988 & -2.80769 \\ \mathrm{H} & 1.27984 & 0.75391 & 2.94555 \\ \mathrm{H} & -0.49662 & 0.64319 & 2.95637 \\ \mathrm{H} & 0.04246 & -0.74915 & 4.87483 \\ \mathrm{H} & 1.51651 & -1.36206 & 4.08782 \\ \mathrm{H} & -0.05261 & -3.17694 & 3.66768 \\ \mathrm{H} & -1.36893 & -2.04137 & 3.32824 \\ \mathrm{H} & -0.70667 & -2.33111 & 1.0878 \\ \mathrm{H} & 0.34028 & -3.6417 & -0.21278 \\ \mathrm{H} & 1.53129 & -6.44664 & 1.09791 \\ \mathrm{H} & 3.21042 & -6.54505 & 0.50091 \\ \mathrm{H} & 2.80606 & -5.43071 & 1.81531 \\ \mathrm{H} & 2.28401 & -6.09384 & -1.86482 \\ \mathrm{H} & 0.64197 & -5.96615 & -1.20436 \\ \mathrm{H} & 1.3239 & -4.63907 & -2.17781 \\ \mathrm{H} & 2.47708 & -2.26907 & -0.77845 \\ \mathrm{H} & 5.37768 & -2.60063 & -1.29674 \\ \mathrm{H} & 3.53995 & -0.20657 & -1.86358 \\ \mathrm{H} & 4.04518 & -1.3955 & -3.06181 \\ \mathrm{H} & 4.80054 & -1.80545 & 2.92187 \\ \mathrm{H} & 6.09146 & -0.63047 & 2.53223 \\ \mathrm{H} & 6.51017 & -2.34806 & 2.84724 \\ \mathrm{H} & 6.47881 & -1.55758 & -3.72273 \\ \mathrm{H} & 8.54964 & -0.28125 & -4.16307 \\ \mathrm{H} & 8.87744 & 1.95908 & -3.13212 \\ \mathrm{H} & 7.11387 & 2.90269 & -1.65679 \\ \mathrm{H} & 5.05219 & 1.6173 & -1.20198\end{array}$




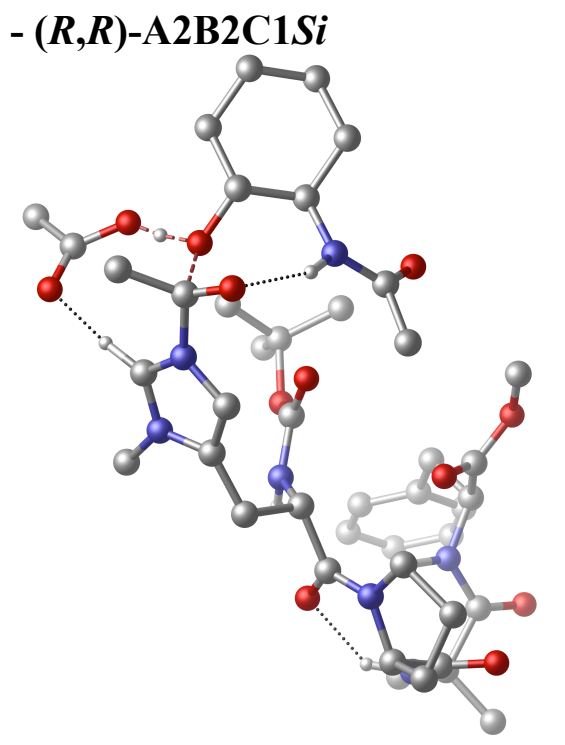

$\mathrm{N} \quad 0.48711 \quad-0.31818-1.40034$

C $\quad 0.64886 \quad-1.70646 \quad-1.01479$

$\begin{array}{lllll}\text { C } & -0.23033 & -2.65734 & -1.88979\end{array}$

C $\quad 2.12348 \quad-2.08538 \quad-1.20574$

C $\quad-1.68836-2.32511 \quad-1.83514$

O $\quad 2.78786-1.49466-2.07681$

$\mathrm{N} \quad-2.32736 \quad-1.47759 \quad-2.74394$

C $\quad-2.64302-2.6715 \quad-0.92122$

C $\quad-3.60805-1.32507-2.3798$

$\mathrm{N} \quad \begin{array}{llll}-3.81636 & -2.04336 & -1.27589\end{array}$

$\mathrm{N} \quad 2.62524 \quad-3.09352 \quad-0.46705$

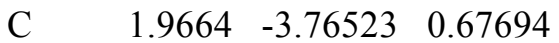

$\begin{array}{lllll}\text { C } & 3.98714 & -3.62373 & -0.71794\end{array}$

$\begin{array}{llll}\text { C } & 3.04818 & -4.71566 & 1.21611\end{array}$

$\begin{array}{llll}\text { C } & 3.96009 & -4.97547 & 0.00562\end{array}$

$\begin{array}{lllll}\text { C } & -1.74558 & -0.84116 & -3.92578\end{array}$

$\begin{array}{lllll}\text { C } & 6.36553 & -0.58612 & -0.59759\end{array}$

$\begin{array}{lllll}\mathrm{N} & 5.4454 & -1.67572 & -0.96843\end{array}$

$\begin{array}{llll}\text { C } & 5.79352 & 0.26742 & 0.57038\end{array}$

$\begin{array}{lllll}\mathrm{O} & 6.52954 & 0.84099 & 1.36311\end{array}$

$\begin{array}{llll}\text { C } & 6.48169 & 0.34514 & -1.82097\end{array}$

$\begin{array}{lllll}\text { C } & & 7.74761 & -1.13002 & -0.21149\end{array}$

$\begin{array}{llll}\text { C } & -0.32265 & 0.511 & -0.65456\end{array}$

$\begin{array}{lllll}\mathrm{O} & -1.11998 & 0.09999 & 0.17012\end{array}$

$\begin{array}{lllll}\mathrm{O} & -0.12012 & 1.7953 & -1.01364\end{array}$

C $\quad-1.03641 \quad 2.86558 \quad-0.53215$

$\begin{array}{llll}\text { C } & -0.94081 & 3.00942 & 0.98848\end{array}$

$\begin{array}{lllll}\text { C } & -0.48132 & 4.10482 & -1.23711\end{array}$

$\begin{array}{lllll}\text { C } & -2.46167 & 2.56375 & -1.00013\end{array}$

C $\quad 6.11006 \quad 4.67067 \quad-0.07623$

$\begin{array}{llll}\text { C } & 5.24572 & 3.90111 & 0.70101\end{array}$

C $\quad 5.831 \quad 4.89853 \quad-1.42583$

$\begin{array}{llll}\mathrm{C} & 4.08733 & 3.34222 & 0.14331\end{array}$

$\begin{array}{lllll}C & 4.68155 & 4.34851 & -1.99258\end{array}$

$\begin{array}{lllll}\text { C } & 3.13028 & 2.51273 & 0.97263\end{array}$

$\begin{array}{lllll}\text { C } & 3.81987 & 3.57481 & -1.21201\end{array}$

$\mathrm{C}$

$\mathrm{N}$

C

$\mathrm{O}$

$\mathrm{O}$

C

$\mathrm{O}$

C

$\mathrm{C}$

$\mathrm{C}$

$\mathrm{O}$

C

$\mathrm{C}$

C

C

C

C

O

N

C

O $\begin{array}{lll}3.75393 & 1.21329 & 1.56353\end{array}$

$\begin{array}{lll}4.43509 & 0.41201 & 0.57272\end{array}$

$\begin{array}{llll}2.63989 & 0.39983 & 2.22124\end{array}$

$\begin{array}{lll}2.11795 & -0.58124 & 1.71887\end{array}$

$\begin{array}{lll}2.28528 & 0.94303 & 3.38648\end{array}$

$\begin{array}{llll}1.18602 & 0.32945 & 4.10408\end{array}$

$\begin{array}{llll}5.53485 & -2.85896 & 0.99224\end{array}$

$\begin{array}{llll}5.07317 & -2.68052 & -0.1298\end{array}$

$\begin{array}{llll}-5.09999 & -2.16239 & -0.47591\end{array}$

$\begin{array}{llll}-6.32775 & -2.25729 & -1.35532\end{array}$

$\begin{array}{lll}-4.97782 & -2.77163 & 0.58251\end{array}$

$\begin{array}{lll}-6.44511 & 1.74108 & 3.50253\end{array}$

$\begin{array}{lll}-7.51748 & 1.3251 & 2.48707\end{array}$

$\begin{array}{lll}-5.35028 & 0.67338 & 3.60228\end{array}$

$\begin{array}{lll}-6.89174 & 1.0408 & 1.11447\end{array}$

$\begin{array}{lll}-4.7115 & 0.36528 & 2.23247\end{array}$

$\begin{array}{llll}-5.78226 & -0.02273 & 1.18924\end{array}$

$\begin{array}{llll}-5.19372 & -0.26875 & -0.083\end{array}$

$\begin{array}{lll}-3.74256 & -0.72129 & 2.3467\end{array}$

$\begin{array}{lll}-2.5138 & -0.56472 & 2.88439\end{array}$

$\begin{array}{lll}-2.11129 & 0.49882 & 3.37089\end{array}$

$\begin{array}{llll}-5.80477 & 1.44909 & -2.88397\end{array}$

$\begin{array}{lll}-6.31872 & 2.71209 & -3.55535\end{array}$

$\begin{array}{llll}-5.58064 & 0.42835 & -3.56204\end{array}$

$\begin{array}{llll}-5.64278 & 1.55888 & -1.60543\end{array}$

$\begin{array}{lll}-1.63337 & -1.8062 & 2.88157\end{array}$

$\begin{array}{llll}0.55222 & 4.29828 & -0.93562\end{array}$

$\begin{array}{llll}-1.08417 & 4.97958 & -0.97713\end{array}$

$\begin{array}{llll}-0.50766 & 3.97921 & -2.32371\end{array}$

$\begin{array}{lll}0.09694 & 3.17726 & 1.29235\end{array}$

$\begin{array}{llll}-1.52181 & 3.88434 & 1.29869\end{array}$

$\begin{array}{lll}-1.32909 & 2.13497 & 1.51172\end{array}$

$\begin{array}{llll}-3.11651 & 3.40259 & -0.74699\end{array}$

$\begin{array}{llll}-2.86524 & 1.66804 & -0.52833\end{array}$

$\begin{array}{lll}-2.48934 & 2.44019 & -2.08795\end{array}$

$\begin{array}{llll}1.25305 & 0.08026 & -1.92983\end{array}$

$\begin{array}{llll}0.34649 & -1.80587 & 0.02805\end{array}$

$\begin{array}{llll}0.13489 & -2.6106 & -2.92099\end{array}$

$\begin{array}{llll}-0.0908 & -3.6892 & -1.55199\end{array}$

$-2.59913-3.29578-0.04522$

$\begin{array}{llll}-2.51897 & -0.23314 & -4.39535\end{array}$

$\begin{array}{llll}-0.9067 & -0.21186 & -3.62759\end{array}$

$\begin{array}{llll}-1.40527 & -1.60022 & -4.63458\end{array}$

$\begin{array}{llll}-4.35239 & -0.69391 & -2.88463\end{array}$

$\begin{array}{llll}-6.34018 & -1.52181 & -2.16033\end{array}$

$-6.37013-3.26742-1.78016$

$-7.20455-2.11804-0.72151$

$\begin{array}{llll}-6.33444 & 2.59161 & -4.63886\end{array}$

$\begin{array}{lll}-7.33018 & 2.92792 & -3.1966\end{array}$

$\begin{array}{llll}-5.69238 & 3.56464 & -3.27787\end{array}$

$\begin{array}{llll}-5.41843 & 0.60361 & -0.92319\end{array}$

$\begin{array}{lll}-6.4716 & 1.96508 & 0.70228\end{array}$

$\begin{array}{lll}-7.65268 & 0.70517 & 0.40067\end{array}$

$\begin{array}{lll}-8.28363 & 2.10377 & 2.39068\end{array}$ 


$\begin{array}{llll}\mathrm{H} & -8.03155 & 0.42509 & 2.85349 \\ \mathrm{H} & -6.00045 & 2.6965 & 3.19043 \\ \mathrm{H} & -6.89332 & 1.91352 & 4.48824 \\ \mathrm{H} & -5.7801 & -0.25674 & 3.99971 \\ \mathrm{H} & -4.55648 & 0.98055 & 4.28904 \\ \mathrm{H} & -4.18287 & 1.25927 & 1.87811 \\ \mathrm{H} & -6.23099 & -0.96883 & 1.53504 \\ \mathrm{H} & -3.9886 & -1.59877 & 1.89836 \\ \mathrm{H} & -0.72078 & -1.56961 & 2.32993 \\ \mathrm{H} & -2.11698 & -2.68103 & 2.43816 \\ \mathrm{H} & -1.35518 & -2.0454 & 3.91221 \\ \mathrm{H} & 1.65323 & -3.02564 & 1.41649 \\ \mathrm{H} & 1.08656 & -4.32125 & 0.33017 \\ \mathrm{H} & 2.61412 & -5.62896 & 1.63059 \\ \mathrm{H} & 3.62816 & -4.21639 & 1.99418 \\ \mathrm{H} & 4.96091 & -5.29316 & 0.30038 \\ \mathrm{H} & 3.52848 & -5.73387 & -0.65683 \\ \mathrm{H} & 4.12871 & -3.71448 & -1.79883 \\ \mathrm{H} & 4.84967 & -1.54065 & -1.77949 \\ \mathrm{H} & 5.50455 & 0.73553 & -2.12203 \\ \mathrm{H} & 6.92444 & -0.19407 & -2.66453 \\ \mathrm{H} & 7.12009 & 1.19669 & -1.5759 \\ \mathrm{H} & 8.18083 & -1.66375 & -1.0628 \\ \mathrm{H} & 8.3987 & -0.29994 & 0.06586 \\ \mathrm{H} & 7.67123 & -1.80919 & 0.637 \\ \mathrm{H} & 3.87314 & -0.14106 & -0.05749 \\ \mathrm{H} & 4.49765 & 1.47494 & 2.31935 \\ \mathrm{H} & 1.34189 & -0.74911 & 4.16887 \\ \mathrm{H} & 1.21166 & 0.77799 & 5.09655 \\ \mathrm{H} & 0.22886 & 0.54016 & 3.62026 \\ \mathrm{H} & 2.26987 & 2.23612 & 0.35352 \\ \mathrm{H} & 2.74014 & 3.09732 & 1.81395 \\ \mathrm{H} & 2.92292 & 3.14963 & -1.65614 \\ \mathrm{H} & 4.45329 & 4.52024 & -3.0408 \\ \mathrm{H} & 6.50468 & 5.50033 & -2.02893 \\ \mathrm{H} & 7.00453 & 5.09212 & 0.37315 \\ \mathrm{H} & 5.48369 & 3.72167 & 1.74515\end{array}$

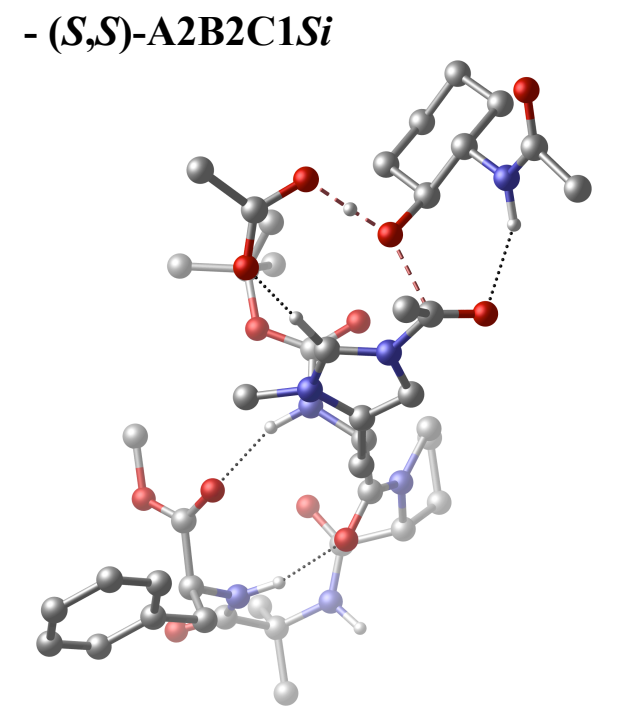

$\begin{array}{lccc}\mathrm{N} & 0.9433 & -0.85708 & 0.42553 \\ \mathrm{C} & 0.88534 & -1.17866 & -0.99047 \\ \mathrm{C} & 0.46582 & 0.05203 & -1.82948 \\ \mathrm{C} & 2.27471 & -1.65394 & -1.46667 \\ \mathrm{C} & -0.93099 & 0.52999 & -1.58068 \\ \mathrm{O} & 3.11156 & -0.82849 & -1.86444 \\ \mathrm{~N} & -1.30784 & 1.4695 & -0.61063 \\ \mathrm{C} & -2.08262 & 0.20382 & -2.24103 \\ \mathrm{C} & -2.62715 & 1.68913 & -0.69546 \\ \mathrm{~N} & -3.11054 & 0.93489 & -1.68548 \\ \mathrm{~N} & 2.55756 & -2.97298 & -1.41991 \\ \mathrm{C} & 1.71825 & -4.07776 & -0.90886 \\ \mathrm{C} & 3.91171 & -3.41893 & -1.78194 \\ \mathrm{C} & 2.75521 & -5.13453 & -0.51729 \\ \mathrm{C} & 3.85644 & -4.95034 & -1.57312 \\ \mathrm{C} & -0.46351 & 2.11837 & 0.39721 \\ \mathrm{C} & 7.17028 & -1.54584 & -0.8848 \\ \mathrm{~N} & 6.03212 & -2.23909 & -1.50773 \\ \mathrm{C} & 6.78444 & -0.17265 & -0.26161 \\ \mathrm{O} & 7.60463 & 0.42226 & 0.42901 \\ \mathrm{C} & 8.18122 & -1.24012 & -2.00762 \\ \mathrm{C} & 7.83231 & -2.41911 & 0.1943 \\ \mathrm{C} & 0.25356 & -1.56509 & 1.36925 \\ \mathrm{O} & -0.4438 & -2.54212 & 1.13037 \\ \mathrm{O} & 0.45246 & -1.0043 & 2.58641 \\ \mathrm{C} & -0.16604 & -1.58496 & 3.80004 \\ \mathrm{C} & 0.35079 & -3.01014 & 4.01555 \\ \mathrm{C} & 0.33248 & -0.6522 & 4.90555 \\ \mathrm{C} & -1.6916 & -1.52547 & 3.68765 \\ \mathrm{C} & 2.84533 & 5.80811 & -0.44737 \\ \mathrm{C} & 3.12942 & 4.50853 & -0.87045 \\ \mathrm{C} & 3.86729 & 6.62454 & 0.03718 \\ \mathrm{C} & 4.43451 & 4.00393 & -0.8216 \\ \mathrm{C} & 5.17404 & 6.1349 & 0.08999 \\ \mathrm{C} & 4.74657 & 2.59075 & -1.27089 \\ \mathrm{C} & 5.45353 & 4.8375 & -0.33737\end{array}$




\begin{tabular}{|c|c|c|c|}
\hline & 5.16057 & 171 & \\
\hline & 5.57188 & 0.35119 & -0.60256 \\
\hline & 4.02213 & 1.46084 & \\
\hline & 2.84013 & 1.37318 & \\
\hline & 4.47941 & 1.34639 & \\
\hline & 3.50146 & 1.08042 & 3.16106 \\
\hline & & -2.74229 & \\
\hline & & -2.75513 & -0.8554 \\
\hline & & -1.21384 & 1.47046 \\
\hline & & -2.61536 & \\
\hline & -5.64079 & -0.82861 & 0.03105 \\
\hline & -7.25987 & -2.75023 & 1.63806 \\
\hline & -7.1 & -0.9 & -0. \\
\hline & -7.6 & -2.3975 & \\
\hline & -5.1 & 0.47522 & -0.2 \\
\hline & -7.4 & -0.6 & -1 \\
\hline & -8.7 & -0.3 & \\
\hline & -9.7 & -0.3 & -1 \\
\hline & -8.8 & -0.0 & -3 \\
\hline & -4.5 & 0.82 & -2 \\
\hline & -5.2 & 2.15 & -2 \\
\hline & -4.7 & -0.1 & 173 \\
\hline & -5.30689 & 3.16204 & \\
\hline & -4.20 & 3.42509 & 0.98764 \\
\hline & -6.0 & 2.14608 & 1.25 \\
\hline & -5.87237 & 4.10 & 2.57122 \\
\hline & -0.08181 & -0.96233 & 6894 \\
\hline & 1.42 & -0.68 & \\
\hline & 0.02 & & \\
\hline & 1.4 & -3.0 & 023 \\
\hline & -0.00 & -3.3 & 033 \\
\hline & -0.0 & -3.6 & 3.22975 \\
\hline & -2.0 & -0.49996 & 3.49716 \\
\hline & -2.05246 & -2.1 & 2.8 \\
\hline & -2.13998 & -1.85386 & 3057 \\
\hline & 1.5 & -0.1 & 0.72178 \\
\hline & 0.14137 & -1.9 & -1.09859 \\
\hline & 1.19 & 0.8 & \\
\hline & 0.54 & -0.21012 & -2.8 \\
\hline & -0.27076 & 1.43523 & 1.22524 \\
\hline & -0.99462 & 2.99795 & 0.7616 \\
\hline & 0.48915 & 2.4146 & -0.0413 \\
\hline & -3.22448 & 2.3406 & -0.03518 \\
\hline & -2.27014 & -0.47457 & -3.0568 \\
\hline & -6.31992 & 1.97289 & -2.2641 \\
\hline & -4.93762 & 2.61763 & -3.18113 \\
\hline & -5.01582 & 2.82798 & -1.41 \\
\hline & -6.05744 & 3.56015 & 3.50026 \\
\hline & -6.83828 & 4.49534 & 2.23271 \\
\hline & -5.18682 & 4.93574 & 2.75632 \\
\hline & -5.59377 & 1.30026 & 0.44659 \\
\hline & -5.12979 & -1.51093 & -0.66914 \\
\hline & -4.16907 & -1.13661 & 1.56731 \\
\hline & -5.69085 & -0.46559 & 2.14683 \\
\hline
\end{tabular}

$\begin{array}{llll}\mathrm{H} & -5.48836 & -2.82526 & 2.90492 \\ \mathrm{H} & -5.22407 & -3.36745 & 1.25204 \\ \mathrm{H} & -7.59443 & -3.76705 & 1.87612 \\ \mathrm{H} & -7.78948 & -2.07787 & 2.32714 \\ \mathrm{H} & -8.71696 & -2.44651 & 0.0428 \\ \mathrm{H} & -7.17699 & -3.12246 & -0.4945 \\ \mathrm{H} & -7.68368 & -0.25232 & 0.43352 \\ \mathrm{H} & -6.71202 & -0.64165 & -2.25115 \\ \mathrm{H} & -7.93073 & -0.04384 & -4.02256 \\ \mathrm{H} & -9.38815 & 0.89021 & -3.59139 \\ \mathrm{H} & -9.52846 & -0.82716 & -3.95185 \\ \mathrm{H} & 1.092 & -3.75455 & -0.07742 \\ \mathrm{H} & 1.0628 & -4.44802 & -1.70948 \\ \mathrm{H} & 2.33527 & -6.14342 & -0.50734 \\ \mathrm{H} & 3.15442 & -4.90584 & 0.47402 \\ \mathrm{H} & 4.82229 & -5.35695 & -1.26402 \\ \mathrm{H} & 3.57411 & -5.43509 & -2.51403 \\ \mathrm{H} & 4.11555 & -3.15524 & -2.82568 \\ \mathrm{H} & 5.97537 & -2.1926 & -2.51484 \\ \mathrm{H} & 8.21486 & -3.33768 & -0.26111 \\ \mathrm{H} & 8.65796 & -1.86522 & 0.64267 \\ \mathrm{H} & 7.11518 & -2.68047 & 0.97221 \\ \mathrm{H} & 9.02273 & -0.68345 & -1.59277 \\ \mathrm{H} & 8.55344 & -2.17015 & -2.44889 \\ \mathrm{H} & 7.72677 & -0.63079 & -2.79769 \\ \mathrm{H} & 4.88908 & -0.19328 & -1.12319 \\ \mathrm{H} & 6.02013 & 2.06502 & 0.42464 \\ \mathrm{H} & 3.88431 & 2.1508 & -1.7805 \\ \mathrm{H} & 5.57946 & 2.59513 & -1.98292 \\ \mathrm{H} & 2.98185 & 0.14064 & 2.965 \\ \mathrm{H} & 2.7722 & 1.89244 & 3.20712 \\ \mathrm{H} & 4.06744 & 1.02038 & 4.08938 \\ \mathrm{H} & 6.47587 & 4.46867 & -0.30015 \\ \mathrm{H} & 5.97727 & 6.76547 & 0.46008 \\ \mathrm{H} & 3.64905 & 7.63584 & 0.36723 \\ \mathrm{H} & 1.82686 & 6.18259 & -0.49803 \\ \mathrm{H} & 2.3314 & 3.87734 & -1.25194\end{array}$

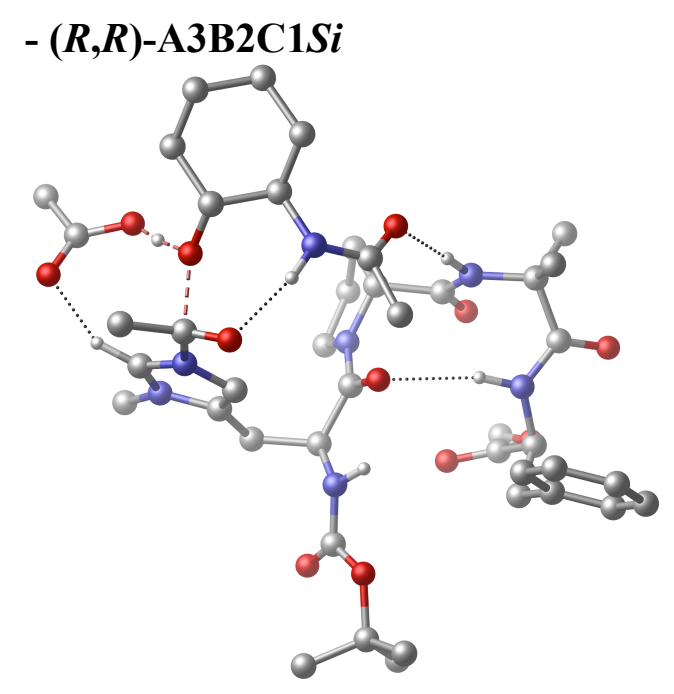




\begin{tabular}{|c|c|c|c|}
\hline N & 1.26471 & 2.47208 & 0.0726 \\
\hline C & 0.61272 & 2.07063 & 1.30273 \\
\hline $\mathrm{C}$ & -0.66756 & 2.90492 & 1.5432 \\
\hline $\mathrm{C}$ & 0.3904 & 0.54594 & 1.21026 \\
\hline $\mathrm{C}$ & -1.81631 & 2.6412 & 0.61745 \\
\hline $\mathrm{O}$ & 0.65021 & -0.04052 & 0.14701 \\
\hline $\mathrm{N}$ & -3.08622 & 3.16359 & 0.89128 \\
\hline $\mathrm{C}$ & -1.94953 & 1.9443 & -0.55179 \\
\hline C & -3.9377 & 2.78553 & -0.07084 \\
\hline $\mathrm{N}$ & -3.26663 & 2.05186 & -0.95729 \\
\hline $\mathrm{N}$ & -0.10001 & -0.11508 & 2.27803 \\
\hline C & -0.2699 & 0.39548 & 3.65127 \\
\hline $\mathrm{C}$ & -0.36551 & -1.57123 & 2.18191 \\
\hline C & -0.45176 & -0.87344 & 4.49722 \\
\hline C & -1.0835 & -1.86899 & 3.51168 \\
\hline C & -3.47065 & 3.97194 & 2.04491 \\
\hline $\mathrm{C}$ & 2.27951 & -3.94956 & 0.7182 \\
\hline $\mathrm{N}$ & 1.08793 & -3.12721 & 0.98034 \\
\hline $\mathrm{C}$ & 3.57586 & -3.09258 & 0.76446 \\
\hline $\mathrm{O}$ & 4.65095 & -3.59661 & 1.05756 \\
\hline C & 2.15413 & -4.51656 & -0.70935 \\
\hline $\mathrm{C}$ & 2.39423 & -5.09013 & 1.74183 \\
\hline $\mathrm{C}$ & 2.03457 & 3.60026 & 0.03756 \\
\hline $\mathrm{O}$ & 2.00235 & 4.4727 & 0.89602 \\
\hline $\mathrm{O}$ & 2.78576 & 3.59985 & -1.08613 \\
\hline $\mathrm{C}$ & 3.67108 & 4.73071 & -1.41471 \\
\hline $\mathrm{C}$ & 4.75118 & 4.87676 & -0.33855 \\
\hline $\mathrm{C}$ & 4.283 & 4.29403 & -2.74772 \\
\hline $\mathrm{C}$ & 2.8444 & 6.00911 & -1.58601 \\
\hline C & 6.49527 & -1.60095 & -3.4005 \\
\hline $\mathrm{C}$ & 6.23819 & -0.99841 & -2.16968 \\
\hline C & 5.49268 & -1.67086 & -4.36988 \\
\hline $\mathrm{C}$ & 4.97726 & -0.4586 & -1.88168 \\
\hline C & 4.23394 & -1.13495 & -4.09738 \\
\hline $\mathrm{C}$ & 4.69733 & 0.18123 & -0.53988 \\
\hline C & 3.98098 & -0.53508 & -2.86254 \\
\hline C & 4.52583 & -0.83543 & 0.60528 \\
\hline $\mathrm{N}$ & 3.44858 & -1.78643 & 0.35873 \\
\hline $\mathrm{C}$ & 4.19852 & -0.12113 & 1.92285 \\
\hline $\mathrm{O}$ & 3.38334 & 0.77745 & 2.03434 \\
\hline $\mathrm{O}$ & 4.87917 & -0.64562 & 2.94881 \\
\hline $\mathrm{C}$ & 4.47534 & -0.20533 & 4.25786 \\
\hline $\mathrm{O}$ & 1.83139 & -2.23039 & 2.94796 \\
\hline $\mathrm{C}$ & 0.96326 & -2.34839 & 2.07691 \\
\hline C & -3.847 & $1.329-2$ & 2.15418 \\
\hline $\mathrm{C}$ & -5.13754 & 1.94122 & -2.65326 \\
\hline $\mathrm{O}$ & -3.02092 & 0.81076 & -2.89662 \\
\hline $\mathrm{C}$ & -5.64745 & -4.1616 & -1.46117 \\
\hline C & -6.72138 & -3.10891 & -1.76309 \\
\hline $\mathrm{C}$ & -4.27768 & -3.69744 & -1.96806 \\
\hline $\mathrm{C}$ & -6.34076 & -1.75163 & -1.15664 \\
\hline $\mathrm{C}$ & -3.87312 & -2.32654 & -1.38758 \\
\hline $\mathrm{C}$ & -4.96157 & -1.25512 & -1.62603 \\
\hline $\mathrm{O}$ & -4.61397 & -0.03353 & -1.00067 \\
\hline
\end{tabular}

\begin{tabular}{|c|c|c|}
\hline $\mathrm{N}$ & $-2.62394-1.87002$ & -1.99484 \\
\hline $\mathrm{C}$ & $-1.40502-2.31898$ & -1.6489 \\
\hline $\mathrm{O}$ & $-1.22824-3.16412$ & -0.75037 \\
\hline $\mathrm{C}$ & $-6.69918 \quad 1.35361$ & 1.1595 \\
\hline $\mathrm{C}$ & $-7.68324 \quad 1.17006$ & 2.30311 \\
\hline $\mathrm{O}$ & -6.761192 .35952 & 0.43229 \\
\hline $\mathrm{O}$ & $\begin{array}{ll}-5.8371 & 0.38754\end{array}$ & 1.04661 \\
\hline $\mathrm{C}$ & $-0.24701 \quad-1.76068$ & -2.45512 \\
\hline $\mathrm{H}$ & $4.82028 \quad 3.3482$ & -2.63556 \\
\hline $\mathrm{H}$ & $4.98559 \quad 5.05283$ & -3.10461 \\
\hline $\mathrm{H}$ & $3.50501 \quad 4.15886$ & -3.50446 \\
\hline $\mathrm{H}$ & $5.28771 \quad 3.9318$ & -0.20992 \\
\hline $\mathrm{H}$ & $5.47361 \quad 5.63993$ & -0.64538 \\
\hline $\mathrm{H}$ & $4.31402 \quad 5.16732$ & 0.61688 \\
\hline $\mathrm{H}$ & $3.49 \quad 6.81877-$ & 1.94099 \\
\hline $\mathrm{H}$ & $2.38981 \quad 6.31034$ & -0.64184 \\
\hline $\mathrm{H}$ & $2.05525 \quad 5.85466$ & -2.32869 \\
\hline $\mathrm{H}$ & $1.47098 \quad 1.71279$ & -0.56486 \\
\hline $\mathrm{H}$ & $1.28407 \quad 2.26589$ & 2.14683 \\
\hline $\mathrm{H}$ & $-1.00395 \quad 2.76172$ & 2.57487 \\
\hline $\mathrm{H}$ & $-0.34968 \quad 3.95205$ & 1.47192 \\
\hline $\mathrm{H}$ & $-1.23855 \quad 1.37202$ & -1.12119 \\
\hline $\mathrm{H}$ & $-4.54071 \quad 4.16957$ & 1.98264 \\
\hline $\mathrm{H}$ & $-3.26289 \quad 3.43075$ & 2.97065 \\
\hline $\mathrm{H}$ & $-2.92271 \quad 4.91705$ & 2.04787 \\
\hline $\mathrm{H}$ & $\begin{array}{lll}-5.01788 & 2.92938\end{array}$ & -0.0468 \\
\hline $\mathrm{H}$ & -5.859692 .14622 & -1.86314 \\
\hline $\mathrm{H}$ & $\begin{array}{ll}-4.89911 & 2.86397\end{array}$ & -3.19547 \\
\hline $\mathrm{H}$ & $-5.57545 \quad 1.23954$ & -3.36464 \\
\hline $\mathrm{H}$ & $\begin{array}{ll}-8.22825 & 2.09527\end{array}$ & 2.49309 \\
\hline $\mathrm{H}$ & $\begin{array}{ll}-8.39681 & 0.38287\end{array}$ & 2.03727 \\
\hline $\mathrm{H}$ & $-7.16199 \quad 0.83823$ & 3.20506 \\
\hline $\mathrm{H}$ & $\begin{array}{lll}-5.26256 & 0.25281\end{array}$ & 0.03245 \\
\hline $\mathrm{H}$ & $-6.32871-1.82805$ & -0.06302 \\
\hline $\mathrm{H}$ & $\begin{array}{ll}-7.0873 & -0.98897\end{array}$ & -1.40636 \\
\hline $\mathrm{H}$ & $-7.69624-3.4281$ & -1.37589 \\
\hline $\mathrm{H}$ & $\begin{array}{ll}-6.83601 & -3.01077\end{array}$ & -2.85204 \\
\hline $\mathrm{H}$ & $-5.60104-4.33385$ & -0.37679 \\
\hline $\mathrm{H}$ & $-5.90878-5.12294$ & -1.91868 \\
\hline $\mathrm{H}$ & $\begin{array}{ll}-4.3019 & -3.61459\end{array}$ & -3.06355 \\
\hline $\mathrm{H}$ & $-3.49606-4.4221$ & -1.72014 \\
\hline $\mathrm{H}$ & $-3.71746-2.42553$ & -0.30615 \\
\hline $\mathrm{H}$ & $-5.01147-1.08903$ & -2.71762 \\
\hline $\mathrm{H}$ & $-2.6806-1.05725$ & -2.60335 \\
\hline $\mathrm{H}$ & $0.50487-1.36996$ & -1.76898 \\
\hline $\mathrm{H}$ & $\begin{array}{ll}-0.5527 & -0.97003\end{array}$ & -3.14462 \\
\hline $\mathrm{H}$ & $0.20118-2.57718$ & -3.02987 \\
\hline $\mathrm{H}$ & $0.59835 \quad 0.98694$ & 3.95491 \\
\hline $\mathrm{H}$ & $\begin{array}{ll}-1.16498 & 1.02657\end{array}$ & 3.71691 \\
\hline $\mathrm{H}$ & $-1.07145-0.68805$ & 5.37872 \\
\hline $\mathrm{H}$ & $0.52223-1.24884$ & 4.81398 \\
\hline $\mathrm{H}$ & $\begin{array}{lll}-0.95727 & -2.90905\end{array}$ & 3.82024 \\
\hline $\mathrm{H}$ & $-2.15581-1.67766$ & 3.3948 \\
\hline $\mathrm{H}$ & $\begin{array}{lll}-0.99159 & -1.7717\end{array}$ & 1.31057 \\
\hline
\end{tabular}




$\begin{array}{llll}\mathrm{H} & 0.31163 & -3.16684 & 0.30901 \\ \mathrm{H} & 2.12426 & -3.71587 & -1.4534 \\ \mathrm{H} & 1.24168 & -5.11313 & -0.80311 \\ \mathrm{H} & 3.01618 & -5.15233 & -0.92357 \\ \mathrm{H} & 1.52824 & -5.75292 & 1.65025 \\ \mathrm{H} & 3.30825 & -5.658 & 1.56289 \\ \mathrm{H} & 2.43326 & -4.686 & 2.75462 \\ \mathrm{H} & 2.51408 & -1.40202 & 0.2691 \\ \mathrm{H} & 5.42953 & -1.43157 & 0.74092 \\ \mathrm{H} & 3.45144 & -0.53457 & 4.44738 \\ \mathrm{H} & 5.16575 & -0.68313 & 4.95204 \\ \mathrm{H} & 4.53798 & 0.88252 & 4.33684 \\ \mathrm{H} & 3.80345 & 0.80916 & -0.59272 \\ \mathrm{H} & 5.52339 & 0.84916 & -0.2639 \\ \mathrm{H} & 2.99895 & -0.11638 & -2.65786 \\ \mathrm{H} & 3.44796 & -1.1809 & -4.84603 \\ \mathrm{H} & 5.69256 & -2.13741 & -5.33013 \\ \mathrm{H} & 7.47928 & -2.01369 & -3.60394 \\ \mathrm{H} & 7.02592 & -0.94703 & -1.42178\end{array}$

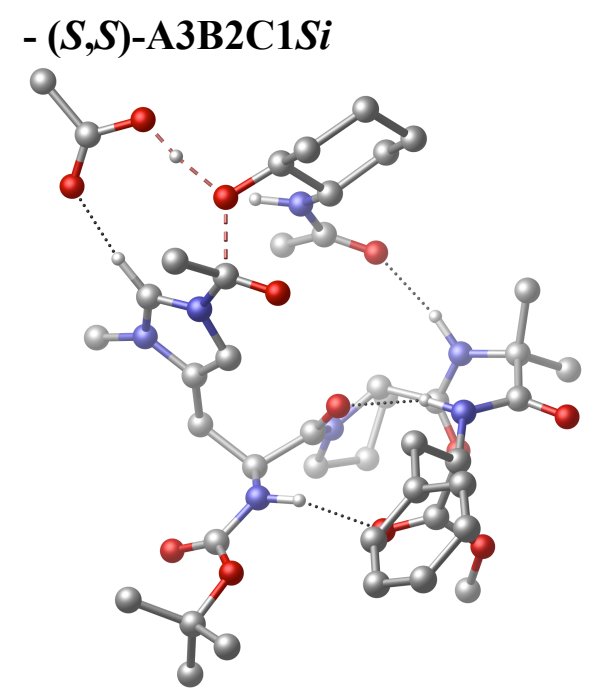

$\begin{array}{lccc}\mathrm{N} & -1.71128 & 2.01411 & -1.34767 \\ \mathrm{C} & -1.09731 & 1.33666 & -2.47517 \\ \mathrm{C} & 0.3064 & 1.90492 & -2.83275 \\ \mathrm{C} & -1.00827 & -0.1673 & -2.17878 \\ \mathrm{C} & 1.34597 & 1.88896 & -1.75079 \\ \mathrm{O} & -0.72409 & -0.56437 & -1.04372 \\ \mathrm{~N} & 2.62004 & 2.42934 & -1.97139 \\ \mathrm{C} & 1.35002 & 1.46381 & -0.4512 \\ \mathrm{C} & 3.34772 & 2.32201 & -0.84588 \\ \mathrm{~N} & 2.58835 & 1.74465 & 0.08221 \\ \mathrm{~N} & -1.15057 & -1.02239 & -3.22055 \\ \mathrm{C} & -1.78934 & -0.75442 & -4.52461 \\ \mathrm{C} & -0.81681 & -2.4515 & -3.03872 \\ \mathrm{C} & -2.2041 & -2.15172 & -5.00107 \\ \mathrm{C} & -1.10319 & -3.05619 & -4.43037 \\ \mathrm{C} & 3.10919 & 3.02936 & -3.20847 \\ \mathrm{C} & -1.52846 & -4.54445 & 0.0971\end{array}$

\begin{tabular}{|c|c|c|c|}
\hline & 0.95609 & -3.7795 & -1.0203 \\
\hline & -2.38506 & -3.65675 & 1.04243 \\
\hline & -3.21192 & -4.1688 & 1.78709 \\
\hline & -0.3516 & -5.08532 & 0.9322 \\
\hline & -2.39517 & -5.70416 & -0.4197 \\
\hline & -2.15241 & 3.29626 & -1.51839 \\
\hline & -1.90022 & 3.9759 & -2.50898 \\
\hline & -2.86753 & 3.69317 & -0.44422 \\
\hline & -3.44714 & 5.04627 & -0.3783 \\
\hline & -4.45159 & 5.24342 & -1.5183 \\
\hline & -4.16151 & 5.0382 & 0.9748 \\
\hline & -2.33077 & 6.09566 & -0.39507 \\
\hline & -4.32642 & 2.45797 & 3.97541 \\
\hline & -3.51694 & 1.72539 & 3.10522 \\
\hline & -4.61952 & 1.96631 & 5.24788 \\
\hline & -2.98594 & 0.48848 & 3.4951 \\
\hline & -4.09639 & 0.73514 & 5.6469 \\
\hline & -2.12865 & -0.3348 & 2.5551 \\
\hline & -3.28744 & 0.00533 & 4.77645 \\
\hline & -2.94144 & -1.37858 & 1.76949 \\
\hline & -2.08499 & -2.32227 & 1.0573 \\
\hline & -3.84811 & -0.74854 & 0.70687 \\
\hline & -3.59433 & 0.26256 & 0.07198 \\
\hline & -4.92652 & -1.50401 & 0.48498 \\
\hline & -5.70042 & -1.18016 & -0.68476 \\
\hline & -2.91223 & -3.04586 & -1.97962 \\
\hline & -1.68252 & -3.1103 & -1.9484 \\
\hline & 4.55418 & -0.99455 & 3.1083 \\
\hline & 4.75795 & -2.43258 & 3.61304 \\
\hline & 4.59836 & -0.93898 & 1.57256 \\
\hline & 3.7472 & -3.40017 & 2.97817 \\
\hline & 3.55071 & -1.89679 & 0.95868 \\
\hline & 3.79023 & -3.33489 & 1.44386 \\
\hline & 4.49243 & 0.3596 & 1.01624 \\
\hline & 3.67026 & -1.75647 & -0.49692 \\
\hline & 2.83973 & -2.29586 & -1.40923 \\
\hline & 1.94664 & -3.10999 & -1.13786 \\
\hline & 3.07757 & -1.85202 & -2.84792 \\
\hline & 2.94766 & 1.39675 & 1.54148 \\
\hline & 3.70864 & 2.5268 & 2.205 \\
\hline & 2.10443 & 0.7197 & 2.10407 \\
\hline & 7.08526 & 2.24395 & 0.17993 \\
\hline & 6.26056 & 2.87956 & -0.48425 \\
\hline & 6.80911 & 1.22945 & 0.9592 \\
\hline & 8.55848 & 2.59863 & 0.16275 \\
\hline & -4.64808 & 6.00297 & 1.14633 \\
\hline & -4.92342 & 4.25411 & 1.00244 \\
\hline & -3.45049 & 4.85898 & 1.78614 \\
\hline & -5.20605 & 4.45093 & -1.49769 \\
\hline & -4.96335 & 6.20304 & -1.39306 \\
\hline & -3.95236 & 5.23374 & -2.48718 \\
\hline & -1.6103 & 5.89453 & 0.40386 \\
\hline & -1.80904 & 6.0974 & -1.35222 \\
\hline & -2.75832 & $7.0888 \mathrm{~S}$ & -0.2239 \\
\hline
\end{tabular}




\begin{tabular}{|c|c|c|c|}
\hline $\mathrm{H}$ & -2.16617 & 1.43959 & -0.63916 \\
\hline $\mathrm{H}$ & -1.69939 & 1.51232 & -3.37282 \\
\hline $\mathrm{H}$ & 0.67496 & 1.35734 & -3.71152 \\
\hline $\mathrm{H}$ & 0.13027 & 2.93728 & -3.15393 \\
\hline $\mathrm{H}$ & 3.07319 & 2.30318 & -4.02438 \\
\hline $\mathrm{H}$ & 4.14133 & 3.34462 & -3.0546 \\
\hline $\mathrm{H}$ & 2.50171 & 3.89771 & -3.47496 \\
\hline $\mathrm{H}$ & 4.39596 & 2.60119 & -0.7334 \\
\hline $\mathrm{H}$ & 0.59635 & 0.96138 & 0.12689 \\
\hline $\mathrm{H}$ & 4.11727 & 2.15528 & 3.14484 \\
\hline $\mathrm{H}$ & 2.99679 & 3.32994 & 2.42846 \\
\hline $\mathrm{H}$ & 4.51703 & 2.92358 & 1.59088 \\
\hline $\mathrm{H}$ & 9.14224 & 1.73961 & -0.18143 \\
\hline $\mathrm{H}$ & 8.89425 & 2.8279 & 1.17841 \\
\hline $\mathrm{H}$ & 8.73363 & 3.45414 & -0.48922 \\
\hline $\mathrm{H}$ & 5.77383 & 0.9347 & 0.96022 \\
\hline $\mathrm{H}$ & 5.58781 & -1.32689 & 1.26371 \\
\hline $\mathrm{H}$ & 5.33856 & -0.3364 & 3.50089 \\
\hline $\mathrm{H}$ & 3.59302 & -0.60399 & 3.4554 \\
\hline $\mathrm{H}$ & 4.67902 & -2.46259 & 4.7062 \\
\hline $\mathrm{H}$ & 5.77635 & -2.76555 & 3.36572 \\
\hline $\mathrm{H}$ & 3.93996 & -4.42663 & 3.31175 \\
\hline $\mathrm{H}$ & 2.73566 & -3.14515 & 3.32325 \\
\hline $\mathrm{H}$ & 3.04404 & -3.99857 & 0.99945 \\
\hline $\mathrm{H}$ & 4.77392 & -3.669 & 1.08467 \\
\hline $\mathrm{H}$ & 2.54993 & -1.56966 & 1.2631 \\
\hline $\mathrm{H}$ & 4.2217 & -0.95156 & -0.76995 \\
\hline $\mathrm{H}$ & 3.99524 & -1.27253 & -2.97786 \\
\hline $\mathrm{H}$ & 2.22745 & -1.2475 & -3.18023 \\
\hline $\mathrm{H}$ & 3.12367 & -2.73797 & -3.48593 \\
\hline $\mathrm{H}$ & -2.64463 & -0.08462 & -4.4117 \\
\hline $\mathrm{H}$ & -1.07717 & -0.29058 & -5.2212 \\
\hline $\mathrm{H}$ & -2.29344 & -2.20792 & -6.08916 \\
\hline $\mathrm{H}$ & -3.16128 & -2.41957 & -4.54778 \\
\hline $\mathrm{H}$ & -1.40259 & -4.10456 & -4.36626 \\
\hline $\mathrm{H}$ & -0.19855 & -3.00187 & -5.04667 \\
\hline $\mathrm{H}$ & 0.23495 & -2.5428 & -2.75989 \\
\hline $\mathrm{H}$ & 0.05447 & -3.65045 & -1.03527 \\
\hline $\mathrm{H}$ & -1.77243 & -6.39938 & -0.99131 \\
\hline $\mathrm{H}$ & -2.84488 & -6.22747 & 0.42485 \\
\hline $\mathrm{H}$ & -3.19335 & -5.32699 & -1.06052 \\
\hline $\mathrm{H}$ & -0.73947 & -5.61996 & 1.80174 \\
\hline $\mathrm{H}$ & 0.2526 & -5.77474 & 0.3341 \\
\hline $\mathrm{H}$ & 0.29289 & -4.27193 & 1.2785 \\
\hline $\mathrm{H}$ & -1.47594 & -1.93913 & 0.34032 \\
\hline $\mathrm{H}$ & -3.55922 & -1.97662 & 2.44216 \\
\hline $\mathrm{H}$ & -1.59973 & 0.31257 & 1.84948 \\
\hline $\mathrm{H}$ & -1.36845 & -0.88048 & 3.1223 \\
\hline $\mathrm{H}$ & -5.11318 & -1.40855 & -1.57726 \\
\hline $\mathrm{H}$ & -5.98449 & -0.12583 & -0.68075 \\
\hline $\mathrm{H}$ & -6.58143 & -1.81895 & -0.63588 \\
\hline $\mathrm{H}$ & -2.87835 & -0.95063 & 5.09493 \\
\hline $\mathrm{H}$ & -4.31469 & 0.3445 & 6.63687 \\
\hline $\mathrm{H}$ & -5.24801 & 2.53826 & 5.92461 \\
\hline
\end{tabular}

$\begin{array}{llll}\mathrm{H} & -4.72801 & 3.41615 & 3.65778 \\ \mathrm{H} & -3.29711 & 2.11215 & 2.1145\end{array}$

\section{- $(R, R)-\mathrm{A} 1 \mathrm{~B} 1 \mathrm{C} 2 S i$}

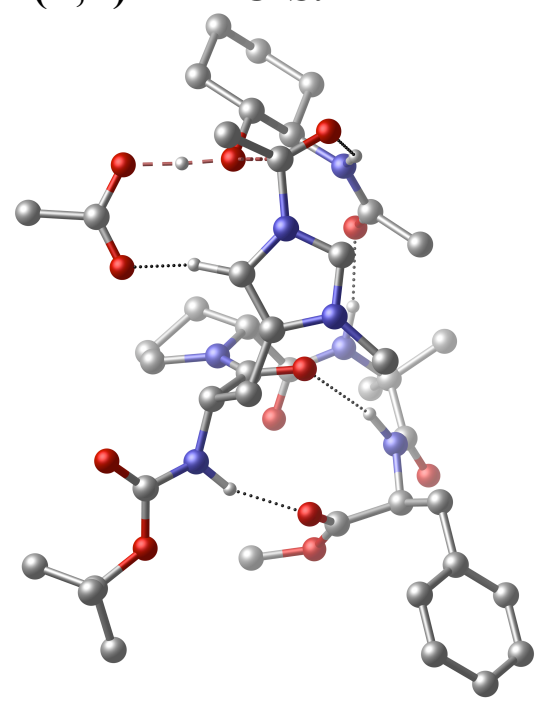

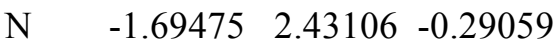

$\begin{array}{llll}\text { C } & -0.41525 & 1.98781 & 0.23749\end{array}$

$\begin{array}{llll}\text { C } & -0.44257 & 1.92522 & 1.78362\end{array}$

$\begin{array}{llll}\text { C } & -0.07503 & 0.60334 & -0.34983\end{array}$

$\begin{array}{llll}\text { C } & 0.80156 & 1.33253 & 2.36824\end{array}$

$\begin{array}{llll}\mathrm{O} & -0.25501 & -0.43645 & 0.31414\end{array}$

$\begin{array}{llll}\mathrm{N} & 0.78625 & 0.24453 & 3.24137\end{array}$

$\begin{array}{llll}\mathrm{C} & 2.11974 & 1.62356 & 2.13832\end{array}$

$\begin{array}{llll}\text { C } & 2.04645 & -0.10783 & 3.51391\end{array}$

$\begin{array}{llll}\mathrm{N} & 2.87142 & 0.71188 & 2.85752\end{array}$

$\begin{array}{llll}\mathrm{N} & 0.43169 & 0.58469 & -1.59318\end{array}$

$\begin{array}{llll}\mathrm{C} & 0.62706 & 1.74464 & -2.48955\end{array}$

$\begin{array}{llll}\text { C } & 0.89636 & -0.67838 & -2.18732\end{array}$

$\begin{array}{llll}\text { C } & 0.82464 & 1.08417 & -3.85792\end{array}$

$\begin{array}{llll}\text { C } & 1.54544 & -0.22911 & -3.51629\end{array}$

$\begin{array}{llll}\text { C } & -0.40123 & -0.48406 & 3.67937\end{array}$

$\begin{array}{lllll}\mathrm{C} & -1.02235 & -3.99871 & -2.12156\end{array}$

$\mathrm{N} \quad-0.07432-2.89242 \quad-1.94573$

$\begin{array}{llll}\text { C } & -2.41232 & -3.68162 & -1.50418\end{array}$

$\begin{array}{llll}\text { O } & -3.40614 & -4.29739 & -1.86698\end{array}$

$\begin{array}{llll}\text { C } & -0.4619 & -5.21562 & -1.35932\end{array}$

$\begin{array}{llll}\text { C } & -1.2047 & -4.33982 & -3.61043\end{array}$

$\begin{array}{lllll}\text { C } & -1.99143 & 3.76535 & -0.36602\end{array}$

$\begin{array}{lllll}\mathrm{O} & -1.19465 & 4.66478 & -0.14545\end{array}$

$\begin{array}{lllll}\text { O } & -3.28848 & 3.9144 & -0.73838\end{array}$

$\begin{array}{llll}\text { C } & -3.87137 & 5.25315 & -0.91981\end{array}$

$\begin{array}{llll}\text { C } & -3.1425 & 5.99272 & -2.04642\end{array}$

$\begin{array}{llll}C & -5.31406 & 4.93646 & -1.3211\end{array}$

$\begin{array}{llll}\text { C } & -3.8339 & 6.02775 & 0.40201\end{array}$

$\begin{array}{llll}\text { C } & -6.88297 & -0.50763 & 2.91701\end{array}$

$\begin{array}{llll}\text { C } & -5.59137 & -0.7613 & 2.45285\end{array}$

$\begin{array}{llll}\text { C } & -7.80925 & -1.54616 & 3.01932\end{array}$ 


\begin{tabular}{|c|c|c|c|}
\hline & -5.20336 & -2.05695 & \\
\hline & -7.43575 & -2.84054 & \\
\hline & 3.81099 & -2.34222 & \\
\hline & & -3.09126 & \\
\hline & 3.71314 & -2.27317 & \\
\hline & -2.43448 & -2.77543 & -0.4726 \\
\hline & -3.83812 & & \\
\hline & -3.4309 & 0.13983 & \\
\hline & -4.35351 & -0.81904 & \\
\hline & -4.23094 & & -2.4 \\
\hline & -1.31 & & -2.9 \\
\hline & -0.2 & & -2.4 \\
\hline$C$ & 4.3 & & \\
\hline C & 5.0 & & 3.22 \\
\hline $\mathrm{O}$ & 4.7 & & 3.3 \\
\hline & 6.9 & & -1.4 \\
\hline $\mathrm{C}$ & 7.6 & & -0.8 \\
\hline $\mathrm{C}$ & 5.9 & & -0.4 \\
\hline C & 6.6 & & -0.4 \\
\hline $\mathrm{C}$ & 4.9 & & \\
\hline $\mathrm{C}$ & & & \\
\hline $\mathrm{O}$ & & & \\
\hline $\mathrm{N}$ & 3.9 & & \\
\hline C & & & \\
\hline $\mathrm{O}$ & & & -0.6 \\
\hline $\mathrm{C}$ & & & \\
\hline $\mathrm{C}$ & & & \\
\hline $\mathrm{O}$ & & & -0. \\
\hline $\mathrm{O}$ & & & \\
\hline C & & -3.2 & \\
\hline $\mathrm{H}$ & & & -2.2 \\
\hline $\mathrm{H}$ & & & -1. \\
\hline $\mathrm{H}$ & & & -0.5 \\
\hline $\mathrm{H}$ & & & -2.5 \\
\hline $\mathrm{H}$ & & & -2.2 \\
\hline $\mathrm{H}$ & & & -1.7 \\
\hline $\mathrm{H}$ & 99 & & 0.2 \\
\hline $\mathrm{H}$ & & 6.2 & 0.7 \\
\hline $\mathrm{H}$ & & & 1.1 \\
\hline $\mathrm{H}$ & & & -0.2 \\
\hline $\mathrm{H}$ & & & -0.0 \\
\hline $\mathrm{H}$ & & & 2.14 \\
\hline $\mathrm{H}$ & & & 2.08 \\
\hline $\mathrm{H}$ & & 2.31 & 1.414 \\
\hline $\mathrm{H}$ & -0.92078 & -0.85454 & 2.7936 \\
\hline $\mathrm{H}$ & & -1.31975 & 4.3064 \\
\hline $\mathrm{H}$ & -1.05864 & 0.17093 & 4.2544 \\
\hline $\mathrm{H}$ & 2.36571 & -0.90728 & 4.1603 \\
\hline $\mathrm{H}$ & & 2.625 & 2.54102 \\
\hline $\mathrm{H}$ & 4.90114 & 2.14202 & 4.2603 \\
\hline $\mathrm{H}$ & 6.16761 & 1.63116 & 3.12722 \\
\hline $\mathrm{H}$ & 4.97797 & 4.12743 & -2.3001 \\
\hline $\mathrm{H}$ & 3.60618 & 5.11327 & -1.7217 \\
\hline & 5.19372 & 5.16216 & -0.8946 \\
\hline
\end{tabular}

$\begin{array}{llll}\mathrm{H} & 4.77521 & 1.55366 & 0.51311 \\ \mathrm{H} & 6.09101 & 0.53358 & -1.28382 \\ \mathrm{H} & 7.11503 & 1.001 & 0.05571 \\ \mathrm{H} & 8.3468 & -0.47053 & -1.56877 \\ \mathrm{H} & 8.25004 & -1.22538 & 0.01607 \\ \mathrm{H} & 6.45366 & -1.85259 & -2.38777 \\ \mathrm{H} & 7.69723 & -2.90516 & -1.72581 \\ \mathrm{H} & 6.44187 & -3.11972 & 0.4084 \\ \mathrm{H} & 5.39321 & -3.57127 & -0.93599 \\ \mathrm{H} & 4.32266 & -1.35101 & -0.91078 \\ \mathrm{H} & 6.11977 & -0.77658 & 1.48115 \\ \mathrm{H} & 4.09437 & -1.98669 & 1.90211 \\ \mathrm{H} & 2.29235 & -3.15631 & 2.66325 \\ \mathrm{H} & 1.56893 & -4.30858 & 1.51664 \\ \mathrm{H} & 0.97398 & -2.64324 & 1.56789 \\ \mathrm{H} & -0.24703 & 2.39732 & -2.45879 \\ \mathrm{H} & 1.5054 & 2.3151 & -2.16662 \\ \mathrm{H} & 1.39247 & 1.71863 & -4.54363 \\ \mathrm{H} & -0.14706 & 0.86026 & -4.3062 \\ \mathrm{H} & 1.44989 & -0.98783 & -4.29677 \\ \mathrm{H} & 2.61309 & -0.05051 & -3.35018 \\ \mathrm{H} & 1.62417 & -1.14772 & -1.5231 \\ \mathrm{H} & 0.80612 & -3.08223 & -1.46165 \\ \mathrm{H} & -0.34289 & -4.99385 & -0.29418 \\ \mathrm{H} & 0.51384 & -5.50106 & -1.76419 \\ \mathrm{H} & -1.14849 & -6.05844 & -1.4632 \\ \mathrm{H} & -0.24839 & -4.66823 & -4.02889 \\ \mathrm{H} & -1.94315 & -5.13506 & -3.72046 \\ \mathrm{H} & -1.55347 & -3.46298 & -4.15777 \\ \mathrm{H} & -1.61485 & -2.19518 & -0.31714 \\ \mathrm{H} & -4.48127 & -2.89495 & -0.43896 \\ \mathrm{H} & -3.19223 & 0.52512 & -2.78851 \\ \mathrm{H} & -4.88125 & 0.297 & -3.33597 \\ \mathrm{H} & -4.54119 & 1.26684 & -1.86328 \\ \mathrm{H} & -3.49813 & -3.34939 & 1.85279 \\ \mathrm{H} & -3.09351 & -1.6331 & 1.98508 \\ \mathrm{H} & -5.85868 & -4.10241 & 1.91089 \\ \mathrm{H} & -8.14908 & -3.65616 & 2.73034 \\ \mathrm{H} & -8.81402 & -1.3493 & 3.38178 \\ \mathrm{H} & -7.16525 & 0.503 & 3.19875 \\ \mathrm{H} & -4.87746 & 0.05168 & 2.3599\end{array}$




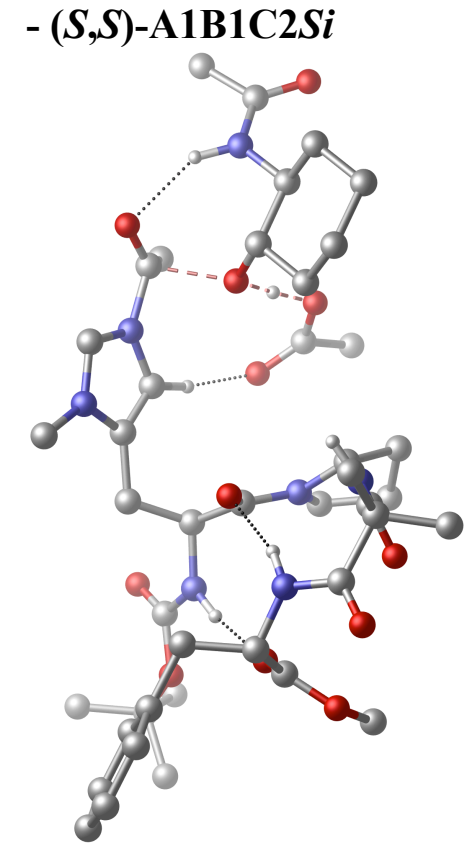

$\mathrm{N} \quad \begin{array}{llll}-2.35905 & -1.91311 & 0.47057\end{array}$

$\begin{array}{lllll}\text { C } & -0.9662 & -1.75377 & 0.08907\end{array}$

$\begin{array}{lllll}\text { C } & -0.74423 & -2.13139 & -1.39047\end{array}$

$\begin{array}{llll}\text { C } & -0.53579 & -0.29165 & 0.32373\end{array}$

C $\quad 0.68789-2.02576 \quad-1.81314$

$\begin{array}{lllll}\mathrm{O} & -0.56699 & 0.52937 & -0.61079\end{array}$

$\mathrm{N} \quad 1.06251 \quad-1.69786 \quad-3.11934$

C $\quad 1.85221 \quad-2.19078-1.11243$

$\begin{array}{llll}\mathrm{C} & 2.4003 & -1.66678 & -3.19137\end{array}$

$\mathrm{N} \quad 2.89448 \quad-1.9662 \quad-1.99281$

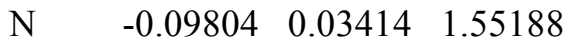

$\begin{array}{lllll}\text { C } & -0.1205 & -0.8199 & 2.76071\end{array}$

$\begin{array}{llll}\text { C } & 0.5643 & 1.33081 & 1.78134\end{array}$

$\begin{array}{lllll}\text { C } & 0.18309 & 0.17942 & 3.88341\end{array}$

$\begin{array}{llll}\text { C } & 1.12443 & 1.19347 & 3.21366\end{array}$

$\begin{array}{llll}\text { C } & 0.15689 & -1.37984 & -4.21945\end{array}$

$\begin{array}{llll}\mathrm{C} & -0.91738 & 4.56762 & 0.30676\end{array}$

$\begin{array}{llll}\mathrm{N} & -0.11211 & 3.39138 & 0.66908\end{array}$

C $\quad-2.37155 \quad 4.17678 \quad-0.08687$

$\begin{array}{lllll}\text { O } & -3.27165 & 5.00321 & -0.02689\end{array}$

$\begin{array}{lllll}\text { C } & -0.26611 & 5.20904 & -0.93291\end{array}$

$\begin{array}{lllll}\text { C } & -0.96495 & 5.57183 & 1.46901\end{array}$

$\begin{array}{llll}\text { C } & -2.8808 & -3.16316 & 0.64537\end{array}$

$\begin{array}{lllll}\mathrm{O} & -2.23287 & -4.19483 & 0.52316\end{array}$

$\begin{array}{llll}\mathrm{O} & -4.19527 & -3.06964 & 0.95466\end{array}$

$\begin{array}{llll}\text { C } & -4.99673 & -4.27261 & 1.23493\end{array}$

$\begin{array}{llll}\text { C } & -4.42548 & -5.01234 & 2.449\end{array}$

$\begin{array}{lllll}\text { C } & -6.37282 & -3.68511 & 1.55638\end{array}$

$\begin{array}{lllll}\text { C } & -5.05939 & -5.16292 & -0.01017\end{array}$

$\begin{array}{lllll}\text { C } & -7.10773 & -0.78212 & -2.02448\end{array}$

C $\quad-5.81086-0.34353-1.75116$

$\begin{array}{lllll}\text { C } & -7.97928 & 0.01446 & -2.76769\end{array}$

$\begin{array}{llll}\text { C } & -5.3638 & 0.899 & -2.22101\end{array}$ $\begin{array}{llll}\text { C } & -7.54589 & 1.2554 & -3.23804\end{array}$

$\begin{array}{lllll}\text { C } & -3.96274 & 1.3962 & -1.92749\end{array}$

$\begin{array}{lllll}\text { C } & -6.24926 & 1.69054 & -2.96652\end{array}$

$\begin{array}{lllll}\mathrm{C} & -3.88962 & 2.35454 & -0.727\end{array}$

$\begin{array}{lllll}\mathrm{N} & -2.54643 & 2.91068 & -0.57952\end{array}$

$\begin{array}{lllll}\text { C } & -4.20795 & 1.65189 & 0.59864\end{array}$

$\begin{array}{llll}\mathrm{O} & -3.88201 & 0.50846 & 0.87346\end{array}$

$\begin{array}{lllll}\mathrm{O} & -4.80322 & 2.47278 & 1.46089\end{array}$

$\begin{array}{llll}\text { C } & -4.9048 & 1.99572 & 2.81906\end{array}$

$\begin{array}{llll}\text { O } & -1.40126 & 2.61331 & 2.39205\end{array}$

$\begin{array}{lllll}\text { C } & -0.42714 & 2.50134 & 1.65164\end{array}$

$\begin{array}{lllll}\text { C } & 4.04293 & 1.92306 & -0.30748\end{array}$

$\begin{array}{llll}\text { C } & 4.68327 & 3.27215 & -0.66175\end{array}$

$\begin{array}{llll}\text { C } & 4.86102 & 0.718 & -0.80603\end{array}$

$\begin{array}{lllll}\text { C } & 6.12712 & 3.34018 & -0.14773\end{array}$

$\begin{array}{lllll}\text { C } & 6.32279 & 0.79958 & -0.31697\end{array}$

$\begin{array}{llll}\text { C } & 6.95163 & 2.16178 & -0.67904\end{array}$

O $\quad 4.20929-0.4792 \quad-0.42865$

N $\quad \begin{array}{llll}7.09332 & -0.30315 & -0.88722\end{array}$

$\begin{array}{lllll}\text { C } & 8.30075 & -0.68249 & -0.38478\end{array}$

$\begin{array}{lllll}\text { O } & 8.82875 & -0.13526 & 0.57995\end{array}$

C $\quad 8.96954-1.84709-1.10651$

C $\quad 4.38055-1.97423 \quad-1.73696$

C $\quad 4.81073 \quad-3.02742 \quad-0.74865$

$\begin{array}{lllll}\text { O } & 5.05558 & -1.58016 & -2.68037\end{array}$

$\begin{array}{llll}\text { C } & 3.50792 & -1.75819 & 2.46472\end{array}$

$\begin{array}{llll}\mathrm{O} & 2.6967 & -2.46785 & 1.8324\end{array}$

$\begin{array}{llll}\text { O } & 4.22346 & -0.8081 & 1.96915\end{array}$

C $\quad 3.71513-1.99337 \quad 3.95626$

$\mathrm{H} \quad-7.08015 \quad-4.48744 \quad 1.78585$

$\begin{array}{llll}\mathrm{H} & -6.31379 & -3.01582 & 2.41912\end{array}$

$\mathrm{H} \quad-6.75871 \quad-3.11421 \quad 0.70684$

$\mathrm{H} \quad-4.35091 \quad-4.33453 \quad 3.30507$

$\mathrm{H} \quad \begin{array}{llll}-5.09275 & -5.83504 & 2.72547\end{array}$

$\mathrm{H} \quad-3.43737 \quad-5.41806 \quad 2.23134$

$\mathrm{H} \quad-5.43328 \quad-4.59287 \quad-0.86671$

$\mathrm{H} \quad-4.0748 \quad-5.5633 \quad-0.2524$

$\mathrm{H} \quad \begin{array}{llll}-5.74629 & -5.99659 & 0.16797\end{array}$

$\mathrm{H} \quad-2.96369-1.09832 \quad 0.55898$

$\mathrm{H} \quad-0.36354 \quad-2.42263 \quad 0.71163$

$\mathrm{H} \quad-1.10834 \quad-3.1568 \quad-1.51592$

$\mathrm{H} \quad-1.36065-1.46894-2.00222$

$\mathrm{H} \quad-0.51663 \quad-2.21798 \quad-4.40894$

$\mathrm{H} \quad 0.74694 \quad-1.18522 \quad-5.11517$

$\mathrm{H} \quad-0.42708 \quad-0.49095 \quad-3.97096$

$\mathrm{H} \quad 2.99993 \quad-1.43608 \quad-4.05613$

$\mathrm{H} \quad 2.04283 \quad-2.37229 \quad-0.05631$

$\mathrm{H} \quad 5.81227 \quad-2.77423 \quad-0.40199$

$\mathrm{H} \quad 4.85211 \quad-3.98656 \quad-1.27876$

$\mathrm{H} \quad 4.14151 \quad-3.11277 \quad 0.10749$

$\mathrm{H} \quad 3.4874 \quad-1.08132 \quad 4.51741$

$\mathrm{H} \quad 4.76796 \quad-2.22349 \quad 4.14554$

H $\quad 3.08892 \quad-2.8134 \quad 4.3099$

$\begin{array}{llll}\mathrm{H} & 4.19683 & -0.66788 & 0.71197\end{array}$ 


$\begin{array}{cccc}\mathrm{H} & 4.88067 & 0.73574 & -1.9087 \\ \mathrm{H} & 3.03301 & 1.83911 & -0.72905 \\ \mathrm{H} & 3.94581 & 1.83587 & 0.78275 \\ \mathrm{H} & 4.08471 & 4.09298 & -0.247 \\ \mathrm{H} & 4.68058 & 3.40442 & -1.75323 \\ \mathrm{H} & 6.59366 & 4.28836 & -0.43949 \\ \mathrm{H} & 6.12324 & 3.31902 & 0.95078 \\ \mathrm{H} & 7.96943 & 2.18129 & -0.28065 \\ \mathrm{H} & 7.02954 & 2.23036 & -1.77359 \\ \mathrm{H} & 6.33682 & 0.68439 & 0.774 \\ \mathrm{H} & 6.70352 & -0.78339 & -1.69082 \\ \mathrm{H} & 8.36856 & -2.26466 & -1.91899 \\ \mathrm{H} & 9.18589 & -2.63276 & -0.37746 \\ \mathrm{H} & 9.92792 & -1.50807 & -1.51016 \\ \mathrm{H} & -1.09908 & -1.29349 & 2.86584 \\ \mathrm{H} & 0.65465 & -1.59075 & 2.68436 \\ \mathrm{H} & 0.63124 & -0.30806 & 4.75286 \\ \mathrm{H} & -0.73525 & 0.68423 & 4.19348 \\ \mathrm{H} & 1.1536 & 2.15305 & 3.73458 \\ \mathrm{H} & 2.14441 & 0.80149 & 3.15348 \\ \mathrm{H} & 1.36858 & 1.43902 & 1.04755 \\ \mathrm{H} & 0.62428 & 3.12281 & 0.0323 \\ \mathrm{H} & 0.04502 & 5.93463 & 1.68221 \\ \mathrm{H} & -1.60633 & 6.41196 & 1.20044 \\ \mathrm{H} & -1.37044 & 5.09689 & 2.36313 \\ \mathrm{H} & -0.8576 & 6.07077 & -1.24872 \\ \mathrm{H} & 0.74835 & 5.54942 & -0.70033 \\ \mathrm{H} & -0.21979 & 4.50196 & -1.76839 \\ \mathrm{H} & -1.78502 & 2.23897 & -0.51512 \\ \mathrm{H} & -4.57072 & 3.1984 & -0.84853 \\ \mathrm{H} & -3.28874 & 0.55507 & -1.73798 \\ \mathrm{H} & -3.56897 & 1.9412 & -2.79206 \\ \mathrm{H} & -3.90169 & 1.86159 & 3.22856 \\ \mathrm{H} & -5.45209 & 1.05148 & 2.85287 \\ \mathrm{H} & -5.44198 & 2.77482 & 3.35792 \\ \mathrm{H} & -5.91606 & 2.65536 & -3.34208 \\ \mathrm{H} & -8.2155 & 1.88259 & -3.8196 \\ \mathrm{H} & -8.98743 & -0.32888 & -2.98079 \\ \mathrm{H} & -7.43639 & -1.75046 & -1.65718 \\ \mathrm{H} & -5.14491 & -0.96796 & -1.16284 \\ & & & \end{array}$

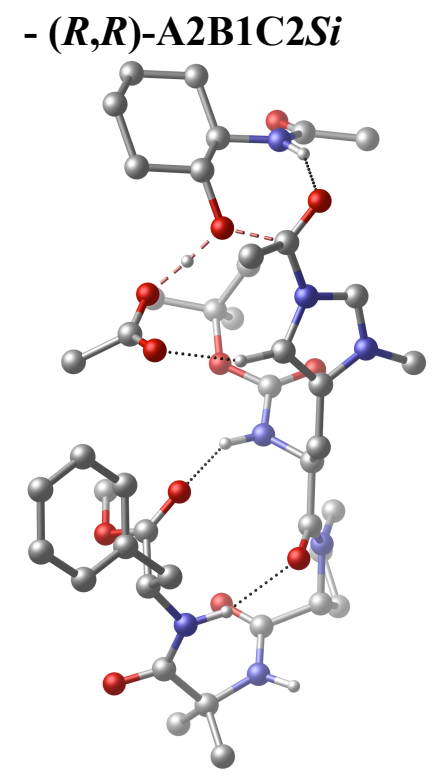

$\begin{array}{llll}\mathrm{N} & 0.69555 & -1.63305 & 0.10718 \\ \mathrm{C} & 1.18334 & -1.89309 & -1.23332 \\ \mathrm{C} & 0.67401 & -0.84722 & -2.25342 \\ \mathrm{C} & 2.72554 & -1.93981 & -1.2539 \\ \mathrm{C} & -0.81578 & -0.69945 & -2.32602 \\ \mathrm{O} & 3.39926 & -1.01572 & -1.72827 \\ \mathrm{~N} & -1.68517 & -1.68089 & -2.8203 \\ \mathrm{C} & -1.60068 & 0.38318 & -2.02766 \\ \mathrm{C} & -2.92965 & -1.19305 & -2.81696 \\ \mathrm{~N} & -2.90565 & 0.0538 & -2.34878 \\ \mathrm{~N} & 3.31198 & -3.05205 & -0.75126 \\ \mathrm{C} & 2.6642 & -4.20941 & -0.10538 \\ \mathrm{C} & 4.77814 & -3.14875 & -0.74387 \\ \mathrm{C} & 3.80535 & -4.8293 & 0.70736 \\ \mathrm{C} & 5.04185 & -4.55723 & -0.16334 \\ \mathrm{C} & -1.34326 & -3.05385 & -3.19067 \\ \mathrm{C} & 7.1437 & -0.2487 & 0.19729 \\ \mathrm{~N} & 6.40023 & -1.35022 & -0.43484 \\ \mathrm{C} & 6.22976 & 0.95889 & 0.55553 \\ \mathrm{O} & 6.62912 & 1.80523 & 1.34577 \\ \mathrm{C} & 8.16713 & 0.26203 & -0.83469 \\ \mathrm{C} & 7.86347 & -0.73141 & 1.46659 \\ \mathrm{C} & -0.39133 & -2.32531 & 0.58276 \\ \mathrm{O} & -0.82809 & -3.34183 & 0.0433 \\ \mathrm{O} & -0.84476 & -1.75365 & 1.7022 \\ \mathrm{C} & -2.06393 & -2.24849 & 2.395 \\ \mathrm{C} & -1.766 & -3.62455 & 2.99355 \\ \mathrm{C} & -2.26763 & -1.19058 & 3.48008 \\ \mathrm{C} & -3.25064 & -2.26916 & 1.43143 \\ \mathrm{C} & 0.81286 & 5.34241 & -0.46634 \\ \mathrm{C} & 1.49708 & 4.15193 & -0.71852 \\ \mathrm{C} & 1.51482 & 6.54141 & -0.33879 \\ \mathrm{C} & 2.89146 & 4.14049 & -0.84864 \\ \mathrm{C} & 2.90603 & 6.54252 & -0.46253 \\ \mathrm{C} & 3.64749 & 2.85411 & -1.11264\end{array}$ 


\begin{tabular}{|c|c|c|c|}
\hline & 3.58645 & & \\
\hline & 4.10146 & 2.13033 & \\
\hline & 5.05386 & & \\
\hline & .94695 & & \\
\hline & 1.96894 & & \\
\hline & 3.18941 & 1.42232 & \\
\hline & 2.29524 & 0.617 & 3.03201 \\
\hline & 4.98638 & -1.86321 & \\
\hline & & -2.0 & \\
\hline & 23 & & \\
\hline & & 2.3 & \\
\hline & & 0.3 & \\
\hline & 68 & & \\
\hline & -7.0 & & \\
\hline & -7.7 & 0.417 & \\
\hline & -5.6 & 2.45821 & \\
\hline & -6.3663 & 0.045 & $0.6^{2}$ \\
\hline C & -5.6 & 1.2 & \\
\hline $\mathrm{O}$ & -4.3 & 0.9 & \\
\hline & -6.3 & -1.0 & \\
\hline & -6.5 & -2.3 & \\
\hline & & -2.7 & \\
\hline & & & \\
\hline & & & \\
\hline 0 & & & -0. \\
\hline & -2.6 & 1.5 & \\
\hline 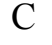 & & -3.3 & $5-1$. \\
\hline & 7 & -1.1 & \\
\hline & & -1.4 & 24. \\
\hline & 19 & -0.2 & \\
\hline & 45 & -3.5 & 07 \\
\hline & -2.6 & -3.96229 & \\
\hline 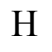 & -1.5 & -4.35 & 2.2 \\
\hline t. & & -2.4 & 41. \\
\hline 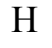 & & -3. & 0 . \\
\hline 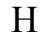 & & -1 & \\
\hline 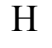 & & -0. & 92 \\
\hline 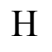 & & -2.8 & -1 \\
\hline F & & & -1 \\
\hline 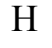 & & -1 & $3-3$ \\
\hline 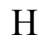 & -1.3 & 1.33 & $9-1.5$ \\
\hline $\mathrm{H}$ & & -3.6 & -2.28 \\
\hline $\mathrm{H}$ & & -3.4 & $3-3.75$ \\
\hline $\mathrm{H}$ & & -3.05158 & $\begin{array}{ll}8 & -3.82\end{array}$ \\
\hline $\mathrm{H}$ & & -1.69739 & $9-3.1491$ \\
\hline $\mathrm{H}$ & & 2.7386 & -1.9005 \\
\hline $\mathrm{H}$ & -3.60968 & 2.52165 & $5-3.6080$ \\
\hline $\mathrm{H}$ & & 2.90862 & -2.37314 \\
\hline $\mathrm{H}$ & & 2.34696 & 2.90491 \\
\hline $\mathrm{H}$ & & 3.37469 & 91.7790 \\
\hline $\mathrm{H}$ & -1.54571 & 3.94294 & 2.4296 \\
\hline $\mathrm{H}$ & -3.49708 & 1.26538 & 0.3097 \\
\hline $\mathrm{H}$ & -5.02445 & 2.21631 & 1.8555 \\
\hline & -5.17371 & 3.31141 & $\begin{array}{ll}1 & 0.4917\end{array}$ \\
\hline
\end{tabular}

$\begin{array}{llll}\mathrm{H} & -7.02774 & 3.65471 & 2.1582 \\ \mathrm{H} & -7.65857 & 3.1685 & 0.58995 \\ \mathrm{H} & -7.24228 & 1.32485 & 3.00322 \\ \mathrm{H} & -8.79354 & 1.86698 & 2.37636 \\ \mathrm{H} & -8.39337 & 0.6638 & 0.23333 \\ \mathrm{H} & -8.23536 & -0.46482 & 1.58012 \\ \mathrm{H} & -5.78941 & -0.30126 & 1.50973 \\ \mathrm{H} & -6.21687 & 1.53761 & -0.87697 \\ \mathrm{H} & -6.13867 & -0.7984 & -1.29022 \\ \mathrm{H} & -6.38167 & -2.87425 & -2.11453 \\ \mathrm{H} & -7.40765 & -3.93991 & -1.12531 \\ \mathrm{H} & -5.65764 & -4.02034 & -0.95138 \\ \mathrm{H} & 1.82048 & -3.89542 & 0.50702 \\ \mathrm{H} & 2.2932 & -4.91025 & -0.86641 \\ \mathrm{H} & 3.64482 & -5.89205 & 0.90548 \\ \mathrm{H} & 3.90475 & -4.30063 & 1.65841 \\ \mathrm{H} & 5.97907 & -4.60159 & 0.39628 \\ \mathrm{H} & 5.10533 & -5.28164 & -0.98284 \\ \mathrm{H} & 5.15276 & -3.05122 & -1.76891 \\ \mathrm{H} & 6.55183 & -1.49782 & -1.42212 \\ \mathrm{H} & 7.67206 & 0.60023 & -1.75188 \\ \mathrm{H} & 8.88288 & -0.52727 & -1.08734 \\ \mathrm{H} & 8.71347 & 1.1073 & -0.41264 \\ \mathrm{H} & 8.59606 & -1.50073 & 1.20356 \\ \mathrm{H} & 8.37198 & 0.11222 & 1.93432 \\ \mathrm{H} & 7.14802 & -1.14588 & 2.17732 \\ \mathrm{H} & 4.72247 & 0.29568 & -0.70721 \\ \mathrm{H} & 4.61519 & 2.81633 & 0.8441 \\ \mathrm{H} & 2.4261 & -0.43709 & 2.77492 \\ \mathrm{H} & 2.58899 & 0.79273 & 4.06576 \\ \mathrm{H} & 1.25764 & 0.91317 & 2.86791 \\ \mathrm{H} & 4.55538 & 3.06914 & -1.6856 \\ \mathrm{H} & 3.04269 & 2.16289 & -1.70814 \\ \mathrm{H} & 4.66948 & 5.36093 & -0.8166 \\ \mathrm{H} & 3.46202 & 7.47113 & -0.36667 \\ \mathrm{H} & 0.98424 & 7.46973 & -0.14601 \\ \mathrm{H} & -0.26778 & 5.30605 & -0.3731 \\ \mathrm{H} & 0.92474 & 3.23431 & -0.80254 \\ & & & \\ & & & \end{array}$




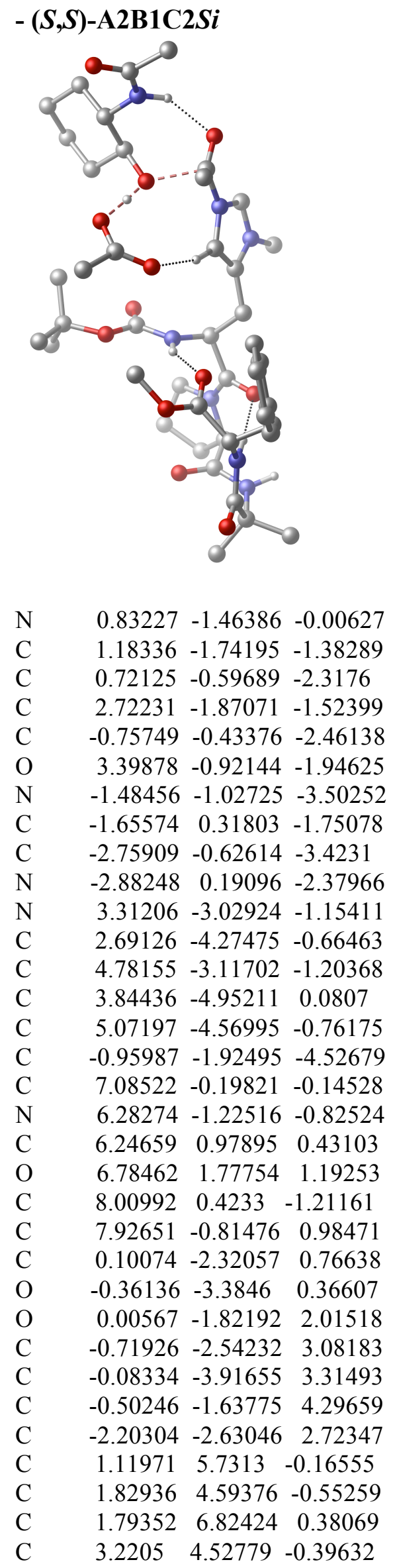

\begin{tabular}{|c|c|c|c|}
\hline $\mathrm{C}$ & 3.17963 & 6.77144 & 0.53942 \\
\hline $\mathrm{C}$ & 3.98886 & 3.28204 & -0.78135 \\
\hline $\mathrm{C}$ & 3.88531 & 5.63165 & 0.15484 \\
\hline $\mathrm{C}$ & 4.17499 & 2.27785 & 0.38917 \\
\hline $\mathrm{N}$ & 4.97045 & 1.12851 & -0.02208 \\
\hline $\mathrm{C}$ & 2.81609 & 1.79372 & 0.87965 \\
\hline $\mathrm{O}$ & 2.09324 & 1.07439 & 0.20481 \\
\hline $\mathrm{O}$ & 2.50027 & 2.25045 & 2.08327 \\
\hline $\mathrm{C}$ & 1.19272 & 1.8729 & 2.58823 \\
\hline $\mathrm{O}$ & 5.18284 & -2.13937 & 0.9689 \\
\hline $\mathrm{C}$ & 5.41901 & -2.0983 & -0.23247 \\
\hline $\mathrm{C}$ & -5.25292 & -2.20238 & 0.64404 \\
\hline $\mathrm{C}$ & -6.45901 & -3.13382 & 0.82426 \\
\hline $\mathrm{C}$ & -5.56362 & -0.98489 & -0.24198 \\
\hline $\mathrm{C}$ & -7.66432 & -2.36011 & 1.37354 \\
\hline $\mathrm{C}$ & -6.79328 & -0.21592 & 0.28782 \\
\hline $\mathrm{C}$ & -7.99688 & -1.16527 & 0.47409 \\
\hline $\mathrm{O}$ & -4.39766 & -0.18386 & -0.36413 \\
\hline $\mathrm{N}$ & -7.13315 & 0.87502 & -0.62468 \\
\hline $\mathrm{C}$ & -7.95806 & 1.89748 & -0.26612 \\
\hline $\mathrm{O}$ & -8.46472 & 1.99452 & 0.84856 \\
\hline $\mathrm{C}$ & -8.21901 & 2.93501 & -1.35295 \\
\hline $\mathrm{C}$ & -4.20048 & 0.86247 & -2.08411 \\
\hline $\mathrm{C}$ & -4.04766 & 2.22081 & -1.45478 \\
\hline $\mathrm{O}$ & -5.11717 & 0.51758 & -2.81591 \\
\hline $\mathrm{C}$ & -2.67519 & 1.52091 & 1.88165 \\
\hline $\mathrm{O}$ & -1.85945 & 1.79388 & 0.96898 \\
\hline $\mathrm{O}$ & -3.68907 & 0.74304 & 1.78531 \\
\hline $\mathrm{C}$ & -2.49673 & 2.15633 & 3.2599 \\
\hline $\mathrm{H}$ & -1.00929 & -2.05813 & 5.16999 \\
\hline $\mathrm{H}$ & 0.56314 & -1.54473 & 4.52669 \\
\hline $\mathrm{H}$ & -0.90909 & -0.64046 & 4.10932 \\
\hline $\mathrm{H}$ & 0.99086 & -3.81329 & 3.49976 \\
\hline $\mathrm{H}$ & -0.53522 & -4.37943 & 4.1981 \\
\hline $\mathrm{H}$ & -0.23629 & -4.5723 & 2.45788 \\
\hline $\mathrm{H}$ & -2.6084 & -1.6348 & 2.52144 \\
\hline $\mathrm{H}$ & -2.35237 & -3.26125 & 1.84635 \\
\hline $\mathrm{H}$ & -2.75683 & -3.06359 & 3.56319 \\
\hline $\mathrm{H}$ & 1.15346 & -0.58543 & 0.39251 \\
\hline $\mathrm{H}$ & 0.69542 & -2.68646 & -1.64095 \\
\hline $\mathrm{H}$ & 1.14773 & 0.32845 & -1.92678 \\
\hline $\mathrm{H}$ & 1.17697 & -0.73996 & -3.30222 \\
\hline $\mathrm{H}$ & -0.19574 & -1.41862 & -5.12072 \\
\hline $\mathrm{H}$ & -1.7778 & -2.22678 & -5.18104 \\
\hline $\mathrm{H}$ & -0.52912 & -2.81438 & -4.06255 \\
\hline $\mathrm{H}$ & -3.56763 & -0.89229 & -4.0836 \\
\hline $\mathrm{H}$ & -1.54837 & 0.88494 & -0.82547 \\
\hline $\mathrm{H}$ & -5.0059 & 2.49762 & -1.01723 \\
\hline $\mathrm{H}$ & -3.80654 & 2.93104 & -2.25561 \\
\hline $\mathrm{H}$ & -3.27309 & 2.25494 & -0.6873 \\
\hline $\mathrm{H}$ & -2.26348 & 1.38219 & 3.99889 \\
\hline $\mathrm{H}$ & -3.43768 & 2.61674 & 3.57425 \\
\hline $\mathrm{H}$ & -1.70296 & 2.90489 & 3.25249 \\
\hline $\mathrm{H}$ & -4.05432 & 0.2499 & 0.58127 \\
\hline
\end{tabular}




$\begin{array}{llll}\mathrm{H} & -5.80331 & -1.33497 & -1.25945 \\ \mathrm{H} & -4.39993 & -2.73442 & 0.20918 \\ \mathrm{H} & -4.92801 & -1.83152 & 1.62365 \\ \mathrm{H} & -6.1918 & -3.96337 & 1.48989 \\ \mathrm{H} & -6.72714 & -3.58389 & -0.14267 \\ \mathrm{H} & -8.53738 & -3.01739 & 1.46308 \\ \mathrm{H} & -7.43632 & -2.00309 & 2.38728 \\ \mathrm{H} & -8.8268 & -0.58175 & 0.88131 \\ \mathrm{H} & -8.30882 & -1.52665 & -0.51658 \\ \mathrm{H} & -6.53799 & 0.22858 & 1.25774 \\ \mathrm{H} & -6.75437 & 0.83714 & -1.56407 \\ \mathrm{H} & -7.67253 & 2.74763 & -2.28148 \\ \mathrm{H} & -7.94649 & 3.92134 & -0.96749 \\ \mathrm{H} & -9.29111 & 2.95735 & -1.56851 \\ \mathrm{H} & 1.8198 & -4.07829 & -0.04241 \\ \mathrm{H} & 2.36404 & -4.88831 & -1.51708 \\ \mathrm{H} & 3.70326 & -6.03238 & 0.16968 \\ \mathrm{H} & 3.9397 & -4.52009 & 1.07973 \\ \mathrm{H} & 6.01007 & -4.64823 & -0.20825 \\ \mathrm{H} & 5.14712 & -5.21144 & -1.64681 \\ \mathrm{H} & 5.12267 & -2.92564 & -2.22695 \\ \mathrm{H} & 6.23194 & -1.16552 & -1.83209 \\ \mathrm{H} & 8.61345 & -1.55761 & 0.5677 \\ \mathrm{H} & 8.49744 & -0.0254 & 1.47471 \\ \mathrm{H} & 7.28571 & -1.29807 & 1.72203 \\ \mathrm{H} & 8.60372 & 1.21735 & -0.75642 \\ \mathrm{H} & 8.6839 & -0.33597 & -1.62062 \\ \mathrm{H} & 7.42962 & 0.86003 & -2.03294 \\ \mathrm{H} & 4.54056 & 0.43928 & -0.63221 \\ \mathrm{H} & 4.70925 & 2.75834 & 1.20902 \\ \mathrm{H} & 3.48416 & 2.75628 & -1.59922 \\ \mathrm{H} & 4.99285 & 3.54602 & -1.12865 \\ \mathrm{H} & 1.17126 & 0.79952 & 2.78962 \\ \mathrm{H} & 0.40504 & 2.12007 & 1.87283 \\ \mathrm{H} & 1.07693 & 2.43583 & 3.51301 \\ \mathrm{H} & 4.96484 & 5.59663 & 0.28083 \\ \mathrm{H} & 3.71235 & 7.61873 & 0.96166 \\ \mathrm{H} & 1.24306 & 7.71142 & 0.67978 \\ \mathrm{H} & 0.04134 & 5.76149 & -0.29111 \\ \mathrm{H} & 1.29752 & 3.7451 & -0.97473\end{array}$

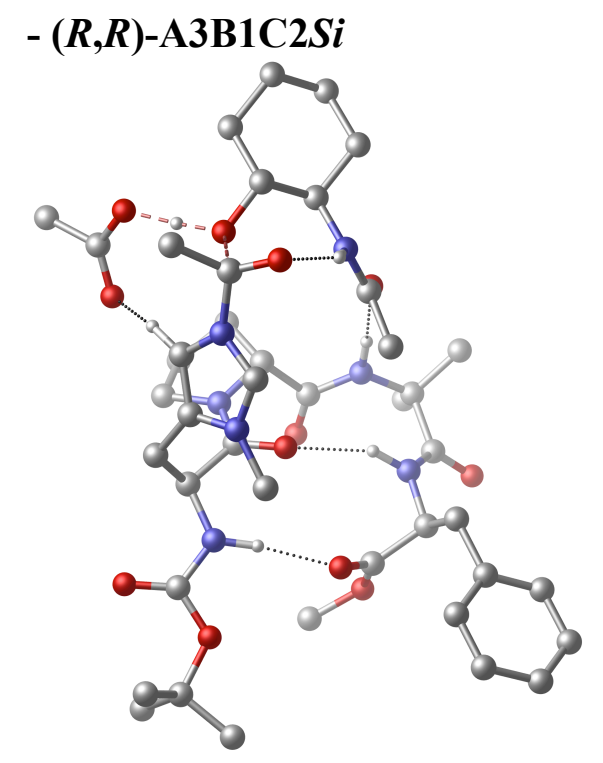

$\begin{array}{lccc}\mathrm{N} & 2.0964 & 2.46016 & 0.44463 \\ \mathrm{C} & 0.73053 & 2.51198 & 0.93599 \\ \mathrm{C} & -0.23378 & 3.29863 & -0.0146 \\ \mathrm{C} & 0.21469 & 1.08541 & 1.15086 \\ \mathrm{C} & -0.95838 & 2.53357 & -1.0782 \\ \mathrm{O} & 0.60361 & 0.17418 & 0.39467 \\ \mathrm{~N} & -0.40487 & 1.92022 & -2.20555 \\ \mathrm{C} & -2.30966 & 2.33202 & -1.15428 \\ \mathrm{C} & -1.39619 & 1.37068 & -2.91749 \\ \mathrm{~N} & -2.55883 & 1.61262 & -2.30373 \\ \mathrm{~N} & -0.70353 & 0.8855 & 2.10778 \\ \mathrm{C} & -1.19462 & 1.84334 & 3.1253 \\ \mathrm{C} & -1.27524 & -0.45967 & 2.28504 \\ \mathrm{C} & -1.77585 & 0.92486 & 4.20631 \\ \mathrm{C} & -2.33362 & -0.24914 & 3.3902 \\ \mathrm{C} & 1.01678 & 1.77898 & -2.52821 \\ \mathrm{C} & 0.58863 & -3.82495 & 2.40632 \\ \mathrm{~N} & -0.29754 & -2.67909 & 2.16442 \\ \mathrm{C} & 1.97269 & -3.67136 & 1.71492 \\ \mathrm{O} & 2.85767 & -4.49449 & 1.92412 \\ \mathrm{C} & -0.08067 & -5.05893 & 1.76597 \\ \mathrm{C} & 0.8111 & -4.0796 & 3.90779 \\ \mathrm{C} & 2.86197 & 3.58671 & 0.51992 \\ \mathrm{O} & 2.42575 & 4.67949 & 0.86468 \\ \mathrm{O} & 4.13718 & 3.32056 & 0.14646 \\ \mathrm{C} & 5.1377 & 4.39925 & 0.06357 \\ \mathrm{C} & 5.36377 & 5.01922 & 1.44613 \\ \mathrm{C} & 6.38984 & 3.65828 & -0.4114 \\ \mathrm{C} & 4.70012 & 5.43906 & -0.9738 \\ \mathrm{C} & 6.35933 & -1.60991 & -3.36139 \\ \mathrm{C} & 5.11903 & -1.57155 & -2.72254 \\ \mathrm{C} & 7.09429 & -2.79547 & -3.39788 \\ \mathrm{C} & 4.59095 & -2.7171 & -2.11209 \\ \mathrm{C} & 6.58099 & -3.94258 & -2.78983 \\ \mathrm{C} & 3.25353 & -2.68679 & -1.40026 \\ & & & \\ \mathrm{n} & & & \end{array}$




$\begin{array}{lccc}\mathrm{C} & 5.3405 & -3.90159 & -2.15373 \\ \mathrm{C} & 3.37265 & -2.44099 & 0.11758 \\ \mathrm{~N} & 2.10901 & -2.65109 & 0.81408 \\ \mathrm{C} & 3.82698 & -1.01817 & 0.43796 \\ \mathrm{O} & 3.57477 & -0.0429 & -0.25174 \\ \mathrm{O} & 4.47809 & -0.96917 & 1.60248 \\ \mathrm{C} & 4.79587 & 0.33669 & 2.12136 \\ \mathrm{O} & 0.71839 & -1.10017 & 3.49655 \\ \mathrm{C} & -0.16603 & -1.44393 & 2.71522 \\ \mathrm{C} & -3.86654 & 1.20667 & -2.93414 \\ \mathrm{C} & -4.81991 & 2.36489 & -3.11024 \\ \mathrm{O} & -3.79292 & 0.25412 & -3.7005 \\ \mathrm{C} & -6.93404 & -3.0058 & -0.49566 \\ \mathrm{C} & -7.67346 & -1.73649 & -0.94091 \\ \mathrm{C} & -5.57081 & -3.1243 & -1.19051 \\ \mathrm{C} & -6.81675 & -0.48223 & -0.71451 \\ \mathrm{C} & -4.71384 & -1.86465 & -0.96692 \\ \mathrm{C} & -5.45616 & -0.59338 & -1.41897 \\ \mathrm{O} & -4.63583 & 0.54707 & -1.20487 \\ \mathrm{~N} & -3.43211 & -1.95241 & -1.66678 \\ \mathrm{C} & -2.31036 & -2.38702 & -1.06102 \\ \mathrm{O} & -2.31861 & -2.86441 & 0.08609 \\ \mathrm{C} & -5.12369 & 2.8099 & 1.12793 \\ \mathrm{C} & -6.04439 & 3.52049 & 2.11184 \\ \mathrm{O} & -3.89226 & 2.79936 & 1.318 \\ \mathrm{O} & -5.72898 & 2.2581 & 0.12821 \\ \mathrm{C} & -1.01759 & -2.23731 & -1.84119 \\ \mathrm{H} & 6.69611 & 2.90307 & 0.31813 \\ \mathrm{H} & 7.21579 & 4.36355 & -0.54241 \\ \mathrm{H} & -6.68316 & 4.2293 & 1.57662 \\ \mathrm{H} & -5.11576 & 1.35366 & -0.55652 \\ \mathrm{H} & -6.64743 & -0.33451 & 0.35913 \\ \mathrm{H} & -7.333 & 0.41626 & -1.06613 \\ \mathrm{H} & 6.20437 & 3.15815 & -1.36598 \\ \mathrm{H} & 5.62967 & 4.24357 & 2.17125 \\ \mathrm{H} & 6.19194 & 5.73362 & 1.3968 \\ \mathrm{H} & 4.47015 & 5.53697 & 1.79405 \\ \mathrm{H} & 5.49407 & 6.18027 & -1.10953 \\ \mathrm{H} & 3.7923 & 5.95118 & -0.65462 \\ \mathrm{H} & 4.51956 & 4.95775 & -1.94055 \\ \mathrm{H} & 2.52915 & 1.56441 & 0.22531 \\ \mathrm{H} & 0.73387 & 3.07444 & 1.87236 \\ \mathrm{H} & -1.01995 & 3.74849 & 0.5978 \\ \mathrm{H} & 0.34296 & 4.12946 & -0.4319 \\ \mathrm{H} & -3.06544 & 2.57508 & -0.40799 \\ \mathrm{H} & 1.46622 & 1.03967 & -1.86373 \\ \mathrm{H} & 1.10734 & 1.46566 & -3.56911 \\ \mathrm{H} & 1.51829 & 2.73738 & -2.39334 \\ \mathrm{H} & -1.2933 & 0.8284 & -3.8426 \\ \mathrm{H} & -4.93439 & 2.96151 & -2.20597 \\ \mathrm{H} & -5.47795 & 2.99648 & -3.92607 \\ \mathrm{H} & & 1.96347 & -3.40188 \\ & & & \end{array}$

$\mathrm{H} \quad-8.62362-1.63796-0.40344$

$\mathrm{H} \quad \begin{array}{llll}\mathrm{H} & -7.92665 & -1.81999 & -2.00734\end{array}$

$\mathrm{H} \quad \begin{array}{llll}-6.78593 & -2.97603 & 0.59276\end{array}$

$\mathrm{H} \quad \begin{array}{llll}-7.53889 & -3.89609 & -0.70312\end{array}$

$\mathrm{H} \quad-\quad-5.71634 \quad-3.26663 \quad-2.27041$

$\mathrm{H} \quad-\quad-5.01716 \quad-3.99555-0.82561$

$\mathrm{H} \quad-4.48522 \quad-1.77487 \quad 0.10055$

$\mathrm{H} \quad \begin{array}{llll}-5.63132 & -0.6871 & -2.50463\end{array}$

$\mathrm{H} \quad-3.35924 \quad-1.4591 \quad-2.55204$

$\mathrm{H} \quad \begin{array}{llll}-1.17809 & -1.97717 & -2.89079\end{array}$

$\mathrm{H} \quad \begin{array}{llll}-0.45511 & -3.17217 & -1.78661\end{array}$

$\mathrm{H} \quad \begin{array}{llll}-0.41624 & -1.45938 & -1.35843\end{array}$

$\mathrm{H} \quad-0.3781 \quad 2.46332 \quad 3.50226$

$\mathrm{H} \quad \begin{array}{llll}-1.97634 & 2.47719 & 2.69233\end{array}$

$\mathrm{H} \quad \begin{array}{llll}-2.5381 & 1.43187 & 4.80325\end{array}$

$\mathrm{H} \quad \begin{array}{llll}-0.97746 & 0.57575 & 4.86664\end{array}$

$\mathrm{H} \quad-2.49462 \quad-1.15436 \quad 3.98048$

$\begin{array}{llll}\mathrm{H} & -3.28238 & 0.03352 & 2.92321\end{array}$

$\mathrm{H} \quad \begin{array}{llll}-1.73028 & -0.79557 & 1.35167\end{array}$

$\mathrm{H} \quad \begin{array}{llll}-1.0137 & -2.80004 & 1.44377\end{array}$

$\mathrm{H} \quad \begin{array}{llll}-0.29836 & -4.88898 & 0.70719\end{array}$

$\mathrm{H} \quad \begin{array}{llll}-1.02411 & -5.27824 & 2.27434\end{array}$

$\mathrm{H} \quad \begin{array}{llll}0.58513 & -5.91879 & 1.85642\end{array}$

$\mathrm{H} \quad-0.14902 \quad-4.30018 \quad 4.38447$

H $\quad \begin{array}{llll}1.4786 & -4.93426 & 4.02886\end{array}$

$\mathrm{H} \quad \begin{array}{llll}\mathrm{H} & 1.25173 & -3.2071 & 4.38882\end{array}$

$\mathrm{H} \quad 1.39264-1.9362 \quad 0.74571$

$\mathrm{H} \quad 4.07815 \quad-3.14771 \quad 0.56023$

$\begin{array}{llll}\mathrm{H} & 3.90956 & 0.75911 & 2.60206\end{array}$

$\mathrm{H} \quad \begin{array}{llll}\mathrm{H} & 5.57983 & 0.17693 & 2.86075\end{array}$

$\begin{array}{llll}\mathrm{H} & 5.12973 & 1.00072 & 1.32332\end{array}$

$\mathrm{H} \quad 2.74573 \quad-3.64881 \quad-1.52206$

$\mathrm{H} \quad 2.60808 \quad-1.91448-1.83012$

$\mathrm{H} \quad \begin{array}{llll}\mathrm{H} & 4.94488 & -4.79963 & -1.68519\end{array}$

$\mathrm{H} \quad 7.14457 \quad-4.87091-2.81371$

$\mathrm{H} \quad \begin{array}{lllll}8.05865 & -2.82631 & -3.89671\end{array}$

$\mathrm{H} \quad \begin{array}{llll}6.75129 & -0.71228 & -3.83177\end{array}$

$\begin{array}{llll}\mathrm{H} & 4.5559 & -0.64402 & -2.68358\end{array}$ 


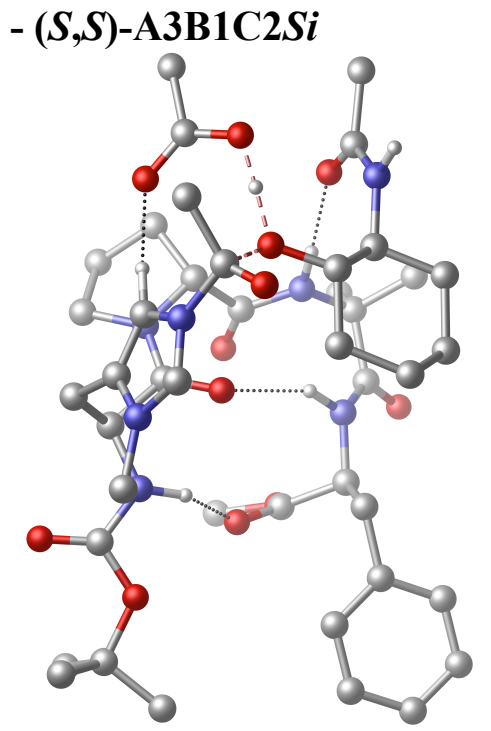

$\mathrm{N} \quad 2.25406-1.90517 \quad-0.90957$

$\begin{array}{llll}\text { C } & 1.09469 & -2.12075 & -1.75872\end{array}$

$\begin{array}{llll}\text { C } & 0.36854 & -3.46153 & -1.43228\end{array}$

$\begin{array}{lllll}\text { C } & 0.13471 & -0.93371 & -1.58491\end{array}$

$\begin{array}{llll}\text { C } & -0.32735 & -3.51326 & -0.10974\end{array}$

$\begin{array}{llll}\mathrm{O} & 0.19584 & -0.22397 & -0.57339\end{array}$

$\begin{array}{llll}\mathrm{N} & 0.26756 & -3.89495 & 1.09753\end{array}$

$\begin{array}{llll}\text { C } & -1.6274 & -3.20359 & 0.18643\end{array}$

$\begin{array}{llll}\text { C } & -0.65358 & -3.80845 & 2.06836\end{array}$

$\begin{array}{llll}\mathrm{N} & -1.80163 & -3.3946 & 1.53915\end{array}$

$\begin{array}{llll}\mathrm{N} & -0.81044 & -0.75859 & -2.53495\end{array}$

$\begin{array}{llll}\mathrm{C} & -0.86245 & -1.36148 & -3.88055\end{array}$

$\begin{array}{lllll}\text { C } & -1.82167 & 0.29668 & -2.35441\end{array}$

$\begin{array}{llll}\text { C } & -1.76329 & -0.39881 & -4.66617\end{array}$

$\begin{array}{llll}\text { C } & -2.7263 & 0.12465 & -3.59169\end{array}$

$\begin{array}{llll}\text { C } & 1.66207 & -4.2796 & 1.31521\end{array}$

$\begin{array}{llll}\mathrm{C} & -1.24657 & 3.9437 & -1.28958\end{array}$

N $\quad \begin{array}{llll}\text { C } & -1.70147 & 2.55679 & -1.46196\end{array}$

$\begin{array}{llll}\mathrm{C} & 0.20708 & 4.02187 & -0.74496\end{array}$

$\begin{array}{lllll}\text { O } & 0.8079 & 5.08955 & -0.76908\end{array}$

$\begin{array}{lllll}\text { C } & -2.1487 & 4.60248 & -0.22797\end{array}$

$\begin{array}{llll}\text { C } & -1.32769 & 4.73311 & -2.60889\end{array}$

$\begin{array}{llll}\text { C } & 3.38688 & -2.62982 & -1.15505\end{array}$

O $\quad 3.42841-3.60193-1.90247$

$\begin{array}{lllll}\text { O } & 4.41281 & -2.1614 & -0.40782\end{array}$

$\begin{array}{llll}\text { C } & 5.77596 & -2.69629 & -0.5757\end{array}$

$\begin{array}{llll}\text { C } & 6.24654 & -2.48408 & -2.01797\end{array}$

$\begin{array}{llll}\text { C } & 6.59266 & -1.83593 & 0.39046\end{array}$

$\begin{array}{llll}C & 5.82076 & -4.17065 & -0.15994\end{array}$

$\begin{array}{llll}\text { C } & 5.97372 & 1.42503 & 2.48352\end{array}$

$\begin{array}{llll}\text { C } & 4.69062 & 1.27739 & 1.95394\end{array}$

$\begin{array}{llll}\text { C } & 6.31461 & 2.57427 & 3.19775\end{array}$

$\begin{array}{llll}\text { C } & 3.72456 & 2.27601 & 2.13439\end{array}$

$\begin{array}{llll}\text { C } & 5.36208 & 3.57845 & 3.3795\end{array}$

$\begin{array}{llll}\mathrm{C} & 2.32469 & 2.13502 & 1.57184\end{array}$

$\begin{array}{llll}\text { C } & 4.0796 & 3.4271 & 2.85238\end{array}$
$\begin{array}{llll}\mathrm{C} & 2.12905 & 2.83141 & 0.21401\end{array}$

$\begin{array}{lllll}\mathrm{N} & 0.7179 & 2.89298 & -0.15921\end{array}$

$\begin{array}{lllll}\mathrm{C} & 2.85847 & 2.10913 & -0.92466\end{array}$

$\begin{array}{llll}\mathrm{O} & 3.13221 & 0.92197 & -0.94634\end{array}$

$\begin{array}{lllll}\mathrm{O} & 3.09201 & 2.9414 & -1.94497\end{array}$

$\begin{array}{llll}\text { C } & 3.5068 & 2.33115 & -3.17996\end{array}$

$\begin{array}{llll}\text { O } & -0.14616 & 1.91389 & -3.019\end{array}$

$\begin{array}{lllll}\text { C } & -1.13313 & 1.6738 & -2.32148\end{array}$

$\begin{array}{llll}\text { C } & -1.43132 & -0.40676 & 2.99215\end{array}$

$\begin{array}{lllll}\text { C } & -0.99848 & 0.65745 & 4.00983\end{array}$

$\begin{array}{llll}\text { C } & -2.933 & -0.3422 & 2.6378\end{array}$

$\begin{array}{llll}\text { C } & -1.34582 & 2.06758 & 3.52484\end{array}$

$\begin{array}{llll}\text { C } & -3.2516 & 1.08847 & 2.13463\end{array}$

$\begin{array}{llll}C & -2.8316 & 2.1566 & 3.16581\end{array}$

$\begin{array}{llll}\mathrm{O} & -3.25301 & -1.32497 & 1.6741\end{array}$

$\begin{array}{llll}\mathrm{N} & -4.65696 & 1.27464 & 1.7934\end{array}$

$\begin{array}{llll}\text { C } & -5.10896 & 1.69033 & 0.58619\end{array}$

$\begin{array}{lllll}\text { O } & -4.36888 & 1.96622 & -0.36603\end{array}$

$\begin{array}{llll}\text { C } & -6.61882 & 1.78866 & 0.46553\end{array}$

$\begin{array}{llll}\text { C } & -3.04701 & -3.16899 & 2.40205\end{array}$

$\begin{array}{llll}\text { C } & -4.27822 & -3.77385 & 1.76876\end{array}$

$\begin{array}{llll}\text { O } & -2.83732 & -3.1468 & 3.6022\end{array}$

$\begin{array}{llll}\text { C } & -5.12022 & -1.61989 & -0.92377\end{array}$

O $\quad-4.10113-2.14536-1.39104$

$\begin{array}{llll}\text { O } & -5.2645 & -1.20901 & 0.30471\end{array}$

C $\quad-6.34293-1.38785-1.79613$

$\begin{array}{llll}\mathrm{H} & 7.64355 & -2.13908 & 0.36583\end{array}$

$\begin{array}{llll}\mathrm{H} & 6.52671 & -0.78031 & 0.11425\end{array}$

$\begin{array}{llll}\mathrm{H} & 6.22384 & -1.94967 & 1.41413\end{array}$

$\begin{array}{llll}\mathrm{H} & 6.15315 & -1.42902 & -2.29267\end{array}$

$\mathrm{H} \quad \begin{array}{llll}6.3005 & -2.76687 & -2.10475\end{array}$

$\mathrm{H} \quad 5.66366 \quad-3.08622-2.71538$

$\begin{array}{llll}\mathrm{H} & 5.44094 & -4.28908 & 0.86039\end{array}$

$\mathrm{H} \quad 5.228 \quad-4.78634-0.83634$

$\mathrm{H} \quad 6.85656-4.52484-0.17477$

$\begin{array}{llll}\mathrm{H} & 2.36767 & -0.96447 & -0.53604\end{array}$

$\mathrm{H} \quad \begin{array}{llll}1.41856 & -2.20257 & -2.80279\end{array}$

$\mathrm{H} \quad-0.38409-3.64297 \quad-2.20408$

$\mathrm{H} \quad \begin{array}{llll}1.11453 & -4.25425 & -1.52909\end{array}$

$\begin{array}{llll}\mathrm{H} & 1.99801 & -4.92246 & 0.50093\end{array}$

$\begin{array}{llll}\mathrm{H} & 1.73028 & -4.82787 & 2.25549\end{array}$

$\mathrm{H} \quad 2.2947 \quad-3.39193 \quad 1.34921$

$\mathrm{H} \quad-0.50893 \quad-4.02437 \quad 3.11406$

$\mathrm{H} \quad-2.42836-2.82465-0.44083$

$\mathrm{H} \quad \begin{array}{llll}-5.16232 & -3.32643 & 2.22188\end{array}$

$\begin{array}{llll}\mathrm{H} & -4.26955 & -4.84497 & 2.00183\end{array}$

$\mathrm{H} \quad \begin{array}{llll}-4.3159 & -3.64093 & 0.68932\end{array}$

$\mathrm{H} \quad-6.37708-0.33073-2.08139$

$\mathrm{H} \quad-7.25992-1.61258-1.24521$

$\mathrm{H} \quad-6.28559-1.99619 \quad-2.69944$

$\mathrm{H} \quad-4.30902 \quad-1.23537 \quad 0.9514$

$\mathrm{H} \quad-3.51135 \quad-0.51768 \quad 3.56604$

$\mathrm{H} \quad \begin{array}{llll}-1.22096 & -1.39774 & 3.39568\end{array}$

$\mathrm{H} \quad-0.86405 \quad-0.29516 \quad 2.05857$ 


$\begin{array}{llll}\mathrm{H} & 0.07768 & 0.57216 & 4.20261 \\ \mathrm{H} & -1.50006 & 0.4704 & 4.97008 \\ \mathrm{H} & -1.09661 & 2.81718 & 4.28544 \\ \mathrm{H} & -0.7469 & 2.30868 & 2.63642 \\ \mathrm{H} & -3.08057 & 3.14497 & 2.76319 \\ \mathrm{H} & -3.43792 & 2.02853 & 4.07496 \\ \mathrm{H} & -2.69544 & 1.24248 & 1.20642 \\ \mathrm{H} & -5.34335 & 1.01938 & 2.4893 \\ \mathrm{H} & -7.02182 & 0.78204 & 0.32251 \\ \mathrm{H} & -6.86675 & 2.40472 & -0.39888 \\ \mathrm{H} & -7.07414 & 2.21954 & 1.36274 \\ \mathrm{H} & 0.13718 & -1.44406 & -4.31469 \\ \mathrm{H} & -1.30722 & -2.36404 & -3.83715 \\ \mathrm{H} & -2.27073 & -0.89777 & -5.49606 \\ \mathrm{H} & -1.16036 & 0.42259 & -5.06199 \\ \mathrm{H} & -3.222 & 1.05758 & -3.87077 \\ \mathrm{H} & -3.48973 & -0.62224 & -3.35621 \\ \mathrm{H} & -2.36994 & 0.12032 & -1.42697 \\ \mathrm{H} & -2.56848 & 2.28749 & -0.99389 \\ \mathrm{H} & -2.37091 & 4.79282 & -2.93449 \\ \mathrm{H} & -0.93709 & 5.74007 & -2.45498 \\ \mathrm{H} & -0.73861 & 4.24111 & -3.38359 \\ \mathrm{H} & -1.83221 & 5.6367 & -0.08032 \\ \mathrm{H} & -3.1924 & 4.58556 & -0.55406 \\ \mathrm{H} & -2.07855 & 4.07864 & 0.72994 \\ \mathrm{H} & 0.24672 & 2.00489 & -0.29856 \\ \mathrm{H} & 2.47846 & 3.86574 & 0.24776 \\ \mathrm{H} & 2.05702 & 1.07956 & 1.46501 \\ \mathrm{H} & 1.60599 & 2.5866 & 2.26276 \\ \mathrm{H} & 2.68121 & 1.74204 & -3.58624 \\ \mathrm{H} & 4.37964 & 1.69387 & -3.02311 \\ \mathrm{H} & 3.74667 & 3.15841 & -3.84695 \\ \mathrm{H} & 3.33965 & 4.20952 & 3.00528 \\ \mathrm{H} & 5.61485 & 4.47664 & 3.93615 \\ \mathrm{H} & 7.31284 & 2.68632 & 3.61136 \\ \mathrm{H} & 6.70888 & 0.63835 & 2.33933 \\ \mathrm{H} & 4.43505 & 0.38627 & 1.38879\end{array}$

\section{- $(R, R)-\mathrm{A} 1 \mathrm{~B} 2 \mathrm{C} 2 S i$}

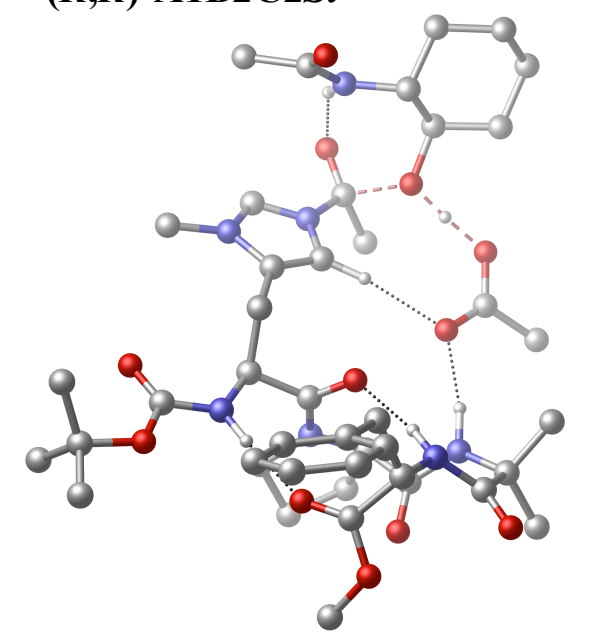

$\mathrm{N}$

C

C

C

C

$\mathrm{O}$

$\mathrm{N}$

C

C

$\mathrm{N}$

$\mathrm{N}$

C

C

C

C

C

C

$\mathrm{N}$

C

O

C

C

C

$\mathrm{O}$

$\mathrm{O}$

C

C

C

C

C

C

C

C

C

C

C

C

$\mathrm{N}$

C

$\mathrm{O}$

$\mathrm{O}$

C

$\mathrm{O}$

C

C

C

O

C

C

C

C

C

C

$\mathrm{O}$ $\begin{array}{llll}-2.36694 & 1.78961 & -0.98259\end{array}$

$\begin{array}{llll}-0.98886 & 1.426 & -1.25218\end{array}$

$\begin{array}{lll}-0.01121 & 1.9832 & -0.18382\end{array}$

$\begin{array}{lll}-0.82829 & -0.1084 & -1.27776\end{array}$

$\begin{array}{llll}1.40739 & 1.92098 & -0.65064\end{array}$

$\begin{array}{llll}-0.49644 & -0.69662 & -0.24066\end{array}$

$2.06123 .03036-1.19301$

$\begin{array}{llll}2.28946 & 0.87366 & -0.73633\end{array}$

$\begin{array}{llll}3.29423 & 2.66063 & -1.56205\end{array}$

$\begin{array}{llll}3.45186 & 1.36414 & -1.3\end{array}$

$\begin{array}{llll}-0.97799 & -0.74592 & -2.45469\end{array}$

$\begin{array}{llll}-1.56678 & -0.19168 & -3.6851\end{array}$

$\begin{array}{llll}-0.5499 & -2.15782 & -2.60953\end{array}$

$\begin{array}{llll}-1.94989 & -1.45117 & -4.46926\end{array}$

$\begin{array}{llll}-0.83609 & -2.44387 & -4.10014\end{array}$

$\begin{array}{llll}1.51364 & 4.38489 & -1.30666\end{array}$

$\begin{array}{llll}-1.1321 & -4.61511 & 0.2822\end{array}$

$\begin{array}{lll}-0.58516 & -3.70132 & -0.73308\end{array}$

$\begin{array}{llll}-2.3021 & -3.96655 & 1.07896\end{array}$

$\begin{array}{lll}-3.16693 & -4.66012 & 1.59771\end{array}$

$\begin{array}{lll}-0.01177 & -4.92088 & 1.29459\end{array}$

$\begin{array}{lll}-1.6364 & -5.9123 & -0.36966\end{array}$

$\begin{array}{llll}-2.76695 & 3.07303 & -1.20424\end{array}$

$\begin{array}{llll}-2.02588 & 3.9493 & -1.64741\end{array}$

$\begin{array}{llll}-4.06521 & 3.2221 & -0.86915\end{array}$

$-4.75575 \quad 4.51516-1.02525$

$\begin{array}{llll}-4.76715 & 4.92778 & -2.50055\end{array}$

$-6.171164 .19264-0.54175$

$-4.09975 \quad 5.5703 \quad-0.12948$

$\begin{array}{llll}-5.73044 & 1.82074 & 3.40553\end{array}$

$\begin{array}{lll}-4.66091 & 1.19493 & 2.76266\end{array}$

$\begin{array}{lll}-6.23976 & 1.30012 & 4.59527\end{array}$

$\begin{array}{lll}-4.08006 & 0.03888 & 3.30109\end{array}$

$\begin{array}{lll}-5.67061 & 0.14785 & 5.14059\end{array}$

$\begin{array}{lll}-2.93028 & -0.66486 & 2.60881\end{array}$

$\begin{array}{lll}-4.60074 & -0.47394 & 4.49802\end{array}$

$\begin{array}{lll}-3.37811 & -1.86405 & 1.7559\end{array}$

$\begin{array}{lll}-2.23452 & -2.60814 & 1.23856\end{array}$

$\begin{array}{llll}-4.20303 & -1.43515 & 0.53629\end{array}$

$\begin{array}{llll}-4.00095 & -0.42974 & -0.12897\end{array}$

$\begin{array}{llll}-5.12554 & -2.34317 & 0.22678\end{array}$

$\begin{array}{llll}-5.77228 & -2.18511 & -1.05143\end{array}$

$-2.55182-3.28184-1.83945$

$\begin{array}{llll}-1.34189 & -3.10147 & -1.68291\end{array}$

$\begin{array}{llll}4.75973 & 0.65805 & -1.66545\end{array}$

$\begin{array}{llll}4.5167 & -0.4807 & -2.63003\end{array}$

$\begin{array}{llll}5.73247 & 1.40314 & -1.72914\end{array}$

$\begin{array}{llll}7.42337 & -0.2386 & 3.37111\end{array}$

$\begin{array}{llll}7.61994 & -1.34787 & 2.33297\end{array}$

$\begin{array}{lll}7.08405 & 1.0845 & 2.67885\end{array}$

$\begin{array}{llll}6.38161 & -1.48622 & 1.43787\end{array}$

$\begin{array}{llll}5.84568 & 0.97358 & 1.7625\end{array}$

$\begin{array}{llll}6.00642 & -0.16707 & 0.73887\end{array}$

$\begin{array}{llll}4.79949 & -0.33883 & -0.01871\end{array}$ 


\begin{tabular}{|c|c|c|c|}
\hline $\mathrm{N}$ & 5.61947 & 2.24453 & 1.07461 \\
\hline C & 5.13346 & 3.33878 & 1.71627 \\
\hline $\mathrm{O}$ & 4.76534 & 3.33157 & 2.88856 \\
\hline C & 3.24365 & -3.17684 & -0.69871 \\
\hline $\mathrm{C}$ & 3.20334 & -4.68828 & -0.89663 \\
\hline $\mathrm{O}$ & 2.18803 & -2.50202 & -0.76403 \\
\hline $\mathrm{O}$ & 4.41057 & -2.70187 & -0.4674 \\
\hline $\mathrm{C}$ & 5.08236 & 4.61924 & 0.88627 \\
\hline $\mathrm{H}$ & -6.6173 & 3.40372 & -1.15337 \\
\hline $\mathrm{H}$ & -6.8022 & 5.08371 & -0.6076 \\
\hline $\mathrm{H}$ & -6.15641 & 3.85337 & 0.49779 \\
\hline $\mathrm{H}$ & -5.20182 & 4.13195 & -3.1134 \\
\hline $\mathrm{H}$ & -5.38187 & 5.82498 & -2.62468 \\
\hline $\mathrm{H}$ & -3.75892 & 5.13976 & -2.85689 \\
\hline $\mathrm{H}$ & -4.68651 & 6.49386 & -0.16076 \\
\hline $\mathrm{H}$ & -3.08406 & 5.7879 & -0.46057 \\
\hline $\mathrm{H}$ & -4.0696 & 5.22313 & 0.90795 \\
\hline $\mathrm{H}$ & -3.01296 & 1.10623 & -0.58734 \\
\hline $\mathrm{H}$ & -0.71542 & 1.84558 & -2.22582 \\
\hline $\mathrm{H}$ & -0.29527 & 3.01287 & 0.03533 \\
\hline $\mathrm{H}$ & -0.12366 & 1.38873 & 0.72364 \\
\hline $\mathrm{H}$ & 2.18209 & -0.16712 & -0.45438 \\
\hline $\mathrm{H}$ & 1.52308 & 4.87608 & -0.33033 \\
\hline $\mathrm{H}$ & 2.13278 & 4.95387 & -2.00073 \\
\hline $\mathrm{H}$ & 0.48765 & 4.34131 & -1.67806 \\
\hline $\mathrm{H}$ & 4.04951 & 3.292 & -1.99874 \\
\hline $\mathrm{H}$ & 3.63617 & -1.06953 & -2.37526 \\
\hline $\mathrm{H}$ & 4.38775 & -0.03996 & -3.6253 \\
\hline $\mathrm{H}$ & 5.39417 & -1.12687 & -2.64529 \\
\hline $\mathrm{H}$ & 3.32633 & -5.18196 & 0.07338 \\
\hline $\mathrm{H}$ & 2.25806 & -5.00637 & -1.33969 \\
\hline $\mathrm{H}$ & 4.03851 & -5.00533 & -1.52608 \\
\hline $\mathrm{H}$ & 4.56855 & -1.42652 & -0.2178 \\
\hline $\mathrm{H}$ & 5.52539 & -1.80786 & 2.04461 \\
\hline $\mathrm{H}$ & 6.52823 & -2.26183 & 0.68211 \\
\hline $\mathrm{H}$ & 7.82611 & -2.30791 & 2.82025 \\
\hline $\mathrm{H}$ & 8.49929 & -1.11621 & 1.71538 \\
\hline $\mathrm{H}$ & 6.60927 & -0.51751 & 4.05425 \\
\hline $\mathrm{H}$ & 8.32184 & -0.11974 & 3.98791 \\
\hline $\mathrm{H}$ & 7.93829 & 1.40689 & 2.06688 \\
\hline $\mathrm{H}$ & 6.89237 & 1.87626 & 3.40819 \\
\hline $\mathrm{H}$ & 4.96388 & 0.76353 & 2.37936 \\
\hline $\mathrm{H}$ & 6.81209 & 0.11382 & 0.0408 \\
\hline $\mathrm{H}$ & 5.8814 & 2.29217 & 0.09525 \\
\hline $\mathrm{H}$ & 4.05937 & 5.00699 & 0.88942 \\
\hline $\mathrm{H}$ & 5.42812 & 4.49563 & -0.14404 \\
\hline $\mathrm{H}$ & 5.70839 & 5.3712 & 1.37472 \\
\hline $\mathrm{H}$ & -2.42119 & 0.44599 & -3.44784 \\
\hline $\mathrm{H}$ & -0.82604 & 0.40629 & -4.23542 \\
\hline $\mathrm{H}$ & -2.02533 & -1.26338 & -5.54359 \\
\hline $\mathrm{H}$ & -2.9065 & -1.83293 & -4.1048 \\
\hline $\mathrm{H}$ & -1.12994 & -3.48246 & -4.26365 \\
\hline $\mathrm{H}$ & 0.06609 & -2.24885 & -4.6904 \\
\hline $\mathrm{H}$ & 0.51386 & -2.22389 & -2.3667 \\
\hline
\end{tabular}

$\begin{array}{llll}\text { H } & 0.37848 & -3.36754 & -0.63015 \\ \mathrm{H} & 0.3924 & -4.00199 & 1.72994 \\ \mathrm{H} & 0.80389 & -5.46508 & 0.8095 \\ \mathrm{H} & -0.41135 & -5.54148 & 2.09998 \\ \mathrm{H} & -0.8003 & -6.43049 & -0.84931 \\ \mathrm{H} & -2.07932 & -6.55863 & 0.38914 \\ \mathrm{H} & -2.39723 & -5.68711 & -1.11831 \\ \mathrm{H} & -1.54935 & -2.08021 & 0.70221 \\ \mathrm{H} & -3.97168 & -2.56719 & 2.34293 \\ \mathrm{H} & -5.03363 & -2.32431 & -1.84329 \\ \mathrm{H} & -6.52919 & -2.96736 & -1.08996 \\ \mathrm{H} & -6.23066 & -1.19714 & -1.13102 \\ \mathrm{H} & -2.22245 & -1.04843 & 3.35069 \\ \mathrm{H} & -2.38144 & 0.03293 & 1.96925 \\ \mathrm{H} & -4.15801 & -1.3671 & 4.93269 \\ \mathrm{H} & -6.05627 & -0.26437 & 6.06869 \\ \mathrm{H} & -7.07079 & 1.78885 & 5.09558 \\ \mathrm{H} & -6.16568 & 2.71915 & 2.97607 \\ \mathrm{H} & -4.27826 & 1.60567 & 1.83294\end{array}$

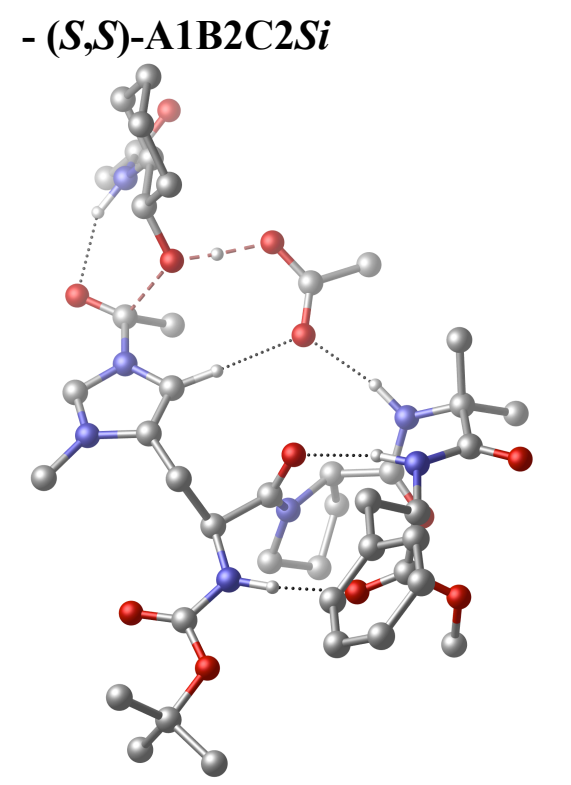

$\begin{array}{llll}\mathrm{N} & -2.79454 & 1.87979 & -0.63906 \\ \mathrm{C} & -1.35646 & 1.7527 & -0.77656 \\ \mathrm{C} & -0.60064 & 2.21034 & 0.5007 \\ \mathrm{C} & -0.96087 & 0.28448 & -1.04553 \\ \mathrm{C} & 0.84904 & 2.43952 & 0.21777 \\ \mathrm{O} & -0.6738 & -0.44256 & -0.0858 \\ \mathrm{~N} & 1.38179 & 3.72262 & 0.05429 \\ \mathrm{C} & 1.87202 & 1.56133 & -0.0347 \\ \mathrm{C} & 2.67721 & 3.61284 & -0.26188 \\ \mathrm{~N} & 2.99104 & 2.31822 & -0.33137 \\ \mathrm{~N} & -0.8573 & -0.12713 & -2.32296 \\ \mathrm{C} & -1.36995 & 0.57126 & -3.51377 \\ \mathrm{C} & -0.19704 & -1.4154 & -2.65391 \\ \mathrm{C} & -1.44946 & -0.55153 & -4.55357 \\ \mathrm{C} & -0.24932 & -1.44336 & -4.19716\end{array}$




\begin{tabular}{|c|c|c|c|}
\hline $\mathrm{C}$ & 0.65226 & 4.98439 & 0.20808 \\
\hline $\mathrm{C}$ & -0.74447 & -4.42757 & -0.33885 \\
\hline $\mathrm{N}$ & -0.22252 & -3.27506 & -1.09125 \\
\hline $\mathrm{C}$ & -2.10204 & -4.10323 & 0.35011 \\
\hline $\mathrm{O}$ & -2.91074 & -4.98934 & 0.59174 \\
\hline $\mathrm{C}$ & 0.26108 & -4.75872 & 0.78007 \\
\hline $\mathrm{C}$ & -0.93643 & -5.63795 & -1.26631 \\
\hline $\mathrm{C}$ & -3.35266 & 3.12212 & -0.66922 \\
\hline $\mathrm{O}$ & -2.70136 & 4.15656 & -0.81021 \\
\hline $\mathrm{O}$ & -4.69072 & 3.03782 & -0.52003 \\
\hline $\mathrm{C}$ & -5.54216 & 4.24153 & -0.52652 \\
\hline $\mathrm{C}$ & -5.41916 & 4.96364 & -1.87211 \\
\hline $\mathrm{C}$ & -6.94479 & 3.65539 & -0.3533 \\
\hline $\mathrm{C}$ & -5.17691 & 5.14396 & 0.65587 \\
\hline $\mathrm{C}$ & -6.72184 & 0.60236 & 3.03157 \\
\hline $\mathrm{C}$ & -5.48463 & 0.2697 & 2.47668 \\
\hline $\mathrm{C}$ & -7.2995 & -0.21291 & 4.00502 \\
\hline $\mathrm{C}$ & -4.80247 & -0.8826 & 2.88938 \\
\hline $\mathrm{C}$ & -6.63046 & -1.36506 & 4.42237 \\
\hline $\mathrm{C}$ & -3.46656 & -1.2698 & 2.28796 \\
\hline $\mathrm{C}$ & -5.39381 & -1.6938 & 3.86875 \\
\hline $\mathrm{C}$ & -3.58469 & -2.33068 & 1.18029 \\
\hline $\mathrm{N}$ & -2.2735 & -2.80393 & 0.75053 \\
\hline $\mathrm{C}$ & -4.28268 & -1.78812 & -0.07324 \\
\hline $\mathrm{O}$ & -4.14702 & -0.65706 & -0.51773 \\
\hline $\mathrm{O}$ & -4.99664 & -2.73134 & -0.6817 \\
\hline $\mathrm{C}$ & -5.46788 & -2.41942 & -2.00774 \\
\hline $\mathrm{O}$ & -2.07736 & -2.92049 & -2.3945 \\
\hline $\mathrm{C}$ & -0.93903 & -2.61394 & -2.03091 \\
\hline $\mathrm{C}$ & 5.08036 & 0.27285 & 3.1055 \\
\hline $\mathrm{C}$ & 6.12735 & 0.48134 & 4.20831 \\
\hline $\mathrm{C}$ & 5.53915 & 0.81522 & 1.74161 \\
\hline $\mathrm{C}$ & 7.47817 & -0.12194 & 3.80155 \\
\hline C & 6.90236 & 0.21449 & 1.33875 \\
\hline $\mathrm{C}$ & 7.94447 & 0.44089 & 2.45417 \\
\hline $\mathrm{O}$ & 4.51107 & 0.58124 & 0.78393 \\
\hline $\mathrm{N}$ & 7.35155 & 0.79091 & 0.07277 \\
\hline C & 8.34758 & 0.23355 & -0.67064 \\
\hline $\mathrm{O}$ & 8.94184 & -0.78971 & -0.34281 \\
\hline $\mathrm{C}$ & 8.68969 & 0.98023 & -1.95587 \\
\hline $\mathrm{C}$ & 4.37064 & 1.88828 & -0.76577 \\
\hline $\mathrm{C}$ & 4.34806 & 0.83093 & -1.83877 \\
\hline $\mathrm{O}$ & 5.22791 & 2.7485 & -0.63725 \\
\hline $\mathrm{C}$ & 3.45566 & -2.32232 & -0.38814 \\
\hline $\mathrm{O}$ & 2.35546 & -1.74198 & -0.60213 \\
\hline $\mathrm{O}$ & 4.481 & -1.7883 & 0.14591 \\
\hline $\mathrm{C}$ & 3.62744 & -3.78708 & -0.7826 \\
\hline $\mathrm{H}$ & -7.68809 & 4.45791 & -0.34586 \\
\hline $\mathrm{H}$ & -7.18048 & 2.97013 & -1.17207 \\
\hline $\mathrm{H}$ & -7.0206 & 3.10356 & 0.58781 \\
\hline $\mathrm{H}$ & -5.64428 & 4.27616 & -2.69333 \\
\hline $\mathrm{H}$ & -6.1396 & 5.78682 & -1.91297 \\
\hline $\mathrm{H}$ & -4.41593 & 5.3666 & -2.01149 \\
\hline $\mathrm{H}$ & -5.23608 & 4.58335 & 1.59393 \\
\hline
\end{tabular}

\begin{tabular}{|c|c|c|c|}
\hline $\mathrm{H}$ & -4.16928 & 5.54508 & 0.5445 \\
\hline $\mathrm{H}$ & -5.88364 & 5.97788 & 0.71423 \\
\hline $\mathrm{H}$ & -3.37476 & 1.05188 & -0.49977 \\
\hline $\mathrm{H}$ & -1.0383 & 2.38511 & -1.61196 \\
\hline $\mathrm{H}$ & -1.06844 & 3.12489 & 0.86517 \\
\hline $\mathrm{H}$ & -0.71164 & 1.43121 & 1.25632 \\
\hline $\mathrm{H}$ & -0.30035 & 4.92723 & -0.32298 \\
\hline $\mathrm{H}$ & 1.25878 & 5.78988 & -0.20651 \\
\hline $\mathrm{H}$ & 0.46483 & 5.1832 & 1.26615 \\
\hline $\mathrm{H}$ & 3.37178 & 4.41756 & -0.438 \\
\hline $\mathrm{H}$ & 1.90308 & 0.47612 & -0.01654 \\
\hline $\mathrm{H}$ & 5.32264 & 0.3449 & -1.86408 \\
\hline $\mathrm{H}$ & 4.17852 & 1.34026 & -2.79549 \\
\hline $\mathrm{H}$ & 3.57384 & 0.08021 & -1.68005 \\
\hline $\mathrm{H}$ & 3.6481 & -4.40542 & 0.12112 \\
\hline $\mathrm{H}$ & 4.58952 & -3.92324 & -1.28296 \\
\hline $\mathrm{H}$ & 2.81755 & -4.12632 & -1.43112 \\
\hline $\mathrm{H}$ & 4.45505 & -0.46317 & 0.50162 \\
\hline $\mathrm{H}$ & 5.65625 & 1.90875 & 1.80693 \\
\hline $\mathrm{H}$ & 4.12958 & 0.75175 & 3.36461 \\
\hline $\mathrm{H}$ & 4.87231 & -0.79839 & 2.98722 \\
\hline $\mathrm{H}$ & 5.77118 & 0.04162 & 5.14751 \\
\hline $\mathrm{H}$ & 6.2531 & 1.55756 & 4.39584 \\
\hline $\mathrm{H}$ & 8.23346 & 0.07242 & 4.5721 \\
\hline $\mathrm{H}$ & 7.38165 & -1.21376 & 3.72709 \\
\hline $\mathrm{H}$ & 8.88302 & -0.02107 & 2.13722 \\
\hline $\mathrm{H}$ & 8.12773 & 1.52108 & 2.54763 \\
\hline $\mathrm{H}$ & 6.77879 & -0.86438 & 1.18467 \\
\hline $\mathrm{H}$ & 6.90663 & 1.64353 & -0.24609 \\
\hline $\mathrm{H}$ & 8.00692 & 1.80519 & -2.17792 \\
\hline $\mathrm{H}$ & 8.68092 & 0.26845 & -2.78533 \\
\hline $\mathrm{H}$ & 9.70764 & 1.3736 & -1.87715 \\
\hline $\mathrm{H}$ & -2.33717 & 1.0323 & -3.30144 \\
\hline $\mathrm{H}$ & -0.67068 & 1.35686 & -3.83454 \\
\hline $\mathrm{H}$ & -1.41712 & -0.16982 & -5.5775 \\
\hline $\mathrm{H}$ & -2.37322 & -1.11747 & -4.4127 \\
\hline $\mathrm{H}$ & -0.35528 & -2.46008 & -4.57999 \\
\hline $\mathrm{H}$ & 0.67768 & -1.02269 & -4.60198 \\
\hline $\mathrm{H}$ & 0.82608 & -1.38881 & -2.26975 \\
\hline $\mathrm{H}$ & 0.66605 & -2.85156 & -0.80347 \\
\hline $\mathrm{H}$ & 0.02943 & -5.93331 & -1.6873 \\
\hline $\mathrm{H}$ & -1.36005 & -6.47165 & -0.70472 \\
\hline $\mathrm{H}$ & -1.62049 & -5.38659 & -2.07824 \\
\hline $\mathrm{H}$ & -0.1384 & -5.56581 & 1.39862 \\
\hline $\mathrm{H}$ & 1.21308 & -5.08718 & 0.35431 \\
\hline $\mathrm{H}$ & 0.44598 & -3.88958 & 1.41847 \\
\hline $\mathrm{H}$ & -1.61405 & -2.09803 & 0.43011 \\
\hline $\mathrm{H}$ & -4.13535 & -3.20624 & 1.52871 \\
\hline $\mathrm{H}$ & -2.96071 & -0.39039 & 1.87776 \\
\hline $\mathrm{H}$ & -2.81334 & -1.68869 & 3.06034 \\
\hline $\mathrm{H}$ & -4.60869 & -2.30898 & -2.67206 \\
\hline $\mathrm{H}$ & -6.06097 & -1.5025 & -1.99908 \\
\hline $\mathrm{H}$ & -6.07713 & -3.27196 & -2.30471 \\
\hline $\mathrm{H}$ & -4.87555 & -2.58956 & 4.20285 \\
\hline
\end{tabular}




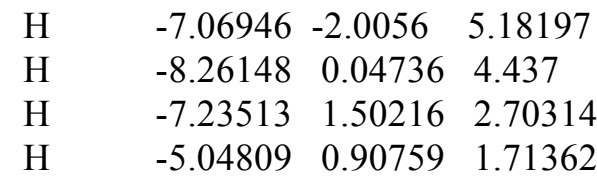

- $(R, R)-\mathrm{A} 2 \mathrm{~B} 2 \mathrm{C} 2 \mathrm{Si}$

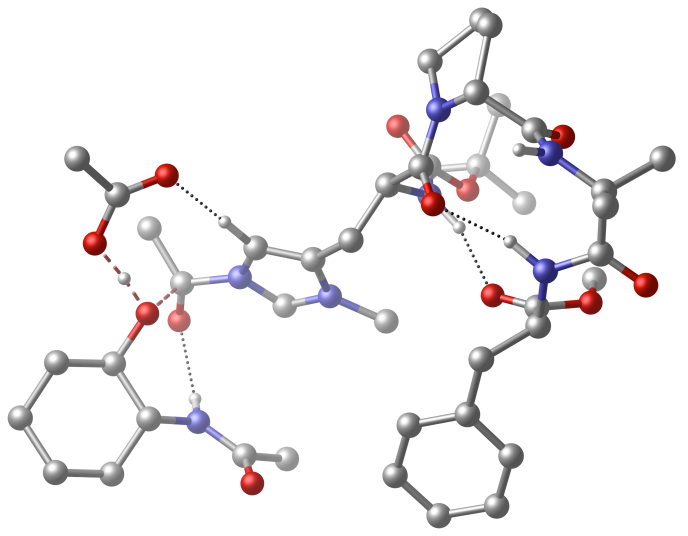

$\begin{array}{llll}\mathrm{N} & 1.57511 & -1.45858 & 1.03785\end{array}$

$\begin{array}{llll}\mathrm{C} & 1.06602 & -1.64151 & -0.31286\end{array}$

$\begin{array}{llll}\text { C } & -0.08159 & -0.6537 & -0.62618\end{array}$

$\begin{array}{llll}\text { C } & 2.21448 & -1.40232 & -1.31946\end{array}$

$\begin{array}{llll}\text { C } & -1.32414 & -0.83885 & 0.18685\end{array}$

$\begin{array}{lllll}\text { O } & 2.35264 & -0.29666 & -1.86463\end{array}$

$\begin{array}{llll}\mathrm{N} & -1.57317 & -0.24713 & 1.43343\end{array}$

$\begin{array}{llll}\text { C } & -2.45928 & -1.52617 & -0.14598\end{array}$

$\begin{array}{llll}\text { C } & -2.81192 & -0.57585 & 1.8148\end{array}$

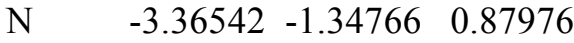

$\begin{array}{lllll}\mathrm{N} & 3.08025 & -2.40937 & -1.56185\end{array}$

$\begin{array}{llll}\text { C } & 3.09998 & -3.76632 & -0.98159\end{array}$

$\begin{array}{llll}\text { C } & 4.25013 & -2.14694 & -2.4134\end{array}$

$\begin{array}{llll}\text { C } & 4.57956 & -4.1491 & -1.07915\end{array}$

$\begin{array}{llll}\text { C } & 5.0212 & -3.48885 & -2.39474\end{array}$

$\begin{array}{lllll}\text { C } & -0.67823 & 0.60249 & 2.22403\end{array}$

$\begin{array}{llll}\mathrm{C} & 6.28499 & 1.13953 & -2.43691\end{array}$

N $\quad \begin{array}{llll}\text { N } & 5.48057 & -0.05908 & -2.72685\end{array}$

C $\quad 5.55552 \quad 2.14818-1.50158$

O $\quad 6.18531 \quad 3.07146 \quad-0.99906$

$\begin{array}{llll}\text { C } & 6.50991 & 1.86907 & -3.77547\end{array}$

$\begin{array}{lllll}\text { C } & & 7.63525 & 0.75911 & -1.8078\end{array}$

$\begin{array}{llll}C & 1.53087 & -2.43151 & 1.99187\end{array}$

$\begin{array}{llll}\text { O } & 1.16719 & -3.5842 & 1.80048\end{array}$

$\begin{array}{llll}\text { O } & 1.94289 & -1.91087 & 3.17744\end{array}$

$\begin{array}{llll}\text { C } & 2.07207 & -2.76439 & 4.37522\end{array}$

$\begin{array}{llll}\text { C } & 3.08589 & -3.8831 & 4.1164\end{array}$

$\begin{array}{llll}\text { C } & 2.6018 & -1.78586 & 5.42562\end{array}$

$\begin{array}{llll}\text { C } & 0.69773 & -3.30623 & 4.78011\end{array}$

$\begin{array}{llll}\text { C } & -1.09637 & 4.60539 & 0.45815\end{array}$

$\begin{array}{llll}\text { C } & -0.2001 & 3.83044 & -0.28208\end{array}$

$\begin{array}{llll}\text { C } & -0.61397 & 5.66755 & 1.22661\end{array}$

$\begin{array}{llll}\text { C } & 1.1757 & 4.10221 & -0.28288\end{array}$

$\begin{array}{llll}\text { C } & 0.75274 & 5.95024 & 1.23978\end{array}$
C

$\mathrm{N}$

C

$\mathrm{O}$

$\mathrm{O}$

C

$\mathrm{O}$

C

C

C

$\mathrm{O}$

C

C

C

C

C

C

O

$\mathrm{N}$

C

O

C

C

$\mathrm{O}$

O

C

$\mathrm{H}$

$\mathrm{H}$

H

H

H

H

$\mathrm{H}$

$\mathrm{H}$

$\mathrm{H}$

$\mathrm{H}$

$\mathrm{H}$

$\mathrm{H}$

$\mathrm{H}$

$\mathrm{H}$

$\mathrm{H}$

H

H

$\mathrm{H}$

H

H

$\mathrm{H}$

$\mathrm{H}$

$\mathrm{H}$

$\mathrm{H}$

$\mathrm{H}$

H $\begin{array}{llll}2.10982 & 3.27172 & -1.14331\end{array}$

$\begin{array}{llll}1.63951 & 5.17774 & 0.48755\end{array}$

$\begin{array}{llll}3.43155 & 2.85783 & -0.47797\end{array}$

$\begin{array}{llll}4.20559 & 2.00113 & -1.3714\end{array}$

$\begin{array}{llll}3.21922 & 2.0725 & 0.81913\end{array}$

$\begin{array}{lll}2.26048 & 1.34943 & 1.04574\end{array}$

$\begin{array}{lll}4.24993 & 2.21355 & 1.65339\end{array}$

$\begin{array}{llll}4.23609 & 1.39153 & 2.83598\end{array}$

$\begin{array}{llll}5.39686 & -0.98196 & -0.6306\end{array}$

$\begin{array}{llll}5.0918 & -0.99836 & -1.81817\end{array}$

$\begin{array}{llll}-4.80078 & -1.79217 & 0.98029\end{array}$

$\begin{array}{llll}-5.02189 & -3.20526 & 0.50384\end{array}$

$\begin{array}{lll}-5.44535 & -1.25492 & 1.87355\end{array}$

$\begin{array}{llll}-8.17501 & 2.12288 & -1.70861\end{array}$

$\begin{array}{llll}-8.55365 & 0.65022 & -1.90234\end{array}$

$\begin{array}{llll}-7.33677 & 2.29714 & -0.43798\end{array}$

$\begin{array}{llll}-7.30313 & -0.23647 & -1.93568\end{array}$

$\begin{array}{llll}-6.08104 & 1.40051 & -0.42935\end{array}$

$\begin{array}{llll}-6.43311 & -0.08292 & -0.67363\end{array}$

$\begin{array}{llll}-5.253 & -0.86741 & -0.75753\end{array}$

$\begin{array}{lll}-5.38554 & 1.54096 & 0.85245\end{array}$

$\begin{array}{lll}-4.64599 & 2.62963 & 1.17051\end{array}$

$\begin{array}{lll}-4.39941 & 3.54083 & 0.37703\end{array}$

$-4.30628-2.99913-3.11508$

$-4.51253-3.68868-4.45901$

$\begin{array}{llll}-3.24815 & -3.18399 & -2.48344\end{array}$

$\begin{array}{llll}-5.29384 & -2.25928 & -2.74289\end{array}$

$\begin{array}{lll}-4.13824 & 2.6938 & 2.6077\end{array}$

$\begin{array}{llll}3.56839 & -1.37456 & 5.12058\end{array}$

$\begin{array}{llll}2.73276 & -2.299 & 6.3826\end{array}$

$\begin{array}{llll}1.9023 & -0.95765 & 5.57205\end{array}$

$\begin{array}{llll}4.03672 & -3.46258 & 3.77417\end{array}$

$\begin{array}{llll}3.27208 & -4.42898 & 5.04662\end{array}$

$\begin{array}{llll}2.7161 & -4.58328 & 3.36732\end{array}$

$\begin{array}{llll}0.78346 & -3.85809 & 5.72149\end{array}$

$\begin{array}{llll}0.30005 & -3.9735 & 4.01549\end{array}$

$\begin{array}{lll}-0.00582 & -2.48199 & 4.93598\end{array}$

$\begin{array}{llll}1.9484 & -0.54475 & 1.28145\end{array}$

$\begin{array}{llll}0.70057 & -2.66892 & -0.37527\end{array}$

$\begin{array}{llll}0.30848 & 0.36167 & -0.5238\end{array}$

$\begin{array}{llll}-0.35954 & -0.77347 & -1.67686\end{array}$

$\begin{array}{lll}-2.71002 & -2.098 & -1.04382\end{array}$

$\begin{array}{lll}-0.18439 & 1.32823 & 1.57856\end{array}$

$\begin{array}{lll}-1.27806 & 1.13343 & 2.96349\end{array}$

$\begin{array}{lll}0.07684 & -0.00341 & 2.72543\end{array}$

$\begin{array}{llll}-3.30017 & -0.27381 & 2.72461\end{array}$

$\begin{array}{llll}-4.45364 & -3.44944 & -0.39369\end{array}$

$\begin{array}{llll}-4.72545 & -3.87992 & 1.3163\end{array}$

$\begin{array}{lll}-6.08709 & -3.34148 & 0.31576\end{array}$

$\begin{array}{llll}-3.6834 & -4.36273 & -4.67791\end{array}$

$\begin{array}{llll}-5.45556 & -4.24324 & -4.4532\end{array}$

$\begin{array}{llll}-4.59053 & -2.93402 & -5.24849\end{array}$

$\begin{array}{llll}-5.22291 & -1.56056 & -1.69478\end{array}$

$\begin{array}{llll}-6.69317 & 0.01776 & -2.811\end{array}$ 


$\begin{array}{llll}\mathrm{H} & -7.5707 & -1.2902 & -2.05398 \\ \mathrm{H} & -9.12561 & 0.51483 & -2.82788 \\ \mathrm{H} & -9.21151 & 0.33394 & -1.0801 \\ \mathrm{H} & -7.60154 & 2.46946 & -2.5796 \\ \mathrm{H} & -9.07156 & 2.75194 & -1.65476 \\ \mathrm{H} & -7.94797 & 2.0408 & 0.43902 \\ \mathrm{H} & -7.01669 & 3.33605 & -0.31359 \\ \mathrm{H} & -5.39495 & 1.72883 & -1.21823 \\ \mathrm{H} & -7.01905 & -0.42666 & 0.19833 \\ \mathrm{H} & -5.5215 & 0.79626 & 1.52859 \\ \mathrm{H} & -4.38294 & 1.81194 & 3.20689 \\ \mathrm{H} & -4.58477 & 3.56884 & 3.08899 \\ \mathrm{H} & -3.05632 & 2.85378 & 2.5994 \\ \mathrm{H} & 2.71248 & -3.7752 & 0.03605 \\ \mathrm{H} & 2.4782 & -4.43943 & -1.58773 \\ \mathrm{H} & 4.73046 & -5.23153 & -1.07141 \\ \mathrm{H} & 5.12842 & -3.70773 & -0.24315 \\ \mathrm{H} & 6.10174 & -3.3406 & -2.46014 \\ \mathrm{H} & 4.71396 & -4.09652 & -3.25265 \\ \mathrm{H} & 3.91649 & -1.88283 & -3.42315 \\ \mathrm{H} & 5.05882 & -0.10884 & -3.64311 \\ \mathrm{H} & 5.55755 & 2.13757 & -4.24679 \\ \mathrm{H} & 7.08049 & 1.23721 & -4.46372 \\ \mathrm{H} & 7.06956 & 2.78838 & -3.59562 \\ \mathrm{H} & 8.2093 & 0.14826 & -2.51125 \\ \mathrm{H} & 8.1912 & 1.66869 & -1.57768 \\ \mathrm{H} & 7.48306 & 0.19471 & -0.88774 \\ \mathrm{H} & 3.73872 & 1.16694 & -1.71749 \\ \mathrm{H} & 4.06761 & 3.71635 & -0.2543 \\ \mathrm{H} & 4.27969 & 0.33485 & 2.56215 \\ \mathrm{H} & 5.1253 & 1.67698 & 3.39589 \\ \mathrm{H} & 3.33336 & 1.57845 & 3.42163 \\ \mathrm{H} & 2.37917 & 3.82755 & -2.05043 \\ \mathrm{H} & 1.59359 & 2.36589 & -1.47466 \\ \mathrm{H} & 2.69784 & 5.42543 & 0.49691 \\ \mathrm{H} & 1.13206 & 6.77992 & 1.83005 \\ \mathrm{H} & -1.30272 & 6.27685 & 1.8048 \\ \mathrm{H} & -2.16148 & 4.38474 & 0.42652 \\ \mathrm{H} & -0.57898 & 3.01046 & -0.88812 \\ & & & \\ & & & \end{array}$

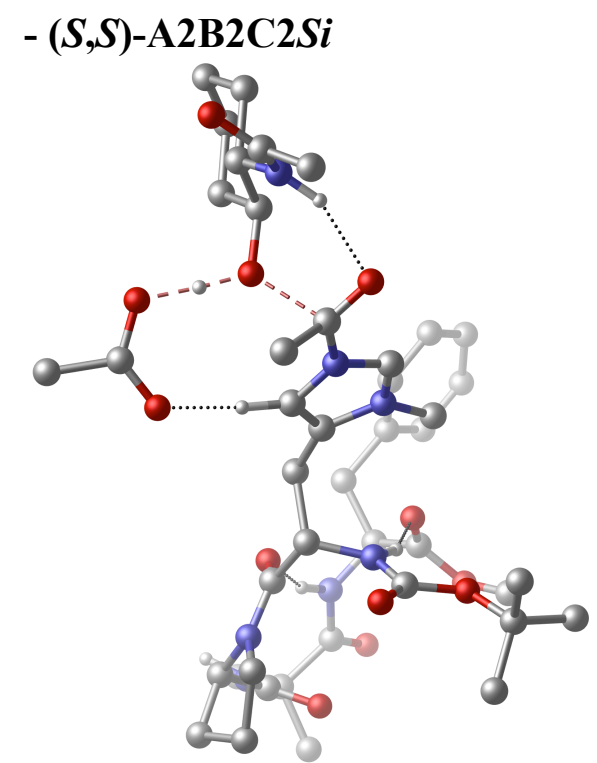

$\begin{array}{llcc}\mathrm{N} & 1.48986 & -1.30693 & 0.99885 \\ \mathrm{C} & 0.96547 & -1.35685 & -0.35752 \\ \mathrm{C} & 0.00598 & -0.17486 & -0.62879 \\ \mathrm{C} & 2.13684 & -1.29345 & -1.3581 \\ \mathrm{C} & -1.24072 & -0.20719 & 0.19699 \\ \mathrm{O} & 2.56944 & -0.19455 & -1.74012 \\ \mathrm{~N} & -1.39084 & 0.37649 & 1.46375 \\ \mathrm{C} & -2.44886 & -0.76596 & -0.11778 \\ \mathrm{C} & -2.64641 & 0.16706 & 1.87873 \\ \mathrm{~N} & -3.29724 & -0.52671 & 0.94416 \\ \mathrm{~N} & 2.68508 & -2.45015 & -1.78272 \\ \mathrm{C} & 2.35231 & -3.8309 & -1.37132 \\ \mathrm{C} & 3.85779 & -2.39382 & -2.66748 \\ \mathrm{C} & 3.65086 & -4.59036 & -1.66217 \\ \mathrm{C} & 4.19731 & -3.88232 & -2.91178 \\ \mathrm{C} & -0.37615 & 1.07399 & 2.25794 \\ \mathrm{C} & 6.75107 & 0.14729 & -2.33476 \\ \mathrm{~N} & 5.64179 & -0.72756 & -2.7452 \\ \mathrm{C} & 6.32925 & 1.21369 & -1.2816 \\ \mathrm{O} & 7.19088 & 1.89758 & -0.74005 \\ \mathrm{C} & 7.19486 & 0.92939 & -3.58673 \\ \mathrm{C} & 7.93041 & -0.66835 & -1.77888 \\ \mathrm{C} & 1.34644 & -2.33668 & 1.88283 \\ \mathrm{O} & 0.8041 & -3.40346 & 1.62878 \\ \mathrm{O} & 1.87798 & -1.97828 & 3.08051 \\ \mathrm{C} & 1.88048 & -2.92247 & 4.21819 \\ \mathrm{C} & 2.68015 & -4.17812 & 3.85897 \\ \mathrm{C} & 2.58944 & -2.12324 & 5.31365 \\ \mathrm{C} & 0.44035 & -3.24561 & 4.62801 \\ \mathrm{C} & 1.10256 & 5.54892 & 1.21296 \\ \mathrm{C} & 1.57987 & 4.52204 & 0.39703 \\ \mathrm{C} & 1.98519 & 6.49495 & 1.73465 \\ \mathrm{C} & 2.94071 & 4.42373 & 0.08162 \\ \mathrm{C} & 3.34456 & 6.41026 & 1.42763 \\ \mathrm{C} & 3.45108 & 3.31439 & -0.81627\end{array}$




\begin{tabular}{|c|c|c|c|}
\hline $\mathrm{C}$ & 3.81592 & 5.38599 & 0.60661 \\
\hline $\mathrm{C}$ & 4.50273 & 2.40741 & -0.15275 \\
\hline $\mathrm{N}$ & 4.99221 & 1.39994 & +-1.0853 \\
\hline $\mathrm{C}$ & 3.94713 & 1.68449 & 1.07745 \\
\hline $\mathrm{O}$ & 2.78892 & 1.31483 & 1.2071 \\
\hline $\mathrm{O}$ & $4.9 \quad 1$ & $1.46039 \quad 1$ & 1.98296 \\
\hline $\mathrm{C}$ & 4.51781 & 0.70835 & 3.15075 \\
\hline $\mathrm{O}$ & 5.32477 & -1.9127 & -0.80741 \\
\hline $\mathrm{C}$ & 5.01186 & -1.65004 & +1.96286 \\
\hline $\mathrm{C}$ & -5.61583 & 2.3515 & -1.59557 \\
\hline $\mathrm{C}$ & -6.46436 & 3.62899 & -1.54943 \\
\hline $\mathrm{C}$ & -6.01034 & 1.33072 & -0.51246 \\
\hline $\mathrm{C}$ & -7.96044 & 3.29728 & -1.62824 \\
\hline $\mathrm{C}$ & -7.52015 & 1.01471 & -0.57866 \\
\hline $\mathrm{C}$ & -8.35941 & 2.30759 & -0.52699 \\
\hline $\mathrm{O}$ & -5.19635 & 0.18069 & $9-0.62714$ \\
\hline $\mathrm{N}$ & -7.88049 & 0.10816 & $6 \quad 0.51065$ \\
\hline $\mathrm{C}$ & -9.04424 & -0.59703 & $\begin{array}{ll}3 & 0.52299\end{array}$ \\
\hline $\mathrm{O}$ & -9.89067 & $7-0.51801$ & $1-0.36407$ \\
\hline $\mathrm{C}$ & -9.24263 & -1.50103 & $\begin{array}{ll}3 & 1.73546\end{array}$ \\
\hline $\mathrm{C}$ & -4.7444 & -0.88753 & 1.09621 \\
\hline $\mathrm{C}$ & -5.10917 & -2.21865 & $\begin{array}{ll}5 & 0.49801\end{array}$ \\
\hline $\mathrm{O}$ & -5.30923 & $3-0.34652$ & $2 \quad 2.0335$ \\
\hline $\mathrm{C}$ & -4.54601 & -1.67618 & $8-3.20748$ \\
\hline $\mathrm{O}$ & -3.57112 & -2.07936 & $6-2.53255$ \\
\hline $\mathrm{O}$ & -5.45251 & -0.85801 & $1-2.81511$ \\
\hline $\mathrm{C}$ & -4.7244 & -2.19152 & -4.63307 \\
\hline $\mathrm{H}$ & 2.63022 & -2.70905 & $5 \quad 6.23632$ \\
\hline $\mathrm{H}$ & 3.614 & -1.88002 & 5.01743 \\
\hline $\mathrm{H}$ & 2.05566 & -1.19121 & $1 \quad 5.52145$ \\
\hline $\mathrm{H}$ & 3.6807 & -3.90545 & 3.5093 \\
\hline $\mathrm{H}$ & 2.79103 & -4.80545 & $5 \quad 4.749$ \\
\hline $\mathrm{H}$ & 2.17785 & -4.75552 & 23.08313 \\
\hline $\mathrm{H}$ & -0.10723 & $3-2.32569$ & $9 \quad 4.85771$ \\
\hline $\mathrm{H}$ & -0.08117 & $7-3.78186$ & $\begin{array}{ll}6 & 3.83523\end{array}$ \\
\hline $\mathrm{H}$ & 0.44617 & -3.86671 & $1 \quad 5.52917$ \\
\hline $\mathrm{H}$ & 2.03426 & -0.49192 & $2 \quad 1.27024$ \\
\hline $\mathrm{H}$ & 0.42021 & -2.29751 & $1-0.44841$ \\
\hline $\mathrm{H}$ & 0.5623 & 0.75579 & -0.4923 \\
\hline $\mathrm{H}$ & -0.29517 & $7-0.20538$ & $8-1.67994$ \\
\hline $\mathrm{H}$ & 0.22614 & 0.35764 & 2.81919 \\
\hline $\mathrm{H}$ & -0.88119 & 1.75513 & 32.94404 \\
\hline $\mathrm{H}$ & 0.28334 & 1.64512 & 1.60614 \\
\hline $\mathrm{H}$ & -3.08541 & 0.50209 & $\begin{array}{l}9 \\
2.80379\end{array}$ \\
\hline $\mathrm{H}$ & -2.7996 & -1.25866 & -1.03165 \\
\hline $\mathrm{H}$ & -6.18963 & $3-2.23898$ & $\begin{array}{ll}8 & 0.35953\end{array}$ \\
\hline $\mathrm{H}$ & -4.82908 & 3 -2.99591 & $1 \quad 1.22025$ \\
\hline $\mathrm{H}$ & -4.61612 & -2.41001 & $1 \quad-0.45633$ \\
\hline $\mathrm{H}$ & -4.75112 & $2-1.34833$ & $\begin{array}{lll}3 & -5.33047\end{array}$ \\
\hline $\mathrm{H}$ & -5.68676 & $5-2.70578$ & $8 \begin{array}{ll}8 & -4.71921\end{array}$ \\
\hline $\mathrm{H}$ & -3.91679 & -2.87297 & 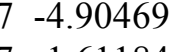 \\
\hline $\mathrm{H}$ & -5.30663 & $3-0.33217$ & $7-1.61184$ \\
\hline $\mathrm{H}$ & -5.80736 & $5 \quad 1.76768$ & 80.48103 \\
\hline $\mathrm{H}$ & -4.55016 & $5 \quad 2.58026$ & $6-1.47857$ \\
\hline
\end{tabular}

$\begin{array}{llll}\mathrm{H} & -5.72601 & 1.86227 & -2.57145 \\ \mathrm{H} & -6.1725 & 4.3011 & -2.36566 \\ \mathrm{H} & -6.2639 & 4.17078 & -0.61341 \\ \mathrm{H} & -8.56185 & 4.21084 & -1.54901 \\ \mathrm{H} & -8.18458 & 2.85993 & -2.61089 \\ \mathrm{H} & -9.41428 & 2.0316 & -0.60647 \\ \mathrm{H} & -8.21919 & 2.77574 & 0.45825 \\ \mathrm{H} & -7.72562 & 0.498 & -1.52467 \\ \mathrm{H} & -7.21339 & -0.0133 & 1.26355 \\ \mathrm{H} & -8.36801 & -1.55699 & 2.3896 \\ \mathrm{H} & -9.49691 & -2.50438 & 1.38394 \\ \mathrm{H} & -10.09593 & -1.13477 & 2.31426 \\ \mathrm{H} & 2.05055 & -3.87453 & -0.32484 \\ \mathrm{H} & 1.5246 & -4.21254 & -1.98404 \\ \mathrm{H} & 3.48008 & -5.65882 & -1.81548 \\ \mathrm{H} & 4.34688 & -4.45732 & -0.83004 \\ \mathrm{H} & 5.26802 & -4.03935 & -3.06292 \\ \mathrm{H} & 3.6765 & -4.22956 & -3.81052 \\ \mathrm{H} & 3.58825 & -1.87702 & -3.59501 \\ \mathrm{H} & 5.21073 & -0.51559 & -3.63351 \\ \mathrm{H} & 8.31624 & -1.33372 & -2.5573 \\ \mathrm{H} & 8.71842 & 0.01696 & -1.46419 \\ \mathrm{H} & 7.61607 & -1.26718 & -0.9243 \\ \mathrm{H} & 7.99174 & 1.6231 & -3.315 \\ \mathrm{H} & 7.56757 & 0.24191 & -4.3525 \\ \mathrm{H} & 6.36617 & 1.51138 & -4.00693 \\ \mathrm{H} & 4.30543 & 0.77036 & -1.49276 \\ \mathrm{H} & 5.37988 & 2.97678 & 0.16077 \\ \mathrm{H} & 2.6192 & 2.69173 & -1.15834 \\ \mathrm{H} & 3.92371 & 3.7396 & -1.7095 \\ \mathrm{H} & 4.16389 & -0.2861 & 2.8717 \\ \mathrm{H} & 3.72862 & 1.23121 & 3.696 \\ \mathrm{H} & 5.42054 & 0.6384 & 3.75571 \\ \mathrm{H} & 4.87501 & 5.34036 & 0.36478 \\ \mathrm{H} & 4.03873 & 7.14642 & 1.82262 \\ \mathrm{H} & 1.61711 & 7.29532 & 2.36967 \\ \mathrm{H} & 0.04136 & 5.61223 & 1.43694 \\ \mathrm{H} & 0.88665 & 3.79318 & -0.01487\end{array}$




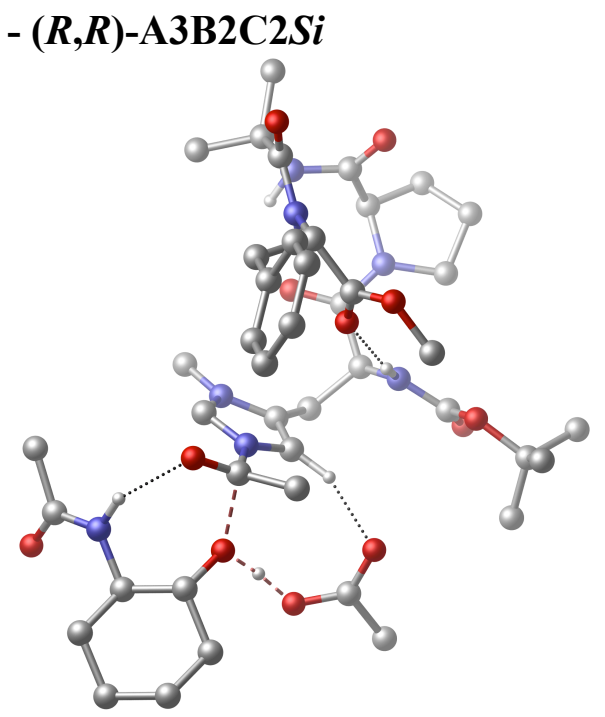

$\begin{array}{llll}\mathrm{N} & -1.58955 & 2.46964 & -0.43011\end{array}$

$\begin{array}{lllll}\text { C } & -1.74441 & 2.21374 & -1.84301\end{array}$

$\begin{array}{lllll}\text { C } & & -0.39586 & 1.88626 & -2.55009\end{array}$

$\begin{array}{lllll}\text { C } & -2.84395 & 1.17434 & -2.13805\end{array}$

$\begin{array}{lllll}\text { C } & 0.45695 & 0.8463 & -1.88932\end{array}$

$\begin{array}{lllll}\mathrm{O} & -2.60394 & 0.1587 & -2.81521\end{array}$

$\begin{array}{lllll}\mathrm{N} & 0.64028 & -0.45874 & -2.35649\end{array}$

$\begin{array}{lllll}\text { C } & 1.20473 & 0.95038 & -0.74624\end{array}$

$\begin{array}{llll}\text { C } & 1.4497 & -1.10523 & -1.51223\end{array}$

$\mathrm{N} \quad 1.7969-0.27716-0.52661$

$\begin{array}{lllll}\mathrm{N} & & -4.09878 & 1.44117 & -1.70601\end{array}$

$\begin{array}{llll}\text { C } & -4.55753 & 2.6199 & -0.93358\end{array}$

$\begin{array}{lllll}\text { C } & & -5.23173 & 0.63247 & -2.21482\end{array}$

$\begin{array}{lllll}\text { C } & -6.07491 & 2.41417 & -0.81769\end{array}$

$\begin{array}{lllll}\text { C } & -6.43624 & 1.56941 & -2.04799\end{array}$

$\begin{array}{llll}\text { C } & 0.14273 & -1.02188 & -3.6128\end{array}$

$\begin{array}{lllll}\text { C } & -4.7458 & -3.10711 & -1.40438\end{array}$

$\mathrm{N} \quad \begin{array}{llll}\mathrm{C} & -4.71485 & -1.7362 & -1.93823\end{array}$

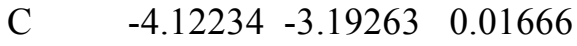

$\begin{array}{lllll}\mathrm{O} & -4.44224 & -4.07424 & 0.80661\end{array}$

$\begin{array}{llll}\text { C } & -3.86914 & -3.97381 & -2.33059\end{array}$

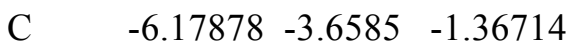

$\begin{array}{llll}\text { C } & -1.51634 & 3.75777 & 0.03923\end{array}$

$\begin{array}{lllll}\text { O } & -1.66166 & 4.74406 & -0.66874\end{array}$

$\begin{array}{lllll}\text { O } & -1.30486 & 3.74334 & 1.37068\end{array}$

$\begin{array}{llll}\text { C } & -1.02847 & 4.99976 & 2.1078\end{array}$

$\begin{array}{llll}\text { C } & -2.28634 & 5.87205 & 2.11162\end{array}$

$\begin{array}{llll}\text { C } & -0.70552 & 4.49194 & 3.51437\end{array}$

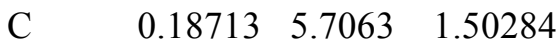

$\begin{array}{llll}\mathrm{C} & 1.43612 & -3.39226 & 4.2436\end{array}$

$\begin{array}{llll}\text { C } & 0.77862 & -3.23739 & 3.02069\end{array}$

$\begin{array}{llll}\text { C } & 0.73685 & -3.83612 & 5.36546\end{array}$

$\begin{array}{llll}\text { C } & -0.58707 & -3.52527 & 2.90388\end{array}$

$\begin{array}{llll}\text { C } & -0.62358 & -4.13456 & 5.25647\end{array}$

$\begin{array}{llll}\text { C } & -1.30135 & -3.36749 & 1.57977\end{array}$

$\begin{array}{llll}\text { C } & -1.27812 & -3.98363 & 4.03523\end{array}$

C

C

$\mathrm{O}$

$\mathrm{O}$

C

$\mathrm{O}$

C

C

C

$\mathrm{O}$

C

C

C

C

C

C

O

$\mathrm{N}$

C

O

C

C

O

O

C

$\mathrm{H}$

$\mathrm{H}$

$\mathrm{H}$

$\mathrm{H}$

H

$\mathrm{H}$

$\mathrm{H}$

$\mathrm{H}$

$\mathrm{H}$

H

$\mathrm{H}$

$\mathrm{H}$

$\mathrm{H}$

$\mathrm{H}$

$\mathrm{H}$

$\mathrm{H}$

$\mathrm{H}$

H

$\mathrm{H}$

$\mathrm{H}$

H

$\mathrm{H}$

$\mathrm{H}$

$\mathrm{H}$

$\mathrm{H}$

$\mathrm{H}$

$\mathrm{H}$

H $\begin{array}{llll}-2.43842 & -2.2998 & 1.55681\end{array}$

$\begin{array}{llll}-3.13031 & -2.29942 & 0.28062\end{array}$

$\begin{array}{lll}-1.89686 & -0.89796 & 1.7809\end{array}$

$\begin{array}{lll}-1.54403 & -0.16804 & 0.86475\end{array}$

$\begin{array}{lll}-1.83741 & -0.57257 & 3.06708\end{array}$

$\begin{array}{lll}-1.28663 & 0.72551 & 3.38469\end{array}$

$\begin{array}{lll}-6.11161 & -0.74644 & -0.4157\end{array}$

$\begin{array}{llll}-5.40241 & -0.68229 & -1.41402\end{array}$

$\begin{array}{llll}2.74751 & -0.71417 & 0.55543\end{array}$

$\begin{array}{llll}2.63519 & 0.07927 & 1.83171\end{array}$

$\begin{array}{llll}3.05014 & -1.90506 & 0.52006\end{array}$

$\begin{array}{llll}8.42261 & -0.47689 & -0.6591\end{array}$

$\begin{array}{llll}7.92646 & 0.21294 & 0.61854\end{array}$

$\begin{array}{llll}7.43559 & -1.55874 & -1.11202\end{array}$

$\begin{array}{llll}6.52006 & 0.79521 & 0.41965\end{array}$

$\begin{array}{llll}6.01361 & -0.99712 & -1.31275\end{array}$

$\begin{array}{llll}5.5142 & -0.2686 & -0.04818\end{array}$

$\begin{array}{llll}4.22395 & 0.28572 & -0.27447\end{array}$

$\begin{array}{llll}5.07387 & -2.0634 & -1.65338\end{array}$

$\begin{array}{llll}5.01521 & -2.62253 & -2.88879\end{array}$

$\begin{array}{llll}5.7378 & -2.28636 & -3.82411\end{array}$

$\begin{array}{lll}3.50655 & 3.5171 & 0.12181\end{array}$

$\begin{array}{llll}3.88203 & 4.99042 & 0.00329\end{array}$

$\begin{array}{llll}2.49728 & 3.20667 & 0.79753\end{array}$

$\begin{array}{llll}4.27195 & 2.70073 & -0.50529\end{array}$

$\begin{array}{llll}3.97465 & -3.72847 & -3.05475\end{array}$

$\begin{array}{lll}-1.55141 & 3.93928 & 3.93525\end{array}$

$\begin{array}{llll}-0.48973 & 5.33738 & 4.17382\end{array}$

$\begin{array}{lll}0.17211 & 3.84005 & 3.49127\end{array}$

$\begin{array}{llll}-3.13653 & 5.32008 & 2.52594\end{array}$

$\begin{array}{llll}-2.11789 & 6.75398 & 2.73792\end{array}$

$\begin{array}{llll}-2.53305 & 6.20188 & 1.10196\end{array}$

$\begin{array}{lll}0.49352 & 6.52223 & 2.16609\end{array}$

$\begin{array}{llll}-0.04886 & 6.12557 & 0.52414\end{array}$

$\begin{array}{lll}1.01947 & 5.00234 & 1.40129\end{array}$

$\begin{array}{lll}-1.40894 & 1.69524 & 0.20125\end{array}$

$\begin{array}{llll}-2.08493 & 3.16342 & -2.27285\end{array}$

$\begin{array}{llll}-0.59402 & 1.62195 & -3.59106\end{array}$

$\begin{array}{llll}0.17055 & 2.82232 & -2.55613\end{array}$

$\begin{array}{llll}1.42183 & 1.80921 & -0.11482\end{array}$

$\begin{array}{llll}0.6821 & -0.57902 & -4.45418\end{array}$

$\begin{array}{llll}0.32206 & -2.09747 & -3.60414\end{array}$

$-0.92304-0.81405-3.6909$

$\begin{array}{llll}1.79136 & -2.1214 & -1.60421\end{array}$

$\begin{array}{llll}2.56026 & 1.15619 & 1.66903\end{array}$

$\begin{array}{lll}1.75924 & -0.28834 & 2.38012\end{array}$

$\begin{array}{llll}3.51906 & -0.14186 & 2.43211\end{array}$

$\begin{array}{llll}3.04955 & 5.54597 & -0.43966\end{array}$

$\begin{array}{lll}4.04622 & 5.40334 & 1.00349\end{array}$

$\begin{array}{llll}4.7786 & 5.126 & -0.60255\end{array}$

$\begin{array}{llll}4.19165 & 1.42131 & -0.33852\end{array}$

$\begin{array}{llll}6.55072 & 1.60091 & -0.32246\end{array}$

$\begin{array}{lll}6.14921 & 1.24661 & 1.34665\end{array}$

$\begin{array}{lll}8.61624 & 1.00925 & 0.92203\end{array}$ 


$\begin{array}{llll}\mathrm{H} & 7.91189 & -0.51707 & 1.4407 \\ \mathrm{H} & 8.53862 & 0.2717 & -1.45507 \\ \mathrm{H} & 9.41387 & -0.9171 & -0.49888 \\ \mathrm{H} & 7.39043 & -2.35542 & -0.356 \\ \mathrm{H} & 7.75666 & -2.02169 & -2.04942 \\ \mathrm{H} & 6.02763 & -0.28184 & -2.14434 \\ \mathrm{H} & 5.41279 & -1.02963 & 0.74428 \\ \mathrm{H} & 4.40835 & -2.33656 & -0.93798 \\ \mathrm{H} & 3.41969 & -3.95629 & -2.13921 \\ \mathrm{H} & 4.48212 & -4.63759 & -3.38833 \\ \mathrm{H} & 3.27717 & -3.44486 & -3.8492 \\ \mathrm{H} & -4.0684 & 2.64992 & 0.04024 \\ \mathrm{H} & -4.30296 & 3.54345 & -1.46675 \\ \mathrm{H} & -6.61071 & 3.36577 & -0.77757 \\ \mathrm{H} & -6.30631 & 1.84395 & 0.08465 \\ \mathrm{H} & -7.36494 & 1.01286 & -1.91455 \\ \mathrm{H} & -6.52894 & 2.19798 & -2.9402 \\ \mathrm{H} & -5.03081 & 0.38862 & -3.26179 \\ \mathrm{H} & -4.0342 & -1.50557 & -2.65369 \\ \mathrm{H} & -2.84242 & -3.59482 & -2.37744 \\ \mathrm{H} & -4.28558 & -3.98784 & -3.34274 \\ \mathrm{H} & -3.83744 & -4.99841 & -1.95392 \\ \mathrm{H} & -6.58277 & -3.69832 & -2.38311 \\ \mathrm{H} & -6.16906 & -4.66189 & -0.93967 \\ \mathrm{H} & -6.81749 & -3.02564 & -0.75138 \\ \mathrm{H} & -2.90284 & -1.56586 & -0.37619 \\ \mathrm{H} & -3.15719 & -2.53806 & 2.34426 \\ \mathrm{H} & -1.84616 & 1.51521 & 2.88153 \\ \mathrm{H} & -1.37282 & 0.81464 & 4.46609 \\ \mathrm{H} & -0.24041 & 0.77925 & 3.07851 \\ \mathrm{H} & -1.79228 & -4.30539 & 1.30012 \\ \mathrm{H} & -0.58595 & -3.12007 & 0.78805 \\ \mathrm{H} & -2.33344 & -4.23324 & 3.95576 \\ \mathrm{H} & -1.17375 & -4.49227 & 6.12213 \\ \mathrm{H} & 1.24784 & -3.9578 & 6.31612 \\ \mathrm{H} & 2.49732 & -3.17101 & 4.31233 \\ \mathrm{H} & 1.34312 & -2.9115 & 2.15027\end{array}$

\section{- $(S, S)-\mathrm{A} 3 \mathrm{~B} 2 \mathrm{C} 2 S i$}

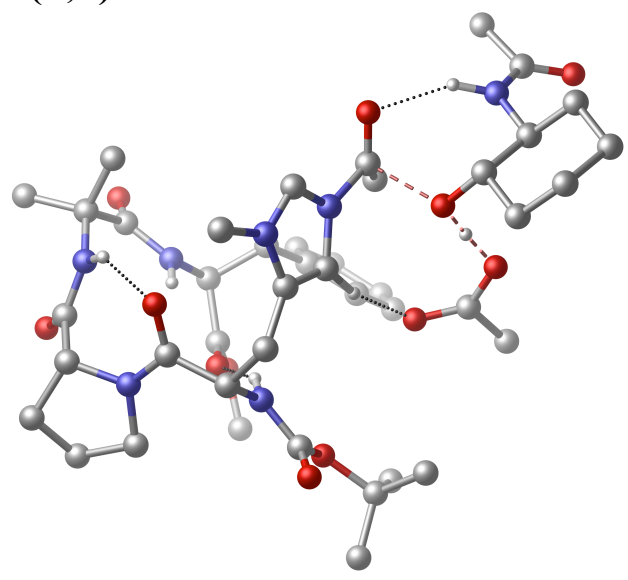

$\begin{array}{llll}-1.72699 & 0.04887 & -2.38401\end{array}$ $\begin{array}{llll}-1.93306 & -1.34923 & -2.70081\end{array}$ $\begin{array}{llll}-0.59766 & -2.11233 & -2.89362\end{array}$ $\begin{array}{llll}-2.8765 & -2.04888 & -1.70754\end{array}$ $0.28589-2.27024-1.69272$ $\begin{array}{llll}-2.50533 & -3.06933 & -1.09862\end{array}$ $\begin{array}{llll}0.57067 & -3.52242 & -1.13421\end{array}$ $\begin{array}{llll}1.02123 & -1.35773 & -0.97891\end{array}$ $\begin{array}{llll}1.43512 & -3.35981 & -0.12914\end{array}$ $\begin{array}{llll}1.7151 & -2.06344 & -0.00903\end{array}$ $\begin{array}{llll}-4.12799 & -1.55944 & -1.56208\end{array}$ $\begin{array}{llll}-4.73411 & -0.42435 & -2.30026\end{array}$ $\begin{array}{llll}-5.14339 & -2.32805 & -0.79751\end{array}$ $-6.18422-0.39868-1.79766$ $\begin{array}{llll}-6.47035 & -1.85392 & -1.39984\end{array}$ $\begin{array}{llll}0.03009 & -4.8132 & -1.55632\end{array}$ $\begin{array}{lll}-3.7486 & -2.55621 & 2.79783\end{array}$ $\begin{array}{lll}-4.02705 & -2.65781 & 1.354\end{array}$ $\begin{array}{lll}-3.41317 & -1.09488 \quad 3.2166\end{array}$ $\begin{array}{llll}-3.58158 & -0.70032 & 4.36322\end{array}$ $\begin{array}{llll}-2.49391 & -3.40565 & 3.07932\end{array}$ $\begin{array}{llll}-4.93352 & -3.06378 & 3.63294\end{array}$ $\begin{array}{llll}-1.43327 & 0.91955 & -3.40354\end{array}$ $\begin{array}{llll}-1.2792 & 0.55496 & -4.56264\end{array}$ $\begin{array}{llll}-1.36136 & 2.17658 & -2.9354\end{array}$ $\begin{array}{llll}-0.74158 & 3.24815 & -3.74713\end{array}$ $\begin{array}{llll}-1.65257 & 3.58491 & -4.92965\end{array}$ $\begin{array}{llll}-0.66414 & 4.40451 & -2.74929\end{array}$ $\begin{array}{llll}0.66464 & 2.82445 & -4.18084\end{array}$ $\begin{array}{llll}0.46576 & 4.77253 & 2.09366\end{array}$ $\begin{array}{llll}0.10616 & 3.43409 & 1.91802\end{array}$ $\begin{array}{llll}0.07441 & 5.46331 & 3.24059\end{array}$ $\begin{array}{lll}-0.64886 & 2.77075 & 2.89483\end{array}$ $\begin{array}{lll}-0.6748 & 4.8074 & 4.22005\end{array}$ $\begin{array}{llll}-1.0445 & 1.32261 & 2.71022\end{array}$ $\begin{array}{llll}-1.03034 & 3.47063 & 4.04827\end{array}$ $\begin{array}{llll}-2.55435 & 1.07463 & 2.41211\end{array}$ $\begin{array}{llll}-2.82647 & -0.34074 & 2.24581\end{array}$ $\begin{array}{lll}-2.97457 & 1.76414 & 1.12323\end{array}$ $\begin{array}{lll}-2.93888 & 1.214 & 0.03119\end{array}$ $\begin{array}{lll}-3.35781 & 3.02124 & 1.30925\end{array}$ $\begin{array}{llll}-3.68221 & 3.77531 & 0.12085\end{array}$ $\begin{array}{llll}-5.85718 & -1.28623 & 1.28338\end{array}$ $\begin{array}{llll}-5.05782 & -2.02878 & 0.72347\end{array}$ $\begin{array}{llll}5.57168 & -1.34667 & -2.11953\end{array}$ $\begin{array}{llll}6.95287 & -1.9519 & -2.40303\end{array}$ $5.17666-1.43295-0.63478$ $8.02414-1.30938-1.51232$ $6.26293-0.80491 \quad 0.26425$ $7.64474-1.42498-0.03135$ $\begin{array}{llll}3.89566 & -0.84822 & -0.46324\end{array}$ $\begin{array}{llll}5.90084 & -0.97207 & 1.67061\end{array}$ $\begin{array}{llll}6.49029 & -0.25481 & 2.66662\end{array}$ $\begin{array}{llll}7.37279 & 0.57596 & 2.46696\end{array}$ 


\begin{tabular}{|c|c|c|c|}
\hline & 5.98267 & -0.55815 & 4.0723 \\
\hline & 2.726 & -1.60048 & 1.00998 \\
\hline & 2.39927 & -0.27296 & 1.63496 \\
\hline & 3.33462 & -2.50898 & 1.55779 \\
\hline & 2.92829 & 2.24505 & -0.90544 \\
\hline & 1.75626 & 1.78883 & -0.89207 \\
\hline & 4.00146 & 1.56366 & -0.76707 \\
\hline & 3.15419 & 3.74036 & -1.11638 \\
\hline & -0.17979 & 5.26888 & -3.21289 \\
\hline & -1.6642 & 4.70852 & -2.42425 \\
\hline & -0.0836 & 4.09883 & -1.8752 \\
\hline & -2.65615 & 3.84547 & -4.5779 \\
\hline & -1.24815 & 4.4468 & $-5.4702^{\prime}$ \\
\hline & -1.72496 & 2.74075 & -5.61632 \\
\hline & 1.22851 & 2.48529 & -3.30752 \\
\hline & 0.62813 & 2.02555 & -4.92229 \\
\hline & 1.18134 & 3.68356 & -4.62054 \\
\hline & -1.87113 & 0.41787 & -1.4505 \\
\hline & -2.41913 & -1.3767 & -3.6858 \\
\hline & -0.82018 & -3.09578 & -3.3149 \\
\hline & -0.06082 & -1.55457 & -3.666 \\
\hline & -1.05804 & -4.7604 & -1.5345 \\
\hline & 0.38341 & -5.57878 & -0.865 \\
\hline & 0.38064 & -5.0568 & -2.56218 \\
\hline & 1.86399 & -4.1308 & 0.48892 \\
\hline & 1.1605 & -0.28561 & $-1.1007^{7}$ \\
\hline & 3.30997 & 0.12259 & 2.0836 \\
\hline & 1.66462 & -0.45254 & 2.4290 \\
\hline & 2.00701 & 0.4501 & 0.91873 \\
\hline & 3.53175 & 3.90609 & -2.1314 \\
\hline & 3.91853 & 4.10433 & -0.4252 \\
\hline & 2.22903 & 4.30526 & -0.9902 \\
\hline & 3.90669 & 0.25156 & -0.5944 \\
\hline & 5.07706 & -2.49339 & -0.3484 \\
\hline & 4.79261 & -1.83989 & -2.7119 \\
\hline & 5.56253 & -0.28749 & -2.4065 \\
\hline & 7.20394 & -1.82938 & -3.4635 \\
\hline & 6.92573 & -3.03464 & -2.2120 \\
\hline & 9.00076 & -1.77594 & -1.6880 \\
\hline & 8.13184 & -0.24939 & -1.7803 \\
\hline & 8.38128 & -0.92817 & 0.6054 \\
\hline & 7.62222 & -2.48443 & 0.2623 \\
\hline & 6.30794 & 0.27162 & 0.0581 \\
\hline & 5.17522 & -1.64298 & 1.8958 \\
\hline & 5.68523 & 0.38114 & 4.54603 \\
\hline & 6.80456 & -0.97276 & 4.6634 \\
\hline & 5.14131 & -1.25642 & 4.0943 \\
\hline & -4.20024 & 0.50028 & -2.0845 \\
\hline & -4.6818 & -0.61698 & -3.3793 \\
\hline & -6.8702 & -0.01642 & -2.5576 \\
\hline & -6.25979 & 0.23507 & -0.9106 \\
\hline & -7.28531 & -1.93787 & -0.6802 \\
\hline & -6.70759 & -2.46043 & -2.280 \\
\hline & -4.95335 & -3.39338 & -0.957 \\
\hline
\end{tabular}

$\begin{array}{llll}\mathrm{H} & -3.3843 & -3.17343 & 0.75904 \\ \mathrm{H} & -5.10518 & -4.12374 & 3.42239 \\ \mathrm{H} & -4.71183 & -2.93464 & 4.69318 \\ \mathrm{H} & -5.83628 & -2.49996 & 3.3973 \\ \mathrm{H} & -2.22558 & -3.32409 & 4.13477 \\ \mathrm{H} & -2.68895 & -4.45876 & 2.85149 \\ \mathrm{H} & -1.64223 & -3.06615 & 2.48064 \\ \mathrm{H} & -2.83262 & -0.68179 & 1.2953 \\ \mathrm{H} & -3.15524 & 1.44805 & 3.24436 \\ \mathrm{H} & -0.46664 & 0.87686 & 1.89466 \\ \mathrm{H} & -0.83074 & 0.74578 & 3.61688 \\ \mathrm{H} & -4.6534 & 3.45541 & -0.26497 \\ \mathrm{H} & -2.91869 & 3.62568 & -0.64326 \\ \mathrm{H} & -3.72392 & 4.81507 & 0.44076 \\ \mathrm{H} & -1.60391 & 2.96238 & 4.82012 \\ \mathrm{H} & -0.97558 & 5.33474 & 5.12091 \\ \mathrm{H} & 0.35865 & 6.50285 & 3.37656 \\ \mathrm{H} & 1.06173 & 5.271 & 1.33449 \\ \mathrm{H} & 0.4333 & 2.90269 & 1.02585\end{array}$

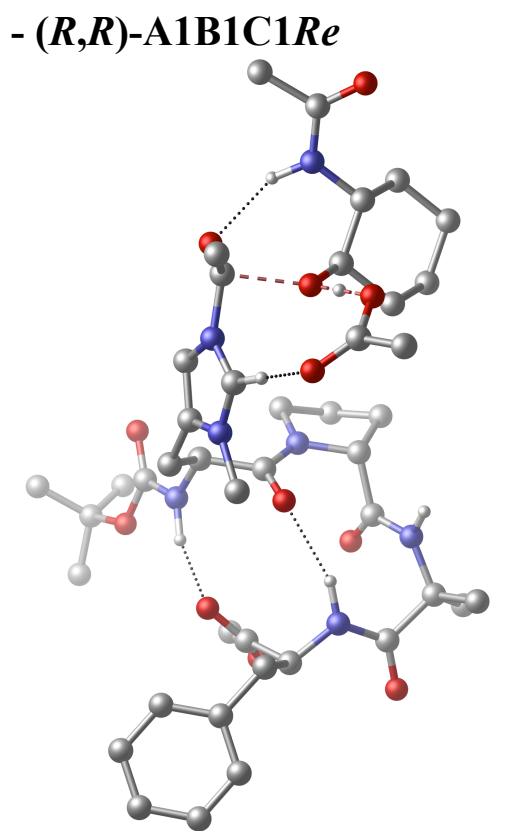

$\begin{array}{lcll}\mathrm{N} & 2.17009 & 2.33894 & -0.71248 \\ \mathrm{C} & 0.83955 & 1.76313 & -0.76531 \\ \mathrm{C} & 0.57858 & 1.07721 & -2.12723 \\ \mathrm{C} & 0.66803 & 0.75696 & 0.39095 \\ \mathrm{C} & -0.82372 & 0.5639 & -2.26109 \\ \mathrm{O} & 0.8917 & -0.45416 & 0.22509 \\ \mathrm{~N} & -1.15901 & -0.78941 & -2.37908 \\ \mathrm{C} & -2.01309 & 1.23891 & -2.25906 \\ \mathrm{C} & -2.48946 & -0.91516 & -2.44256 \\ \mathrm{~N} & -3.02399 & 0.3077 & -2.38115 \\ \mathrm{~N} & 0.28449 & 1.24 & 1.58955 \\ \mathrm{C} & 0.05526 & 2.64781 & 1.97347 \\ \mathrm{C} & 0.12812 & 0.31569 & 2.72098\end{array}$ 


\begin{tabular}{|c|c|c|}
\hline $\mathrm{C}$ & $0.22847 \quad 2.61288$ & 3.49492 \\
\hline $\mathrm{C}$ & $\begin{array}{ll}-0.33355 & 1.23448\end{array}$ & 3.87694 \\
\hline $\mathrm{C}$ & $\begin{array}{ll}-0.23867 & -1.92809\end{array}$ & -2.40029 \\
\hline $\mathrm{C}$ & $2.48467-2.60872$ & 3.59953 \\
\hline $\mathrm{N}$ & $1.37447-1.71887$ & 3.22527 \\
\hline $\mathrm{C}$ & $3.61655-2.62895$ & 2.53373 \\
\hline $\mathrm{O}$ & $\begin{array}{lll}4.71992 & -3.06851\end{array}$ & 2.83052 \\
\hline $\mathrm{C}$ & $1.91769-4.03957$ & 3.67252 \\
\hline $\mathrm{C}$ & $3.08184-2.20036$ & 4.9562 \\
\hline $\mathrm{C}$ & $2.36197 \quad 3.69231$ & -0.71607 \\
\hline $\mathrm{O}$ & $1.45663 \quad 4.5155$ & -0.65514 \\
\hline $\mathrm{O}$ & $3.68577 \quad 3.96318$ & -0.7756 \\
\hline $\mathrm{C}$ & $4.17449 \quad 5.35604$ & -0.80192 \\
\hline $\mathrm{C}$ & $3.77149 \quad 6.07918$ & 0.48681 \\
\hline $\mathrm{C}$ & $5.69209 \quad 5.17329$ & -0.87137 \\
\hline $\mathrm{C}$ & $3.65491 \quad 6.06558$ & -2.05594 \\
\hline $\mathrm{C}$ & $6.29158-2.05663$ & -3.9784 \\
\hline $\mathrm{C}$ & $5.22561-1.97258$ & -3.08127 \\
\hline $\mathrm{C}$ & $7.22628 \quad-3.0861$ & -3.86369 \\
\hline $\mathrm{C}$ & $5.07456-2.91709$ & -2.05749 \\
\hline $\mathrm{C}$ & $7.08838-4.03162$ & -2.84584 \\
\hline $\mathrm{C}$ & $3.93106-2.82966$ & -1.06632 \\
\hline $\mathrm{C}$ & $6.02108-3.9462$ & -1.95234 \\
\hline $\mathrm{C}$ & $4.31192-2.10866$ & 0.23818 \\
\hline $\mathrm{N}$ & $3.28 \quad-2.24277$ & 1.26498 \\
\hline $\mathrm{C}$ & $4.51793-0.60524$ & 0.04131 \\
\hline $\mathrm{O}$ & $3.93596 \quad 0.06091$ & -0.80211 \\
\hline $\mathrm{O}$ & $5.33614-0.10443$ & 0.96451 \\
\hline $\mathrm{C}$ & $5.39837 \quad 1.33224$ & 1.07251 \\
\hline $\mathrm{O}$ & $2.51056 \quad 0.26637$ & 3.14362 \\
\hline $\mathrm{C}$ & $1.4679 \quad-0.3728$ & 3.04102 \\
\hline $\mathrm{C}$ & $-4.48382 \quad 0.64312$ & -2.28914 \\
\hline $\mathrm{C}$ & $\begin{array}{ll}-5.35618 & -0.17549\end{array}$ & -3.20865 \\
\hline $\mathrm{O}$ & $-4.72206 \quad 1.78804$ & -1.93282 \\
\hline $\mathrm{C}$ & $-5.99004 \quad 0.04024$ & 3.33632 \\
\hline $\mathrm{C}$ & $-4.49312 \quad-0.13484$ & 3.04628 \\
\hline $\mathrm{C}$ & $-6.66943 \quad 0.85781$ & 2.23104 \\
\hline $\mathrm{C}$ & $-4.27226 \quad-0.75679$ & 1.66049 \\
\hline $\mathrm{C}$ & $\begin{array}{ll}-6.4633 & 0.22857\end{array}$ & 0.83777 \\
\hline $\mathrm{C}$ & $-4.96191 \quad 0.0339$ & 0.53244 \\
\hline $\mathrm{O}$ & $-4.72824-0.59532$ & -0.70542 \\
\hline $\mathrm{N}$ & $\begin{array}{ll}-7.06991 & 1.04844\end{array}$ & -0.20812 \\
\hline $\mathrm{C}$ & $-8.4156 \quad 1.11046$ & -0.39731 \\
\hline $\mathrm{O}$ & $-9.22265 \quad 0.50051$ & 0.30076 \\
\hline $\mathrm{C}$ & $-4.87215-3.70385$ & -1.54231 \\
\hline $\mathrm{C}$ & $-5.34792-5.14504$ & -1.44024 \\
\hline $\mathrm{O}$ & $\begin{array}{ll}-3.96119 & -3.40898\end{array}$ & -2.34357 \\
\hline $\mathrm{O}$ & $-5.49528-2.88183$ & $3-0.76806$ \\
\hline $\mathrm{C}$ & $-8.87011 \quad 2.00558$ & -1.54513 \\
\hline $\mathrm{H}$ & $6.05949 \quad 4.63824$ & 0.00922 \\
\hline $\mathrm{H}$ & $6.18523 \quad 6.14883$ & -0.91082 \\
\hline $\mathrm{H}$ & $5.97322 \quad 4.60697$ & -1.76373 \\
\hline $\mathrm{H}$ & $4.11176 \quad 5.51568$ & 1.3614 \\
\hline $\mathrm{H}$ & $4.24453 \quad 7.06584$ & 0.51557 \\
\hline
\end{tabular}

\begin{tabular}{|c|c|c|c|}
\hline $\mathrm{H}$ & 2.69043 & 6.20572 & 0.54406 \\
\hline $\mathrm{H}$ & 4.10393 & 7.06134 & -2.12645 \\
\hline $\mathrm{H}$ & 2.57038 & 6.17118 & -2.0247 \\
\hline $\mathrm{H}$ & 3.93343 & 5.50408 & -2.9532 \\
\hline $\mathrm{H}$ & 2.97247 & 1.71636 & -0.79023 \\
\hline $\mathrm{H}$ & 0.14248 & 2.59488 & -0.64619 \\
\hline $\mathrm{H}$ & 0.78092 & 1.8174 & -2.90767 \\
\hline $\mathrm{H}$ & 1.29841 & 0.26575 & -2.24916 \\
\hline $\mathrm{H}$ & -2.24226 & 2.28875 & -2.17845 \\
\hline $\mathrm{H}$ & 0.39868 & -1.87987 & -1.51717 \\
\hline $\mathrm{H}$ & -0.83593 & -2.84014 & -2.39078 \\
\hline $\mathrm{H}$ & 0.36956 & -1.90352 & -3.30788 \\
\hline $\mathrm{H}$ & -3.0503 & -1.86203 & -2.45053 \\
\hline $\mathrm{H}$ & -5.28633 & 0.2536 & -4.21554 \\
\hline $\mathrm{H}$ & -5.07357 & -1.22783 & -3.24261 \\
\hline $\mathrm{H}$ & -6.38448 & -0.09261 & -2.85844 \\
\hline $\mathrm{H}$ & -6.39612 & -5.20445 & -1.7498 \\
\hline $\mathrm{H}$ & -4.7421 & -5.79677 & -2.07064 \\
\hline $\mathrm{H}$ & -5.30392 & -5.47918 & -0.39951 \\
\hline $\mathrm{H}$ & -5.11448 & -1.72667 & -0.77569 \\
\hline $\mathrm{H}$ & -4.66484 & -1.78148 & 1.64563 \\
\hline $\mathrm{H}$ & -3.20339 & -0.82758 & 1.42165 \\
\hline $\mathrm{H}$ & -4.01975 & -0.75479 & 3.81849 \\
\hline $\mathrm{H}$ & -4.00265 & 0.84963 & 3.08885 \\
\hline $\mathrm{H}$ & -6.46302 & -0.94922 & 3.40248 \\
\hline $\mathrm{H}$ & -6.1394 & 0.52274 & 4.30962 \\
\hline $\mathrm{H}$ & -6.25633 & 1.87671 & 2.21834 \\
\hline $\mathrm{H}$ & -7.74426 & 0.94658 & 2.41011 \\
\hline $\mathrm{H}$ & -6.95482 & -0.75271 & 0.82048 \\
\hline $\mathrm{H}$ & -4.50891 & 1.04037 & 0.47933 \\
\hline $\mathrm{H}$ & -6.44781 & 1.56075 & -0.82383 \\
\hline $\mathrm{H}$ & -8.04542 & 2.42703 & -2.12659 \\
\hline $\mathrm{H}$ & -9.47284 & 2.82309 & -1.13862 \\
\hline $\mathrm{H}$ & -9.51845 & 1.42386 & -2.20585 \\
\hline $\mathrm{H}$ & 0.75289 & 3.31705 & 1.47066 \\
\hline $\mathrm{H}$ & -0.96452 & 2.94931 & 1.69878 \\
\hline $\mathrm{H}$ & -0.28913 & 3.43835 & 3.9897 \\
\hline $\mathrm{H}$ & 1.29159 & 2.6596 & 3.74481 \\
\hline $\mathrm{H}$ & 0.01996 & 0.88066 & 4.84831 \\
\hline $\mathrm{H}$ & -1.42802 & 1.25645 & 3.90546 \\
\hline $\mathrm{H}$ & -0.62948 & -0.43525 & $5 \quad 2.47094$ \\
\hline $\mathrm{H}$ & 0.48419 & -2.15296 & 3.02934 \\
\hline $\mathrm{H}$ & 1.47399 & -4.34073 & 2.7172 \\
\hline $\mathrm{H}$ & 1.1554 & -4.10972 & 4.45549 \\
\hline $\mathrm{H}$ & 2.72375 & -4.73649 & 3.90862 \\
\hline $\mathrm{H}$ & 2.31934 & -2.29254 & 5.73563 \\
\hline $\mathrm{H}$ & 3.92426 & -2.85146 & 5.19175 \\
\hline $\mathrm{H}$ & 3.43626 & -1.16951 & 4.92208 \\
\hline $\mathrm{H}$ & 2.39535 & -1.77181 & 1.09919 \\
\hline $\mathrm{H}$ & 5.22009 & -2.5359 & 0.66819 \\
\hline $\mathrm{H}$ & 4.46613 & 1.69899 & 1.50793 \\
\hline $\mathrm{H}$ & 6.23317 & 1.53553 & 1.74171 \\
\hline $\mathrm{H}$ & 5.56053 & 1.79001 & 0.09555 \\
\hline $\mathrm{H}$ & 3.6004 & -3.83535 & -0.78773 \\
\hline
\end{tabular}




\begin{tabular}{|c|c|c|}
\hline $\mathrm{H}$ & $3.07701-2.3125$ & -1.51463 \\
\hline $\mathrm{H}$ & $\begin{array}{lll}5.91682 & -4.69018\end{array}$ & -1.16601 \\
\hline $\mathrm{H}$ & $7.80904-4.83866$ & -2.75008 \\
\hline $\mathrm{H}$ & $8.05467-3.15308$ & -4.56283 \\
\hline $\mathrm{H}$ & $6.39044-1.31723$ & -4.76819 \\
\hline $\mathrm{H}$ & $\begin{array}{lll}4.50728 & -1.16278\end{array}$ & -3.16665 \\
\hline
\end{tabular}
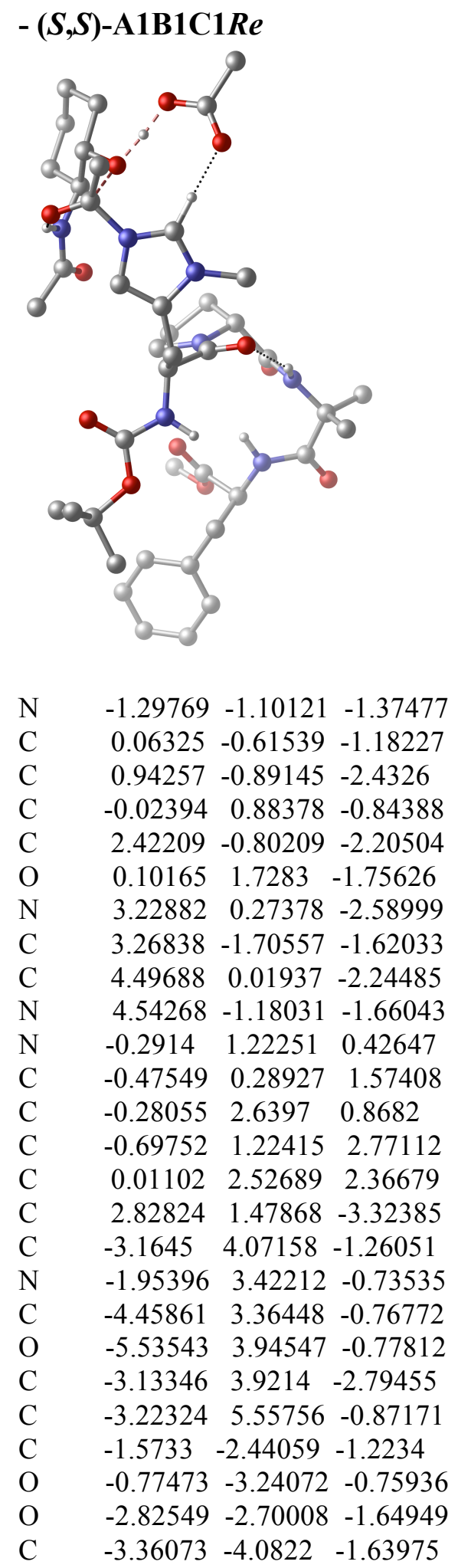

\begin{tabular}{|c|c|c|c|}
\hline & 3.4302 & & \\
\hline & -4.76118 & -3.90197 & -2.22752 \\
\hline & -2.50239 & -4.97384 & .5421 \\
\hline & -7.65422 & -2.56654 & 1.4239 \\
\hline & -6.61146 & -1.82388 & 0.8674 \\
\hline & .97501 & -2.29857 & 1.0623 \\
\hline & -6.87041 & -0.80341 & -0.0592 \\
\hline & -9.24563 & -1.2842 & 0.1420 \\
\hline & -5.74137 & 0.01965 & -0.6422 \\
\hline & -8.20143 & -0.54428 & -0.4126 \\
\hline & -5.40764 & 1.29341 & 0.1766 \\
\hline & -4.32598 & 2.03998 & -0.4467 \\
\hline & -4.96525 & 0.90935 & 1.5878 \\
\hline & -3.91428 & 0.34115 & 1.8235 \\
\hline & -5.86376 & 1.2512 & $2.5159^{\prime}$ \\
\hline & -5.52666 & 0.90582 & 3.8738 \\
\hline & -2.3632 & 3.74761 & 1.49052 \\
\hline & -1.64035 & 3.33769 & 0.58882 \\
\hline & 5.76264 & -1.84416 & $-1.0577^{\prime}$ \\
\hline & 7.00426 & -1.67241 & $-1.9055^{\prime}$ \\
\hline & 5.53831 & -2.86824 & -0.4193 \\
\hline & 6.2546 & -0.01127 & 4.43161 \\
\hline & 7.56827 & -0.03217 & 3.64377 \\
\hline & 5.26307 & -1.01179 & 3.83221 \\
\hline & 7.31759 & 0.25648 & 2.15903 \\
\hline & 4.99692 & -0.76541 & 2.33076 \\
\hline & 6.30883 & -0.71323 & 1.51282 \\
\hline & 6.05666 & -0.33924 & 0.16228 \\
\hline & 4.1341 & -1.82682 & 1.81172 \\
\hline & 2.81879 & -1.93188 & 2.10167 \\
\hline & 2.1979 & -1.08474 & 2.75596 \\
\hline & 7.5388 & 2.03896 & -1.64661 \\
\hline & 8.58599 & 3.13229 & -1.78311 \\
\hline & 6.67032 & 1.8953 & -2.52659 \\
\hline & 7.66176 & 1.32213 & -0.57726 \\
\hline & 2.13884 & -3.20021 & 1.5992 \\
\hline & -5.36991 & -3.24695 & -1.5987 \\
\hline & -5.26112 & -4.87244 & -2.2959 \\
\hline & -4.70695 & -3.47139 & -3.2315 \\
\hline & -4.01964 & -3.92129 & 0.4199 \\
\hline & -3.92168 & -5.57956 & -0.1921 \\
\hline & -2.43375 & -4.70345 & 0.2282 \\
\hline & -2.96908 & -5.96005 & -2.6275 \\
\hline & -1.49783 & -5.09685 & -2.1370 \\
\hline & -2.43361 & -4.54554 & -3.5472 \\
\hline & -1.92946 & -0.56386 & -1.9524 \\
\hline & 0.47405 & -1.16848 & -0.3374 \\
\hline & 0.71397 & -1.91354 & -2.7456 \\
\hline & 0.63368 & -0.21507 & -3.2332 \\
\hline & 3.07836 & -2.67451 & -1.1908 \\
\hline & 1.95567 & 1.92302 & -2.8455 \\
\hline & 3.67112 & 2.17008 & -3.3128 \\
\hline & 2.5892 & 1.2195 & -4.35897 \\
\hline & 5.34124 & 0.71145 & -2.3746 \\
\hline
\end{tabular}




\begin{tabular}{|c|c|c|c|}
\hline $\mathrm{H}$ & 6.95103 & -2.39703 & -2.72627 \\
\hline $\mathrm{H}$ & 7.12287 & -0.67107 & -2.3172 \\
\hline $\mathrm{H}$ & 7.87012 & -1.9147 & -1.28888 \\
\hline $\mathrm{H}$ & 9.58739 & 2.70008 & -1.70052 \\
\hline $\mathrm{H}$ & 8.47839 & 3.64957 & -2.73679 \\
\hline $\mathrm{H}$ & 8.47723 & 3.8471 & -0.96125 \\
\hline $\mathrm{H}$ & 6.84195 & 0.50454 & -0.27047 \\
\hline $\mathrm{H}$ & 8.25186 & 0.22397 & 1.59214 \\
\hline $\mathrm{H}$ & 6.93131 & 1.27744 & 2.04638 \\
\hline $\mathrm{H}$ & 8.04322 & -1.01722 & 3.75655 \\
\hline $\mathrm{H}$ & 8.27661 & 0.70223 & 4.04528 \\
\hline $\mathrm{H}$ & 6.42957 & -0.24406 & 5.48869 \\
\hline $\mathrm{H}$ & 5.82631 & 1.00048 & 4.4007 \\
\hline $\mathrm{H}$ & 4.30267 & -0.98364 & 4.35493 \\
\hline $\mathrm{H}$ & 5.66133 & -2.02976 & 3.94559 \\
\hline $\mathrm{H}$ & 4.56264 & -2.49596 & 1.17732 \\
\hline $\mathrm{H}$ & 1.25835 & -2.96198 & 0.99567 \\
\hline $\mathrm{H}$ & 1.78196 & -3.75945 & 2.46952 \\
\hline $\mathrm{H}$ & 2.80501 & -3.8486 & 1.02335 \\
\hline $\mathrm{H}$ & -1.33388 & -0.35983 & 1.38981 \\
\hline $\mathrm{H}$ & 0.42874 & -0.30711 & 1.72494 \\
\hline $\mathrm{H}$ & -0.28735 & 0.78625 & 3.68354 \\
\hline $\mathrm{H}$ & -1.76476 & 1.40824 & 2.91319 \\
\hline $\mathrm{H}$ & -0.36373 & 3.39754 & 2.90598 \\
\hline $\mathrm{H}$ & 1.09129 & 2.44116 & 2.52025 \\
\hline $\mathrm{H}$ & 0.4985 & 3.16616 & 0.30743 \\
\hline $\mathrm{H}$ & -1.25761 & 3.06927 & -1.38985 \\
\hline $\mathrm{H}$ & -3.07224 & 2.86854 & -3.0885 \\
\hline $\mathrm{H}$ & -2.27104 & 4.45171 & -3.21143 \\
\hline $\mathrm{H}$ & -4.04319 & 4.34741 & -3.2226 \\
\hline $\mathrm{H}$ & -2.37013 & 6.08487 & -1.30945 \\
\hline $\mathrm{H}$ & -4.15082 & 5.99636 & -1.24182 \\
\hline $\mathrm{H}$ & -3.19404 & 5.66775 & 0.21266 \\
\hline $\mathrm{H}$ & -3.39243 & 1.68224 & -0.29912 \\
\hline $\mathrm{H}$ & -6.27284 & 1.95573 & 0.22469 \\
\hline $\mathrm{H}$ & -4.61288 & 1.41988 & 4.17918 \\
\hline $\mathrm{H}$ & -6.37234 & 1.23518 & 4.47574 \\
\hline $\mathrm{H}$ & -5.38288 & -0.17281 & 3.96689 \\
\hline $\mathrm{H}$ & -5.99516 & 0.35209 & -1.65381 \\
\hline $\mathrm{H}$ & -4.83105 & -0.58646 & -0.71479 \\
\hline $\mathrm{H}$ & -8.41953 & 0.24226 & -1.13091 \\
\hline $\mathrm{H}$ & -10.2707 & -1.07037 & -0.14667 \\
\hline $\mathrm{H}$ & -9.78749 & -2.87728 & 1.49176 \\
\hline $\mathrm{H}$ & -7.43366 & -3.35587 & 2.13701 \\
\hline $\mathrm{H}$ & -5.58441 & -2.02967 & 1.15843 \\
\hline $\mathrm{H}$ & 6.74099 & -1.72991 & 1.52431 \\
\hline $\mathrm{H}$ & 4.47798 & 0.19359 & 2.20974 \\
\hline
\end{tabular}

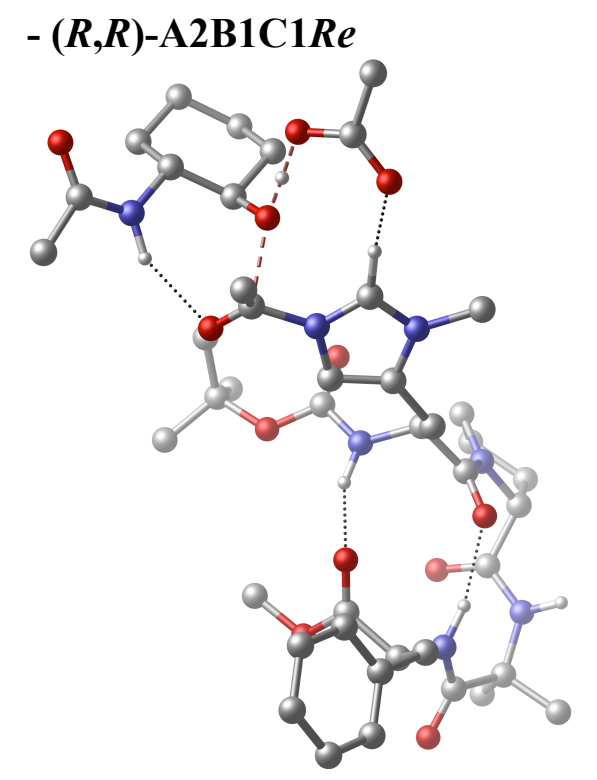

$\begin{array}{llll}\mathrm{N} & -0.98993 & -1.06936 & -0.26683 \\ \mathrm{C} & -1.13779 & -1.32468 & 1.15358 \\ \mathrm{C} & -0.7255 & -0.08561 & 1.98659 \\ \mathrm{C} & -2.61205 & -1.66407 & 1.46345 \\ \mathrm{C} & 0.71018 & 0.30398 & 1.81684 \\ \mathrm{O} & -3.40051 & -0.77645 & 1.82382 \\ \mathrm{~N} & 1.65952 & 0.23193 & 2.84468 \\ \mathrm{C} & 1.3851 & 0.79102 & 0.73132 \\ \mathrm{C} & 2.84526 & 0.66265 & 2.39292 \\ \mathrm{~N} & 2.69487 & 1.00849 & 1.11309 \\ \mathrm{~N} & -3.01924 & -2.94243 & 1.31114 \\ \mathrm{C} & -2.24245 & -4.10268 & 0.82768 \\ \mathrm{C} & -4.44182 & -3.26162 & 1.50817 \\ \mathrm{C} & -3.32981 & -5.02909 & 0.27505 \\ \mathrm{C} & -4.51359 & -4.77979 & 1.22289 \\ \mathrm{C} & 1.45726 & -0.23552 & 4.21512 \\ \mathrm{C} & -7.35407 & -0.98817 & 0.37644 \\ \mathrm{~N} & -6.36327 & -1.81104 & 1.08659 \\ \mathrm{C} & -6.74855 & 0.30556 & -0.24098 \\ \mathrm{O} & -7.42166 & 0.96625 & -1.02422 \\ \mathrm{C} & -8.38743 & -0.52551 & 1.42247 \\ \mathrm{C} & -8.05138 & -1.79383 & -0.73246 \\ \mathrm{C} & -0.12772 & -1.78181 & -1.05432 \\ \mathrm{O} & 0.47886 & -2.77914 & -0.68493 \\ \mathrm{O} & -0.07301 & -1.21568 & -2.28003 \\ \mathrm{C} & 0.81703 & -1.75529 & -3.332 \\ \mathrm{C} & 0.3816 & -3.17601 & -3.70065 \\ \mathrm{C} & 0.57461 & -0.79065 & -4.49467 \\ \mathrm{C} & 2.27395 & -1.68739 & -2.86626 \\ \mathrm{C} & -2.26138 & 5.7702 & -0.19019 \\ \mathrm{C} & -2.6865 & 4.52281 & 0.26983 \\ \mathrm{C} & -3.19635 & 6.74684 & -0.53435 \\ \mathrm{C} & -4.05118 & 4.23182 & 0.39549 \\ \mathrm{C} & -4.55936 & 6.46875 & -0.41496 \\ \mathrm{C} & -4.52566 & 2.87392 & 0.87201 \\ & & & \\ \mathrm{n} & & & \end{array}$




\begin{tabular}{|c|c|c|}
\hline $\mathrm{C}$ & $-4.98066 \quad 5.22201$ & 0.0462 \\
\hline $\mathrm{C}$ & $-4.90642 \quad 1.92639$ & -0.28334 \\
\hline $\mathrm{N}$ & $\begin{array}{ll}-5.52063 & 0.69732\end{array}$ & 0.20683 \\
\hline $\mathrm{C}$ & $-3.68754 \quad 1.53955$ & -1.1241 \\
\hline $\mathrm{O}$ & $\begin{array}{lll}-2.576 & 1.3214\end{array}$ & -0.6639 \\
\hline $\mathrm{O}$ & $-3.99108 \quad 1.4225$ & -2.41588 \\
\hline $\mathrm{C}$ & $-2.93018 \quad 0.98503$ & -3.28982 \\
\hline $\mathrm{O}$ & $-5.0614 \quad-2.45848$ & -0.68445 \\
\hline $\mathrm{C}$ & $\begin{array}{ll}-5.30901 & -2.45954\end{array}$ & 0.5157 \\
\hline $\mathrm{C}$ & $3.76945 \quad 1.52188$ & 0.1919 \\
\hline $\mathrm{C}$ & $4.55894 \quad 2.66416$ & 0.78815 \\
\hline $\mathrm{O}$ & $3.52797 \quad 1.39208$ & -1.00124 \\
\hline $\mathrm{C}$ & $7.4197 \quad-2.79206$ & -1.32189 \\
\hline $\mathrm{C}$ & $6.18189-3.2606$ & -0.54598 \\
\hline $\mathrm{C}$ & $7.15382-1.45134$ & -2.01622 \\
\hline $\mathrm{C}$ & $5.72011-2.18843$ & 0.45036 \\
\hline $\mathrm{C}$ & $6.7017-0.36335$ & -1.02024 \\
\hline $\mathrm{C}$ & $5.46683-0.82515$ & -0.21734 \\
\hline $\mathrm{O}$ & $5.06569 \quad 0.11399$ & 0.76396 \\
\hline $\mathrm{N}$ & $6.39713 \quad 0.88961$ & -1.70945 \\
\hline $\mathrm{C}$ & $\begin{array}{lll}7.36381 & 1.695 & -\end{array}$ & -2.22729 \\
\hline $\mathrm{O}$ & $8.56308 \quad 1.4337$ & -2.16982 \\
\hline $\mathrm{C}$ & $6.3661 \quad 0.59408$ & 3.67835 \\
\hline $\mathrm{C}$ & $7.48193 \quad 0.58307$ & 4.71305 \\
\hline $\mathrm{O}$ & $5.18624 \quad 0.78442$ & 4.04695 \\
\hline $\mathrm{O}$ & $6.75636 \quad 0.40551$ & 2.46656 \\
\hline $\mathrm{C}$ & $6.85615 \quad 2.96458$ & -2.90368 \\
\hline $\mathrm{H}$ & $-0.47429-0.81128$ & -4.80574 \\
\hline $\mathrm{H}$ & $1.19176-1.07733$ & -5.35084 \\
\hline $\mathrm{H}$ & $0.83624 \quad 0.23195$ & -4.20867 \\
\hline $\mathrm{H}$ & $-0.67573 \quad-3.19089$ & -3.98377 \\
\hline $\mathrm{H}$ & $0.96698 \quad-3.52612$ & -4.55676 \\
\hline $\mathrm{H}$ & $0.53702-3.86113$ & -2.86706 \\
\hline $\mathrm{H}$ & $2.93384-1.94165$ & -3.70197 \\
\hline $\mathrm{H}$ & $2.45726-2.38768$ & -2.05076 \\
\hline $\mathrm{H}$ & $2.52459-0.67757$ & -2.52765 \\
\hline $\mathrm{H}$ & $-1.45486-0.25138$ & -0.6541 \\
\hline $\mathrm{H}$ & $\begin{array}{lll}-0.48802 & -2.172\end{array}$ & 1.38443 \\
\hline $\mathrm{H}$ & $\begin{array}{ll}-1.37938 & 0.73787\end{array}$ & 1.68875 \\
\hline $\mathrm{H}$ & $-0.94304-0.27825$ & 3.04087 \\
\hline $\mathrm{H}$ & $1.05792 \quad 0.99647$ & -0.27352 \\
\hline $\mathrm{H}$ & $1.12077-1.27471$ & 4.21646 \\
\hline $\mathrm{H}$ & $2.41235-0.16496$ & 4.73651 \\
\hline $\mathrm{H}$ & $0.71931 \quad 0.38786$ & 4.72524 \\
\hline $\mathrm{H}$ & $3.78239 \quad 0.69348$ & 2.97641 \\
\hline $\mathrm{H}$ & $3.95277 \quad 3.57452$ & 0.7066 \\
\hline $\mathrm{H}$ & $4.81983 \quad 2.4974$ & 1.83326 \\
\hline $\mathrm{H}$ & $5.4714 \quad 2.79008$ & 0.20661 \\
\hline $\mathrm{H}$ & $8.24447 \quad 1.31862$ & 4.44067 \\
\hline $\mathrm{H}$ & $7.09051 \quad 0.80131$ & 5.70731 \\
\hline $\mathrm{H}$ & $7.97034-0.39651$ & 4.71429 \\
\hline $\mathrm{H}$ & $5.86764 \quad 0.29162$ & 1.59141 \\
\hline $\mathrm{H}$ & $6.48325-2.04861$ & 1.22653 \\
\hline $\mathrm{H}$ & $4.80089-2.496$ & 0.96175 \\
\hline
\end{tabular}

$\begin{array}{llll}\mathrm{H} & 6.38762 & -4.19851 & -0.0159 \\ \mathrm{H} & 5.37011 & -3.47596 & -1.25578 \\ \mathrm{H} & 8.26473 & -2.68315 & -0.62802 \\ \mathrm{H} & 7.71851 & -3.54541 & -2.06056 \\ \mathrm{H} & 6.36753 & -1.5783 & -2.77449 \\ \mathrm{H} & 8.04627 & -1.08901 & -2.53331 \\ \mathrm{H} & 7.52272 & -0.16768 & -0.31858 \\ \mathrm{H} & 4.62812 & -0.92697 & -0.92545 \\ \mathrm{H} & 5.4233 & 1.16419 & -1.77717 \\ \mathrm{H} & 5.77737 & 3.11389 & -2.80388 \\ \mathrm{H} & 7.11308 & 2.92642 & -3.96649 \\ \mathrm{H} & 7.3814 & 3.82221 & -2.47499 \\ \mathrm{H} & -1.50006 & -3.80987 & 0.08594 \\ \mathrm{H} & -1.71499 & -4.57437 & 1.66868 \\ \mathrm{H} & -3.01146 & -6.07433 & 0.25171 \\ \mathrm{H} & -3.59833 & -4.71516 & -0.73652 \\ \mathrm{H} & -5.47568 & -5.07048 & 0.79461 \\ \mathrm{H} & -4.38185 & -5.33251 & 2.15955 \\ \mathrm{H} & -4.72951 & -3.02458 & 2.53847 \\ \mathrm{H} & -6.37963 & -1.76818 & 2.09523 \\ \mathrm{H} & -7.90976 & 0.04251 & 2.22931 \\ \mathrm{H} & -8.90394 & -1.3881 & 1.85545 \\ \mathrm{H} & -9.12311 & 0.12151 & 0.94228 \\ \mathrm{H} & -8.58484 & -2.64118 & -0.29089 \\ \mathrm{H} & -8.76074 & -1.14716 & -1.24995 \\ \mathrm{H} & -7.32313 & -2.16731 & -1.45237 \\ \mathrm{H} & -4.9644 & 0.09673 & 0.80968 \\ \mathrm{H} & -5.64379 & 2.39231 & -0.93936 \\ \mathrm{H} & -2.59392 & -0.01681 & -3.01562 \\ \mathrm{H} & -3.36358 & 0.9838 & -4.28869 \\ \mathrm{H} & -2.08586 & 1.67545 & -3.23503 \\ \mathrm{H} & -5.41941 & 2.98614 & 1.49448 \\ \mathrm{H} & -3.75898 & 2.38835 & 1.48333 \\ \mathrm{H} & -6.0439 & 5.01414 & 0.1414 \\ \mathrm{H} & -5.29472 & 7.22393 & -0.67724 \\ \mathrm{H} & -2.8663 & 7.71833 & -0.8904 \\ \mathrm{H} & -1.1989 & 5.97912 & -0.27768 \\ \mathrm{H} & -1.95603 & 3.76197 & 0.52825 \\ & & & \\ & & & \end{array}$




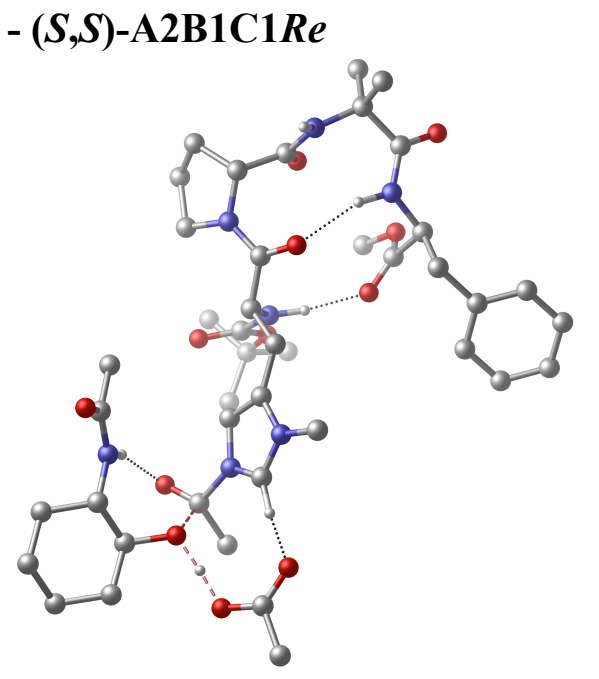

$\begin{array}{llll}\mathrm{N} & 0.96216 & -0.80469 & 1.10243 \\ \mathrm{C} & 0.611 & -1.06701 & -0.28499 \\ \mathrm{C} & 0.016 & 0.17162 & -0.97799 \\ \mathrm{C} & 1.87082 & -1.51293 & -1.06106 \\ \mathrm{C} & -1.33943 & 0.55331 & -0.4716 \\ \mathrm{O} & 2.5227 & -0.70233 & -1.73516 \\ \mathrm{~N} & -2.01178 & 1.67842 & -0.95857 \\ \mathrm{C} & -2.18496 & -0.0167 & 0.43849 \\ \mathrm{C} & -3.2098 & 1.77417 & -0.36531 \\ \mathrm{~N} & -3.32765 & 0.75978 & 0.4912 \\ \mathrm{~N} & 2.23996 & -2.8098 & -0.97421 \\ \mathrm{C} & 1.61952 & -3.89108 & -0.18415 \\ \mathrm{C} & 3.45099 & -3.25343 & -1.67793 \\ \mathrm{C} & 2.77556 & -4.87975 & -0.00709 \\ \mathrm{C} & 3.53876 & -4.76013 & -1.33548 \\ \mathrm{C} & -1.53994 & 2.61149 & -1.97977 \\ \mathrm{C} & 6.73978 & -1.25461 & -1.96022 \\ \mathrm{~N} & 5.48721 & -2.00057 & -2.16006 \\ \mathrm{C} & 6.52093 & 0.09195 & -1.21251 \\ \mathrm{O} & 7.47941 & 0.67056 & -0.71644 \\ \mathrm{C} & 7.28974 & -0.9033 & -3.35561 \\ \mathrm{C} & 7.76129 & -2.09904 & -1.18086 \\ \mathrm{C} & 0.3499 & -1.42951 & 2.14699 \\ \mathrm{O} & -0.50986 & -2.29932 & 2.02361 \\ \mathrm{O} & 0.83606 & -0.95452 & 3.31307 \\ \mathrm{C} & 0.29983 & -1.41792 & 4.6103 \\ \mathrm{C} & 0.56069 & -2.91752 & 4.77963 \\ \mathrm{C} & 1.12294 & -0.60541 & 5.61232 \\ \mathrm{C} & -1.18694 & -1.0667 & 4.71732 \\ \mathrm{C} & 2.77533 & 5.69808 & 0.88319 \\ \mathrm{C} & 2.88585 & 4.46591 & 0.23697 \\ \mathrm{C} & 3.77735 & 6.65854 & 0.7401 \\ \mathrm{C} & 3.99708 & 4.1736 & -0.56509 \\ \mathrm{C} & 4.89119 & 6.37838 & -0.05322 \\ \mathrm{C} & 4.13917 & 2.83557 & -1.26349 \\ \mathrm{C} & 4.99738 & 5.14689 & -0.6992 \\ \mathrm{C} & 4.93459 & 1.80151 & -0.44797\end{array}$

\begin{tabular}{|c|c|c|c|}
\hline & 5.2587 & 0.61593 & -1.23892 \\
\hline $\mathrm{C}$ & 4.16662 & 1.29094 & 0.77469 \\
\hline $\mathrm{O}$ & 2.94754 & 1.25218 & 0.85514 \\
\hline $\mathrm{O}$ & 4.99483 & 0.80585 & 1.69752 \\
\hline $\mathrm{C}$ & 4.40439 & 0.03547 & 2.76401 \\
\hline $\mathrm{O}$ & 4.91272 & -2.35416 & 0.02857 \\
\hline $\mathrm{C}$ & 4.68379 & -2.48046 & -1.17037 \\
\hline C & -4.52533 & 0.41493 & 1.35382 \\
\hline $\mathrm{C}$ & -4.99299 & 1.58271 & 2.19527 \\
\hline $\mathrm{O}$ & -4.55623 & -0.74733 & 31.74465 \\
\hline $\mathrm{C}$ & -8.73177 & -2.03439 & -1.71292 \\
\hline C & -9.16215 & -1.02208 & -0.64457 \\
\hline $\mathrm{C}$ & -7.33565 & -2.58543 & -1.40329 \\
\hline $\mathrm{C}$ & -8.13025 & 0.1045 & -0.50876 \\
\hline $\mathrm{C}$ & -6.28249 & -1.46773 & -1.25136 \\
\hline C & -6.71894 & -0.42556 & -0.20036 \\
\hline $\mathrm{O}$ & -5.77816 & 0.63897 & -0.11877 \\
\hline N & -4.98692 & -2.03652 & -0.88246 \\
\hline$C$ & -4.20463 & -2.69303 & -1.77564 \\
\hline $\mathrm{O}$ & -4.49121 & -2.81973 & -2.96571 \\
\hline $\mathrm{C}$ & -6.21358 & 3.81188 & -0.91245 \\
\hline C & -7.12588 & 4.98304 & -1.24217 \\
\hline $\mathrm{O}$ & -5.00148 & 3.86998 & -1.18944 \\
\hline $\mathrm{O}$ & -6.81251 & 2.81697 & -0.34235 \\
\hline C & -2.92708 & -3.3065 & -1.20832 \\
\hline $\mathrm{H}$ & 2.18912 & -0.82733 & 5.50883 \\
\hline H & 0.81782 & -0.85076 & 6.63352 \\
\hline $\mathrm{H}$ & 0.97479 & 0.46669 & 5.455 \\
\hline $\mathrm{H}$ & 1.62439 & -3.13693 & 4.64282 \\
\hline $\mathrm{H}$ & 0.28007 & -3.22535 & 5.79193 \\
\hline $\mathrm{H}$ & -0.01811 & -3.49994 & 4.0629 \\
\hline H & -1.54546 & -1.3046 & 5.72372 \\
\hline H & -1.78305 & -1.62524 & 3.99542 \\
\hline $\mathrm{H}$ & -1.3368 & 0.00449 & 4.55021 \\
\hline $\mathrm{H}$ & 1.64341 & -0.07143 & 1.2912 \\
\hline $\mathrm{H}$ & -0.12355 & -1.87566 & -0.27368 \\
\hline $\mathrm{H}$ & 0.72553 & 0.99819 & -0.86133 \\
\hline$H_{1}$ & -0.03587 & -0.02741 & -2.05578 \\
\hline $\mathrm{H}$ & -2.07433 & -0.89341 & 1.05634 \\
\hline $\mathrm{H}$ & -1.35256 & 2.0809 & -2.91625 \\
\hline $\mathrm{H}$ & -2.31576 & 3.36112 & -2.13761 \\
\hline त & -0.61944 & 3.09913 & -1.64991 \\
\hline $\mathrm{H}$ & -3.95976 & 2.54296 & -0.60596 \\
\hline त & -4.27845 & 1.72218 & 3.01496 \\
\hline त & -5.08436 & 2.51088 & 1.63232 \\
\hline त & -5.96346 & 1.32695 & 2.62188 \\
\hline H & -7.69138 & 5.27731 & -0.35341 \\
\hline H & -6.54743 & 5.82799 & -1.61705 \\
\hline & -7.85513 & 4.67396 & -1.998 \\
\hline & -6.25286 & 1.73937 & -0.23719 \\
\hline & -8.42317 & 0.8121 & 0.27294 \\
\hline & -8.09017 & 0.68152 & -1.44106 \\
\hline & -9.27362 & -1.5387 & 0.3195 \\
\hline & -10.1439 & & 10884 \\
\hline
\end{tabular}




\begin{tabular}{|c|c|c|c|}
\hline $\mathrm{H}$ & -9.45379 & -2.857 & -1.78026 \\
\hline $\mathrm{H}$ & -8.72441 & -1.54475 & -2.69684 \\
\hline $\mathrm{H}$ & -6.99615 & -3.26773 & -2.18776 \\
\hline${ }_{H}^{H}$ & -7.3677 & -3.15905 & -0.46619 \\
\hline H & -4.68316 & -1.91426 & $5 \quad 0.07905$ \\
\hline${ }_{H}^{H}$ & -2.73836 & -3.06658 & -0.15897 \\
\hline H & -2.0817 & -2.97603 & -1.81916 \\
\hline $\mathrm{H}$ & -2.99218 & -4.39388 & -1.31292 \\
\hline $\mathrm{H}$ & 1.21563 & -3.52 & 0.75643 \\
\hline $\mathrm{H}$ & 0.79459 & -4.34524 & -0.75017 \\
\hline $\mathrm{H}$ & 2.42682 & -5.89507 & 0.19713 \\
\hline $\mathrm{H}$ & 3.41611 & -4.55185 & 0.81581 \\
\hline $\mathrm{H}$ & 4.57288 & -5.10631 & -1.26949 \\
\hline $\mathrm{H}$ & 3.04023 & -5.34006 & -2.11947 \\
\hline $\mathrm{H}$ & 3.32631 & -3.08752 & -2.75404 \\
\hline $\mathrm{H}$ & 5.13968 & -2.06487 & -3.1058 \\
\hline $\mathrm{H}$ & 6.56815 & -0.31172 & -3.93005 \\
\hline $\mathrm{H}$ & 7.52707 & -1.81454 & -3.91445 \\
\hline $\mathrm{H}$ & 8.20149 & -0.31361 & -3.24576 \\
\hline $\mathrm{H}$ & 8.01408 & -2.99457 & -1.75678 \\
\hline $\mathrm{H}$ & 8.66243 & -1.50903 & -1.01083 \\
\hline $\mathrm{H}$ & 7.35148 & -2.39792 & -0.21552 \\
\hline $\mathrm{H}$ & 4.47726 & 0.05026 & -1.55969 \\
\hline $\mathrm{H}$ & 5.88953 & 2.21404 & -0.11573 \\
\hline $\mathrm{H}$ & 4.0633 & -0.92312 & 2.36641 \\
\hline $\mathrm{H}$ & 5.20495 & -0.11873 & 3.48603 \\
\hline $\mathrm{H}$ & 3.56925 & 0.57263 & 3.21647 \\
\hline $\mathrm{H}$ & 4.67267 & 2.96714 & -2.21019 \\
\hline $\mathrm{H}$ & 3.15715 & 2.41386 & -1.49843 \\
\hline $\mathrm{H}$ & 5.86501 & 4.93856 & -1.32086 \\
\hline $\mathrm{H}$ & 5.67548 & 7.12022 & -0.17345 \\
\hline $\mathrm{H}$ & 3.6905 & 7.61835 & 1.24085 \\
\hline $\mathrm{H}$ & 1.90475 & 5.90768 & 1.49839 \\
\hline $\mathrm{H}$ & 2.11233 & 3.71473 & 0.36384 \\
\hline $\mathrm{H}$ & -6.74032 & -0.93715 & 0.77745 \\
\hline E & & -0.95927 & \\
\hline
\end{tabular}

\begin{tabular}{|c|c|c|c|}
\hline & 2.41196 & -1.24625 & -1.69608 \\
\hline & 1.7472 & -0.56863 & -2.79304 \\
\hline & 0.70725 & -1.4918 & -3.50718 \\
\hline & 1.06428 & 0.69064 & -2.24946 \\
\hline & -0.55561 & -1.77053 & -2.75457 \\
\hline$U$ & 0.78366 & 0.78774 & -1.04977 \\
\hline $\mathbb{N}$ & -0.73428 & -2.79536 & -1.81722 \\
\hline 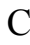 & -1.75926 & -1.12837 & -2.82898 \\
\hline C & -1.99059 & -2.76247 & -1.35577 \\
\hline N & -2.62966 & -1.76023 & -1.96638 \\
\hline N & 0.70457 & 1.6478 & -3.14098 \\
\hline & 1.17175 & 1.81476 & -4.53154 \\
\hline 0 & -0.04312 & 2.81789 & -2.6543 \\
\hline C & 0.94049 & 3.3065 & -4.80781 \\
\hline O & -0.28096 & 3.64117 & -3.93853 \\
\hline C & 0.25321 & -3.77932 & -1.36699 \\
\hline C & 0.64653 & 4.77816 & 0.6047 \\
\hline $\mathrm{N}$ & 0.10596 & 4.01859 & -0.5355 \\
\hline C & 1.62565 & 3.94373 & 1.48077 \\
\hline O & 2.24295 & 4.50197 & 2.38112 \\
\hline C & -0.54583 & 5.14273 & 1.51035 \\
\hline C & 1.35892 & 6.05908 & 0.1382 \\
\hline C & 3.37891 & -2.16855 & -2.00089 \\
\hline O & 3.59894 & -2.56946 & -3.13829 \\
\hline O & 3.98377 & -2.59743 & -0.87292 \\
\hline C & 5.13091 & -3.52334 & -0.93205 \\
\hline C & 6.27486 & -2.88154 & -1.72316 \\
\hline C & 5.5095 & -3.67735 & 0.54216 \\
\hline C & 4.69459 & -4.86743 & -1.52373 \\
\hline C & 4.05065 & -2.2286 & 3.9794 \\
\hline C & 3.29049 & -1.46996 & 3.08733 \\
\hline C & 4.12162 & -1.87034 & 5.32594 \\
\hline C & 2.58674 & -0.34156 & 3.52968 \\
\hline C & 3.42639 & -0.74704 & 5.7772 \\
\hline C & 1.77326 & 0.50365 & 2.57118 \\
\hline C & 2.66641 & 0.00852 & 4.88501 \\
\hline C & 2.54102 & 1.73641 & 2.05228 \\
\hline$N$ & 1.67938 & 2.60044 & 1.25378 \\
\hline C & 3.7409 & 1.32865 & 1.19192 \\
\hline $\mathrm{O}$ & 3.72785 & 0.45425 & 0.34343 \\
\hline $\mathrm{O}$ & 4.81333 & 2.08516 & 1.45137 \\
\hline C & 5.9707 & 1.84192 & 0.63135 \\
\hline $\mathrm{O}$ & 1.98644 & 3.83962 & -1.83962 \\
\hline C & 0.80211 & 3.60312 & -1.62955 \\
\hline C & -4.03972 & -1.30947 & -1.72716 \\
\hline$C$ & -5.03245 & -2.44364 & -1.65415 \\
\hline O & -4.28928 & -0.18192 & -2.12959 \\
\hline C & -4.0909 & 1.8458 & 3.22518 \\
\hline O & -2.69372 & 1.41144 & 2.76339 \\
\hline O & -5.07594 & 1.86034 & 2.04984 \\
\hline 0 & -2.74417 & 0.05513 & 2.04779 \\
\hline 0 & -5.14579 & 0.4925 & 1.3408 \\
\hline & -3.74502 & 0.03655 & 0.87824 \\
\hline & -3.75287 & -1.24084 & 0.27844 \\
\hline
\end{tabular}

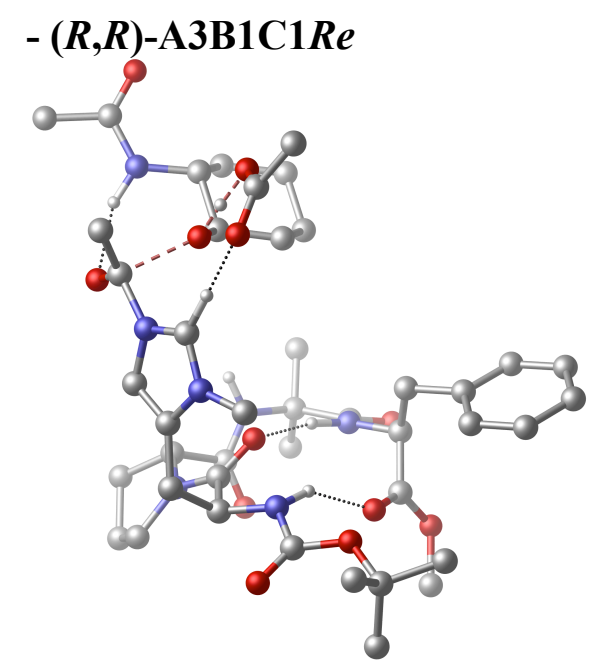




\begin{tabular}{|c|c|c|}
\hline & 0.53462 & 0.19378 \\
\hline$r^{\prime}$ & -7.40307 & 0.33252 \\
\hline $\mathrm{O}$ & 0.60796 & 1.42315 \\
\hline $\mathrm{C}$ & $-3.80481-4.16095$ & 1.63205 \\
\hline C & $\begin{array}{ll}-4.08927 & -5.18202\end{array}$ & 2.72416 \\
\hline U & $-3.12 \quad-4.49726$ & 0.64 \\
\hline $\mathrm{O}$ & $-4.3244 \quad-3.00161$ & 1.8323 \\
\hline $\mathrm{C}$ & $\begin{array}{ll}-8.19023 & 0.58524\end{array}$ & -0.97371 \\
\hline $\mathrm{H}$ & $\begin{array}{lll}5.75366 & -2.70621\end{array}$ & 0.98092 \\
\hline $\mathrm{H}$ & $6.37858-4.33457$ & 0.63918 \\
\hline H & $4.68171-4.11413$ & 1.10866 \\
\hline $\mathrm{H}$ & $6.52454-1.90407$ & -1.29913 \\
\hline$\pi$ & $7.1641-3.51681$ & -1.66266 \\
\hline H & $6.00582-2.75536$ & -2.77229 \\
\hline H & $\begin{array}{lll}5.51916 & -5.58393\end{array}$ & -1.45235 \\
\hline $\mathrm{H}$ & $4.40948-4.76146$ & -2.5703 \\
\hline H & $\begin{array}{lll}3.84809 & -5.27304\end{array}$ & -0.96019 \\
\hline H & $2.51238-0.72883$ & -0.82403 \\
\hline H & $2.48531-0.30575$ & -3.55999 \\
\hline H & $0.42836-1.02622$ & -4.45702 \\
\hline H & $1.23756-2.41365$ & -3.75933 \\
\hline H & $\begin{array}{ll}-2.08139 & -0.28462\end{array}$ & -3.41554 \\
\hline $\mathrm{H}$ & $1.06508-3.27559$ & -0.84345 \\
\hline H & $-0.25194-4.4766$ & -0.6984 \\
\hline $\mathrm{H}$ & $0.65452-4.32233$ & -2.22544 \\
\hline H & $-2.41733-3.4159$ & -0.57444 \\
\hline$H$ & $\begin{array}{lll}-5.25931 & -2.76087\end{array}$ & -2.67942 \\
\hline $\mathrm{H}$ & $-4.66158-3.29645$ & -1.08516 \\
\hline$H$ & $\begin{array}{ll}-5.9438 & -2.06917\end{array}$ & -1.18935 \\
\hline 1 & $-5.17039-5.2965$ & 2.84766 \\
\hline $\mathrm{H}$ & $\begin{array}{ll}-3.63877 & -6.14496\end{array}$ & 2.48101 \\
\hline H & $\begin{array}{lll}-3.69637 & -4.81801\end{array}$ & 3.67858 \\
\hline $\mathrm{H}$ & $-4.04489-2.10461$ & 0.99907 \\
\hline $\mathrm{H}$ & $\begin{array}{ll}-3.03789 & -0.72863\end{array}$ & 2.75727 \\
\hline $\mathrm{H}$ & $\begin{array}{ll}-1.7579 & -0.22299\end{array}$ & 1.65908 \\
\hline H & $-2.001 \quad 1.36373$ & 3.61259 \\
\hline $\mathrm{H}$ & $-2.29058 \quad 2.1703$ & 2.07531 \\
\hline $\mathrm{H}$ & $-4.45101 \quad 1.14864$ & 3.99392 \\
\hline $\mathrm{H}$ & $-4.05077 \quad 2.83506$ & 3.69662 \\
\hline H & $-4.765 \quad 2.61842$ & 1.31588 \\
\hline $\mathrm{H}$ & -6.083312 .12558 & 2.38128 \\
\hline$\theta$ & $-5.53794-0.24826$ & 2.04949 \\
\hline$\theta$ & $\begin{array}{ll}-3.40472 \quad 0.75181\end{array}$ & 0.10821 \\
\hline 1 & -5.63945 & -0.73216 \\
\hline 1 & -7.56324 & -1.86523 \\
\hline 1 & $\begin{array}{ll}-8.76551 & 1.51378\end{array}$ & -1.03314 \\
\hline 1 & $\begin{array}{lll}-8.90873 & -0.23873\end{array}$ & -0.95415 \\
\hline$\theta$ & $2.22266 \quad 1.5329$ & -4.62927 \\
\hline & 0.58296 & -5.2155 \\
\hline & $0.78174 \quad 3.50964$ & -5.86977 \\
\hline & $1.8041 \quad 3.87757$ & -4.46018 \\
\hline & $-0.37938 \quad 4.70897$ & -3.72941 \\
\hline & $-1.20639 \quad 3.30633$ & -4.41965 \\
\hline & -0.97999 & -2.19827 \\
\hline
\end{tabular}

$\begin{array}{llll}\mathrm{H} & -0.83254 & 3.6604 & -0.42789 \\ \mathrm{H} & -1.07758 & 4.24694 & 1.8509 \\ \mathrm{H} & -1.24802 & 5.78848 & 0.97379 \\ \mathrm{H} & -0.1791 & 5.67151 & 2.39106 \\ \mathrm{H} & 0.64575 & 6.71002 & -0.37692 \\ \mathrm{H} & 1.75713 & 6.57787 & 1.01115 \\ \mathrm{H} & 2.17822 & 5.82302 & -0.54038 \\ \mathrm{H} & 1.21393 & 2.18483 & 0.45327 \\ \mathrm{H} & 2.90445 & 2.34688 & 2.8804 \\ \mathrm{H} & 5.73776 & 2.02917 & -0.41934 \\ \mathrm{H} & 6.72993 & 2.53754 & 0.98606 \\ \mathrm{H} & 6.30923 & 0.80954 & 0.746 \\ \mathrm{H} & 0.873 & 0.8765 & 3.07063 \\ \mathrm{H} & 1.4456 & -0.09149 & 1.71307 \\ \mathrm{H} & 2.12159 & 0.87831 & 5.24453 \\ \mathrm{H} & 3.47114 & -0.46195 & 6.82438 \\ \mathrm{H} & 4.71054 & -2.4634 & 6.01957 \\ \mathrm{H} & 4.58584 & -3.10359 & 3.62136 \\ \mathrm{H} & 3.24634 & -1.75117 & 2.03898\end{array}$

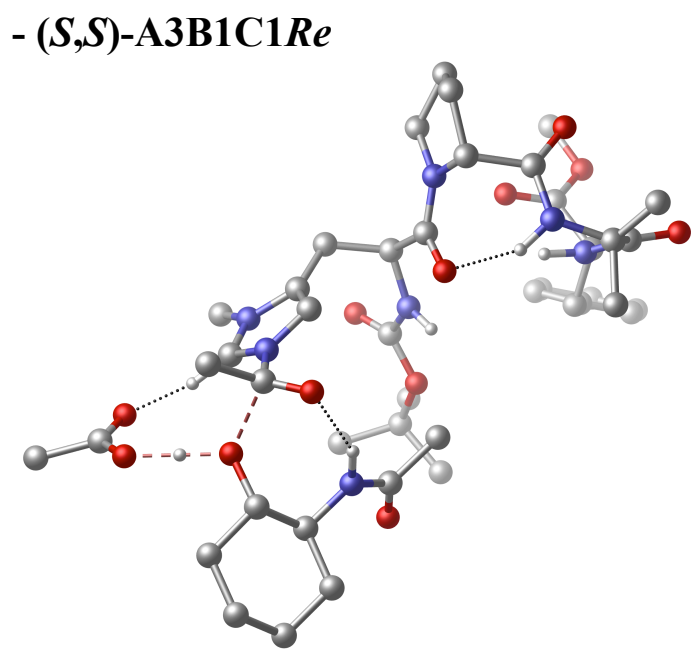

\begin{tabular}{|c|c|c|c|}
\hline & -0.65717 & 0.52968 & $3-0.79689$ \\
\hline & -0.93393 & -0.55361 & $1-1.71213$ \\
\hline & 0.24246 & -0.86084 & -2.68883 \\
\hline & -1.29869 & -1.77385 & $5-0.85436$ \\
\hline & 1.60053 & -0.9757 & -2.0653 \\
\hline & -0.86725 & -1.85673 & $\begin{array}{ll}3 & 0.31202\end{array}$ \\
\hline & 2.70255 & -0.28341 & -2.57474 \\
\hline & 2.08389 & -1.71584 & -1.0207 \\
\hline & 3.79726 & -0.60317 & -1.87342 \\
\hline & 3.44321 & -1.47662 & $2-0.92757$ \\
\hline & -2.06172 & -2.74033 & $3-1.40192$ \\
\hline & -2.69625 & -2.73147 & $7-2.74057$ \\
\hline & -2.38835 & -3.96912 & $2-0.63647$ \\
\hline & -3.57357 & -3.99355 & $5-2.7384$ \\
\hline & -2.89013 & -4.92581 & $1-1.7248$ \\
\hline & 2.71246 & 0.68891 & -3.6723 \\
\hline & -3.85945 & -2.80838 & 2.7420 \\
\hline & -3.0078 & -3.242 & 1.62037 \\
\hline
\end{tabular}




\begin{tabular}{|c|c|c|c|}
\hline $\mathrm{C}$ & -4.76156 & -1.60564 & 2.33607 \\
\hline $\mathrm{O}$ & -5.85569 & -1.42218 & 2.84989 \\
\hline $\mathrm{C}$ & -2.92113 & -2.32241 & 3.86449 \\
\hline C & -4.74041 & -3.9597 & 3.24641 \\
\hline C & -0.39094 & 1.80353 & -1.21327 \\
\hline $\mathrm{O}$ & -0.30256 & 2.12698 & -2.39459 \\
\hline $\mathrm{O}$ & -0.27214 & 2.60966 & -0.14175 \\
\hline $\mathrm{C}$ & 0.18918 & 4.0179 & -0.25565 \\
\hline $\mathrm{C}$ & -0.79646 & 4.81839 & -1.11038 \\
\hline $\mathrm{C}$ & 0.1726 & 4.49077 & 1.199 \\
\hline $\mathrm{C}$ & 1.61338 & 4.03963 & -0.81372 \\
\hline $\mathrm{C}$ & -5.24873 & 4.52295 & -1.26451 \\
\hline $\mathrm{C}$ & -4.57061 & 3.42974 & -0.72185 \\
\hline $\mathrm{C}$ & -6.28106 & 5.13153 & -0.54977 \\
\hline $\mathrm{C}$ & -4.91215 & 2.92901 & 0.54214 \\
\hline $\mathrm{C}$ & -6.62966 & 4.6415 & 0.71029 \\
\hline C & -4.1938 & 1.72808 & 1.11893 \\
\hline C & -5.95009 & 3.54972 & 1.24989 \\
\hline C & -4.93138 & 0.38193 & 0.8797 \\
\hline $\mathrm{N}$ & -4.19263 & -0.72837 & 1.4517 \\
\hline C & -5.10467 & 0.14163 & -0.61887 \\
\hline $\mathrm{O}$ & -4.19849 & -0.20938 & -1.35551 \\
\hline $\mathrm{O}$ & -6.35119 & 0.38878 & -1.02942 \\
\hline C & -6.59239 & 0.25939 & -2.44291 \\
\hline $\mathrm{O}$ & -4.6775 & -3.8378 & 0.17257 \\
\hline C & -3.48708 & -3.67913 & 0.42433 \\
\hline C & 4.33076 & -2.1769 & 0.08799 \\
\hline C & 5.33144 & -3.10758 & -0.56113 \\
\hline $\mathrm{O}$ & 3.79909 & -2.40538 & 1.16619 \\
\hline C & 6.96138 & 1.50807 & 3.69889 \\
\hline $\mathrm{C}$ & 7.82426 & 0.3727 & 3.13455 \\
\hline $\mathrm{C}$ & 5.47453 & 1.14206 & 3.63132 \\
\hline C & 7.39926 & 0.01967 & 1.70332 \\
\hline C & 5.02811 & 0.77134 & 2.20173 \\
\hline $\mathrm{C}$ & 5.9086 & -0.34724 & 1.61046 \\
\hline $\mathrm{O}$ & 5.53176 & -0.61999 & 0.265 \\
\hline $\mathrm{N}$ & 3.62819 & 0.35113 & 2.19498 \\
\hline $\mathrm{C}$ & 2.60235 & 1.22728 & 2.30629 \\
\hline $\mathrm{O}$ & 2.7629 & 2.4505 & 2.36729 \\
\hline $\mathrm{C}$ & 7.28834 & 0.08133 & -2.39665 \\
\hline $\mathrm{C}$ & 8.60732 & 0.28594 & -3.12715 \\
\hline $\mathrm{O}$ & 6.23754 & 0.5607 & -2.86231 \\
\hline $\mathrm{O}$ & 7.38007 & -0.60629 & -1.30465 \\
\hline $\mathrm{C}$ & 1.21756 & 0.60164 & 2.40687 \\
\hline $\mathrm{H}$ & -0.83356 & 4.40026 & 1.61985 \\
\hline $\mathrm{H}$ & 0.46653 & 5.54411 & 1.24243 \\
\hline $\mathrm{H}$ & 0.87568 & 3.90953 & 1.80215 \\
\hline $\mathrm{H}$ & -1.81035 & 4.73015 & -0.70828 \\
\hline $\mathrm{H}$ & -0.51527 & 5.87616 & -1.08669 \\
\hline $\mathrm{H}$ & -0.79373 & 4.4756 & -2.14534 \\
\hline $\mathrm{H}$ & 1.98565 & 5.06903 & -0.81285 \\
\hline $\mathrm{H}$ & 1.64056 & 3.66872 & -1.84003 \\
\hline $\mathrm{H}$ & 2.27503 & 3.44301 & -0.17982 \\
\hline $\mathrm{H}$ & -0.52658 & 0.27864 & 0.17372 \\
\hline
\end{tabular}

\begin{tabular}{|c|c|c|c|}
\hline $\mathrm{H}$ & -1.79496 & -0.28691 & -2.33056 \\
\hline $\mathrm{H}$ & 0.01025 & -1.7732 & -3.25065 \\
\hline $\mathrm{H}$ & 0.2531 & -0.0387 & -3.40458 \\
\hline $\mathrm{H}$ & 1.58066 & -2.36361 & -0.32281 \\
\hline $\mathrm{H}$ & 1.99559 & 1.48536 & -3.46211 \\
\hline $\mathrm{H}$ & 3.7222 & 1.09336 & -3.7474 \\
\hline $\mathrm{H}$ & 2.4501 & 0.19669 & -4.61197 \\
\hline $\mathrm{H}$ & 4.7894 & -0.17607 & -2.0751 \\
\hline $\mathrm{H}$ & 4.78946 & -3.9818 & -0.94161 \\
\hline $\mathrm{H}$ & 5.88722 & -2.63894 & -1.3725 \\
\hline $\mathrm{H}$ & 6.03032 & -3.443 & 0.20625 \\
\hline $\mathrm{H}$ & 9.11102 & -0.67516 & -3.26532 \\
\hline $\mathrm{H}$ & 8.44367 & 0.76751 & -4.09184 \\
\hline $\mathrm{H}$ & 9.26862 & 0.90865 & -2.51586 \\
\hline $\mathrm{H}$ & 6.40974 & -0.59488 & -0.49855 \\
\hline $\mathrm{H}$ & 7.99574 & -0.80809 & 1.30755 \\
\hline $\mathrm{H}$ & 7.58822 & 0.87456 & 1.04213 \\
\hline $\mathrm{H}$ & 7.72469 & -0.51291 & 3.77824 \\
\hline $\mathrm{H}$ & 8.88536 & 0.64832 & 3.14668 \\
\hline $\mathrm{H}$ & 7.24617 & 1.73263 & 4.73357 \\
\hline $\mathrm{H}$ & 7.14159 & 2.42431 & 3.11957 \\
\hline $\mathrm{H}$ & 4.84712 & 1.967 & 3.98083 \\
\hline $\mathrm{H}$ & 5.27742 & 0.28404 & 4.2893 \\
\hline $\mathrm{H}$ & 3.43712 & -0.638 & 2.06401 \\
\hline $\mathrm{H}$ & 1.16492 & -0.40423 & 1.98082 \\
\hline $\mathrm{H}$ & 0.50668 & 1.26275 & 1.90876 \\
\hline $\mathrm{H}$ & 0.93329 & 0.54184 & 3.46318 \\
\hline $\mathrm{H}$ & -3.27885 & -1.81847 & -2.87541 \\
\hline $\mathrm{H}$ & -1.92692 & -2.7866 & -3.51979 \\
\hline $\mathrm{H}$ & -3.65406 & -4.43122 & -3.73669 \\
\hline $\mathrm{H}$ & -4.57501 & -3.75193 & -2.37777 \\
\hline $\mathrm{H}$ & -3.57409 & -5.67207 & -1.31869 \\
\hline $\mathrm{H}$ & -2.03587 & -5.44038 & -2.17805 \\
\hline $\mathrm{H}$ & -1.48099 & -4.32212 & -0.13848 \\
\hline $\mathrm{H}$ & -2.02749 & -2.97948 & 3 1.63345 \\
\hline $\mathrm{H}$ & -2.27151 & -1.51025 & 3.52227 \\
\hline $\mathrm{H}$ & -2.29466 & -3.14754 & 4.2176 \\
\hline $\mathrm{H}$ & -3.51421 & -1.95431 & 4.70468 \\
\hline $\mathrm{H}$ & -4.1054 & -4.7735 & 3.60881 \\
\hline $\mathrm{H}$ & -5.37456 & -3.60439 & 4.05975 \\
\hline $\mathrm{H}$ & -5.38063 & -4.33139 & $9 \quad 2.44663$ \\
\hline $\mathrm{H}$ & -3.32647 & -0.98689 & 91.00092 \\
\hline $\mathrm{H}$ & -5.91069 & 0.39534 & 4 1.36004 \\
\hline $\mathrm{H}$ & -6.40147 & -0.76558 & $\begin{array}{ll}8 & -2.76887\end{array}$ \\
\hline $\mathrm{H}$ & -7.64118 & 0.51617 & $7-2.58357$ \\
\hline $\mathrm{H}$ & -5.95036 & 0.94454 & $4-3.00078$ \\
\hline $\mathrm{H}$ & -4.07971 & 1.83319 & 2.20259 \\
\hline $\mathrm{H}$ & -3.18796 & 1.65138 & 0.69126 \\
\hline $\mathrm{H}$ & -6.22353 & 3.17725 & 2.23418 \\
\hline $\mathrm{H}$ & -7.42834 & 5.11262 & 21.27596 \\
\hline $\mathrm{H}$ & -6.8065 & 5.98495 & -0.96816 \\
\hline $\mathrm{H}$ & -4.96471 & 4.90202 & $2-2.24209$ \\
\hline $\mathrm{H}$ & -3.7644 & 2.96301 & -1.28221 \\
\hline $\mathrm{H}$ & 5.72968 & -1.25773 & 2.20816 \\
\hline
\end{tabular}




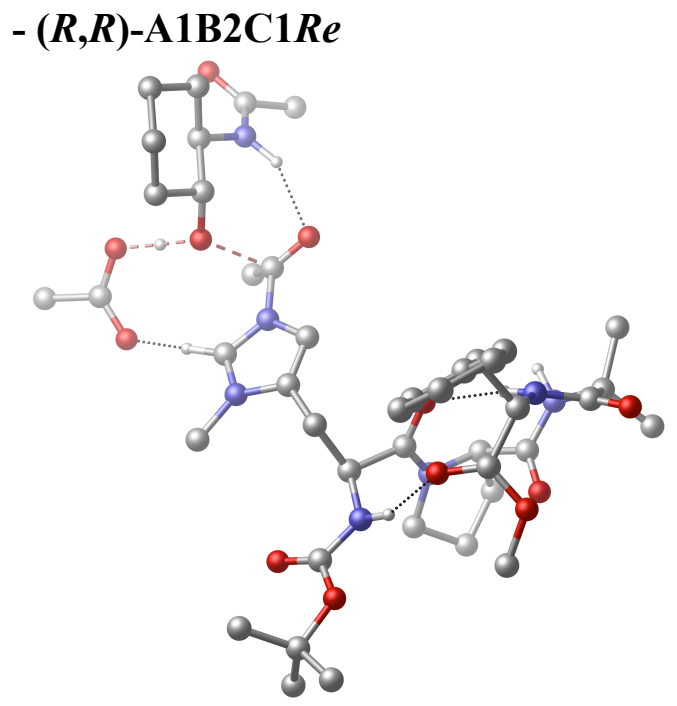




$\begin{array}{llll}\mathrm{H} & 4.9677 & 0.60857 & 2.85428 \\ \mathrm{H} & 6.44656 & -0.26458 & 4.70676 \\ \mathrm{H} & 5.46694 & -1.54542 & 4.00255 \\ \mathrm{H} & 8.40596 & -0.97169 & 3.34577 \\ \mathrm{H} & 7.80231 & -2.34753 & 4.2606 \\ \mathrm{H} & 6.56672 & -3.17989 & 2.26418 \\ \mathrm{H} & 8.27313 & -2.97297 & 1.8633 \\ \mathrm{H} & 7.81849 & -0.81807 & 0.77706 \\ \mathrm{H} & 4.88107 & -1.37561 & 1.39573 \\ \mathrm{H} & 5.79172 & -2.35261 & -0.58321 \\ \mathrm{H} & 6.25061 & -3.51069 & -2.43393 \\ \mathrm{H} & 7.61153 & -4.62194 & -2.1407 \\ \mathrm{H} & 7.87192 & -3.16356 & -3.09163 \\ \mathrm{H} & -2.89086 & 2.39917 & -2.81016 \\ \mathrm{H} & -1.46369 & 2.23799 & -3.85787 \\ \mathrm{H} & -3.24221 & 1.8928 & -5.48305 \\ \mathrm{H} & -4.25401 & 1.06187 & -4.27857 \\ \mathrm{H} & -3.28663 & -0.82184 & -5.49627 \\ \mathrm{H} & -1.70792 & -0.02958 & -5.58139 \\ \mathrm{H} & -1.26493 & -1.29302 & -3.63451 \\ \mathrm{H} & -2.03012 & -3.16395 & -2.98556 \\ \mathrm{H} & -2.21669 & -5.13416 & -1.5643 \\ \mathrm{H} & -2.74628 & -5.55626 & -3.2135 \\ \mathrm{H} & -3.71901 & -6.0392 & -1.81041 \\ \mathrm{H} & -4.76189 & -4.44736 & -4.22728 \\ \mathrm{H} & -5.78259 & -4.86078 & -2.82308 \\ \mathrm{H} & -5.64465 & -3.17545 & -3.34626 \\ \mathrm{H} & -2.83306 & -2.32166 & -0.52246 \\ \mathrm{H} & -4.96215 & -2.94534 & 1.43734 \\ \mathrm{H} & -5.66977 & 0.66509 & -0.90272 \\ \mathrm{H} & -7.24288 & 0.39785 & -0.09081 \\ \mathrm{H} & -5.92815 & 1.27393 & 0.7626 \\ \mathrm{H} & -2.6441 & -3.54251 & 2.24402 \\ \mathrm{H} & -2.15745 & -1.85722 & 2.05198 \\ \mathrm{H} & -4.34514 & -4.18115 & 3.97212 \\ \mathrm{H} & -5.24135 & -3.7053 & 6.22705 \\ \mathrm{H} & -4.93061 & -1.45673 & 7.24435 \\ \mathrm{H} & -3.71807 & 0.30768 & 5.98038 \\ \mathrm{H} & -2.83667 & -0.17091 & 3.71426 \\ & & & \\ & & & \end{array}$

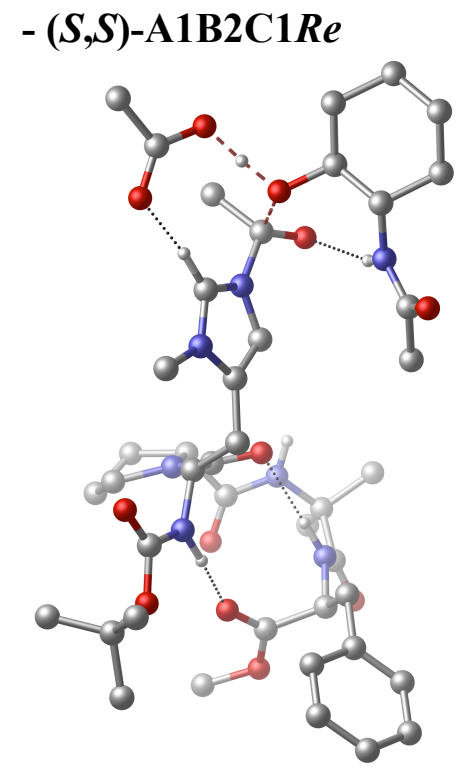

$\begin{array}{lccc}\mathrm{N} & -2.03137 & 1.86342 & -0.64128 \\ \mathrm{C} & -0.79919 & 1.16944 & -0.97129 \\ \mathrm{C} & 0.20222 & 1.15648 & 0.21271 \\ \mathrm{C} & -1.10878 & -0.27454 & -1.42079 \\ \mathrm{C} & 1.60255 & 0.84977 & -0.21093 \\ \mathrm{O} & -0.88461 & -1.23669 & -0.66869 \\ \mathrm{~N} & 2.52372 & 1.83652 & -0.57778 \\ \mathrm{C} & 2.24454 & -0.34216 & -0.39457 \\ \mathrm{C} & 3.67075 & 1.25539 & -0.95419 \\ \mathrm{~N} & 3.5164 & -0.06803 & -0.85644 \\ \mathrm{~N} & -1.59689 & -0.45315 & -2.66637 \\ \mathrm{C} & -1.9794 & 0.59312 & -3.63497 \\ \mathrm{C} & -1.73856 & -1.81739 & -3.21768 \\ \mathrm{C} & -2.77204 & -0.18801 & -4.68824 \\ \mathrm{C} & -2.08747 & -1.56385 & -4.69888 \\ \mathrm{C} & 2.33706 & 3.28874 & -0.49116 \\ \mathrm{C} & -3.23342 & -4.52302 & -0.90842 \\ \mathrm{~N} & -2.3853 & -3.63831 & -1.72579 \\ \mathrm{C} & -4.10076 & -3.71772 & 0.10137 \\ \mathrm{O} & -5.16631 & -4.16054 & 0.50433 \\ \mathrm{C} & -2.29477 & -5.43261 & -0.09315 \\ \mathrm{C} & -4.15398 & -5.3646 & -1.80423 \\ \mathrm{C} & -2.03824 & 3.22857 & -0.61069 \\ \mathrm{O} & -1.06068 & 3.9181 & -0.88677 \\ \mathrm{O} & -3.25427 & 3.67804 & -0.23927 \\ \mathrm{C} & -3.54151 & 5.12261 & -0.1413 \\ \mathrm{C} & -3.36486 & 5.78611 & -1.51062 \\ \mathrm{C} & -5.01182 & 5.13685 & 0.28174 \\ \mathrm{C} & -2.65593 & 5.75882 & 0.93392 \\ \mathrm{C} & -5.23954 & 2.25914 & 3.81034 \\ \mathrm{C} & -4.45932 & 1.44038 & 2.99186 \\ \mathrm{C} & -5.93475 & 1.71653 & 4.89114 \\ \mathrm{C} & -4.35899 & 0.06595 & 3.24529 \\ \mathrm{C} & -5.84413 & 0.34781 & 5.15124 \\ \mathrm{C} & -3.52698 & -0.83911 & 2.35917\end{array}$




\begin{tabular}{|c|c|c|c|}
\hline 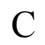 & -5.0619 & -0.46781 & 4.33475 \\
\hline $\mathrm{C}$ & -4.36434 & -1.59549 & 1.31411 \\
\hline $\mathrm{N}$ & -3.55094 & -2.54989 & 0.56506 \\
\hline C & -4.98347 & -0.65193 & 30.2765 \\
\hline $\mathrm{O}$ & -4.3899 & 0.27444 & -0.25513 \\
\hline $\mathrm{O}$ & -6.21969 & -1.01426 & $\begin{array}{ll}5 & -0.05313\end{array}$ \\
\hline $\mathrm{C}$ & -6.80017 & -0.33242 & $2-1.18437$ \\
\hline $\mathrm{O}$ & -4.03667 & -2.35305 & $\begin{array}{ll}5 & -2.65847\end{array}$ \\
\hline $\mathrm{C}$ & -2.84785 & -2.6179 & -2.49855 \\
\hline $\mathrm{C}$ & 4.54295 & -1.14299 & -1.14331 \\
\hline $\mathrm{C}$ & 5.16598 & -1.00056 & -2.51461 \\
\hline $\mathrm{O}$ & 4.31031 & -2.22418 & -0.61435 \\
\hline $\mathrm{C}$ & 8.36911 & -1.52723 & 3.15732 \\
\hline $\mathrm{C}$ & 8.92048 & -1.69687 & 1.73635 \\
\hline $\mathrm{C}$ & 6.86562 & -1.82032 & 3.19032 \\
\hline $\mathrm{C}$ & 8.15569 & -0.81473 & 0.74084 \\
\hline $\mathrm{C}$ & 6.07821 & -0.94758 & 2.19063 \\
\hline $\mathrm{C}$ & 6.64058 & -1.08413 & 0.76103 \\
\hline $\mathrm{O}$ & 5.95211 & -0.21926 & $5-0.13631$ \\
\hline $\mathrm{N}$ & 4.66186 & -1.3073 & 2.20602 \\
\hline $\mathrm{C}$ & 3.83184 & -0.9327 & 3.21324 \\
\hline $\mathrm{O}$ & 4.18791 & -0.23032 & 24.15807 \\
\hline $\mathrm{C}$ & 7.09949 & 2.29391 & -1.92082 \\
\hline $\mathrm{C}$ & 8.234 & 3.13083 & -2.4934 \\
\hline $\mathrm{O}$ & 5.93023 & 2.72398 & -1.965 \\
\hline $\mathrm{O}$ & 7.47043 & 1.1635 & -1.42111 \\
\hline $\mathrm{C}$ & 2.39956 & -1.44831 & 3.11321 \\
\hline $\mathrm{H}$ & -5.63451 & 4.6427 & -0.46919 \\
\hline $\mathrm{H}$ & -5.35839 & 6.16794 & +0.39692 \\
\hline $\mathrm{H}$ & -5.14626 & 4.61674 & 1.23428 \\
\hline $\mathrm{H}$ & -3.97801 & 5.27752 & -2.26119 \\
\hline $\mathrm{H}$ & -3.69326 & 6.82875 & -1.45599 \\
\hline $\mathrm{H}$ & -2.3223 & 5.76308 & -1.82818 \\
\hline $\mathrm{H}$ & -2.94982 & 6.803 & 1.08015 \\
\hline $\mathrm{H}$ & -1.60517 & 5.72794 & +0.64504 \\
\hline $\mathrm{H}$ & -2.77874 & 5.23593 & 1.88751 \\
\hline $\mathrm{H}$ & -2.87265 & 1.34542 & -0.38819 \\
\hline $\mathrm{H}$ & -0.33634 & 1.70477 & -1.80792 \\
\hline $\mathrm{H}$ & 0.1621 & 2.13927 & 0.68643 \\
\hline $\mathrm{H}$ & -0.12661 & 0.41143 & $\begin{array}{ll}3 & 0.93929\end{array}$ \\
\hline $\mathrm{H}$ & 1.90221 & -1.35193 & -0.25051 \\
\hline $\mathrm{H}$ & 2.38445 & 3.60644 & 0.55379 \\
\hline $\mathrm{H}$ & 3.14481 & 3.76519 & -1.047 \\
\hline $\mathrm{H}$ & 1.36849 & 3.57058 & -0.90775 \\
\hline $\mathrm{H}$ & 4.57924 & 1.78833 & -1.27881 \\
\hline $\mathrm{H}$ & 4.44099 & -1.35809 & -3.25564 \\
\hline $\mathrm{H}$ & 5.44791 & 0.02469 & -2.75192 \\
\hline $\mathrm{H}$ & 6.04939 & -1.63798 & -2.55956 \\
\hline $\mathrm{H}$ & 8.83249 & 2.52843 & -3.18267 \\
\hline $\mathrm{H}$ & 7.84346 & 4.01167 & -3.00413 \\
\hline $\mathrm{H}$ & 8.89874 & 3.44396 & -1.68168 \\
\hline $\mathrm{H}$ & 6.66284 & 0.477 & -0.77937 \\
\hline $\mathrm{H}$ & 8.52908 & -0.95749 & -0.27775 \\
\hline $\mathrm{H}$ & 8.32402 & 0.24252 & 0.9801 \\
\hline
\end{tabular}

$\begin{array}{llll}\mathrm{H} & 8.83383 & -2.75142 & 1.43789 \\ \mathrm{H} & 9.98847 & -1.45072 & 1.70168 \\ \mathrm{H} & 8.8967 & -2.18493 & 3.85835 \\ \mathrm{H} & 8.5504 & -0.49813 & 3.49748 \\ \mathrm{H} & 6.45018 & -1.65285 & 4.18809 \\ \mathrm{H} & 6.69241 & -2.87646 & 2.93941 \\ \mathrm{H} & 4.31459 & -1.85589 & 1.42615 \\ \mathrm{H} & 2.22417 & -2.10228 & 2.25491 \\ \mathrm{H} & 1.71679 & -0.59521 & 3.06138 \\ \mathrm{H} & 2.16466 & -1.99485 & 4.03062 \\ \mathrm{H} & -2.56617 & 1.37067 & -3.14331 \\ \mathrm{H} & -1.0824 & 1.05378 & -4.07077 \\ \mathrm{H} & -2.7592 & 0.30587 & -5.66301 \\ \mathrm{H} & -3.80778 & -0.30316 & -4.36091 \\ \mathrm{H} & -2.72599 & -2.34969 & -5.10607 \\ \mathrm{H} & -1.16437 & -1.53197 & -5.28742 \\ \mathrm{H} & -0.78198 & -2.33843 & -3.10622 \\ \mathrm{H} & -1.39943 & -3.61318 & -1.50351 \\ \mathrm{H} & -1.62657 & -4.8472 & 0.54742 \\ \mathrm{H} & -1.68977 & -6.05343 & -0.76169 \\ \mathrm{H} & -2.88792 & -6.08979 & 0.54631 \\ \mathrm{H} & -3.549 & -6.00498 & -2.45296 \\ \mathrm{H} & -4.80224 & -5.98295 & -1.18245 \\ \mathrm{H} & -4.78098 & -4.71877 & -2.41993 \\ \mathrm{H} & -2.71019 & -2.19065 & 0.12345 \\ \mathrm{H} & -5.1645 & -2.16662 & 1.78695 \\ \mathrm{H} & -6.21513 & -0.55531 & -2.07899 \\ \mathrm{H} & -7.80964 & -0.73073 & -1.27361 \\ \mathrm{H} & -6.82102 & 0.74549 & -1.0117 \\ \mathrm{H} & -3.01105 & -1.59212 & 2.96376 \\ \mathrm{H} & -2.75804 & -0.25768 & 1.84014 \\ \mathrm{H} & -4.98864 & -1.53155 & 4.54856 \\ \mathrm{H} & -6.37763 & -0.08378 & 5.99315 \\ \mathrm{H} & -6.53913 & 2.35475 & 5.529 \\ \mathrm{H} & -5.29992 & 3.32455 & 3.60572 \\ \mathrm{H} & -3.92659 & 1.87352 & 2.14992 \\ \mathrm{H} & 6.46226 & -2.12389 & 0.43608 \\ \mathrm{H} & 6.15859 & 0.10245 & 2.49731\end{array}$

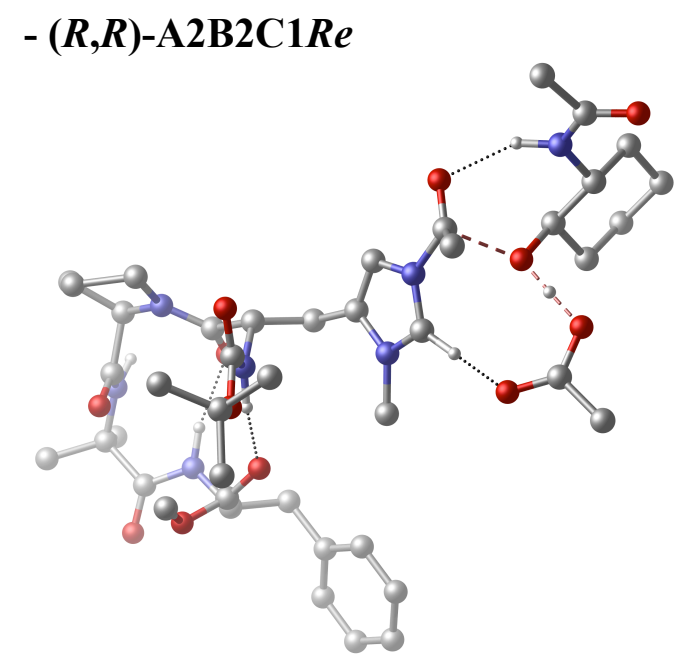




\begin{tabular}{|c|c|c|c|}
\hline $\mathrm{N}$ & 1.33308 & -1.2927 & 0.76267 \\
\hline $\mathrm{C}$ & 1.10888 & -1.6223 & -0.63423 \\
\hline $\mathrm{C}$ & 0.2248 & -0.56759 & -1.3431 \\
\hline $\mathrm{C}$ & 2.4627 & -1.73427 & -1.36233 \\
\hline $\mathrm{C}$ & -1.13962 & -0.45592 & $2-0.73783$ \\
\hline $\mathrm{O}$ & 2.95416 & -0.75743 & $3-1.945$ \\
\hline $\mathrm{N}$ & -1.50863 & 0.51139 & 90.20479 \\
\hline $\mathrm{C}$ & -2.2463 & -1.23877 & -0.91221 \\
\hline C & -2.78073 & 0.31093 & 0.57405 \\
\hline $\mathrm{N}$ & -3.23786 & -0.75604 & $\begin{array}{ll}4 & -0.08538\end{array}$ \\
\hline $\mathrm{N}$ & 3.09151 & -2.93109 & $9-1.33559$ \\
\hline $\mathrm{C}$ & 2.69494 & -4.15403 & $3-0.60911$ \\
\hline $\mathrm{C}$ & 4.3966 & -3.06762 & -1.9953 \\
\hline $\mathrm{C}$ & 4.02928 & -4.88383 & $3-0.42967$ \\
\hline $\mathrm{C}$ & 4.78777 & -4.53978 & -1.72142 \\
\hline $\mathrm{C}$ & -0.70024 & 1.62896 & 0.69446 \\
\hline $\mathrm{C}$ & 7.21778 & -0.43693 & $3-1.99811$ \\
\hline $\mathrm{N}$ & 6.13943 & -1.39004 & $4-2.30369$ \\
\hline $\mathrm{C}$ & 6.72228 & 0.77617 & -1.16178 \\
\hline $\mathrm{O}$ & 7.5362 & 1.47587 & -0.57229 \\
\hline $\mathrm{C}$ & 7.72427 & 0.1256 & -3.33995 \\
\hline $\mathrm{C}$ & 8.36256 & -1.13187 & $7-1.24314$ \\
\hline $\mathrm{C}$ & 0.81358 & -2.04872 & 21.77753 \\
\hline $\mathrm{O}$ & 0.2072 & -3.09946 & 1.61321 \\
\hline $\mathrm{O}$ & 1.0824 & -1.45589 & 2.96357 \\
\hline $\mathrm{C}$ & 0.60059 & -2.04241 & 1 4.23244 \\
\hline $\mathrm{C}$ & 1.23053 & -3.42194 & 4.44438 \\
\hline $\mathrm{C}$ & 1.11115 & -1.04422 & 5.27355 \\
\hline $\mathrm{C}$ & -0.93019 & -2.09281 & 4.2326 \\
\hline $\mathrm{C}$ & 2.10383 & 5.61259 & 1.07558 \\
\hline $\mathrm{C}$ & 2.39662 & 4.46513 & 0.33673 \\
\hline $\mathrm{C}$ & 3.02275 & 6.66054 & 1.1354 \\
\hline $\mathrm{C}$ & 3.60742 & 4.34559 & -0.3567 \\
\hline $\mathrm{C}$ & 4.23517 & 6.55324 & 0.45141 \\
\hline $\mathrm{C}$ & 3.94692 & 3.098 & -1.14788 \\
\hline $\mathrm{C}$ & 4.52241 & 5.40608 & -0.28744 \\
\hline $\mathrm{C}$ & 4.82894 & 2.11173 & -0.36293 \\
\hline $\mathrm{N}$ & 5.38632 & 1.06338 & -1.21485 \\
\hline $\mathrm{C}$ & 4.07049 & 1.38943 & 0.75371 \\
\hline $\mathrm{O}$ & 2.86871 & 1.15949 & 0.73638 \\
\hline $\mathrm{O}$ & 4.9047 & 0.96234 & 1.69863 \\
\hline $\mathrm{C}$ & 4.38906 & 0.02249 & 2.66272 \\
\hline $\mathrm{O}$ & 5.57139 & -1.99756 & $6-0.17035$ \\
\hline $\mathrm{C}$ & 5.41789 & -2.08896 & $5-1.38461$ \\
\hline $\mathrm{C}$ & -4.63176 & -1.32251 & $1-0.03002$ \\
\hline $\mathrm{C}$ & -5.17788 & -1.42127 & $\begin{array}{ll}7 & 1.37456\end{array}$ \\
\hline $\mathrm{O}$ & -4.90071 & -2.09391 & $1-0.94089$ \\
\hline $\mathrm{C}$ & -8.10597 & 1.84015 & $5-3.48379$ \\
\hline $\mathrm{C}$ & -6.62156 & 2.22442 & -3.55165 \\
\hline $\mathrm{C}$ & -8.27668 & 0.40302 & -2.97657 \\
\hline $\mathrm{C}$ & -5.93633 & 2.01261 & -2.19491 \\
\hline $\mathrm{C}$ & -7.60246 & 0.19049 & -1.60594 \\
\hline $\mathrm{C}$ & -6.1087 & 0.57973 & -1.65967 \\
\hline $\mathrm{O}$ & -5.45871 & 0.45494 & $4-0.41136$ \\
\hline
\end{tabular}

\begin{tabular}{|c|c|c|c|}
\hline N & -7.7324 & -1.19553 & -1.16147 \\
\hline $\mathrm{C}$ & -8.90569 & -1.71039 & -0.70539 \\
\hline $\mathrm{O}$ & -9.94626 & -1.06024 & -0.63313 \\
\hline $\mathrm{C}$ & -5.49518 & 2.39047 & 2.16837 \\
\hline $\mathrm{C}$ & -6.06773 & 3.45758 & 3.09005 \\
\hline $\mathrm{O}$ & -4.32675 & 1.98376 & 2.34884 \\
\hline $\mathrm{O}$ & -6.30572 & 1.98346 & 1.25476 \\
\hline $\mathrm{C}$ & -8.85147 & -3.17675 & -0.28873 \\
\hline $\mathrm{H}$ & 2.20343 & -0.98582 & 5.25409 \\
\hline $\mathrm{H}$ & 0.80217 & -1.35787 & 6.27465 \\
\hline $\mathrm{H}$ & 0.70622 & -0.04608 & 5.08468 \\
\hline $\mathrm{H}$ & 2.32147 & -3.35604 & 4.38258 \\
\hline $\mathrm{H}$ & 0.97004 & -3.79277 & 5.44068 \\
\hline $\mathrm{H}$ & 0.87422 & -4.1345 & 3.70051 \\
\hline $\mathrm{H}$ & -1.28579 & -2.41625 & 5.21579 \\
\hline $\mathrm{H}$ & -1.2993 & -2.78945 & 3.4797 \\
\hline $\mathrm{H}$ & -1.34512 & -1.09961 & 4.03445 \\
\hline $\mathrm{H}$ & 1.87486 & -0.45922 & 0.98397 \\
\hline $\mathrm{H}$ & 0.59158 & -2.58376 & -0.64033 \\
\hline $\mathrm{H}$ & 0.74995 & 0.3902 & -1.32715 \\
\hline $\mathrm{H}$ & 0.12549 & -0.84508 & -2.39735 \\
\hline $\mathrm{H}$ & -2.42732 & -2.09284 & -1.54339 \\
\hline $\mathrm{H}$ & -0.41188 & 2.27351 & -0.13909 \\
\hline $\mathrm{H}$ & -1.31039 & 2.19925 & 1.39526 \\
\hline $\mathrm{H}$ & 0.19618 & 1.26228 & 1.19449 \\
\hline $\mathrm{H}$ & -3.37208 & 0.95177 & 1.24868 \\
\hline $\mathrm{H}$ & -4.73078 & -2.30235 & 1.85046 \\
\hline $\mathrm{H}$ & -4.9662 & -0.53758 & 1.97695 \\
\hline $\mathrm{H}$ & -6.25638 & -1.56123 & 1.30934 \\
\hline $\mathrm{H}$ & -6.99173 & 3.09198 & 3.54761 \\
\hline $\mathrm{H}$ & -5.34836 & 3.72596 & 3.86457 \\
\hline $\mathrm{H}$ & -6.33109 & 4.34448 & 2.50516 \\
\hline $\mathrm{H}$ & -5.86469 & 1.17523 & 0.4282 \\
\hline $\mathrm{H}$ & -6.35562 & 2.70486 & -1.45358 \\
\hline $\mathrm{H}$ & -4.8635 & 2.22973 & -2.25675 \\
\hline $\mathrm{H}$ & -6.50497 & 3.2673 & -3.871 \\
\hline $\mathrm{H}$ & -6.12363 & 1.6075 & -4.31369 \\
\hline $\mathrm{H}$ & -8.62926 & 2.53005 & -2.80735 \\
\hline $\mathrm{H}$ & -8.57722 & 1.95125 & -4.46771 \\
\hline $\mathrm{H}$ & -7.83065 & -0.29795 & -3.69671 \\
\hline $\mathrm{H}$ & -9.33377 & 0.14063 & -2.88121 \\
\hline $\mathrm{H}$ & -8.10483 & 0.82622 & -0.86563 \\
\hline $\mathrm{H}$ & -5.61154 & -0.12086 & -2.35254 \\
\hline $\mathrm{H}$ & -6.90247 & -1.77793 & -1.19275 \\
\hline $\mathrm{H}$ & -7.84387 & -3.60123 & -0.30916 \\
\hline $\mathrm{H}$ & -9.49514 & -3.75828 & -0.95561 \\
\hline $\mathrm{H}$ & -9.26343 & -3.26875 & 0.71967 \\
\hline $\mathrm{H}$ & 2.19778 & -3.91826 & 0.33148 \\
\hline $\mathrm{H}$ & 2.00018 & -4.7469 & -1.21997 \\
\hline $\mathrm{H}$ & 3.89924 & -5.95872 & -0.28171 \\
\hline $\mathrm{H}$ & 4.56141 & -4.46782 & 0.42955 \\
\hline $\mathrm{H}$ & 5.8691 & -4.66759 & -1.63251 \\
\hline $\mathrm{H}$ & 4.44247 & -5.1673 & -2.55009 \\
\hline $\mathrm{H}$ & 4.28416 & -2.86854 & -3.06717 \\
\hline
\end{tabular}




\begin{tabular}{|c|c|c|}
\hline $\mathrm{H}$ & $5.85393-1.46598$ & -3.26902 \\
\hline $\mathrm{H}$ & $6.91601 \quad 0.61064$ & -3.89833 \\
\hline $\mathrm{H}$ & $8.15026-0.67462$ & -3.95425 \\
\hline $\mathrm{H}$ & $8.50046 \quad 0.86898$ & -3.1502 \\
\hline $\mathrm{H}$ & $8.79368-1.91659$ & -1.87221 \\
\hline $\mathrm{H}$ & $9.13082-0.39855$ & -0.99632 \\
\hline $\mathrm{H}$ & $7.9937-1.57659$ & -0.31824 \\
\hline $\mathrm{H}$ & $\begin{array}{ll}4.73556 & 0.39775\end{array}$ & -1.62253 \\
\hline $\mathrm{H}$ & $5.68718 \quad 2.62528$ & 0.07538 \\
\hline $\mathrm{H}$ & $4.29513-0.95673$ & 2.18731 \\
\hline $\mathrm{H}$ & $5.13322-0.01556$ & 3.45704 \\
\hline $\mathrm{H}$ & $3.42229 \quad 0.3485$ & 3.04861 \\
\hline $\mathrm{H}$ & $4.50482 \quad 3.37113$ & -2.04923 \\
\hline $\mathrm{H}$ & 3.037192 .58206 & -1.46955 \\
\hline $\mathrm{H}$ & $5.46648 \quad 5.33287$ & -0.82222 \\
\hline $\mathrm{H}$ & 7.36501 & 0.48921 \\
\hline $\mathrm{H}$ & $2.79551 \quad 7.55483$ & 1.70808 \\
\hline $\mathrm{H}$ & $1.1571 \quad 5.68858$ & 1.60299 \\
\hline $\mathrm{H}$ & $1.68466 \quad 3.64636$ & 0.30011 \\
\hline
\end{tabular}

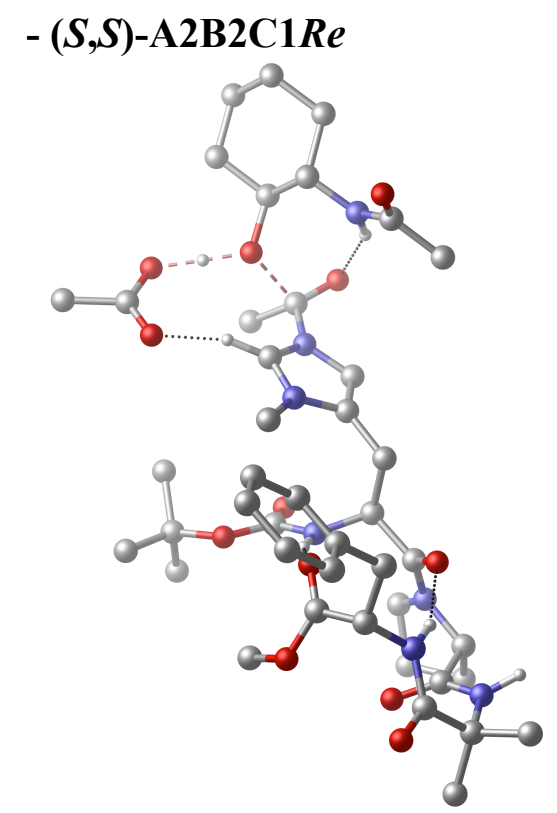

$\begin{array}{llll}\mathrm{N} & 1.12474 & -1.45402 & 0.40512 \\ \mathrm{C} & 1.03542 & -1.48413 & -1.04511 \\ \mathrm{C} & 0.17237 & -0.32271 & -1.60256 \\ \mathrm{C} & 2.45496 & -1.43052 & -1.63879 \\ \mathrm{C} & -1.24225 & -0.41544 & -1.12208 \\ \mathrm{O} & 2.95358 & -0.36278 & -2.01965 \\ \mathrm{~N} & -1.7261 & 0.20611 & 0.03225 \\ \mathrm{C} & -2.27544 & -1.18285 & -1.58369 \\ \mathrm{C} & -2.98923 & -0.18466 & 0.25171 \\ \mathrm{~N} & -3.33864 & -1.03337 & -0.71539 \\ \mathrm{~N} & 3.13448 & -2.59794 & -1.71917 \\ \mathrm{C} & 2.72046 & -3.92364 & -1.21942 \\ \mathrm{C} & 4.50485 & -2.59588 & -2.246 \\ \mathrm{C} & 4.05854 & -4.64421 & -1.02677\end{array}$

\begin{tabular}{|c|c|c|c|}
\hline $\mathrm{C}$ & 4.9216 & -4.08377 & -2.16833 \\
\hline $\mathrm{C}$ & -1.06142 & 1.21803 & 0.8581 \\
\hline $\mathrm{C}$ & 7.19201 & 0.07169 & -1.50811 \\
\hline $\mathrm{N}$ & 6.19828 & -0.84688 & -2.0855 \\
\hline C & 6.55494 & 1.10127 & -0.53328 \\
\hline $\mathrm{O}$ & 7.26768 & 1.70536 & 0.25823 \\
\hline $\mathrm{C}$ & 7.80045 & 0.87711 & -2.67187 \\
\hline $\mathrm{C}$ & 8.28938 & -0.70874 & -0.76632 \\
\hline $\mathrm{C}$ & 0.37655 & -2.29078 & 1.19164 \\
\hline $\mathrm{O}$ & -0.23926 & -3.26078 & 0.77073 \\
\hline $\mathrm{O}$ & 0.44453 & -1.87475 & 2.47642 \\
\hline $\mathrm{C}$ & -0.25351 & -2.60506 & 3.55818 \\
\hline $\mathrm{C}$ & 0.29926 & -4.02963 & 3.65946 \\
\hline $\mathrm{C}$ & 0.11391 & -1.78709 & 4.79776 \\
\hline $\mathrm{C}$ & -1.76645 & -2.58097 & 3.3209 \\
\hline $\mathrm{C}$ & 1.4273 & 5.29993 & 1.92084 \\
\hline $\mathrm{C}$ & 1.87761 & 4.31845 & 1.0366 \\
\hline $\mathrm{C}$ & 2.27109 & 6.34633 & 2.29419 \\
\hline $\mathrm{C}$ & 3.1734 & 4.36674 & 0.50824 \\
\hline $\mathrm{C}$ & 3.56664 & 6.40503 & 1.77702 \\
\hline $\mathrm{C}$ & 3.67954 & 3.29839 & -0.44021 \\
\hline $\mathrm{C}$ & 4.01145 & 5.42364 & 0.89194 \\
\hline C & 4.52515 & 2.22285 & 0.26307 \\
\hline $\mathrm{N}$ & 5.22076 & 1.35748 & -0.68705 \\
\hline $\mathrm{C}$ & 3.69298 & 1.29248 & 1.14953 \\
\hline $\mathrm{O}$ & 2.5173 & 1.01864 & 0.95043 \\
\hline $\mathrm{O}$ & 4.43973 & 0.7466 & 2.10656 \\
\hline C & 3.87351 & -0.36208 & 2.83418 \\
\hline $\mathrm{O}$ & 5.43923 & -1.81451 & -0.15525 \\
\hline C & 5.41645 & -1.70784 & -1.3778 \\
\hline C & -4.65215 & -1.77934 & -0.89264 \\
\hline C & -4.88665 & -2.80015 & 0.19834 \\
\hline $\mathrm{O}$ & -4.98767 & -1.9468 & -2.05848 \\
\hline C & -9.04321 & 1.86964 & -1.66187 \\
\hline C & -9.31976 & 0.64989 & -0.77359 \\
\hline C & -7.84231 & 1.61331 & -2.58031 \\
\hline C & -8.07169 & 0.25866 & 0.02914 \\
\hline C & -6.58108 & 1.21365 & -1.78718 \\
\hline C & -6.8573 & 0.0001 & -0.87784 \\
\hline $\mathrm{O}$ & -5.70401 & -0.32543 & -0.10956 \\
\hline $\mathrm{N}$ & -5.47168 & 0.91333 & -2.68828 \\
\hline C & -4.73767 & 1.88751 & -3.28477 \\
\hline $\mathrm{O}$ & -4.941 & $3.0887-$ & 3.11622 \\
\hline $\mathrm{C}$ & -5.24406 & 0.03987 & 3.10733 \\
\hline $\mathrm{C}$ & -5.72525 & 0.08373 & 4.55048 \\
\hline $\mathrm{O}$ & -4.11648 & 0.48555 & 2.8149 \\
\hline $\mathrm{O}$ & -6.08807 & -0.47812 & 2.2784 \\
\hline C & -3.63019 & 1.39529 & -4.21143 \\
\hline $\mathrm{H}$ & 1.19693 & -1.77989 & 4.95302 \\
\hline $\mathrm{H}$ & -0.35789 & -2.22081 & 5.68405 \\
\hline $\mathrm{H}$ & -0.23136 & -0.75473 & 4.69513 \\
\hline $\mathrm{H}$ & 1.38762 & -4.00893 & 3.77588 \\
\hline $\mathrm{H}$ & -0.12543 & -4.52426 & 4.53859 \\
\hline $\mathrm{H}$ & 0.04744 & -4.61252 & 2.77323 \\
\hline
\end{tabular}




\begin{tabular}{|c|c|c|}
\hline $\mathrm{H}$ & $\begin{array}{ll}-2.27025 & -3.05857\end{array}$ & 4.16748 \\
\hline $\mathrm{H}$ & $-2.02666-3.12555$ & 2.41269 \\
\hline $\mathrm{H}$ & $-2.14171-1.55542$ & 3.24707 \\
\hline $\mathrm{H}$ & $1.59822-0.6618$ & 0.83479 \\
\hline $\mathrm{H}$ & $0.55507-2.43138$ & -1.30061 \\
\hline $\mathrm{H}$ & $0.63522 \quad 0.62592$ & -1.32101 \\
\hline $\mathrm{H}$ & $0.18633-0.36323$ & -2.69607 \\
\hline $\mathrm{H}$ & $-2.34656-1.83232$ & -2.44042 \\
\hline $\mathrm{H}$ & $\begin{array}{ll}-0.89539 & 2.12407\end{array}$ & 0.27041 \\
\hline $\mathrm{H}$ & $-1.72142 \quad 1.4443$ & 1.69592 \\
\hline $\mathrm{H}$ & $\begin{array}{ll}-0.10926 & 0.84447\end{array}$ & 1.23057 \\
\hline $\mathrm{H}$ & $\begin{array}{ll}-3.58932 & 0.1422\end{array}$ & 1.10918 \\
\hline $\mathrm{H}$ & $-4.19167 \quad-3.63341$ & 0.04097 \\
\hline $\mathrm{H}$ & $-4.74502-2.3932$ & 1.19875 \\
\hline $\mathrm{H}$ & $\begin{array}{ll}-5.90674 & -3.17441\end{array}$ & 0.10226 \\
\hline $\mathrm{H}$ & $-6.08178-0.90396$ & 4.85649 \\
\hline $\mathrm{H}$ & $\begin{array}{ll}-4.92717 & 0.41924\end{array}$ & 5.21372 \\
\hline $\mathrm{H}$ & $\begin{array}{ll}-6.57559 & 0.76903\end{array}$ & 4.62799 \\
\hline $\mathrm{H}$ & $-5.87458 \quad-0.38544$ & 1.04735 \\
\hline $\mathrm{H}$ & $-8.25834-0.63119$ & 0.6387 \\
\hline $\mathrm{H}$ & $\begin{array}{lll}-7.818 & 1.0631\end{array}$ & 0.73075 \\
\hline $\mathrm{H}$ & $\begin{array}{ll}-9.63143 & -0.19486\end{array}$ & -1.40417 \\
\hline $\mathrm{H}$ & $-10.15193 \quad 0.8499$ & $9-0.08829$ \\
\hline $\mathrm{H}$ & $-9.92771 \quad 2.12012$ & -2.25953 \\
\hline $\mathrm{H}$ & $\begin{array}{ll}-8.8384 & 2.74259\end{array}$ & -1.02653 \\
\hline $\mathrm{H}$ & $\begin{array}{ll}-7.60327 & 2.49836\end{array}$ & -3.17694 \\
\hline $\mathrm{H}$ & $-8.08244 \quad 0.80304$ & -3.28298 \\
\hline $\mathrm{H}$ & $-5.23075-0.06404$ & -2.8198 \\
\hline $\mathrm{H}$ & $\begin{array}{ll}-3.50378 & 0.30931\end{array}$ & -4.20697 \\
\hline $\mathrm{H}$ & $\begin{array}{ll}-2.69021 & 1.87207\end{array}$ & -3.92075 \\
\hline $\mathrm{H}$ & $\begin{array}{ll}-3.85606 & 1.72093\end{array}$ & -5.23117 \\
\hline $\mathrm{H}$ & $2.13852-3.83719$ & -0.30206 \\
\hline $\mathrm{H}$ & $2.10047-4.43416$ & -1.96918 \\
\hline $\mathrm{H}$ & $3.95446-5.73151$ & -1.06162 \\
\hline $\mathrm{H}$ & $4.49271 \quad-4.35865$ & -0.06546 \\
\hline $\mathrm{H}$ & $5.99376-4.20176$ & -1.9947 \\
\hline $\mathrm{H}$ & $\begin{array}{lll}4.67807 & -4.57839\end{array}$ & -3.11483 \\
\hline $\mathrm{H}$ & $4.49701 \quad-2.22668$ & -3.27773 \\
\hline $\mathrm{H}$ & $6.01698-0.76438$ & -3.07524 \\
\hline $\mathrm{H}$ & $\begin{array}{ll}7.02893 & 1.42787\end{array}$ & -3.22109 \\
\hline $\mathrm{H}$ & $8.324 \quad 0.21133$ & -3.36615 \\
\hline $\mathrm{H}$ & $8.51693 \quad 1.59919$ & -2.27622 \\
\hline $\mathrm{H}$ & $8.81858-1.35736$ & -1.47111 \\
\hline $\mathrm{H}$ & $8.99298-0.007$ & -0.31744 \\
\hline $\mathrm{H}$ & $7.85383-1.31939$ & 0.02543 \\
\hline $\mathrm{H}$ & $4.649 \quad 0.76089$ & -1.27847 \\
\hline $\mathrm{H}$ & $5.30469 \quad 2.68081$ & 0.87527 \\
\hline $\mathrm{H}$ & $3.87901-1.24659$ & 2.1926 \\
\hline $\mathrm{H}$ & $4.53082-0.51364$ & 3.68915 \\
\hline $\mathrm{H}$ & $2.85633-0.13819$ & 3.15947 \\
\hline $\mathrm{H}$ & $4.31946 \quad 3.75201$ & -1.20394 \\
\hline $\mathrm{H}$ & $2.84648 \quad 2.81288$ & -0.95768 \\
\hline $\mathrm{H}$ & $5.01965 \quad 5.47993$ & 0.48806 \\
\hline $\mathrm{H}$ & $4.22957 \quad 7.21809$ & 2.05863 \\
\hline
\end{tabular}

$\begin{array}{lrrc}\mathrm{H} & 1.92124 & 7.11236 & 2.97988 \\ \mathrm{H} & 0.41651 & 5.24876 & 2.31548 \\ \mathrm{H} & 1.22318 & 3.50003 & 0.75307 \\ \mathrm{H} & -7.08112 & -0.85866 & -1.53346 \\ \mathrm{H} & -6.27745 & 2.05946 & -1.15809\end{array}$

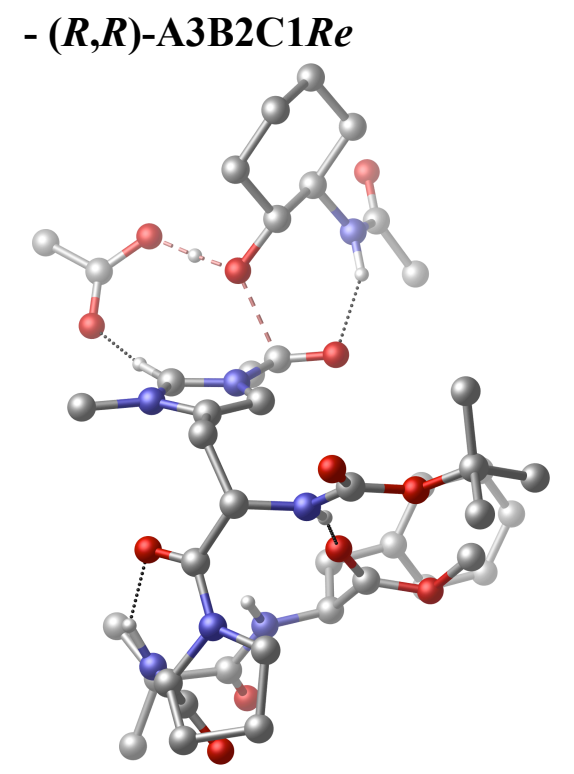

$\begin{array}{llll}\mathrm{N} & -2.24402 & 2.52386 & -0.38604 \\ \mathrm{C} & -2.35425 & 2.26744 & -1.80969 \\ \mathrm{C} & -0.98909 & 2.30841 & -2.53799 \\ \mathrm{C} & -3.14035 & 0.98459 & -2.13845 \\ \mathrm{C} & 0.01358 & 1.2668 & -2.14148 \\ \mathrm{O} & -2.64024 & 0.11316 & -2.86887 \\ \mathrm{~N} & 0.5389 & 0.32922 & -3.03817 \\ \mathrm{C} & 0.64669 & 1.03276 & -0.95281 \\ \mathrm{C} & 1.44106 & -0.43338 & -2.41221 \\ \mathrm{~N} & 1.50685 & -0.0323 & -1.13904 \\ \mathrm{~N} & -4.4093 & 0.87655 & -1.67738 \\ \mathrm{C} & -5.16467 & 1.88032 & -0.89211 \\ \mathrm{C} & -5.29307 & -0.19263 & -2.20635 \\ \mathrm{C} & -6.56113 & 1.25887 & -0.75496 \\ \mathrm{C} & -6.70138 & 0.38285 & -2.00788 \\ \mathrm{C} & 0.18869 & 0.16803 & -4.44864 \\ \mathrm{C} & -3.71217 & -3.61345 & -1.37695 \\ \mathrm{~N} & -4.06438 & -2.28189 & -1.89954 \\ \mathrm{C} & -3.28361 & -3.54736 & 0.11592 \\ \mathrm{O} & -3.41088 & -4.50555 & 0.86941 \\ \mathrm{C} & -2.48923 & -4.10546 & -2.17612 \\ \mathrm{C} & -4.87936 & -4.59937 & -1.53028 \\ \mathrm{C} & -2.28182 & 3.81855 & 0.06069 \\ \mathrm{O} & -2.46193 & 4.78071 & -0.67276 \\ \mathrm{O} & -2.10587 & 3.85592 & 1.40189 \\ \mathrm{C} & -2.04402 & 5.15278 & 2.1129 \\ \mathrm{C} & -3.3835 & 5.88319 & 1.98102 \\ \mathrm{C} & -1.79633 & 4.73227 & 3.56284 \\ \mathrm{C} & -0.8692 & 5.9822 & 1.58561\end{array}$ 


\begin{tabular}{|c|c|c|c|}
\hline $\mathrm{C}$ & 1.44921 & -1.72615 & 4.90201 \\
\hline $\mathrm{C}$ & 0.89295 & -1.83714 & 3.62589 \\
\hline $\mathrm{C}$ & 0.98193 & -2.5275 & 5.94445 \\
\hline $\mathrm{C}$ & -0.13823 & -2.75342 & 3.37621 \\
\hline $\mathrm{C}$ & -0.0431 & -3.44482 & 5.70401 \\
\hline $\mathrm{C}$ & -0.75264 & -2.87359 & 2.00057 \\
\hline $\mathrm{C}$ & -0.5966 & -3.55721 & 4.42912 \\
\hline $\mathrm{C}$ & -2.15255 & -2.19983 & 1.8319 \\
\hline $\mathrm{N}$ & -2.66634 & -2.3949 & 0.4913 \\
\hline C & -2.05252 & -0.70431 & 2.06845 \\
\hline $\mathrm{O}$ & -1.84265 & 0.09625 & 1.16726 \\
\hline $\mathrm{O}$ & -2.17362 & -0.38023 & 33.35001 \\
\hline $\mathrm{C}$ & -1.92181 & 0.99782 & 3.69854 \\
\hline $\mathrm{O}$ & -5.83174 & -1.85046 & $5-0.51113$ \\
\hline $\mathrm{C}$ & -5.09878 & -1.52562 & -1.43949 \\
\hline $\mathrm{C}$ & 2.48171 & -0.51138 & -0.11068 \\
\hline $\mathrm{C}$ & 2.79497 & -1.98589 & -0.19364 \\
\hline $\mathrm{O}$ & 2.49635 & 0.1435 & 0.92634 \\
\hline $\mathrm{C}$ & 7.31149 & 2.75328 & -0.75556 \\
\hline $\mathrm{C}$ & 6.11989 & 3.17781 & -1.62438 \\
\hline $\mathrm{C}$ & 6.84212 & 1.96162 & 0.4718 \\
\hline $\mathrm{C}$ & 5.2722 & 1.96284 & -2.02386 \\
\hline $\mathrm{C}$ & 6.0017 & 0.73051 & 0.07467 \\
\hline $\mathrm{C}$ & 4.80564 & 1.14001 & -0.81016 \\
\hline $\mathrm{O}$ & 4.04231 & 0.03597 & -1.24843 \\
\hline $\mathrm{N}$ & 5.51099 & 0.01521 & 1.25151 \\
\hline $\mathrm{C}$ & 6.32134 & -0.75228 & 2.02959 \\
\hline $\mathrm{O}$ & 7.52559 & -0.88368 & 1.82358 \\
\hline $\mathrm{C}$ & 4.57024 & -2.12988 & -3.6025 \\
\hline C & 5.4267 & -2.91592 & -4.58524 \\
\hline $\mathrm{O}$ & 3.32406 & -2.23082 & -3.65838 \\
\hline $\mathrm{O}$ & 5.23657 & -1.41844 & -2.76356 \\
\hline C & 5.63511 & -1.44373 & 3.20314 \\
\hline $\mathrm{H}$ & -2.62425 & 4.12509 & 3.9404 \\
\hline $\mathrm{H}$ & -1.7073 & 5.61891 & 4.19694 \\
\hline $\mathrm{H}$ & -0.87 & $4.1567 \quad 3$ & 3.64675 \\
\hline $\mathrm{H}$ & -4.20109 & 5.24353 & 2.32846 \\
\hline $\mathrm{H}$ & -3.3721 & 6.78394 & 2.60286 \\
\hline $\mathrm{H}$ & -3.57223 & 6.17194 & 0.94723 \\
\hline $\mathrm{H}$ & -0.76059 & 6.88586 & 2.19336 \\
\hline $\mathrm{H}$ & -1.0276 & 6.27521 & 0.54793 \\
\hline $\mathrm{H}$ & 0.06244 & 5.41237 & 1.65839 \\
\hline $\mathrm{H}$ & -2.0338 & 1.7714 & 0.26471 \\
\hline $\mathrm{H}$ & -2.9297 & 3.10967 & -2.21326 \\
\hline $\mathrm{H}$ & -1.17128 & 2.24958 & -3.61314 \\
\hline $\mathrm{H}$ & -0.57646 & 3.30378 & -2.34337 \\
\hline $\mathrm{H}$ & 0.57248 & 1.51541 & 0.00588 \\
\hline $\mathrm{H}$ & 0.43799 & 1.07565 & -5.00356 \\
\hline $\mathrm{H}$ & 0.77212 & -0.66217 & -4.8471 \\
\hline $\mathrm{H}$ & -0.87883 & -0.04004 & +-4.52816 \\
\hline $\mathrm{H}$ & 2.10074 & -1.1785 & -2.88462 \\
\hline $\mathrm{H}$ & 1.97985 & -2.54005 & 0.28609 \\
\hline $\mathrm{H}$ & 2.92398 & -2.34385 & -1.21522 \\
\hline $\mathrm{H}$ & 3.71376 & -2.1624 & 0.36469 \\
\hline
\end{tabular}

$\begin{array}{llll}\mathrm{H} & 6.07387 & -3.60563 & -4.03456 \\ \mathrm{H} & 4.80181 & -3.47454 & -5.28297 \\ \mathrm{H} & 6.08277 & -2.23289 & -5.13316 \\ \mathrm{H} & 4.59839 & -0.69498 & -1.96796 \\ \mathrm{H} & 5.85291 & 1.30087 & -2.67879 \\ \mathrm{H} & 4.3863 & 2.26974 & -2.59213 \\ \mathrm{H} & 6.46362 & 3.70652 & -2.52179 \\ \mathrm{H} & 5.49822 & 3.88958 & -1.0621 \\ \mathrm{H} & 7.9919 & 2.13071 & -1.35277 \\ \mathrm{H} & 7.88858 & 3.63041 & -0.43935 \\ \mathrm{H} & 6.23019 & 2.60889 & 1.11654 \\ \mathrm{H} & 7.69057 & 1.61773 & 1.06931 \\ \mathrm{H} & 6.63846 & 0.04066 & -0.49383 \\ \mathrm{H} & 4.14023 & 1.76649 & -0.19074 \\ \mathrm{H} & 4.51886 & 0.06867 & 1.45391 \\ \mathrm{H} & 4.54723 & -1.33121 & 3.20761 \\ \mathrm{H} & 6.03848 & -1.03974 & 4.13671 \\ \mathrm{H} & 5.88638 & -2.50715 & 3.17633 \\ \mathrm{H} & -4.68461 & 2.05547 & 0.07098 \\ \mathrm{H} & -5.19537 & 2.83436 & -1.43387 \\ \mathrm{H} & -7.33842 & 2.02264 & -0.67268 \\ \mathrm{H} & -6.60299 & 0.62116 & 0.13119 \\ \mathrm{H} & -7.44262 & -0.4073 & -1.8839 \\ \mathrm{H} & -6.9715 & 0.98885 & -2.87942 \\ \mathrm{H} & -5.04195 & -0.34886 & -3.25943 \\ \mathrm{H} & -3.45822 & -1.84353 & -2.58588 \\ \mathrm{H} & -1.64829 & -3.40942 & -2.09005 \\ \mathrm{H} & -2.74715 & -4.21558 & -3.23431 \\ \mathrm{H} & -2.16806 & -5.07797 & -1.79689 \\ \mathrm{H} & -5.11533 & -4.72663 & -2.59102 \\ \mathrm{H} & -4.59883 & -5.56281 & -1.1025 \\ \mathrm{H} & -5.76208 & -4.23294 & -1.00622 \\ \mathrm{H} & -2.58356 & -1.62287 & -0.15436 \\ \mathrm{H} & -2.84573 & -2.64192 & 2.55201 \\ \mathrm{H} & -2.49645 & 1.67094 & 3.0618 \\ \mathrm{H} & -2.221 & 1.08991 & 4.7412 \\ \mathrm{H} & -0.85671 & 1.21359 & 3.58859 \\ \mathrm{H} & -0.91091 & -3.92451 & 1.74065 \\ \mathrm{H} & -0.08988 & -2.43938 & 1.24534 \\ \mathrm{H} & -1.38715 & -4.28066 & 4.24422 \\ \mathrm{H} & -0.40674 & -4.0785 & 6.50785 \\ \mathrm{H} & 1.41817 & -2.44413 & 6.93561 \\ \mathrm{H} & 2.25351 & -1.01733 & 5.07784 \\ \mathrm{H} & 1.27553 & -1.21551 & 2.81892\end{array}$




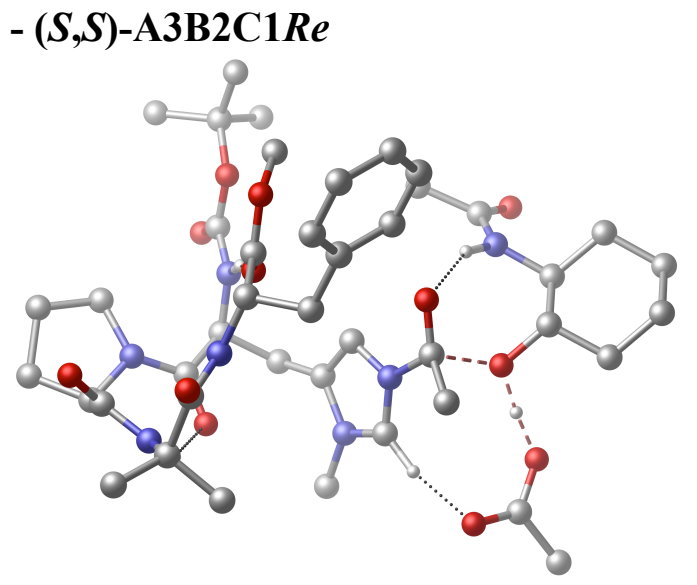

$\begin{array}{llll}\text { O } & -1.55419 & 0.24924 & 1.07198\end{array}$

$\begin{array}{llll}\mathrm{O} & -1.52611 & 0.245 & 3.32858\end{array}$

C $\quad \begin{array}{llll}-1.2025 & 1.65126 & 3.33334\end{array}$

$\begin{array}{llll}\mathrm{O} & -5.53442 & -2.03622 & -0.09957\end{array}$

C $\quad-4.80675-1.89397 \quad-1.07674$

C $\quad 2.44066-1.05391-0.05793$

$\begin{array}{llll}C & 2.70997 & -2.5427 & -0.04174\end{array}$

$\begin{array}{lllll}\mathrm{O} & 2.26894 & -0.38896 & 0.96541\end{array}$

$\begin{array}{llll}\text { C } & 7.68735 & 1.16391 & 0.79764\end{array}$

$\begin{array}{llll}\text { C } & 7.52446 & -0.36173 & 0.79023\end{array}$

$\begin{array}{llll}\text { C } & 6.35202 & 1.8553 & 1.09892\end{array}$

$\begin{array}{lllll}\text { C } & 6.43696 & -0.79465 & -0.20326\end{array}$

$\begin{array}{llll}\text { C } & 5.24836 & 1.43065 & 0.10966\end{array}$

$\begin{array}{llll}\text { C } & 5.0932 & -0.10269 & 0.07553\end{array}$

N $\quad \begin{array}{llll}-1.98908 & 2.30571 & -0.96652\end{array}$

O $\quad 4.09516 \quad-0.48224 \quad-0.86559$

$\begin{array}{lllll}\mathrm{C} & & -2.10026 & 1.74387 & -2.29911\end{array}$

$\begin{array}{llll}\text { C } & -0.73612 & 1.61645 & -3.01733\end{array}$

$\begin{array}{lllll}\text { C } & -2.89408 & 0.42345 & -2.35347\end{array}$

C $\quad 0.22293 \quad 0.61133 \quad-2.45348$

O $\quad-2.41755 \quad-0.56788 \quad-2.93189$

$\mathrm{N} \quad \begin{array}{llll}0.74726 & -0.4418 & -3.20767\end{array}$

C $\quad 0.79162 \quad 0.49635 \quad-1.21622$

C $\quad 1.58688-1.15879-2.4515$

$\mathrm{N} \quad \begin{array}{llll}1.61797 & -0.60952 & -1.23454\end{array}$

$\mathrm{N} \quad \begin{array}{llll}1.614139 & 0.40098 & -1.82609\end{array}$

$\begin{array}{llll}\text { C } & -4.87609 & 1.52499 & -1.19916\end{array}$

$\begin{array}{llll}\text { C } & -5.02919 & -0.75475 & -2.1034\end{array}$

$\begin{array}{lllll}\text { C } & -6.28225 & 0.95606 & -0.95182\end{array}$

$\begin{array}{llll}\text { C } & -6.43494 & -0.15187 & -2.00415\end{array}$

C $\quad 0.42101 \quad-0.77452 \quad-4.5929$

C $\quad-3.37318 \quad-3.89199 \quad-0.58792$

$\mathrm{N} \quad-3.7492 \quad-2.70076-1.36867$

C $\quad-2.93101 \quad-3.51754 \quad 0.85491$

$\begin{array}{llll}\text { O } & -3.04249 & -4.30443 & 1.78894\end{array}$

C $\quad-2.15058-4.52386-1.28315$

C $\quad-4.52631 \quad-4.90421 \quad-0.522$

$\begin{array}{lllll}\text { C } & -2.24434 & 3.63901 & -0.77637\end{array}$

O $\quad-2.57206 \quad 4.39983 \quad-1.67645$

$\begin{array}{lllll}\mathrm{O} & -2.09494 & 3.96305 & 0.52833\end{array}$

$\begin{array}{llll}\text { C } & -2.29069 & 5.36092 & 0.97854\end{array}$

$\begin{array}{llll}\text { C } & -3.7413 & 5.78631 & 0.73346\end{array}$

$\begin{array}{llll}\text { C } & -1.99656 & 5.27745 & 2.47737\end{array}$

$\begin{array}{lllll}\text { C } & -1.28452 & 6.28236 & 0.28284\end{array}$

$\begin{array}{llll}\text { C } & 1.6948 & -1.31517 & 5.5144\end{array}$

C $\quad \begin{array}{llll}\text { C } & 1.21895 & -1.52086 & 4.21781\end{array}$

$\begin{array}{lllll}\text { C } & 1.03561 & -1.88498 & 6.60421\end{array}$

$\begin{array}{llll}\text { C } & 0.07787 & -2.30275 & 3.99626\end{array}$

$\begin{array}{lllll}\text { C } & -0.09993 & -2.66994 & 6.39121\end{array}$

$\begin{array}{llll}\text { C } & -0.43708 & -2.53845 & 2.59461\end{array}$

$\begin{array}{llll}\text { C } & -0.57224 & -2.87975 & 5.09632\end{array}$

$\begin{array}{llll}\text { C } & -1.79568 & -1.84541 & 2.25019\end{array}$

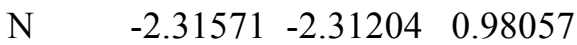

C $\quad-1.62491 \quad-0.34108 \quad 2.14208$ 


$\begin{array}{llll}\mathrm{H} & 6.44981 & 2.94362 & 1.05695 \\ \mathrm{H} & 6.02466 & 1.59757 & 2.11608 \\ \mathrm{H} & 3.23741 & 1.40467 & 0.79121 \\ \mathrm{H} & 1.69809 & 3.02015 & 1.13258 \\ \mathrm{H} & 1.71617 & 4.0251 & -0.33932 \\ \mathrm{H} & 2.3295 & 4.68598 & 1.17483 \\ \mathrm{H} & -4.38889 & 1.83269 & -0.27341 \\ \mathrm{H} & -4.89145 & 2.38751 & -1.87602 \\ \mathrm{H} & -7.05028 & 1.72984 & -1.02716 \\ \mathrm{H} & -6.33544 & 0.5096 & 0.0438 \\ \mathrm{H} & -7.17255 & -0.90129 & -1.71486 \\ \mathrm{H} & -6.71733 & 0.2673 & -2.97581 \\ \mathrm{H} & -4.79533 & -1.12772 & -3.10439 \\ \mathrm{H} & -3.16086 & -2.40967 & -2.1435 \\ \mathrm{H} & -1.32077 & -3.81332 & -1.3582 \\ \mathrm{H} & -2.41744 & -4.85889 & -2.29062 \\ \mathrm{H} & -1.80947 & -5.3894 & -0.71112 \\ \mathrm{H} & -4.77513 & -5.24341 & -1.532 \\ \mathrm{H} & -4.22452 & -5.75953 & 0.08401 \\ \mathrm{H} & -5.4075 & -4.45231 & -0.06689 \\ \mathrm{H} & -2.2794 & -1.66877 & 0.20266 \\ \mathrm{H} & -2.51279 & -2.07348 & 3.04451 \\ \mathrm{H} & -1.86865 & 2.20426 & 2.67062 \\ \mathrm{H} & -1.32059 & 1.9703 & 4.36743 \\ \mathrm{H} & -0.16813 & 1.78994 & 3.0097 \\ \mathrm{H} & -0.6243 & -3.60518 & 2.43928 \\ \mathrm{H} & 0.30477 & -2.21108 & 1.86086 \\ \mathrm{H} & -1.4474 & -3.50464 & 4.93316 \\ \mathrm{H} & -0.61297 & -3.12569 & 7.23341 \\ \mathrm{H} & 1.40732 & -1.72583 & 7.61242 \\ \mathrm{H} & 2.5849 & -0.71214 & 5.67149 \\ \mathrm{H} & 1.73974 & -1.08526 & 3.36818 \\ \mathrm{H} & 4.73702 & -0.41228 & 1.07213 \\ \mathrm{H} & 5.52214 & 1.77771 & -0.89505 \\ & & & \end{array}$

\section{- $(R, R)-\mathrm{A} 1 \mathrm{~B} 1 \mathrm{C} 2 R e$}

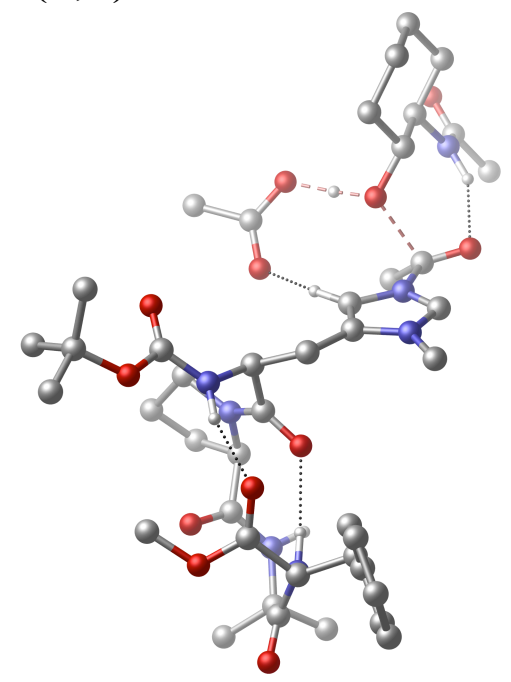

$\mathrm{N}$ $\begin{array}{lll}-1.58124 & 2.10273 \quad 0.36276\end{array}$ $\begin{array}{llll}\text { C } & -0.50418 & 1.14652 & 0.17941\end{array}$

$\begin{array}{llll}\text { C } & -0.1736 & 0.45199 & 1.51482\end{array}$

$\begin{array}{lllll}\text { C } & & -0.92857 & 0.09243 & -0.86799\end{array}$

$\begin{array}{llll}\text { C } & 0.99555 & -0.47711 & 1.41947\end{array}$

O $\quad-1.42604 \quad-0.99072-0.51164$

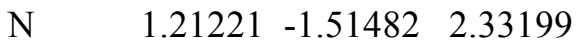

$\begin{array}{llll}\text { C } & 2.03851 & -0.51868 & 0.53636\end{array}$

$\begin{array}{llll}\mathrm{C} & 2.34681 & -2.14834 & 2.00246\end{array}$

$\begin{array}{llll}\mathrm{N} & 2.85472 & -1.56647 & 0.91806\end{array}$

$\begin{array}{llll}\mathrm{N} & -0.75709 & 0.40172 & -2.1652\end{array}$

$\begin{array}{lllll}\text { C } & -0.22016 & 1.65542 & -2.74607\end{array}$

$\begin{array}{llll}\text { C } & -1.14222 & -0.57293 & -3.19147\end{array}$

$\begin{array}{lllll}\text { C } & -0.77994 & 1.61992 & -4.17067\end{array}$

$\begin{array}{lllll}\text { C } & -0.75545 & 0.12328 & -4.51895\end{array}$

$\begin{array}{llll}\text { C } & 0.34075 & -1.87615 & 3.44609\end{array}$

C $\quad-4.31372 \quad-2.76622 \quad-3.11582$

$\mathrm{N} \quad \begin{array}{llll}\mathrm{C} & -2.9573 & -2.20018 & -3.15844\end{array}$

C $\quad-5.05819 \quad-2.43196-1.79192$

$\begin{array}{lllll}\text { O } & -6.27297 & -2.57401 & -1.72671\end{array}$

$\begin{array}{llll}\text { C } & -4.17052 & -4.29936 & -3.16841\end{array}$

$\begin{array}{llll}\text { C } & -5.15367 & -2.26828 & -4.30335\end{array}$

$\begin{array}{llll}\text { C } & -1.37951 & 3.45068 & 0.26818\end{array}$

O $\quad-0.32655 \quad 3.97226 \quad-0.07174$

$\begin{array}{lllll}\mathrm{O} & -2.52618 & 4.09864 & 0.59057\end{array}$

$\begin{array}{lllll}\mathrm{C} & -2.59538 & 5.57076 & 0.5632\end{array}$

$\begin{array}{llll}\text { C } & -2.34158 & 6.08206 & -0.85828\end{array}$

$\begin{array}{lllll}\text { C } & & -4.03974 & 5.84285 & 0.98984\end{array}$

$\begin{array}{llll}\text { C } & -1.61052 & 6.16027 & 1.57801\end{array}$

$\begin{array}{llll}\text { C } & -5.46529 & -0.80873 & 5.07576\end{array}$

$\begin{array}{llll}\text { C } & -4.73353 & -1.0462 & 3.91126\end{array}$

$\begin{array}{llll}\text { C } & -6.58046 & -1.59149 & 5.37674\end{array}$

$\begin{array}{llll}\text { C } & -5.10169 & -2.07081 & 3.02923\end{array}$

$\begin{array}{llll}\text { C } & -6.95951 & -2.61359 & 4.50473\end{array}$

$\begin{array}{llll}\text { C } & -4.32625 & -2.33476 & 1.75372\end{array}$

$\begin{array}{llll}\text { C } & -6.22476 & -2.84972 & 3.34304\end{array}$

$\begin{array}{llll}\text { C } & -4.90668 & -1.62349 & 0.51878\end{array}$

$\begin{array}{llll}\mathrm{N} & -4.28412 & -2.08926 & -0.71804\end{array}$

$\begin{array}{llll}\text { C } & -4.69733 & -0.10654 & 0.55436\end{array}$

$\begin{array}{lllll}\mathrm{O} & -3.80652 & 0.45105 & 1.17813\end{array}$

$\begin{array}{lllll}\text { O } & -5.5539 & 0.51274 & -0.25501\end{array}$

$\begin{array}{lllll}C & -5.28627 & 1.89536 & -0.56867\end{array}$

$\begin{array}{lllll}\mathrm{O} & -3.48612 & 0.02418 & -3.04859\end{array}$

$\begin{array}{llll}\text { C } & -2.65049 & -0.87187 & -3.11429\end{array}$

$\begin{array}{llll}\text { C } & 4.14542 & -2.0382 & 0.29695\end{array}$

C $\quad 4.12333 \quad-2.01864-1.21019$

$\begin{array}{lllll}\text { O } & 4.77545 & -2.82955 & 0.98499\end{array}$

$\begin{array}{llll}\text { C } & 8.52845 & 1.24154 & 2.36965\end{array}$

$\begin{array}{llll}\text { C } & 7.17607 & 1.48993 & 3.05057\end{array}$

$\begin{array}{llll}\text { C } & 8.57132 & -0.15092 & 1.72854\end{array}$

$\begin{array}{llll}\text { C } & 6.01857 & 1.28297 & 2.06407\end{array}$

$\begin{array}{llll}\text { C } & 7.41834 & -0.36118 & 0.72504\end{array}$

$\begin{array}{llll}\text { C } & 6.05328 & -0.10244 & 1.39567\end{array}$

$\begin{array}{lllll}\mathrm{O} & 4.96376 & -0.22327 & 0.49772\end{array}$

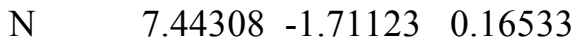




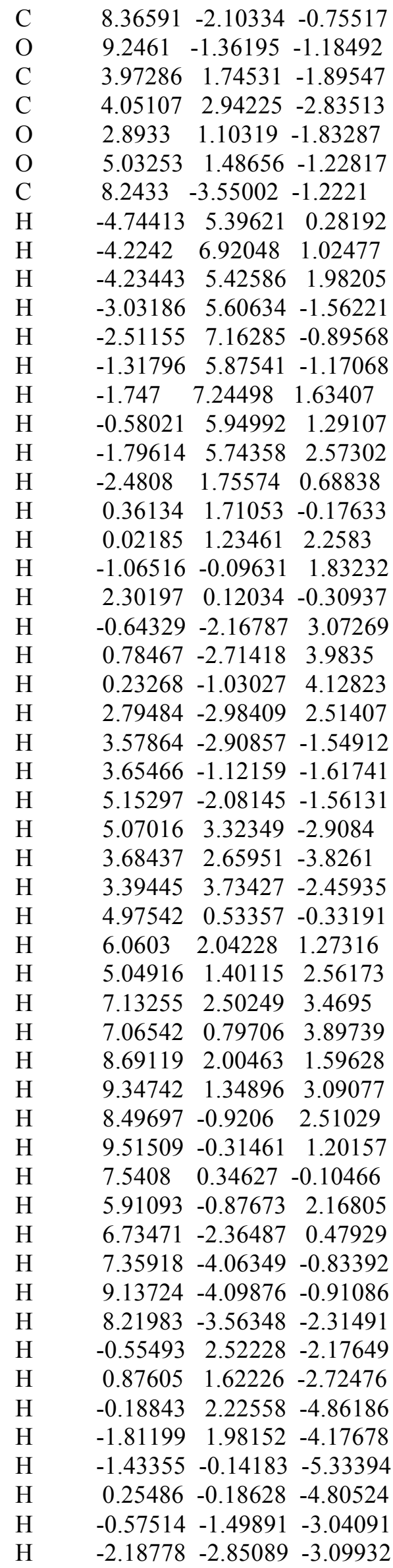

$\begin{array}{llll}\mathrm{H} & -3.5637 & -4.67033 & -2.33472 \\ \mathrm{H} & -3.70562 & -4.60814 & -4.11047 \\ \mathrm{H} & -5.15847 & -4.75814 & -3.09992 \\ \mathrm{H} & -4.69581 & -2.59919 & -5.24059 \\ \mathrm{H} & -6.16354 & -2.67288 & -4.22762 \\ \mathrm{H} & -5.2106 & -1.17943 & -4.29861 \\ \mathrm{H} & -3.29967 & -1.86811 & -0.84838 \\ \mathrm{H} & -5.97448 & -1.8292 & 0.41549 \\ \mathrm{H} & -4.45935 & 1.94111 & -1.281 \\ \mathrm{H} & -6.19993 & 2.26965 & -1.02852 \\ \mathrm{H} & -5.04256 & 2.4611 & 0.33174 \\ \mathrm{H} & -4.33481 & -3.40651 & 1.53071 \\ \mathrm{H} & -3.2806 & -2.0308 & 1.86529 \\ \mathrm{H} & -6.52287 & -3.65142 & 2.67139 \\ \mathrm{H} & -7.82493 & -3.23006 & 4.73043 \\ \mathrm{H} & -7.14957 & -1.40769 & 6.28333 \\ \mathrm{H} & -5.16398 & -0.01005 & 5.74787 \\ \mathrm{H} & -3.87755 & -0.42321 & 3.67122\end{array}$

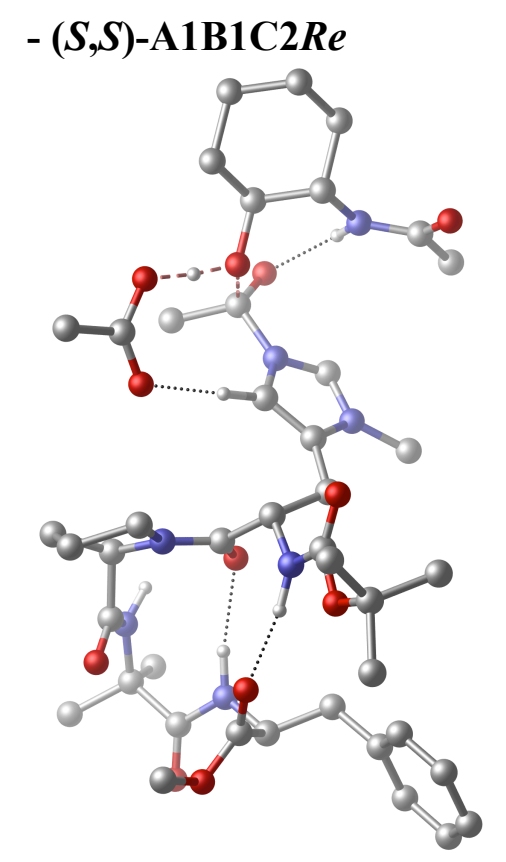

$\begin{array}{lrcl}\mathrm{N} & 1.40236 & 1.45381 & 1.03247 \\ \mathrm{C} & 0.31065 & 0.63188 & 0.53377 \\ \mathrm{C} & -0.28713 & 1.22766 & -0.7551 \\ \mathrm{C} & 0.82383 & -0.79912 & 0.2748 \\ \mathrm{C} & -1.50448 & 0.50577 & -1.24347 \\ \mathrm{O} & 1.11967 & -1.1645 & -0.87775 \\ \mathrm{~N} & -1.99439 & 0.68379 & -2.53954 \\ \mathrm{C} & -2.35017 & -0.38211 & -0.6357 \\ \mathrm{C} & -3.09001 & -0.0725 & -2.69434 \\ \mathrm{~N} & -3.32096 & -0.7253 & -1.55937 \\ \mathrm{~N} & 0.90215 & -1.62816 & 1.33025 \\ \mathrm{C} & 0.65794 & -1.28981 & 2.75011 \\ \mathrm{C} & 1.09929 & -3.07411 & 1.11904 \\ \mathrm{C} & 1.16042 & -2.53775 & 3.4832\end{array}$ 


\begin{tabular}{|c|c|c|c|}
\hline $\mathrm{C}$ & 0.80932 & -3.67424 & 2.51043 \\
\hline $\mathrm{C}$ & -1.41159 & 1.5412 & -3.56663 \\
\hline $\mathrm{C}$ & 3.81451 & -4.16461 & -1.38654 \\
\hline $\mathrm{N}$ & 2.57553 & -3.86656 & -0.65128 \\
\hline C & 4.75197 & -2.92522 & -1.47012 \\
\hline $\mathrm{O}$ & 5.95724 & +-3.06427 & -1.62539 \\
\hline $\mathrm{C}$ & 3.41554 & -4.53881 & -2.82662 \\
\hline $\mathrm{C}$ & 4.572 & -5.32445 & -0.72279 \\
\hline $\mathrm{C}$ & 1.13041 & 2.62934 & 1.67331 \\
\hline $\mathrm{O}$ & -0.00064 & $4 \quad 3.06336$ & 1.84606 \\
\hline $\mathrm{O}$ & 2.28672 & 3.21798 & 2.06035 \\
\hline C & 2.27615 & 4.49143 & 2.80037 \\
\hline $\mathrm{C}$ & 1.53266 & 4.32118 & 4.1295 \\
\hline $\mathrm{C}$ & 3.76543 & 4.74528 & 3.04432 \\
\hline $\mathrm{C}$ & 1.66776 & 5.59704 & 1.93182 \\
\hline $\mathrm{C}$ & 5.69195 & 4.11983 & -1.04652 \\
\hline $\mathrm{C}$ & 4.89005 & 2.9775 & -1.08052 \\
\hline $\mathrm{C}$ & 6.76684 & 4.25286 & -1.92576 \\
\hline $\mathrm{C}$ & 5.1495 & 1.95091 & -1.99864 \\
\hline $\mathrm{C}$ & 7.036 & $3.23528-$ & -2.84289 \\
\hline $\mathrm{C}$ & 4.29894 & 0.69732 & -2.03998 \\
\hline $\mathrm{C}$ & 6.23248 & 2.09624 & -2.87744 \\
\hline $\mathrm{C}$ & 4.91341 & -0.48122 & -1.26633 \\
\hline $\mathrm{N}$ & 4.13355 & -1.70245 & -1.45233 \\
\hline $\mathrm{C}$ & 4.95072 & -0.22811 & 0.24561 \\
\hline $\mathrm{O}$ & 4.05994 & 0.31686 & 0.87814 \\
\hline $\mathrm{O}$ & 6.03059 & -0.76304 & 0.81002 \\
\hline $\mathrm{C}$ & 6.0327 & -0.81088 & 2.25239 \\
\hline $\mathrm{O}$ & 3.51079 & -3.24193 & 1.34404 \\
\hline $\mathrm{C}$ & 2.52548 & -3.39051 & 0.62595 \\
\hline $\mathrm{C}$ & -4.51957 & $7-1.65791$ & -1.41989 \\
\hline $\mathrm{C}$ & -4.14832 & -2.96388 & -0.754 \\
\hline $\mathrm{O}$ & -5.32692 & $2-1.5797$ & -2.34401 \\
\hline $\mathrm{C}$ & -8.9553 & 1.19918 & 0.87342 \\
\hline $\mathrm{C}$ & -8.76286 & $5-0.24201$ & 1.35789 \\
\hline $\mathrm{C}$ & -8.30935 & $\begin{array}{l}5 \\
5\end{array}$ & -0.50126 \\
\hline $\mathrm{C}$ & -7.27549 & -0.61779 & 1.38243 \\
\hline $\mathrm{C}$ & -6.81219 & 1.01362 & -0.50812 \\
\hline $\mathrm{C}$ & -6.59412 & -0.42028 & 0.01689 \\
\hline $\mathrm{O}$ & -5.19606 & $6-0.7195$ & 0.10159 \\
\hline $\mathrm{N}$ & -6.27059 & 9 1.13862 & -1.86103 \\
\hline $\mathrm{C}$ & -6.05521 & 2.34298 & -2.4492 \\
\hline $\mathrm{O}$ & -6.21372 & 23.41919 & -1.87757 \\
\hline $\mathrm{C}$ & -3.48237 & $7-1.92439$ & 2.69868 \\
\hline $\mathrm{C}$ & -3.42205 & -2.35442 & 4.15914 \\
\hline $\mathrm{O}$ & -2.42853 & $3-1.84253$ & 2.02859 \\
\hline $\mathrm{O}$ & -4.66474 & $4-1.66596$ & 2.27118 \\
\hline $\mathrm{C}$ & -5.60143 & 2.29231 & -3.907 \\
\hline $\mathrm{H}$ & 4.20505 & 3.93084 & 3.62684 \\
\hline $\mathrm{H}$ & 3.9009 & 5.68031 & 3.59571 \\
\hline $\mathrm{H}$ & 4.30693 & 4.81907 & 2.09684 \\
\hline $\mathrm{H}$ & 1.96562 & 3.49499 & 4.7022 \\
\hline $\mathrm{H}$ & 1.63267 & 5.23472 & 4.7243 \\
\hline $\mathrm{H}$ & 0.47365 & 4.12244 & 3.96469 \\
\hline
\end{tabular}

\begin{tabular}{|c|c|c|c|}
\hline $\mathrm{H}$ & 1.76208 & 6.56031 & 2.44332 \\
\hline $\mathrm{H}$ & 0.61251 & 5.40447 & 1.73726 \\
\hline $\mathrm{H}$ & 2.19944 & 5.66638 & 0.97742 \\
\hline $\mathrm{H}$ & 2.36771 & 1.14316 & 0.93823 \\
\hline $\mathrm{H}$ & -0.47219 & 0.60578 & 1.29919 \\
\hline $\mathrm{H}$ & -0.53282 & 2.2728 & -0.53563 \\
\hline $\mathrm{H}$ & 0.48643 & 1.2084 & -1.52846 \\
\hline $\mathrm{H}$ & -2.35463 & -0.804 & 0.36806 \\
\hline $\mathrm{H}$ & -0.41087 & 1.1898 & -3.82813 \\
\hline $\mathrm{H}$ & -2.04648 & 1.51232 & -4.45215 \\
\hline $\mathrm{H}$ & -1.35151 & 2.5692 & -3.20362 \\
\hline $\mathrm{H}$ & -3.69302 & -0.15512 & -3.58197 \\
\hline $\mathrm{H}$ & -3.66006 & -3.59221 & -1.50785 \\
\hline $\mathrm{H}$ & -3.48417 & -2.83808 & 0.10121 \\
\hline $\mathrm{H}$ & -5.06661 & -3.45985 & -0.43752 \\
\hline $\mathrm{H}$ & -4.2011 & -3.09213 & 4.36773 \\
\hline $\mathrm{H}$ & -2.44103 & -2.76143 & 4.40908 \\
\hline $\mathrm{H}$ & -3.6216 & -1.4862 & 4.79639 \\
\hline $\mathrm{H}$ & -4.88426 & -1.17986 & 1.11671 \\
\hline $\mathrm{H}$ & -7.13825 & -1.65452 & 1.70169 \\
\hline $\mathrm{H}$ & -6.75315 & -0.00161 & 2.12416 \\
\hline $\mathrm{H}$ & -9.30722 & -0.92486 & 0.69003 \\
\hline $\mathrm{H}$ & -9.1904 & -0.37846 & 2.35813 \\
\hline $\mathrm{H}$ & -10.0206 & $\begin{array}{ll}5 & 1.4538\end{array}$ & $\begin{array}{ll}4 & 0.82639\end{array}$ \\
\hline $\mathrm{H}$ & -8.49997 & 1.89125 & 1.59532 \\
\hline $\mathrm{H}$ & -8.39799 & 2.42758 & -0.83959 \\
\hline $\mathrm{H}$ & -8.82674 & 0.76345 & -1.24011 \\
\hline $\mathrm{H}$ & -6.09617 & 0.27476 & -2.36557 \\
\hline $\mathrm{H}$ & -4.62923 & 2.78677 & -3.99745 \\
\hline $\mathrm{H}$ & -6.30836 & 2.87185 & -4.50671 \\
\hline $\mathrm{H}$ & -5.5432 & 1.28103 & -4.32078 \\
\hline $\mathrm{H}$ & 1.2045 & -0.38384 & 3.01909 \\
\hline $\mathrm{H}$ & -0.41662 & -1.15008 & 2.90493 \\
\hline $\mathrm{H}$ & 0.68525 & -2.65424 & 4.46074 \\
\hline $\mathrm{H}$ & 2.24384 & -2.49029 & 3.61837 \\
\hline $\mathrm{H}$ & 1.38167 & -4.58644 & 2.69128 \\
\hline $\mathrm{H}$ & -0.25839 & -3.90376 & 2.57215 \\
\hline $\mathrm{H}$ & 0.3708 & -3.41403 & 0.37564 \\
\hline $\mathrm{H}$ & 1.7387 & -3.73299 & -1.20195 \\
\hline $\mathrm{H}$ & 2.86545 & -3.72549 & -3.31256 \\
\hline $\mathrm{H}$ & 2.7916 & -5.43846 & -2.82776 \\
\hline $\mathrm{H}$ & 4.31433 & -4.73886 & -3.41376 \\
\hline $\mathrm{H}$ & 3.95432 & -6.22721 & -0.74775 \\
\hline $\mathrm{H}$ & 5.50513 & -5.50472 & -1.25774 \\
\hline $\mathrm{H}$ & 4.80807 & -5.08035 & 0.31339 \\
\hline $\mathrm{H}$ & 3.14104 & -1.64631 & -1.23594 \\
\hline $\mathrm{H}$ & 5.92606 & -0.69704 & -1.61043 \\
\hline $\mathrm{H}$ & 5.22029 & -1.45694 & 2.59115 \\
\hline $\mathrm{H}$ & 6.99975 & -1.23109 & 2.52467 \\
\hline $\mathrm{H}$ & 5.91271 & 0.19124 & 2.66887 \\
\hline $\mathrm{H}$ & 4.16739 & 0.36471 & -3.07503 \\
\hline $\mathrm{H}$ & 3.30259 & 0.89793 & -1.63365 \\
\hline $\mathrm{H}$ & 6.44455 & 1.31037 & -3.59881 \\
\hline $\mathrm{H}$ & 7.86873 & 3.33002 & -3.53393 \\
\hline
\end{tabular}



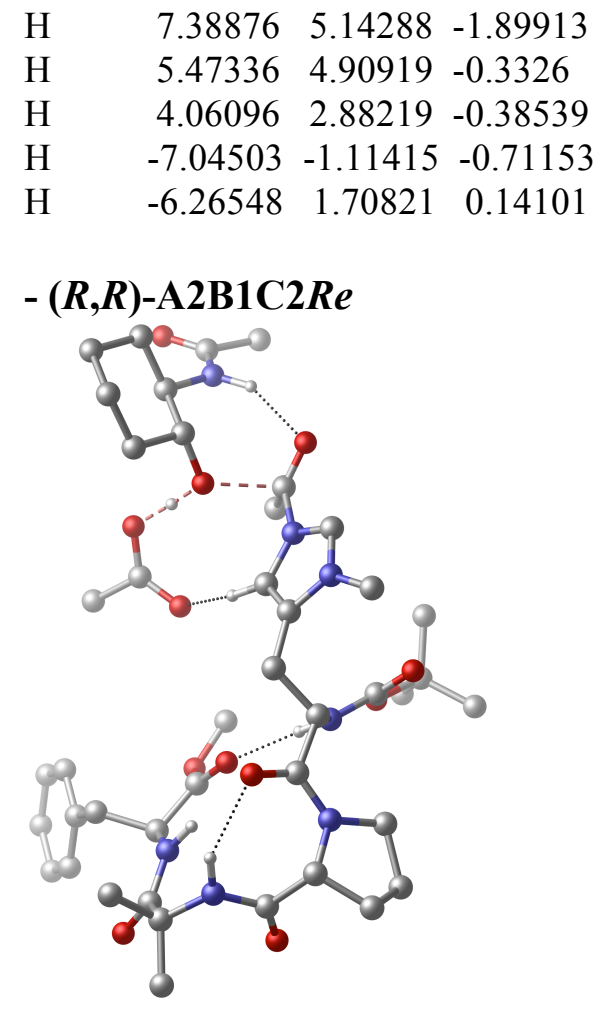

$\begin{array}{lccc}\mathrm{N} & 1.20935 & -2.27792 & 0.59181 \\ \mathrm{C} & 1.27855 & -2.61293 & -0.81388 \\ \mathrm{C} & 0.19545 & -1.87782 & -1.64418 \\ \mathrm{C} & 2.66872 & -2.33473 & -1.43047 \\ \mathrm{C} & -1.19729 & -2.05223 & -1.12895 \\ \mathrm{O} & 2.76031 & -1.66464 & -2.46879 \\ \mathrm{~N} & -1.94929 & -3.22636 & -1.26395 \\ \mathrm{C} & -2.00241 & -1.15276 & -0.48543 \\ \mathrm{C} & -3.15671 & -3.02446 & -0.72458 \\ \mathrm{~N} & -3.21016 & -1.7819 & -0.24691 \\ \mathrm{~N} & 3.75887 & -2.89068 & -0.84719 \\ \mathrm{C} & 3.81156 & -3.77085 & 0.34105 \\ \mathrm{C} & 5.06259 & -2.81836 & -1.54843 \\ \mathrm{C} & 5.30856 & -4.06732 & 0.50586 \\ \mathrm{C} & 5.86882 & -3.96437 & -0.91972 \\ \mathrm{C} & -1.47683 & -4.51059 & -1.77586 \\ \mathrm{C} & 6.00921 & 0.82995 & -2.37292 \\ \mathrm{~N} & 5.46335 & -0.53498 & -2.30939 \\ \mathrm{C} & 5.50528 & 1.71697 & -1.20088 \\ \mathrm{O} & 6.16953 & 2.66151 & -0.78226 \\ \mathrm{C} & 5.4776 & 1.46591 & -3.6738 \\ \mathrm{C} & 7.54458 & 0.81504 & -2.38228 \\ \mathrm{C} & 0.56779 & -3.10179 & 1.46977 \\ \mathrm{O} & 0.11654 & -4.20574 & 1.17381 \\ \mathrm{O} & 0.54294 & -2.53393 & 2.69414 \\ \mathrm{C} & -0.06349 & -3.22327 & 3.85066 \\ \mathrm{C} & 0.69572 & -4.52159 & 4.13973 \\ \mathrm{C} & 0.13766 & -2.21246 & 4.98145 \\ \mathrm{C} & -1.55398 & -3.46295 & 3.59389 \\ \mathrm{C} & 1.89381 & 6.33751 & 2.15219\end{array}$

\begin{tabular}{|c|c|c|c|}
\hline 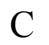 & 1.90913 & 5.31183 & 1.20516 \\
\hline $\mathrm{C}$ & 3.0913 & 6.82073 & 2.67999 \\
\hline $\mathrm{C}$ & 3.1183 & 4.75836 & 0.77108 \\
\hline $\mathrm{C}$ & 4.30437 & 6.27684 & 2.25094 \\
\hline $\mathrm{C}$ & 3.16352 & 3.65718 & -0.26426 \\
\hline C & 4.31797 & 5.25625 & 1.30142 \\
\hline $\mathrm{C}$ & 3.64873 & 2.27236 & 0.28214 \\
\hline $\mathrm{N}$ & 4.24757 & 1.4566 & -0.75564 \\
\hline $\mathrm{C}$ & 2.50465 & 1.48255 & 0.89851 \\
\hline $\mathrm{O}$ & 2.08978 & 0.42882 & 0.4321 \\
\hline $\mathrm{O}$ & 2.00472 & 2.07983 & 1.96875 \\
\hline $\mathrm{C}$ & 0.81534 & 1.4936 & 2.5602 \\
\hline $\mathrm{O}$ & 6.4816 & -1.2324 & -0.37663 \\
\hline $\mathrm{C}$ & 5.74399 & -1.44304 & -1.33397 \\
\hline $\mathrm{C}$ & -4.46676 & -1.24595 & 0.39445 \\
\hline C & -4.20296 & -0.42583 & 1.63077 \\
\hline $\mathrm{O}$ & -5.46004 & -1.93495 & 0.20473 \\
\hline $\mathrm{C}$ & -7.43544 & 1.8498 & -3.58906 \\
\hline $\mathrm{C}$ & -6.21505 & 1.10783 & -4.14999 \\
\hline $\mathrm{C}$ & -7.93362 & 1.18693 & -2.29889 \\
\hline $\mathrm{C}$ & -5.10436 & 1.00361 & -3.09614 \\
\hline $\mathrm{C}$ & -6.82514 & 1.09571 & -1.23007 \\
\hline $\mathrm{C}$ & -5.58812 & 0.35951 & -1.78523 \\
\hline $\mathrm{O}$ & -4.51166 & 0.30722 & -0.8643 \\
\hline $\mathrm{N}$ & -7.30376 & 0.41907 & $7-0.02594$ \\
\hline $\mathrm{C}$ & -8.15253 & 1.014 & 0.85641 \\
\hline $\mathrm{O}$ & -8.59078 & 2.15186 & 0.7072 \\
\hline $\mathrm{C}$ & -2.58794 & 2.65044 & 0.27945 \\
\hline $\mathrm{C}$ & -2.13557 & 4.08509 & 0.53009 \\
\hline $\mathrm{O}$ & -1.81785 & 1.70428 & 0.57674 \\
\hline $\mathrm{O}$ & -3.7461 & 2.53031 & -0.25126 \\
\hline $\mathrm{C}$ & -8.53824 & 0.16227 & 2.06128 \\
\hline $\mathrm{H}$ & 1.20075 & -2.00081 & 5.12617 \\
\hline $\mathrm{H}$ & -0.26602 & -2.61161 & 15.91631 \\
\hline $\mathrm{H}$ & -0.3757 & -1.27306 & 4.75764 \\
\hline $\mathrm{H}$ & 1.76136 & -4.31549 & 4.28043 \\
\hline $\mathrm{H}$ & 0.31388 & -4.97027 & 5.06212 \\
\hline $\mathrm{H}$ & 0.57573 & -5.23643 & 3.32537 \\
\hline $\mathrm{H}$ & -2.02398 & -3.85655 & $5 \quad 4.50052$ \\
\hline $\mathrm{H}$ & -1.7023 & -4.17641 & 2.78308 \\
\hline $\mathrm{H}$ & -2.04873 & -2.52114 & $4 \quad 3.33715$ \\
\hline $\mathrm{H}$ & 1.41743 & -1.3189 & 0.86136 \\
\hline $\mathrm{H}$ & 1.11509 & -3.69429 & -0.86758 \\
\hline $\mathrm{H}$ & 0.42393 & -0.80995 & -1.63337 \\
\hline $\mathrm{H}$ & 0.27368 & -2.19219 & -2.68975 \\
\hline $\mathrm{H}$ & -1.84103 & -0.1114 & -0.20514 \\
\hline $\mathrm{H}$ & -0.82792 & -4.97488 & $8-1.02969$ \\
\hline $\mathrm{H}$ & -2.33784 & -5.15079 & $9-1.97054$ \\
\hline $\mathrm{H}$ & -0.93465 & -4.35675 & $5-2.71044$ \\
\hline $\mathrm{H}$ & -3.96989 & -3.72976 & $6-0.67762$ \\
\hline $\mathrm{H}$ & -4.03678 & -1.12242 & $2 \quad 2.46192$ \\
\hline $\mathrm{H}$ & -3.34363 & 0.23789 & 1.52576 \\
\hline $\mathrm{H}$ & -5.09521 & 0.16364 & 1.8409 \\
\hline $\mathrm{H}$ & -2.99003 & 4.72365 & 0.76274 \\
\hline
\end{tabular}




$\begin{array}{llll}\mathrm{H} & -1.39955 & 4.1245 & 1.33593 \\ \mathrm{H} & -1.66968 & 4.47479 & -0.38259 \\ \mathrm{H} & -4.14409 & 1.3195 & -0.54939 \\ \mathrm{H} & -4.71974 & 2.00351 & -2.85832 \\ \mathrm{H} & -4.25541 & 0.42139 & -3.47238 \\ \mathrm{H} & -5.83277 & 1.61078 & -5.04641 \\ \mathrm{H} & -6.51882 & 0.09874 & -4.4643 \\ \mathrm{H} & -7.16222 & 2.89338 & -3.38099 \\ \mathrm{H} & -8.24162 & 1.88049 & -4.33154 \\ \mathrm{H} & -8.29068 & 0.17065 & -2.51952 \\ \mathrm{H} & -8.7737 & 1.73944 & -1.86979 \\ \mathrm{H} & -6.52881 & 2.11268 & -0.94387 \\ \mathrm{H} & -5.88271 & -0.68425 & -1.98451 \\ \mathrm{H} & -6.96746 & -0.52032 & 0.15361 \\ \mathrm{H} & -8.0248 & -0.80249 & 2.09979 \\ \mathrm{H} & -9.61837 & -0.00972 & 2.04114 \\ \mathrm{H} & -8.31783 & 0.72561 & 2.97207 \\ \mathrm{H} & 3.38884 & -3.26894 & 1.21098 \\ \mathrm{H} & 3.23032 & -4.68472 & 0.16043 \\ \mathrm{H} & 5.48164 & -5.04249 & 0.96837 \\ \mathrm{H} & 5.77332 & -3.29732 & 1.12574 \\ \mathrm{H} & 6.93981 & -3.75763 & -0.9331 \\ \mathrm{H} & 5.68304 & -4.88713 & -1.48033 \\ \mathrm{H} & 4.88491 & -2.96785 & -2.61727 \\ \mathrm{H} & 4.69035 & -0.77459 & -2.92126 \\ \mathrm{H} & 4.38277 & 1.47621 & -3.69477 \\ \mathrm{H} & 5.84084 & 0.90831 & -4.5428 \\ \mathrm{H} & 5.83096 & 2.49659 & -3.75079 \\ \mathrm{H} & 7.89913 & 0.27168 & -3.26331 \\ \mathrm{H} & 7.9159 & 1.84008 & -2.41353 \\ \mathrm{H} & 7.93024 & 0.33325 & -1.48427 \\ \mathrm{H} & 3.73639 & 0.64168 & -1.06715 \\ \mathrm{H} & 4.39917 & 2.46438 & 1.05605 \\ \mathrm{H} & 0.99669 & 0.44635 & 2.81239 \\ \mathrm{H} & 0.64246 & 2.07627 & 3.46413 \\ \mathrm{H} & -0.02931 & 1.58411 & 1.87004 \\ \mathrm{H} & 3.8854 & 3.92801 & -1.03912 \\ \mathrm{H} & 2.19031 & 3.5364 & -0.75203 \\ \mathrm{H} & 5.26163 & 4.83997 & 0.95649 \\ \mathrm{H} & 5.24163 & 6.65341 & 2.65095 \\ \mathrm{H} & 3.0808 & 7.61892 & 3.41666 \\ \mathrm{H} & 0.94589 & 6.75847 & 2.47551 \\ \mathrm{H} & 0.97293 & 4.93993 & 0.79742\end{array}$

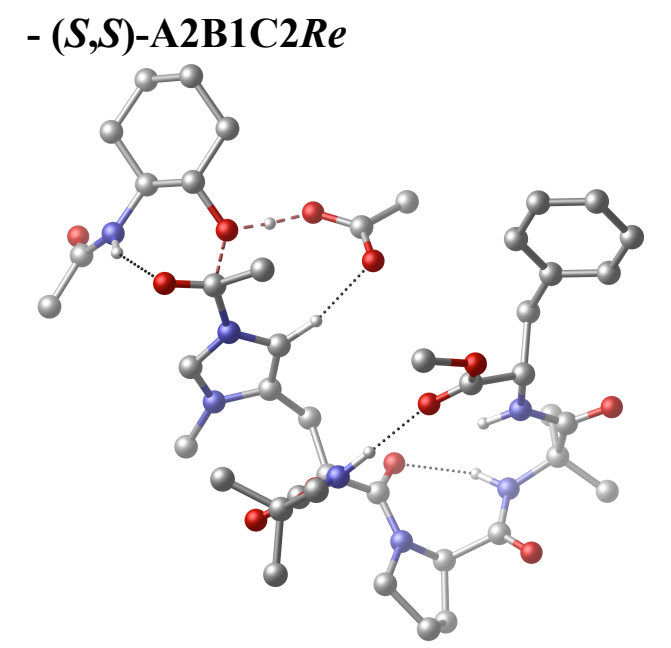

$\begin{array}{lccc}\mathrm{N} & 1.17181 & -2.37521 & 0.54368 \\ \mathrm{C} & 1.1254 & -2.57953 & -0.88966 \\ \mathrm{C} & 0.02223 & -1.72755 & -1.58168 \\ \mathrm{C} & 2.48122 & -2.27143 & -1.57166 \\ \mathrm{C} & -1.27306 & -1.66301 & -0.84185 \\ \mathrm{O} & 2.52165 & -1.49479 & -2.53633 \\ \mathrm{~N} & -2.21496 & -2.69288 & -0.76603 \\ \mathrm{C} & -1.78217 & -0.62516 & -0.11028 \\ \mathrm{C} & -3.24047 & -2.27882 & -0.01253 \\ \mathrm{~N} & -2.99963 & -1.03384 & 0.39713 \\ \mathrm{~N} & 3.59744 & -2.8941 & -1.12172 \\ \mathrm{C} & 3.71624 & -3.9035 & -0.04729 \\ \mathrm{C} & 4.86154 & -2.72624 & -1.87691 \\ \mathrm{C} & 5.22244 & -4.19377 & 0.01027 \\ \mathrm{C} & 5.70955 & -3.92287 & -1.42052 \\ \mathrm{C} & -2.09086 & -4.03099 & -1.34375 \\ \mathrm{C} & 5.76181 & 0.99656 & -2.38917 \\ \mathrm{~N} & 5.22358 & -0.37305 & -2.41632 \\ \mathrm{C} & 5.25311 & 1.81829 & -1.17194 \\ \mathrm{O} & 5.87315 & 2.79714 & -0.76484 \\ \mathrm{C} & 5.22933 & 1.7102 & -3.6492 \\ \mathrm{C} & 7.29773 & 0.99342 & -2.39642 \\ \mathrm{C} & 0.57077 & -3.25343 & 1.39613 \\ \mathrm{O} & 0.08263 & -4.32755 & 1.05368 \\ \mathrm{O} & 0.62401 & -2.775 & 2.65901 \\ \mathrm{C} & 0.08703 & -3.55036 & 3.79584 \\ \mathrm{C} & 0.85562 & -4.86764 & 3.93677 \\ \mathrm{C} & 0.36483 & -2.63036 & 4.98636 \\ \mathrm{C} & -1.4184 & -3.76877 & 3.6176 \\ \mathrm{C} & 1.04718 & 5.77726 & 2.45251 \\ \mathrm{C} & 1.23774 & 4.77966 & 1.49418 \\ \mathrm{C} & 2.1121 & 6.58877 & 2.84644 \\ \mathrm{C} & 2.49856 & 4.58749 & 0.91358 \\ \mathrm{C} & 3.37302 & 6.39861 & 2.27703 \\ \mathrm{C} & 2.73212 & 3.521 & -0.13265 \\ \mathrm{C} & 3.56333 & 5.40593 & 1.31622 \\ \mathrm{C} & 3.43209 & 2.22481 & 0.40237 \\ \mathrm{~N} & 4.04412 & 1.4595 & -0.66597 \\ & & & \\ \mathrm{n} & & & \end{array}$




\begin{tabular}{|c|c|c|c|}
\hline C & 2.41809 & 1.3373 & 1.09499 \\
\hline $\mathrm{O}$ & 1.94754 & 0.33137 & 0.58073 \\
\hline $\mathrm{O}$ & 2.06631 & 1.80505 & 2.28554 \\
\hline $\mathrm{C}$ & 0.92639 & 1.18064 & 2.91694 \\
\hline $\mathrm{O}$ & 6.31108 & -1.25592 & $2-0.59696$ \\
\hline $\mathrm{C}$ & 5.54229 & -1.37173 & $3-1.54568$ \\
\hline $\mathrm{C}$ & -4.00616 & -0.27296 & $6 \quad 1.2379$ \\
\hline $\mathrm{C}$ & -3.35977 & 0.72094 & $4 \quad 2.17398$ \\
\hline $\mathrm{O}$ & -5.02242 & -0.90457 & $\begin{array}{ll}7 & 1.50981\end{array}$ \\
\hline $\mathrm{C}$ & -8.03553 & 2.74626 & $5-1.59532$ \\
\hline $\mathrm{C}$ & -7.24303 & 3.46004 & $4-0.49256$ \\
\hline $\mathrm{C}$ & -8.01273 & 1.22753 & $3-1.38614$ \\
\hline $\mathrm{C}$ & -5.80311 & 2.93268 & -0.42049 \\
\hline $\mathrm{C}$ & -6.57462 & 0.67682 & $2-1.30835$ \\
\hline $\mathrm{C}$ & -5.75235 & 1.40863 & $3-0.22862$ \\
\hline $\mathrm{O}$ & -4.41228 & 0.92672 & $2-0.21419$ \\
\hline $\mathrm{N}$ & -6.57623 & -0.75576 & $6-1.02059$ \\
\hline $\mathrm{C}$ & -6.85342 & -1.6899 & -1.96547 \\
\hline $\mathrm{O}$ & -7.13787 & -1.41769 & $\begin{array}{ll}9 & -3.12955\end{array}$ \\
\hline $\mathrm{C}$ & -1.73068 & 2.8579 & -0.79698 \\
\hline $\mathrm{C}$ & -1.09276 & 3.9976 & -1.58304 \\
\hline $\mathrm{O}$ & -1.12262 & 2.3667 & 0.18458 \\
\hline $\mathrm{O}$ & -2.8601 & 2.45872 & -1.25788 \\
\hline $\mathrm{C}$ & -6.79682 & -3.13905 & $5-1.48663$ \\
\hline $\mathrm{H}$ & 1.43545 & -2.42649 & $9 \quad 5.07648$ \\
\hline $\mathrm{H}$ & 0.02369 & -3.10337 & $7 \quad 5.91162$ \\
\hline $\mathrm{H}$ & -0.16197 & -1.67835 & $5 \quad 4.87315$ \\
\hline $\mathrm{H}$ & 1.92912 & -4.67356 & $6 \quad 4.02464$ \\
\hline $\mathrm{H}$ & 0.53052 & -5.38587 & $7 \quad 4.84434$ \\
\hline $\mathrm{H}$ & 0.68023 & -5.51809 & $9 \quad 3.07967$ \\
\hline $\mathrm{H}$ & -1.83271 & -4.22217 & $7 \quad 4.52339$ \\
\hline $\mathrm{H}$ & -1.62109 & -4.42633 & $\begin{array}{ll}3 & 2.77222\end{array}$ \\
\hline $\mathrm{H}$ & -1.92571 & -2.81214 & $4 \quad 3.45708$ \\
\hline $\mathrm{H}$ & 1.40391 & -1.44208 & $8 \quad 0.87754$ \\
\hline $\mathrm{H}$ & 0.91848 & -3.6454 & -1.0317 \\
\hline $\mathrm{H}$ & 0.39464 & -0.70615 & $5-1.67397$ \\
\hline $\mathrm{H}$ & -0.11854 & -2.08854 & $4-2.60562$ \\
\hline $\mathrm{H}$ & -1.39808 & 0.38038 & $8 \quad 0.04812$ \\
\hline $\mathrm{H}$ & -1.34888 & -4.59782 & $2-0.77734$ \\
\hline $\mathrm{H}$ & -3.06091 & -4.52578 & $\begin{array}{ll}8 & -1.29078\end{array}$ \\
\hline $\mathrm{H}$ & -1.79549 & -3.94904 & $4-2.39141$ \\
\hline $\mathrm{H}$ & -4.12364 & -2.84215 & $\begin{array}{ll}5 & 0.23589\end{array}$ \\
\hline $\mathrm{H}$ & -2.91156 & 0.16732 & 23.00848 \\
\hline $\mathrm{H}$ & -2.60578 & 1.34151 & $1 \quad 1.687$ \\
\hline $\mathrm{H}$ & -4.15103 & 1.35503 & $3 \quad 2.57661$ \\
\hline $\mathrm{H}$ & -1.84885 & 4.56288 & $8-2.13002$ \\
\hline $\mathrm{H}$ & -0.52814 & 4.65798 & $8-0.92202$ \\
\hline $\mathrm{H}$ & -0.39179 & 3.57588 & $8-2.3129$ \\
\hline $\mathrm{H}$ & -3.62495 & 1.64218 & $8-0.6501$ \\
\hline $\mathrm{H}$ & -5.2532 & 3.40943 & 0.39888 \\
\hline $\mathrm{H}$ & -5.26625 & 3.18826 & $6-1.34093$ \\
\hline $\mathrm{H}$ & -7.74484 & 3.30276 & $\begin{array}{ll}6 & 0.47307\end{array}$ \\
\hline $\mathrm{H}$ & -7.23055 & 4.54292 & $2-0.6634$ \\
\hline $\mathrm{H}$ & -9.0708 & 3.10616 & -1.62002 \\
\hline
\end{tabular}

$\begin{array}{llll}\mathrm{H} & -7.59708 & 2.98866 & -2.57331 \\ \mathrm{H} & -8.53518 & 0.70762 & -2.19421 \\ \mathrm{H} & -8.5326 & 0.97702 & -0.45059 \\ \mathrm{H} & -6.27589 & -1.03977 & -0.09371 \\ \mathrm{H} & -6.05138 & -3.67947 & -2.07852 \\ \mathrm{H} & -7.76298 & -3.6104 & -1.68562 \\ \mathrm{H} & -6.56984 & -3.24496 & -0.42124 \\ \mathrm{H} & 3.33271 & -3.51054 & 0.89366 \\ \mathrm{H} & 3.13624 & -4.79929 & -0.30504 \\ \mathrm{H} & 5.42801 & -5.21195 & 0.3507 \\ \mathrm{H} & 5.70986 & -3.49075 & 0.68948 \\ \mathrm{H} & 6.77665 & -3.70044 & -1.46298 \\ \mathrm{H} & 5.50435 & -4.77988 & -2.07135 \\ \mathrm{H} & 4.62956 & -2.76079 & -2.94507 \\ \mathrm{H} & 4.4319 & -0.56178 & -3.02152 \\ \mathrm{H} & 4.13415 & 1.71448 & -3.67209 \\ \mathrm{H} & 5.59753 & 1.21106 & -4.5509 \\ \mathrm{H} & 5.5753 & 2.74595 & -3.65701 \\ \mathrm{H} & 7.65829 & 0.51148 & -3.31014 \\ \mathrm{H} & 7.65792 & 2.02217 & -2.36081 \\ \mathrm{H} & 7.68657 & 0.45619 & -1.53187 \\ \mathrm{H} & 3.54811 & 0.64954 & -1.01222 \\ \mathrm{H} & 4.20873 & 2.52397 & 1.11144 \\ \mathrm{H} & 1.09676 & 0.1103 & 3.05089 \\ \mathrm{H} & 0.82986 & 1.67743 & 3.88113 \\ \mathrm{H} & 0.04026 & 1.35212 & 2.30267 \\ \mathrm{H} & 3.40389 & 3.90912 & -0.9029 \\ \mathrm{H} & 1.79194 & 3.23649 & -0.61553 \\ \mathrm{H} & 4.54367 & 5.25877 & 0.86868 \\ \mathrm{H} & 4.2069 & 7.02736 & 2.57647 \\ \mathrm{H} & 1.96106 & 7.36597 & 3.59034 \\ \mathrm{H} & 0.06321 & 5.9209 & 2.89068 \\ \mathrm{H} & 0.40996 & 4.13935 & 1.19798 \\ \mathrm{H} & -6.20058 & 1.15872 & 0.74733 \\ \mathrm{H} & -6.08523 & 0.82417 & -2.27916 \\ & & & \end{array}$

\section{- $(R, R)-\mathrm{A} 3 \mathrm{~B} 1 \mathrm{C} 2 R e$}

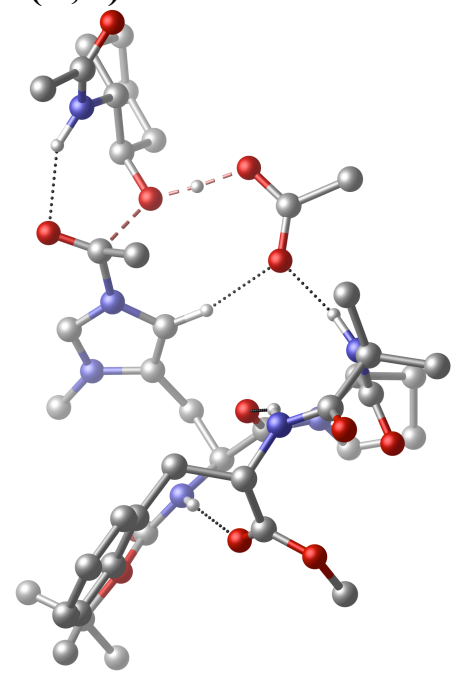




\begin{tabular}{|c|c|c|c|}
\hline N & 2.48276 & -1.60601 & -1.43601 \\
\hline $\mathrm{C}$ & 1.67103 & -1.02204 & -2.48861 \\
\hline $\mathrm{C}$ & 0.36261 & -1.82503 & -2.80279 \\
\hline $\mathrm{C}$ & 1.25884 & 0.39136 & -2.06869 \\
\hline $\mathrm{C}$ & -0.54148 & -2.0793 & -1.63563 \\
\hline $\mathrm{O}$ & 0.96027 & 0.60752 & -0.88948 \\
\hline $\mathrm{N}$ & -0.71085 & -3.34639 & -1.06588 \\
\hline $\mathrm{C}$ & -1.3415 & -1.2307 & -0.91501 \\
\hline $\mathrm{C}$ & -1.57771 & -3.24684 & -0.04959 \\
\hline $\mathrm{N}$ & -1.9631 & -1.97692 & 0.06092 \\
\hline $\mathrm{N}$ & 1.09298 & 1.31552 & -3.04244 \\
\hline $\mathrm{C}$ & 1.6798 & 1.31205 & -4.39585 \\
\hline $\mathrm{C}$ & 0.34839 & 2.56104 & -2.75108 \\
\hline $\mathrm{C}$ & 1.66406 & 2.79693 & -4.77657 \\
\hline C & 0.38756 & 3.31543 & -4.09909 \\
\hline $\mathrm{C}$ & -0.12468 & -4.60499 & -1.53241 \\
\hline $\mathrm{C}$ & 0.53492 & 4.49288 & 0.5578 \\
\hline $\mathrm{N}$ & 0.16338 & 3.69793 & -0.62196 \\
\hline $\mathrm{C}$ & 1.6551 & 3.81795 & 1.40026 \\
\hline $\mathrm{O}$ & 2.31174 & 4.47505 & 2.19888 \\
\hline $\mathrm{C}$ & -0.70869 & 4.58566 & 1.46336 \\
\hline $\mathrm{C}$ & 1.00071 & 5.89988 & 0.14881 \\
\hline $\mathrm{C}$ & 3.13927 & -2.77801 & -1.65617 \\
\hline $\mathrm{O}$ & 2.89735 & -3.52994 & -2.60146 \\
\hline $\mathrm{O}$ & 4.04695 & -3.00417 & -0.68348 \\
\hline $\mathrm{C}$ & 4.94491 & -4.17383 & -0.73131 \\
\hline $\mathrm{C}$ & 5.81732 & -4.10777 & -1.98863 \\
\hline $\mathrm{C}$ & 5.7938 & -3.99222 & 0.52846 \\
\hline $\mathrm{C}$ & 4.13438 & -5.47155 & -0.65292 \\
\hline $\mathrm{C}$ & 5.3801 & -1.69861 & 3.73438 \\
\hline $\mathrm{C}$ & 4.37676 & -1.15714 & 2.92854 \\
\hline C & 5.53842 & -1.26475 & 5.05107 \\
\hline $\mathrm{C}$ & 3.51468 & -0.17123 & 3.4276 \\
\hline C & 4.68544 & -0.28342 & 5.55887 \\
\hline $\mathrm{C}$ & 2.43804 & 0.45096 & 2.56163 \\
\hline C & 3.68428 & 0.25664 & 4.75216 \\
\hline $\mathrm{C}$ & 2.89325 & 1.74938 & 1.87135 \\
\hline $\mathrm{N}$ & 1.77652 & 2.46301 & 1.26274 \\
\hline $\mathrm{C}$ & 3.90356 & 1.50027 & 0.7461 \\
\hline $\mathrm{O}$ & 3.94995 & 0.50261 & 0.04358 \\
\hline $\mathrm{O}$ & 4.684 & 2.56725 & 0.56162 \\
\hline $\mathrm{C}$ & 5.46623 & 2.58773 & -0.64673 \\
\hline $\mathrm{O}$ & 2.19884 & 3.71186 & -1.69739 \\
\hline $\mathrm{C}$ & 1.01586 & 3.37533 & -1.62663 \\
\hline $\mathrm{C}$ & -2.94433 & -1.54254 & $4 \quad 1.14363$ \\
\hline $\mathrm{C}$ & -2.518 & -0.26234 & 1.82217 \\
\hline $\mathrm{O}$ & -3.485 & -2.4859 & 1.71575 \\
\hline $\mathrm{C}$ & -8.27987 & -1.5022 & -1.00206 \\
\hline $\mathrm{C}$ & -7.20706 & -2.05924 & $4-1.9474$ \\
\hline $\mathrm{C}$ & -7.82928 & -1.60804 & $4 \quad 0.45984$ \\
\hline $\mathrm{C}$ & -5.85745 & -1.36509 & -1.71664 \\
\hline $\mathrm{C}$ & -6.48048 & -0.89941 & $1 \quad 0.70192$ \\
\hline $\mathrm{C}$ & -5.39867 & -1.44741 & -0.25149 \\
\hline $\mathrm{O}$ & -4.14972 & -0.7787 & -0.11465 \\
\hline
\end{tabular}

\begin{tabular}{|c|c|c|c|}
\hline $\mathrm{N}$ & -6.04339 & -1.0508 & 2.08713 \\
\hline $\mathrm{C}$ & -6.61203 & -0.35162 & 3.10726 \\
\hline $\mathrm{O}$ & -7.53476 & 0.44336 & 2.94505 \\
\hline $\mathrm{C}$ & -3.43391 & 2.33883 & -0.79067 \\
\hline $\mathrm{C}$ & -3.79139 & 3.77888 & -1.1321 \\
\hline $\mathrm{O}$ & -2.22852 & 1.98251 & -0.73677 \\
\hline $\mathrm{O}$ & -4.43572 & 1.57975 & -0.5551 \\
\hline $\mathrm{C}$ & -6.02767 & -0.62649 & 4.48828 \\
\hline $\mathrm{H}$ & 6.32626 & -3.0377 & 0.4987 \\
\hline $\mathrm{H}$ & 6.52875 & -4.79906 & 0.60337 \\
\hline $\mathrm{H}$ & 5.16587 & -4.01103 & 1.42357 \\
\hline $\mathrm{H}$ & 6.35492 & -3.15546 & -2.0271 \\
\hline $\mathrm{H}$ & 6.55695 & -4.91427 & -1.96337 \\
\hline $\mathrm{H}$ & 5.21493 & -4.2134 & -2.8913 \\
\hline $\mathrm{H}$ & 4.81486 & -6.32042 & -0.53168 \\
\hline $\mathrm{H}$ & 3.54516 & -5.62251 & -1.55742 \\
\hline $\mathrm{H}$ & 3.46669 & -5.44918 & 0.21438 \\
\hline $\mathrm{H}$ & 2.8517 & -0.97159 & -0.72738 \\
\hline $\mathrm{H}$ & 2.2329 & -1.00933 & -3.42864 \\
\hline $\mathrm{H}$ & -0.18701 & -1.26814 & -3.56942 \\
\hline $\mathrm{H}$ & 0.67463 & -2.76803 & -3.25227 \\
\hline $\mathrm{H}$ & -1.52484 & -0.16813 & -1.0082 \\
\hline $\mathrm{H}$ & 0.92852 & -4.46043 & -1.77573 \\
\hline $\mathrm{H}$ & -0.21969 & -5.34642 & -0.7386 \\
\hline $\mathrm{H}$ & -0.65577 & -4.95979 & -2.41991 \\
\hline $\mathrm{H}$ & -1.92916 & -4.04401 & 0.58426 \\
\hline $\mathrm{H}$ & -1.69084 & -0.49975 & 2.50071 \\
\hline $\mathrm{H}$ & -2.20459 & 0.50989 & 1.11863 \\
\hline $\mathrm{H}$ & -3.36185 & 0.10172 & 2.40887 \\
\hline $\mathrm{H}$ & -4.20687 & 4.2651 & -0.24344 \\
\hline $\mathrm{H}$ & -2.91809 & 4.33787 & -1.47218 \\
\hline $\mathrm{H}$ & -4.57074 & 3.79647 & -1.89842 \\
\hline $\mathrm{H}$ & -4.22594 & 0.35516 & -0.28621 \\
\hline $\mathrm{H}$ & -5.93245 & -0.30515 & -1.99073 \\
\hline $\mathrm{H}$ & -5.07558 & -1.80068 & -2.34947 \\
\hline $\mathrm{H}$ & -7.51242 & -1.94193 & -2.99411 \\
\hline $\mathrm{H}$ & -7.09784 & -3.13944 & -1.77324 \\
\hline $\mathrm{H}$ & -8.47152 & -0.44906 & -1.24969 \\
\hline $\mathrm{H}$ & -9.22862 & -2.03315 & -1.14343 \\
\hline $\mathrm{H}$ & -7.72359 & -2.66615 & 0.73908 \\
\hline $\mathrm{H}$ & -8.56828 & -1.16741 & 1.13429 \\
\hline $\mathrm{H}$ & -6.60913 & 0.17297 & 0.50903 \\
\hline $\mathrm{H}$ & -5.21663 & -2.50028 & 0.01466 \\
\hline $\mathrm{H}$ & -5.27325 & -1.68549 & 2.27041 \\
\hline $\mathrm{H}$ & -5.16598 & -1.29958 & 4.47449 \\
\hline $\mathrm{H}$ & -6.80713 & -1.05989 & 5.12183 \\
\hline $\mathrm{H}$ & -5.73601 & 0.32522 & 4.94046 \\
\hline $\mathrm{H}$ & 2.68901 & 0.89365 & -4.38314 \\
\hline $\mathrm{H}$ & 1.06767 & 0.71829 & -5.08927 \\
\hline $\mathrm{H}$ & 1.67312 & 2.94641 & -5.85944 \\
\hline $\mathrm{H}$ & 2.53284 & 3.29403 & -4.33836 \\
\hline $\mathrm{H}$ & 0.39218 & 4.39858 & -3.9599 \\
\hline $\mathrm{H}$ & -0.49734 & 3.05557 & -4.69058 \\
\hline $\mathrm{H}$ & -0.67051 & 2.30085 & -2.4536 \\
\hline
\end{tabular}




$\begin{array}{lllc}\mathrm{H} & -0.73583 & 3.2043 & -0.6058 \\ \mathrm{H} & -1.07962 & 3.59146 & 1.7306 \\ \mathrm{H} & -1.51044 & 5.1295 & 0.95435 \\ \mathrm{H} & -0.45021 & 5.11968 & 2.38007 \\ \mathrm{H} & 0.18063 & 6.42652 & -0.34904 \\ \mathrm{H} & 1.3042 & 6.45482 & 1.03736 \\ \mathrm{H} & 1.8508 & 5.83705 & -0.53142 \\ \mathrm{H} & 1.2895 & 1.99114 & 0.50574 \\ \mathrm{H} & 3.33825 & 2.43919 & 2.59157 \\ \mathrm{H} & 4.79792 & 2.7065 & -1.50291 \\ \mathrm{H} & 6.12047 & 3.45372 & -0.55439 \\ \mathrm{H} & 6.04865 & 1.66955 & -0.74614 \\ \mathrm{H} & 1.57038 & 0.71026 & 3.17683 \\ \mathrm{H} & 2.09707 & -0.25325 & 1.79647 \\ \mathrm{H} & 3.0217 & 1.01921 & 5.15478 \\ \mathrm{H} & 4.7974 & 0.06001 & 6.58337 \\ \mathrm{H} & 6.31852 & -1.68832 & 5.67711 \\ \mathrm{H} & 6.04064 & -2.4613 & 3.33139 \\ \mathrm{H} & 4.26265 & -1.4953 & 1.90301\end{array}$

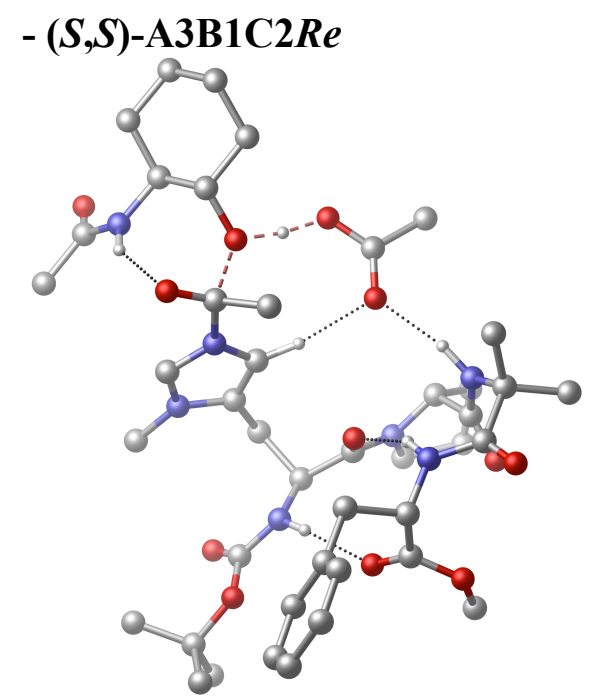

$\begin{array}{lccc}\mathrm{N} & -1.90026 & 1.75976 & -1.48661 \\ \mathrm{C} & -1.13716 & 0.98096 & -2.44493 \\ \mathrm{C} & 0.35168 & 1.44603 & -2.60671 \\ \mathrm{C} & -1.11668 & -0.47962 & -1.9911 \\ \mathrm{C} & 1.1499 & 1.54147 & -1.34251 \\ \mathrm{O} & -0.98829 & -0.7378 & -0.78997 \\ \mathrm{~N} & 1.5184 & 2.76356 & -0.77209 \\ \mathrm{C} & 1.66094 & 0.57094 & -0.51842 \\ \mathrm{C} & 2.21641 & 2.52054 & 0.34423 \\ \mathrm{~N} & 2.3132 & 1.20438 & 0.51628 \\ \mathrm{~N} & -1.08758 & -1.43781 & -2.94596 \\ \mathrm{C} & -1.52801 & -1.32834 & -4.34945 \\ \mathrm{C} & -0.67253 & -2.8113 & -2.58748 \\ \mathrm{C} & -1.81561 & -2.78592 & -4.72775 \\ \mathrm{C} & -0.75746 & -3.56546 & -3.93362 \\ \mathrm{C} & 1.28033 & 4.10013 & -1.32188 \\ \mathrm{C} & -1.56698 & -4.56623 & 0.70628\end{array}$

\begin{tabular}{|c|c|c|}
\hline & $-0.92904-3.90511$ & -0.44185 \\
\hline & $-2.58261-3.64218$ & 1.43838 \\
\hline & $-3.43202-4.12151$ & 2.17989 \\
\hline C & $-0.45543-4.90093$ & 1.72007 \\
\hline $\mathrm{c}$ & $-2.29069-5.85002$ & 0.26835 \\
\hline $\mathrm{C}$ & $-2.2576 \quad 3.03873$ & -1.78554 \\
\hline $\mathrm{C}$ & -1.770813 .683 & -2.71531 \\
\hline $\mathrm{C}$ & -3.17539 & -0.9065 \\
\hline $\mathrm{C}$ & -3.77571 & -1.05206 \\
\hline $\mathrm{C}$ & -4.53045 & -2.38212 \\
\hline $\mathrm{C}$ & -4.74686 & 0.12864 \\
\hline $\mathrm{C}$ & -2.70061 & -0.9207 \\
\hline $\mathrm{C}$ & -5.20849 & 3.34691 \\
\hline $\mathrm{C}$ & $-4.2736 \quad 1.84942$ & 2.65657 \\
\hline $\mathrm{C}$ & $\begin{array}{ll}-5.60175 & 2.26901\end{array}$ & 4.63799 \\
\hline $\mathrm{C}$ & -3.71585 & 3.24793 \\
\hline $\mathrm{C}$ & -5.05352 & 5.23658 \\
\hline$c$ & $-2.721 \quad-0.16152$ & 2.506 \\
\hline$c$ & -4.119820 .3619 & 4.54541 \\
\hline $\mathrm{C}$ & $-3.38219-1.34314$ & 1.77358 \\
\hline 1 & $-2.39744-2.29773$ & 1.2771 \\
\hline $\mathrm{C}$ & $-4.19811-0.90024$ & 0.55386 \\
\hline $\mathrm{C}$ & $\begin{array}{ll}-3.94691 & 0.06262\end{array}$ & -0.15345 \\
\hline $\mathrm{C}$ & $-5.18245-1.7651$ & 0.29798 \\
\hline $\mathrm{C}$ & $-5.83124-1.63561$ & -0.98062 \\
\hline $\mathrm{C}$ & $-2.81276-3.52013$ & -1.70816 \\
\hline C & $-1.59803-3.43569$ & -1.52617 \\
\hline C & $3.04675 \quad 0.63585$ & 1.74438 \\
\hline $\mathrm{C}$ & $2.27506-0.50507$ & 2.37172 \\
\hline $\mathrm{C}$ & $3.62161 \quad 1.50276$ & 2.40615 \\
\hline $\mathrm{C}$ & $8.55503-0.59486$ & 1.12921 \\
\hline C & $\begin{array}{lll}7.68659 & -1.53877\end{array}$ & 1.97021 \\
\hline C & $7.92042 \quad 0.79799$ & 1.05045 \\
\hline C & $6.26095-1.61607$ & 1.40669 \\
\hline C & $6.47662 \quad 0.75392$ & 0.50635 \\
\hline C & $\begin{array}{lll}5.60619 & -0.22833\end{array}$ & 1.314 \\
\hline $\mathrm{C}$ & $4.28695-0.30415$ & 0.76262 \\
\hline 1 & 2.08232 & 0.54813 \\
\hline $\mathrm{c}$ & $6.18256 \quad 3.05235$ & -0.34807 \\
\hline C & $6.96978 \quad 2.88969$ & -1.278 \\
\hline C & $2.89702-3.06626$ & -0.53769 \\
\hline $\mathrm{C}$ & $3.08246-4.46463$ & -1.10774 \\
\hline $\mathrm{C}$ & $1.75102-2.63451$ & -0.2724 \\
\hline C & $3.98882-2.41192$ & -0.37709 \\
\hline $\mathrm{C}$ & $5.48557 \quad 4.39387$ & -0.13492 \\
\hline 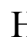 & $-5.47868 \quad 4.08242$ & 0.06531 \\
\hline $\mathrm{H}$ & $-5.2822 \quad 5.84643$ & 0.12488 \\
\hline 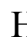 & $-4.20914 \quad 4.80009$ & 1.07645 \\
\hline F & $-5.27023 \quad 4.11694$ & -2.45103 \\
\hline 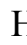 & $-5.06074 \quad 5.87607$ & -2.44109 \\
\hline F & $-3.84555 \quad 4.8471$ & -3.22735 \\
\hline P & $-3.17825 \quad 6.90073$ & -0.87429 \\
\hline I & -2.01785 & -1.77012 \\
\hline & $-2.13149 \quad 5.77538$ & 0.00374 \\
\hline
\end{tabular}




\begin{tabular}{|c|c|c|c|}
\hline $\mathrm{H}$ & -2.46316 & 1.24504 & -0.80991 \\
\hline $\mathrm{H}$ & -1.58001 & 1.08144 & -3.44141 \\
\hline $\mathrm{H}$ & 0.84171 & 0.7455 & -3.29137 \\
\hline $\mathrm{H}$ & 0.32402 & 2.41439 & -3.10714 \\
\hline $\mathrm{H}$ & 1.59928 & -0.50916 & -0.56833 \\
\hline $\mathrm{H}$ & 0.26287 & 4.17223 & -1.70748 \\
\hline $\mathrm{H}$ & 1.42271 & 4.83202 & -0.52632 \\
\hline $\mathrm{H}$ & 1.98969 & 4.30483 & -2.12838 \\
\hline $\mathrm{H}$ & 2.63392 & 3.25201 & 1.01442 \\
\hline $\mathrm{H}$ & 1.40731 & -0.08675 & 2.89384 \\
\hline $\mathrm{H}$ & 1.94155 & -1.24717 & 1.64539 \\
\hline $\mathrm{H}$ & 2.92398 & -0.97794 & 3.11088 \\
\hline $\mathrm{H}$ & 3.71871 & -5.05033 & -0.43765 \\
\hline $\mathrm{H}$ & 2.12499 & -4.97087 & -1.23843 \\
\hline $\mathrm{H}$ & 3.60374 & -4.40307 & -2.06776 \\
\hline $\mathrm{H}$ & 4.0416 & -1.33524 & 0.21167 \\
\hline $\mathrm{H}$ & 5.63013 & -2.26298 & 2.02648 \\
\hline $\mathrm{H}$ & 6.28364 & -2.07072 & 0.41049 \\
\hline $\mathrm{H}$ & 7.65648 & -1.17618 & 3.00747 \\
\hline $\mathrm{H}$ & 8.12453 & -2.54331 & 2.00332 \\
\hline $\mathrm{H}$ & 9.56543 & -0.52341 & 1.54865 \\
\hline $\mathrm{H}$ & 8.66551 & -1.00746 & 0.11678 \\
\hline $\mathrm{H}$ & 8.50472 & 1.46586 & 0.4112 \\
\hline $\mathrm{H}$ & 7.89733 & 1.24746 & 2.05311 \\
\hline $\mathrm{H}$ & 5.18003 & 2.24533 & 1.27554 \\
\hline $\mathrm{H}$ & 4.85194 & 4.6093 & -1.00114 \\
\hline $\mathrm{H}$ & 6.24717 & 5.17673 & -0.09391 \\
\hline $\mathrm{H}$ & 4.88493 & 4.44058 & 0.77831 \\
\hline $\mathrm{H}$ & -2.41191 & -0.6916 & -4.43331 \\
\hline $\mathrm{H}$ & -0.73461 & -0.90392 & -4.98041 \\
\hline $\mathrm{H}$ & -1.75925 & -2.95249 & -5.80662 \\
\hline $\mathrm{H}$ & -2.81078 & -3.06456 & $5-4.37279$ \\
\hline $\mathrm{H}$ & -1.02022 & -4.61659 & -3.79686 \\
\hline $\mathrm{H}$ & 0.2136 & -3.52318 & -4.43913 \\
\hline $\mathrm{H}$ & 0.3475 & -2.77367 & -2.19815 \\
\hline $\mathrm{H}$ & 0.04087 & -3.59596 & -0.33276 \\
\hline $\mathrm{H}$ & 0.10182 & -4.0042 & 2.00813 \\
\hline $\mathrm{H}$ & 0.24677 & -5.62238 & 1.2902 \\
\hline $\mathrm{H}$ & -0.90205 & -5.33736 & 2.61586 \\
\hline $\mathrm{H}$ & -1.56603 & -6.55157 & -0.15645 \\
\hline $\mathrm{H}$ & -2.77624 & -6.30604 & 1.13202 \\
\hline $\mathrm{H}$ & -3.05032 & -5.62283 & -0.48031 \\
\hline $\mathrm{H}$ & -1.75091 & -1.96073 & 0.56881 \\
\hline $\mathrm{H}$ & -4.03627 & -1.902 & 2.44606 \\
\hline $\mathrm{H}$ & -5.12835 & -1.91705 & -1.76834 \\
\hline $\mathrm{H}$ & -6.66822 & -2.33215 & -0.95353 \\
\hline $\mathrm{H}$ & -6.18145 & -0.61305 & -1.13512 \\
\hline $\mathrm{H}$ & -2.00289 & -0.59093 & 3.21212 \\
\hline $\mathrm{H}$ & -2.15217 & 0.42846 & 1.78069 \\
\hline $\mathrm{H}$ & -3.69447 & -0.51988 & 5.01889 \\
\hline $\mathrm{H}$ & -5.35007 & 0.84961 & 6.24238 \\
\hline $\mathrm{H}$ & -6.32799 & 2.87304 & 5.17427 \\
\hline $\mathrm{H}$ & -5.63104 & 3.50512 & 2.87368 \\
\hline $\mathrm{H}$ & -3.9774 & 2.12658 & 1.64932 \\
\hline
\end{tabular}

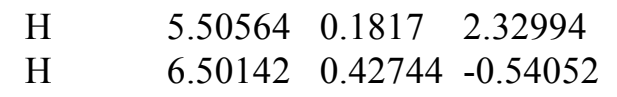

\section{- $(R, R)-\mathrm{A} 1 \mathrm{~B} 2 \mathrm{C} 2 R e$}

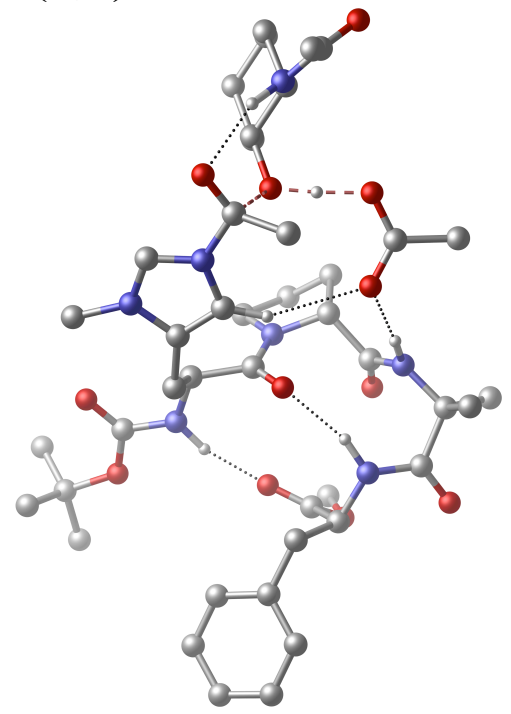

$\begin{array}{llll}\mathrm{N} & -2.49981 & -1.96067 & 0.57934\end{array}$

C $\quad-1.11036-1.75036 \quad 0.22266$

$\begin{array}{lllll}\text { C } & & -0.8368 & -2.08584 & -1.26655\end{array}$

$\begin{array}{llll}\text { C } & -0.69949 & -0.28154 & 0.45886\end{array}$

C $\quad 0.62436 \quad-2.17351-1.56946$

$\begin{array}{lllll}\mathrm{O} & -0.72208 & 0.51097 & -0.49455\end{array}$

$\mathrm{N} \quad 1.29656 \quad-3.39536-1.6738$

$\begin{array}{lllll}\mathrm{C} & 1.57516 & -1.19994 & -1.73473\end{array}$

$\begin{array}{llll}\mathrm{C} & 2.59059 & -3.15586 & -1.91846\end{array}$

$\mathrm{N} \quad 2.78037 \quad-1.83663-1.96187$

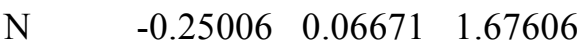

$\begin{array}{llll}\mathrm{C} & -0.33281 & -0.73048 & 2.91136\end{array}$

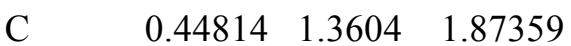

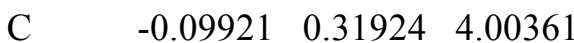

$\begin{array}{llll}\text { C } & 0.89932 & 1.28704 & 3.34877\end{array}$

$\begin{array}{llll}\text { C } & 0.68865 & -4.72456 & -1.56328\end{array}$

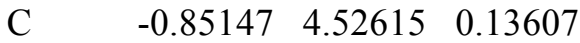

$\begin{array}{llll}\mathrm{N} & -0.09352 & 3.3497 & 0.5881\end{array}$

C $\quad-2.32519 \quad 4.16526-0.21116$

$\begin{array}{llll}\mathrm{O} & -3.2078 & 5.01147 & -0.15892\end{array}$

$\begin{array}{llll}\text { C } & -0.19227 & 5.03834 & -1.15908\end{array}$

$\begin{array}{llll}\text { C } & -0.85063 & 5.62568 & 1.21018\end{array}$

$\begin{array}{llll}\text { C } & -2.96914 & -3.23604 & 0.68646\end{array}$

$\begin{array}{lllll}\mathrm{O} & -2.26325 & -4.23357 & 0.54495\end{array}$

$\begin{array}{lllll}\text { O } & -4.28938 & -3.22484 & 0.96026\end{array}$

$\begin{array}{llll}\text { C } & -5.0488 & -4.47494 & 1.14264\end{array}$

$\begin{array}{llll}\text { C } & -4.49315 & -5.25473 & 2.33837\end{array}$

$\begin{array}{llll}\text { C } & -6.46046 & -3.96292 & 1.43648\end{array}$

$\begin{array}{llll}\text { C } & -5.02474 & -5.29564 & -0.15065\end{array}$

$\begin{array}{lllll}\text { C } & -7.23069 & -0.69708 & -1.99649\end{array}$

$\begin{array}{llll}\text { C } & -5.91365 & -0.30019 & -1.7582\end{array}$

$\begin{array}{lllll}\text { C } & & -8.10207 & 0.13809 & -2.69607\end{array}$ 


\begin{tabular}{|c|c|c|}
\hline $\mathrm{C}$ & -5.44444 & $1-2.21875$ \\
\hline $\mathrm{C}$ & -7.64736 & $8-3.15717$ \\
\hline $\mathrm{C}$ & -4.02129 & -1.96079 \\
\hline $\mathrm{C}$ & $-6.33066 \quad 1.7677$ & -2.92009 \\
\hline $\mathrm{C}$ & $-3.893 \quad 2.36031$ & -0.77524 \\
\hline $\mathrm{N}$ & $\begin{array}{lll}-2.53769 & 2.8897\end{array}$ & -0.66625 \\
\hline $\mathrm{C}$ & -4.20544 & $\begin{array}{ll}6 & 0.56534\end{array}$ \\
\hline $\mathrm{O}$ & -3.92697 & 0.84717 \\
\hline $\mathrm{O}$ & -4.74623 & 1.42974 \\
\hline $\mathrm{C}$ & -4.82365 & 2.79972 \\
\hline $\mathrm{O}$ & $\begin{array}{ll}-1.49458 & 2.74117\end{array}$ & 2.30093 \\
\hline $\mathrm{C}$ & $\begin{array}{ll}-0.48848 & 2.55492\end{array}$ & 1.61112 \\
\hline $\mathrm{C}$ & $4.15312-1.26367$ & $7-2.2175$ \\
\hline $\mathrm{C}$ & $4.1283 \quad 0.07064$ & -2.91552 \\
\hline $\mathrm{O}$ & $5.02846-2.11023$ & $3-2.33684$ \\
\hline $\mathrm{C}$ & $6.95852-1.19266$ & $\begin{array}{ll}6 \quad 2.90072\end{array}$ \\
\hline $\mathrm{C}$ & $5.5553-1.81175$ & 2.95193 \\
\hline $\mathrm{C}$ & $7.52213-1.2294$ & 1.47537 \\
\hline $\mathrm{C}$ & $4.61995-1.13204$ & $4 \quad 1.94244$ \\
\hline $\mathrm{C}$ & $6.59121-0.52608$ & 0.46554 \\
\hline $\mathrm{C}$ & $5.17873-1.14714$ & $\begin{array}{ll}4 & 0.50928\end{array}$ \\
\hline $\mathrm{O}$ & $4.24988-0.51155$ & $5-0.35736$ \\
\hline $\mathrm{N}$ & $\begin{array}{lll}7.12851 & -0.59748\end{array}$ & $8-0.89067$ \\
\hline $\mathrm{C}$ & 8.189150 .158 & -1.2892 \\
\hline $\mathrm{O}$ & $8.7805 \quad 0.93326$ & -0.54252 \\
\hline $\mathrm{C}$ & $3.42914 \quad 2.63468$ & -0.33066 \\
\hline $\mathrm{C}$ & $3.61787 \quad 4.12394$ & $4-0.06138$ \\
\hline $\mathrm{O}$ & $2.29656 \quad 2.21768$ & $8-0.6907$ \\
\hline $\mathrm{O}$ & $4.46702 \quad 1.90583$ & $3-0.17726$ \\
\hline $\mathrm{C}$ & $\begin{array}{lll}8.60794 & -0.02564\end{array}$ & $4-2.74361$ \\
\hline $\mathrm{H}$ & $-6.46555-3.33951$ & $1 \quad 2.33472$ \\
\hline $\mathrm{H}$ & $-7.13949-4.80602$ & 1.5936 \\
\hline $\mathrm{H}$ & $-6.83665-3.36446$ & 0.60196 \\
\hline $\mathrm{H}$ & $-4.48988-4.62512$ & 3.23349 \\
\hline $\mathrm{H}$ & $-5.12954-6.12283$ & 2.5376 \\
\hline $\mathrm{H}$ & $\begin{array}{ll}-3.47767 & -5.60042\end{array}$ & $2 \quad 2.14377$ \\
\hline $\mathrm{H}$ & $-5.68495-6.16244$ & $4-0.04637$ \\
\hline $\mathrm{H}$ & $-4.01687-5.64665$ & $5-0.3735$ \\
\hline $\mathrm{H}$ & $-5.38673-4.6934$ & -0.98982 \\
\hline $\mathrm{H}$ & $-3.1374-1.16701$ & 0.63922 \\
\hline $\mathrm{H}$ & $\begin{array}{ll}-0.49869 & -2.40972\end{array}$ & $2 \quad 0.84739$ \\
\hline $\mathrm{H}$ & $-1.32923-3.03168$ & $8-1.49494$ \\
\hline $\mathrm{H}$ & $-1.28461-1.30024$ & $4-1.87655$ \\
\hline $\mathrm{H}$ & $1.4944 \quad-0.12612$ & -1.64039 \\
\hline $\mathrm{H}$ & $0.15463-4.97304$ & $4-2.48398$ \\
\hline $\mathrm{H}$ & $1.47895-5.4566$ & -1.39596 \\
\hline $\mathrm{H}$ & $-0.01238-4.74149$ & $9-0.72641$ \\
\hline $\mathrm{H}$ & $\begin{array}{lll}3.37171 & -3.88348\end{array}$ & $8-2.06369$ \\
\hline $\mathrm{H}$ & $3.92387-0.10709$ & 9 -3.97793 \\
\hline $\mathrm{H}$ & $\begin{array}{ll}3.3863 & 0.75605\end{array}$ & -2.50556 \\
\hline $\mathrm{H}$ & 5.115720 .5203 & -2.8146 \\
\hline $\mathrm{H}$ & 3.44544 & $6-0.9861$ \\
\hline $\mathrm{H}$ & 2.87599 & $7 \quad 0.66736$ \\
\hline $\mathrm{H}$ & $4.62228 \quad 4.33455$ & $5 \quad 0.3069$ \\
\hline
\end{tabular}

\begin{tabular}{llll} 
H & 4.31222 & 0.59639 & -0.29798 \\
$\mathrm{H}$ & 4.46343 & -0.08315 & 2.22806 \\
$\mathrm{H}$ & 3.63238 & -1.60842 & 1.93264 \\
$\mathrm{H}$ & 5.1339 & -1.73759 & 3.9618 \\
$\mathrm{H}$ & 5.6244 & -2.88473 & 2.72159 \\
$\mathrm{H}$ & 6.9091 & -0.15113 & 3.24677 \\
$\mathrm{H}$ & 7.63384 & -1.71692 & 3.58699 \\
$\mathrm{H}$ & 7.65916 & -2.27321 & 1.15783 \\
$\mathrm{H}$ & 8.50023 & -0.74411 & 1.42585 \\
$\mathrm{H}$ & 6.5178 & 0.53469 & 0.73408 \\
$\mathrm{H}$ & 5.26283 & -2.19197 & 0.16632 \\
$\mathrm{H}$ & 6.68035 & -1.22717 & -1.54629 \\
$\mathrm{H}$ & 7.96144 & -0.70596 & -3.305 \\
$\mathrm{H}$ & 9.63362 & -0.40469 & -2.77126 \\
$\mathrm{H}$ & 8.6116 & 0.95295 & -3.23123 \\
$\mathrm{H}$ & -1.30415 & -1.22455 & 2.98518 \\
$\mathrm{H}$ & 0.45399 & -1.4982 & 2.9291 \\
$\mathrm{H}$ & 0.27302 & -0.12553 & 4.93031 \\
$\mathrm{H}$ & -1.03285 & 0.84743 & 4.21144 \\
$\mathrm{H}$ & 0.90237 & 2.27069 & 3.82258 \\
$\mathrm{H}$ & 1.91622 & 0.88302 & 3.39723 \\
$\mathrm{H}$ & 1.29075 & 1.39856 & 1.17909 \\
$\mathrm{H}$ & 0.71357 & 3.04068 & 0.03142 \\
$\mathrm{H}$ & -0.15036 & 4.25331 & -1.91975 \\
$\mathrm{H}$ & 0.82735 & 5.38018 & -0.95859 \\
$\mathrm{H}$ & -0.77057 & 5.87734 & -1.55286 \\
$\mathrm{H}$ & 0.176 & 5.9574 & 1.39389 \\
$\mathrm{H}$ & -1.45163 & 6.47157 & 0.87368 \\
$\mathrm{H}$ & -1.27723 & 5.24656 & 2.13995 \\
$\mathrm{H}$ & -1.78746 & 2.20716 & -0.58421 \\
$\mathrm{H}$ & -4.55722 & 3.2186 & -0.89197 \\
$\mathrm{H}$ & -3.81155 & 1.97531 & 3.1905 \\
$\mathrm{H}$ & -5.34872 & 2.89007 & 3.329 \\
$\mathrm{H}$ & -5.3725 & 1.1545 & 2.86889 \\
$\mathrm{H}$ & -3.62563 & 1.90741 & -2.84027 \\
$\mathrm{H}$ & -3.37424 & 0.5268 & -1.7736 \\
$\mathrm{H}$ & -5.98171 & 2.7297 & -3.28817 \\
$\mathrm{H}$ & -8.31652 & 2.03232 & -3.70503 \\
$\mathrm{H}$ & -9.12626 & -0.17187 & -2.88239 \\
$\mathrm{H}$ & -7.57598 & -1.66228 & -1.63567 \\
$\mathrm{H}$ & -5.24619 & -0.95418 & -1.20457 \\
& & & \\
\hline
\end{tabular}




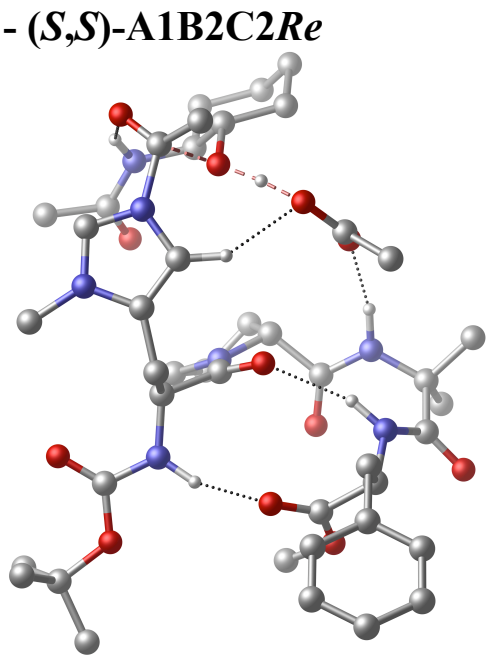

$\mathrm{N} \quad-1.85178 \quad 2.27775 \quad-0.47124$

$\begin{array}{llll}\text { C } & -0.5498 & 1.91858 & 0.05703\end{array}$

$\begin{array}{llll}\text { C } & -0.48284 & 2.02021 & 1.60549\end{array}$

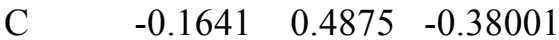

$\begin{array}{llll}\text { C } & 0.93474 & 2.05633 & 2.07295\end{array}$

$\begin{array}{llll}\mathrm{O} & -0.39526 & -0.47724 & 0.36617\end{array}$

$\begin{array}{llll}\mathrm{N} & 1.6987 & 3.22858 & 2.06884\end{array}$

$\begin{array}{llll}\text { C } & 1.79469 & 1.04196 & 2.39593\end{array}$

$\begin{array}{llll}\text { C } & 2.96369 & 2.91493 & 2.37704\end{array}$

$\begin{array}{llll}\mathrm{N} & 3.04608 & 1.59978 & 2.57751\end{array}$

$\mathrm{N} \quad 0.45884 \quad 0.36217 \quad-1.56346$

$\begin{array}{lllll}\mathrm{C} & & 0.65984 & 1.41041 & -2.58684\end{array}$

$\begin{array}{llll}\text { C } & 0.97179 & -0.95244 & -1.99221\end{array}$

$\begin{array}{lllll}\mathrm{C} & 0.94678 & 0.59944 & -3.85531\end{array}$

$\begin{array}{lllll}\text { C } & 1.70369 & -0.62221 & -3.31311\end{array}$

$\begin{array}{llll}\text { C } & 1.1981 & 4.58764 & 1.836\end{array}$

C $\quad-0.95123 \quad-4.26588-1.77879$

$\mathrm{N} \quad 0.00178 \quad-3.15551 \quad-1.65499$

C $\quad-2.33075 \quad-3.9321 \quad-1.14463$

O $\quad-3.31648 \quad-4.60566-1.41747$

C $\quad-0.37671-5.46894-1.00618$

C $\quad-1.16671-4.6521 \quad-3.25305$

C $\quad-2.17159 \quad 3.59596 \quad-0.60401$

O $\quad-1.38716 \quad 4.51769-0.37961$

O $\quad-3.44607 \quad 3.72548 \quad-1.02567$

$\begin{array}{llll}\text { C } & -4.04564 & 5.04917 & -1.26911\end{array}$

C $\quad \begin{array}{lllll}\text { C } & -3.28813 & 5.76624 & -2.39069\end{array}$

$\begin{array}{lllll}\text { C } & -5.46568 & 4.69033 & -1.71258\end{array}$

$\begin{array}{llll}\text { C } & -4.07211 & 5.8623 & 0.02923\end{array}$

C $\quad-6.64963 \quad-0.2026 \quad 3.06403$

$\begin{array}{llll}\text { C } & -5.38292 & -0.50817 & 2.56404\end{array}$

C $\quad-7.55884 \quad-1.2228 \quad 3.34612$

$\begin{array}{llll}\text { C } & -5.00187 & -1.83773 & 2.33958\end{array}$

$\begin{array}{llll}\text { C } & -7.19253 & -2.55154 & 3.12439\end{array}$

C $\quad-3.63513 \quad-2.18349 \quad 1.78368$

$\begin{array}{llll}\text { C } & -5.92514 & -2.85346 & 2.62664\end{array}$

$\begin{array}{llll}\text { C } & -3.63014 & -2.39432 & 0.25994\end{array}$
C

$\mathrm{O}$

$\mathrm{O}$

C

$\mathrm{O}$

C

C

C

O

C

C

C

C

C

C

O

$\mathrm{N}$

C

O

C

C

O

O

C

$\mathrm{H}$

$\mathrm{H}$

$\mathrm{H}$

$\mathrm{H}$

$\mathrm{H}$

$\mathrm{H}$

$\mathrm{H}$

$\mathrm{H}$

$\mathrm{H}$

$\mathrm{H}$

$\mathrm{H}$

$\mathrm{H}$

$\mathrm{H}$

$\mathrm{H}$

$\mathrm{H}$

$\mathrm{H}$

$\mathrm{H}$

$\mathrm{H}$

H

$\mathrm{H}$

$\mathrm{H}$

$\mathrm{H}$

H

$\mathrm{H}$

$\mathrm{H}$

$\mathrm{H}$

$\mathrm{H}$

$\mathrm{H}$

H $\begin{array}{llll}-2.35341 & -2.92509 & -0.21193\end{array}$

$\begin{array}{llll}-3.85463 & -1.09248 & -0.51447\end{array}$

$\begin{array}{llll}-3.54955 & 0.01402 & -0.09509\end{array}$

$\begin{array}{llll}-4.33307 & -1.3271 & -1.73449\end{array}$

$\begin{array}{llll}-4.28104 & -0.23415 & -2.6731\end{array}$

$\begin{array}{llll}-1.18431 & -1.61402 & -2.87151\end{array}$

$\begin{array}{llll}-0.18961 & -1.93465 & -2.21502\end{array}$

$\begin{array}{llll}4.38755 & 0.94238 & 2.87349\end{array}$

$\begin{array}{llll}4.32744 & 0.00491 & 4.05661\end{array}$

$\begin{array}{llll}5.35893 & 1.63514 & 2.59514\end{array}$

$6.7738-1.97266-1.50561$

$6.76227-2.62862-0.11788$

$\begin{array}{llll}6.62583 & -0.44905 & -1.39518\end{array}$

$\begin{array}{llll}5.49706 & -2.24886 & 0.66478\end{array}$

$\begin{array}{llll}5.36294 & -0.05584 & -0.60591\end{array}$

$\begin{array}{llll}5.33673 & -0.72609 & 0.78017\end{array}$

$\begin{array}{llll}4.13663 & -0.37208 & 1.45342\end{array}$

$\begin{array}{llll}5.26424 & 1.39333 & -0.44415\end{array}$

$\begin{array}{llll}4.58887 & 2.18673 & -1.30966\end{array}$

$\begin{array}{llll}4.04073 & 1.77286 & -2.33405\end{array}$

$\begin{array}{llll}1.92377 & -2.84961 & 1.5569\end{array}$

$\begin{array}{llll}0.85726 & -3.65227 & 2.29066\end{array}$

$\begin{array}{llll}2.11747 & -3.03235 & 0.34076\end{array}$

$\begin{array}{llll}2.57558 & -2.00798 & 2.29163\end{array}$

$\begin{array}{lll}4.56569 & 3.67 & -0.95861\end{array}$

$\begin{array}{llll}-5.44382 & 4.07659 & -2.61749\end{array}$

$\begin{array}{llll}-6.03378 & 5.60066 & -1.92528\end{array}$

$\begin{array}{llll}-5.98506 & 4.13119 & -0.92933\end{array}$

$\begin{array}{lll}-3.26004 & 5.14205 & -3.28913\end{array}$

$\begin{array}{llll}-3.80166 & 6.69972 & -2.6421\end{array}$

$\begin{array}{llll}-2.26656 & 5.99643 & -2.0874\end{array}$

$\begin{array}{llll}-4.63287 & 6.78864 & -0.13063\end{array}$

$\begin{array}{llll}-3.06285 & 6.11483 & 0.35495\end{array}$

$\begin{array}{llll}-4.57185 & 5.29616 & 0.82141\end{array}$

$\begin{array}{llll}-2.5935 & 1.57698 & -0.48307\end{array}$

$\begin{array}{llll}0.16464 & 2.62621 & -0.36975\end{array}$

$\begin{array}{lll}-1.01706 & 2.92123 & 1.91706\end{array}$

$\begin{array}{lll}-0.98622 & 1.14933 & 2.02789\end{array}$

$\begin{array}{lll}1.63385 & -0.02611 & 2.4423\end{array}$

$\begin{array}{llll}0.70055 & 4.96025 & 2.73539\end{array}$

$\begin{array}{llll}2.04379 & 5.23377 & 1.59882\end{array}$

$\begin{array}{llll}0.48769 & 4.59131 & 1.00581\end{array}$

$\begin{array}{llll}3.79449 & 3.5958 & 2.45595\end{array}$

$\begin{array}{lll}4.26051 & 0.60985 & 4.96858\end{array}$

$\begin{array}{llll}3.49 & -0.69063 \quad 4.01063\end{array}$

$\begin{array}{llll}5.26225 & -0.55673 & 4.08772\end{array}$

$\begin{array}{llll}-0.13007 & -3.37344 & 1.90873\end{array}$

$\begin{array}{llll}0.99862 & -4.71839 & 2.09228\end{array}$

$\begin{array}{llll}0.89041 & -3.47142 & 3.36584\end{array}$

$\begin{array}{llll}3.44766 & -1.22611 & 1.74303\end{array}$

$\begin{array}{llll}5.52051 & -2.68131 & 1.67164\end{array}$

$\begin{array}{llll}4.60785 & -2.65649 & 0.16938\end{array}$

$\begin{array}{llll}7.65359 & -2.30885 & 0.44101\end{array}$

$\begin{array}{llll}6.82671 & -3.71896 & -0.20857\end{array}$ 


$\begin{array}{llll}\mathrm{H} & 7.69726 & -2.22132 & -2.04147 \\ \mathrm{H} & 5.94587 & -2.37699 & -2.10383 \\ \mathrm{H} & 6.57849 & 0.01596 & -2.38543 \\ \mathrm{H} & 7.50447 & -0.02799 & -0.88651 \\ \mathrm{H} & 5.55976 & 1.77342 & 0.44904 \\ \mathrm{H} & 5.04919 & 3.90341 & -0.00582 \\ \mathrm{H} & 3.52806 & 4.01567 & -0.94049 \\ \mathrm{H} & 5.07361 & 4.22411 & -1.75303 \\ \mathrm{H} & -0.2297 & 2.03837 & -2.67119 \\ \mathrm{H} & 1.52982 & 2.02655 & -2.33157 \\ \mathrm{H} & 1.5318 & 1.17371 & -4.57771 \\ \mathrm{H} & 0.00746 & 0.28334 & -4.31611 \\ \mathrm{H} & 1.70515 & -1.47015 & -4.00249 \\ \mathrm{H} & 2.73786 & -0.34324 & -3.09254 \\ \mathrm{H} & 1.64645 & -1.34403 & -1.22679 \\ \mathrm{H} & 0.77937 & -3.25792 & -0.99388 \\ \mathrm{H} & -0.20768 & -5.22518 & 0.04563 \\ \mathrm{H} & 0.57657 & -5.778 & -1.44552 \\ \mathrm{H} & -1.07979 & -6.30255 & -1.06231 \\ \mathrm{H} & -0.21931 & -4.99357 & -3.68129 \\ \mathrm{H} & -1.90327 & -5.45441 & -3.31391 \\ \mathrm{H} & -1.53002 & -3.79704 & -3.8233 \\ \mathrm{H} & -1.54548 & -2.31456 & -0.12535 \\ \mathrm{H} & -4.38914 & -3.1199 & -0.04058 \\ \mathrm{H} & -3.2405 & -0.07193 & -2.96304 \\ \mathrm{H} & -4.86599 & -0.56177 & -3.53152 \\ \mathrm{H} & -4.70588 & 0.6723 & -2.23686 \\ \mathrm{H} & -3.27652 & -3.1161 & 2.2312 \\ \mathrm{H} & -2.91029 & -1.40105 & 2.02769 \\ \mathrm{H} & -5.64472 & -3.89128 & 2.4623 \\ \mathrm{H} & -7.89181 & -3.35371 & 3.34281 \\ \mathrm{H} & -8.54421 & -0.98539 & 3.73676 \\ \mathrm{H} & -6.926 & 0.83446 & 3.23392 \\ \mathrm{H} & -4.68229 & 0.28808 & 2.33255 \\ \mathrm{H} & 6.18126 & -0.31757 & 1.36135 \\ \mathrm{H} & 4.47678 & -0.3813 & -1.16071\end{array}$

\section{- $(R, R)-\mathrm{A} 2 \mathrm{~B} 2 \mathrm{C} 2 R e$}

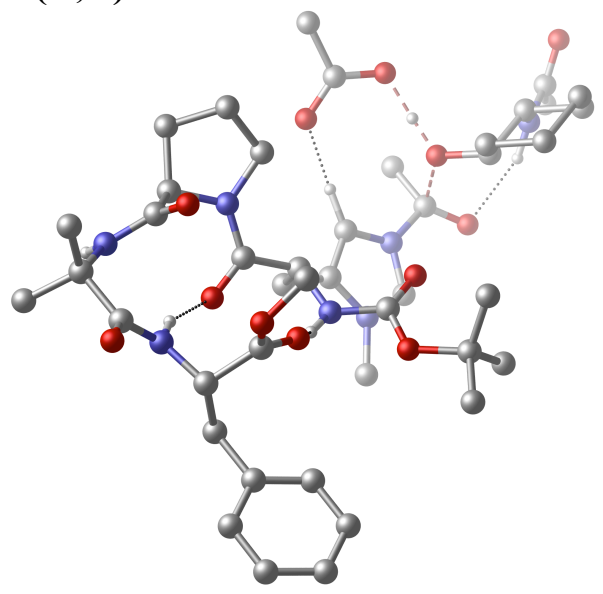

$\mathrm{N}$

$\begin{array}{llll}0.9026 & 0.92561 & -0.5547\end{array}$ $\begin{array}{llll}\text { C } & 0.38813 & -0.39353 & -0.89147\end{array}$

$\begin{array}{lllll}\text { C } & -0.16893 & -0.47562 & -2.35018\end{array}$

$\begin{array}{lllll}\text { C } & 1.51437 & -1.42759 & -0.80558\end{array}$

C $\quad \begin{array}{llll}-1.54403 & 0.09222 & -2.48901\end{array}$

O $\quad 2.59906-1.20628-1.36745$

$\mathrm{N} \quad \begin{array}{llll}-1.85072 & 1.41093 & -2.83128\end{array}$

$\begin{array}{lllll}\mathrm{C} & -2.73021 & -0.52449 & -2.21007\end{array}$

$\begin{array}{lllll}\mathrm{C} & -3.17765 & 1.57315 & -2.7475\end{array}$

$\mathrm{N} \quad \begin{array}{llll}\mathrm{C} & -3.72597 & 0.41343 & -2.38201\end{array}$

$\mathrm{N} \quad 1.23498 \quad-2.60788-0.21536$

$\begin{array}{llll}\text { C } & 0.07475 & -2.9492 & 0.64603\end{array}$

$\begin{array}{llll}\text { C } & 2.18701 & -3.71953 & -0.34711\end{array}$

$\begin{array}{llll}\text { C } & 0.5593 & -4.20293 & 1.38334\end{array}$

$\begin{array}{llll}\text { C } & 1.46651 & -4.89352 & 0.3543\end{array}$

$\begin{array}{lllll}\mathrm{C} & -0.90379 & 2.44605 & -3.24047\end{array}$

$\begin{array}{llll}\mathrm{C} & 6.02331 & -3.44117 & -0.07876\end{array}$

$\mathrm{N} \quad 4.61804 \quad-3.66018 \quad-0.45439$

$\begin{array}{llll}\text { C } & 6.34697 & -1.94015 & 0.16994\end{array}$

$\begin{array}{lllll}\text { O } & 7.38677 & -1.63261 & 0.73978\end{array}$

C $\quad 6.89013 \quad-3.89204-1.26958$

$\begin{array}{lllll}\text { C } & 6.38736 & -4.24635 & 1.17961\end{array}$

C $\quad \begin{array}{llll}\text { C } & 0.10735 & 1.80103 & 0.14123\end{array}$

$\begin{array}{llll}\mathrm{O} & -1.0907 & 1.638 & 0.33392\end{array}$

$\begin{array}{llll}\mathrm{O} & 0.83418 & 2.87829 & 0.51093\end{array}$

$\begin{array}{llll}\text { C } & 0.269 & 3.9117 & 1.39722\end{array}$

$\begin{array}{llll}\text { C } & -0.15091 & 3.28137 & 2.72837\end{array}$

$\begin{array}{llll}\text { C } & 1.4539 & 4.85947 & 1.5945\end{array}$

$\begin{array}{lllll}\text { C } & -0.89229 & 4.62888 & 0.70144\end{array}$

$\begin{array}{lllll}\text { C } & 5.07026 & 5.00008 & -0.43174\end{array}$

$\begin{array}{lllll}\mathrm{C} & 4.73788 & 3.66482 & -0.66803\end{array}$

$\begin{array}{lllll}\text { C } & 6.39289 & 5.42892 & -0.54736\end{array}$

$\begin{array}{llll}\text { C } & 5.72483 & 2.73647 & -1.02658\end{array}$

$\begin{array}{lllll}\text { C } & & 7.38463 & 4.51263 & -0.90159\end{array}$

$\begin{array}{llll}\text { C } & 5.39037 & 1.27854 & -1.27069\end{array}$

$\begin{array}{llll}\text { C } & 7.05046 & 3.1797 & -1.13868\end{array}$

$\begin{array}{llll}\text { C } & 5.60828 & 0.39152 & -0.03272\end{array}$

N $\quad 5.47833 \quad-1.02704 \quad-0.35658$

$\begin{array}{llll}\text { C } & 4.59015 & 0.67008 & 1.07776\end{array}$

$\begin{array}{lllll}\mathrm{O} & 3.44164 & 1.03784 & 0.89929\end{array}$

$\begin{array}{lllll}\mathrm{O} & 5.09064 & 0.36891 & 2.27876\end{array}$

C $\quad 4.14907 \quad 0.33883 \quad 3.36835$

$\begin{array}{lllll}\text { O } & 3.59189 & -2.93859 & 1.46442\end{array}$

$\begin{array}{llll}\text { C } & 3.53241 & -3.38393 & 0.32314\end{array}$

$\begin{array}{lllll}\text { C } & -5.20386 & 0.25938 & -2.16025\end{array}$

C $\quad-5.74555 \quad-1.07098 \quad-2.61971$

O $\quad-5.83078 \quad 1.30903 \quad-2.14897$

$\begin{array}{llll}\text { C } & -6.06741 & 1.14525 & 3.57405\end{array}$

$\begin{array}{llll}\text { C } & -4.62934 & 1.38132 & 3.09248\end{array}$

$\begin{array}{llll}\text { C } & -7.06377 & 1.28121 & 2.41552\end{array}$

$\begin{array}{lllll}\text { C } & -4.27428 & 0.44547 & 1.92874\end{array}$

$\begin{array}{lllll}\mathrm{C} & & -6.72276 & 0.33101 & 1.24973\end{array}$

$\begin{array}{lllll}\text { C } & -5.27531 & 0.55308 & 0.76676\end{array}$

$\begin{array}{lllll}\mathrm{O} & & -4.90377 & -0.33862 & -0.26904\end{array}$

$\begin{array}{lllll}\mathrm{N} & & -7.64534 & 0.50722 & 0.12929\end{array}$ 


\begin{tabular}{|c|c|c|}
\hline $\mathrm{C}$ & $\begin{array}{ll}-8.92685 & 0.05131\end{array}$ & 0.15888 \\
\hline $\mathrm{O}$ & $-9.41688-0.52096$ & 1.12947 \\
\hline $\mathrm{C}$ & $\begin{array}{lll}-3.89081 & -3.41483\end{array}$ & 0.07317 \\
\hline $\mathrm{C}$ & $\begin{array}{lll}-3.80451 & -4.75732\end{array}$ & 0.79578 \\
\hline $\mathrm{O}$ & $-3.03996-3.14189$ & -0.80717 \\
\hline $\mathrm{O}$ & $-4.85576-2.66377$ & 0.45301 \\
\hline $\mathrm{C}$ & $-9.73602 \quad 0.30436$ & -1.10906 \\
\hline $\mathrm{H}$ & $2.30777 \quad 4.32372$ & 2.01723 \\
\hline $\mathrm{H}$ & $1.17709 \quad 5.67228$ & 2.27219 \\
\hline $\mathrm{H}$ & $1.7605 \quad 5.29609$ & 0.63921 \\
\hline $\mathrm{H}$ & $0.68853 \quad 2.7332$ & 3.16694 \\
\hline $\mathrm{H}$ & $-0.44857 \quad 4.06724$ & 3.42963 \\
\hline $\mathrm{H}$ & $\begin{array}{ll}-0.99001 & 2.59802\end{array}$ & 2.5945 \\
\hline $\mathrm{H}$ & $-1.22888 \quad 5.46722$ & 1.31965 \\
\hline $\mathrm{H}$ & $-1.73026 \quad 3.95052$ & 0.5414 \\
\hline $\mathrm{H}$ & $-0.56534 \quad 5.0328$ & -0.26255 \\
\hline $\mathrm{H}$ & 1.906151 .00862 & -0.39989 \\
\hline $\mathrm{H}$ & $-0.43202-0.63034$ & -0.21245 \\
\hline $\mathrm{H}$ & $\begin{array}{lll}0.55266 & -0.00489\end{array}$ & -3.02332 \\
\hline $\mathrm{H}$ & $-0.23211-1.53045$ & -2.63551 \\
\hline $\mathrm{H}$ & $-2.92041-1.52074$ & -1.80099 \\
\hline $\mathrm{H}$ & $-0.529 \quad 2.23913$ & -4.24642 \\
\hline $\mathrm{H}$ & $-1.41319 \quad 3.40969$ & -3.23782 \\
\hline $\mathrm{H}$ & $-0.0686 \quad 2.47627$ & -2.54078 \\
\hline $\mathrm{H}$ & $-3.7324 \quad 2.47566$ & -2.94322 \\
\hline $\mathrm{H}$ & $-5.85362-1.03843$ & -3.71052 \\
\hline $\mathrm{H}$ & $-5.10762-1.90938$ & -2.34156 \\
\hline $\mathrm{H}$ & $-6.72836-1.20597$ & -2.16915 \\
\hline $\mathrm{H}$ & $-4.79412 \quad-5.21851$ & 0.84988 \\
\hline $\mathrm{H}$ & $-3.10437 \quad-5.4274$ & 0.29342 \\
\hline $\mathrm{H}$ & $-3.47284-4.59338$ & 1.82733 \\
\hline $\mathrm{H}$ & $-4.88323-1.40759$ & 0.02941 \\
\hline $\mathrm{H}$ & $-4.27416-0.59598$ & 2.27588 \\
\hline $\mathrm{H}$ & $-3.27332 \quad 0.65746$ & 1.539 \\
\hline $\mathrm{H}$ & $-3.91785 \quad 1.24031$ & 3.91546 \\
\hline $\mathrm{H}$ & -4.52433 & 2.76674 \\
\hline $\mathrm{H}$ & $-6.14504 \quad 0.13724$ & 4.0044 \\
\hline $\mathrm{H}$ & $-6.3257 \quad 1.84718$ & 4.37607 \\
\hline $\mathrm{H}$ & $-7.05314 \quad 2.31405$ & 2.03856 \\
\hline $\mathrm{H}$ & $-8.08429 \quad 1.06633$ & 2.74366 \\
\hline $\mathrm{H}$ & $\begin{array}{ll}-6.8192 & -0.70427\end{array}$ & 1.60083 \\
\hline $\mathrm{H}$ & $-5.21806 \quad 1.56808$ & 0.33931 \\
\hline $\mathrm{H}$ & $\begin{array}{ll}-7.29064 & 0.95656\end{array}$ & -0.70717 \\
\hline $\mathrm{H}$ & $-9.1586 \quad 0.76612$ & -1.91491 \\
\hline $\mathrm{H}$ & $\begin{array}{ll}-10.58433 & 0.9501\end{array}$ & $7-0.86407$ \\
\hline $\mathrm{H}$ & $\begin{array}{lll}-10.14526 & -0.6477\end{array}$ & $5-1.45766$ \\
\hline $\mathrm{H}$ & $-0.14166 \quad-2.12871$ & 1.33375 \\
\hline $\mathrm{H}$ & $-0.8195 \quad-3.14747$ & 0.04239 \\
\hline $\mathrm{H}$ & $-0.27332-4.83208$ & 1.70675 \\
\hline $\mathrm{H}$ & $1.14978-3.9161$ & 2.25724 \\
\hline $\mathrm{H}$ & $2.17077-5.59705$ & 0.80487 \\
\hline $\mathrm{H}$ & $\begin{array}{lll}0.86695 & -5.43905\end{array}$ & -0.3818 \\
\hline $\mathrm{H}$ & $\begin{array}{lll}2.3475 & -3.922 & -\end{array}$ & 1.41228 \\
\hline $\mathrm{H}$ & $4.44008-3.90985$ & -1.41623 \\
\hline
\end{tabular}

$\begin{array}{llll}\mathrm{H} & 6.63506 & -3.33689 & -2.17941 \\ \mathrm{H} & 6.75657 & -4.96246 & -1.45743 \\ \mathrm{H} & 7.94078 & -3.70467 & -1.04152 \\ \mathrm{H} & 6.27486 & -5.31589 & 0.97713 \\ \mathrm{H} & 7.42079 & -4.03404 & 1.45558 \\ \mathrm{H} & 5.73792 & -3.97014 & 2.01075 \\ \mathrm{H} & 4.57862 & -1.32702 & -0.72266 \\ \mathrm{H} & 6.61294 & 0.52254 & 0.3738 \\ \mathrm{H} & 3.42941 & -0.467 & 3.20648 \\ \mathrm{H} & 4.74375 & 0.14619 & 4.2602 \\ \mathrm{H} & 3.62458 & 1.29308 & 3.45076 \\ \mathrm{H} & 6.03156 & 0.87816 & -2.06282 \\ \mathrm{H} & 4.35432 & 1.16682 & -1.60446 \\ \mathrm{H} & 7.82733 & 2.47213 & -1.41924 \\ \mathrm{H} & 8.41733 & 4.83587 & -0.99681 \\ \mathrm{H} & 6.64936 & 6.46842 & -0.36447 \\ \mathrm{H} & 4.29263 & 5.70695 & -0.15591 \\ \mathrm{H} & 3.70772 & 3.33771 & -0.56289\end{array}$

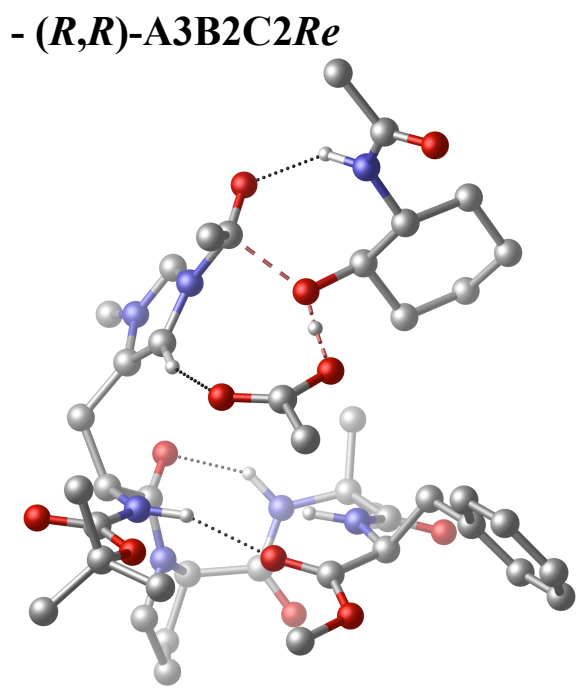

$\begin{array}{llll}\mathrm{N} & 2.32397 & -2.25569 & 0.42763 \\ \mathrm{C} & 2.78446 & -2.57218 & -0.91224 \\ \mathrm{C} & 1.81776 & -3.49698 & -1.68999 \\ \mathrm{C} & 3.19493 & -1.33285 & -1.72545 \\ \mathrm{C} & 0.43536 & -3.01029 & -2.0069 \\ \mathrm{O} & 2.63543 & -1.06973 & -2.80499 \\ \mathrm{~N} & -0.02075 & -2.85317 & -3.32043 \\ \mathrm{C} & -0.6544 & -2.80178 & -1.19984 \\ \mathrm{C} & -1.33 & -2.57969 & -3.2933 \\ \mathrm{~N} & -1.73505 & -2.5478 & -2.02596 \\ \mathrm{~N} & 4.24217 & -0.60508 & -1.2759 \\ \mathrm{C} & 5.02739 & -0.85342 & -0.04352 \\ \mathrm{C} & 4.90789 & 0.37905 & -2.16698 \\ \mathrm{C} & 6.15377 & 0.18691 & -0.11657 \\ \mathrm{C} & 6.33943 & 0.42671 & -1.62206 \\ \mathrm{C} & 0.7773 & -2.96233 & -4.54028 \\ \mathrm{C} & 2.23357 & 3.06775 & -2.88023 \\ \mathrm{~N} & 3.0435 & 1.83986 & -2.78096\end{array}$




\begin{tabular}{|c|c|c|c|}
\hline $\mathrm{C}$ & 1.81425 & 3.57465 & -1.46963 \\
\hline $\mathrm{O}$ & 1.60816 & 4.76095 & -1.24136 \\
\hline $\mathrm{C}$ & 0.94512 & 2.70597 & -3.64319 \\
\hline $\mathrm{C}$ & 2.99543 & 4.18007 & -3.61507 \\
\hline $\mathrm{C}$ & 2.42931 & -3.22539 & 1.39204 \\
\hline $\mathrm{O}$ & 2.80357 & -4.36531 & 1.14126 \\
\hline $\mathrm{O}$ & 2.09344 & -2.74121 & 2.60149 \\
\hline $\mathrm{C}$ & 1.82986 & -3.67082 & 3.72654 \\
\hline $\mathrm{C}$ & 3.11356 & -4.41221 & 4.11079 \\
\hline $\mathrm{C}$ & 1.39855 & -2.72518 & 4.84796 \\
\hline $\mathrm{C}$ & 0.68381 & -4.61153 & 3.3436 \\
\hline $\mathrm{C}$ & -2.00032 & 3.32778 & 4.39928 \\
\hline $\mathrm{C}$ & -1.57314 & 2.86243 & 3.15343 \\
\hline $\mathrm{C}$ & -1.53779 & 4.54902 & 4.88967 \\
\hline $\mathrm{C}$ & -0.67926 & 3.61532 & 2.38106 \\
\hline $\mathrm{C}$ & -0.65433 & 5.31084 & 4.12123 \\
\hline $\mathrm{C}$ & -0.24157 & 3.11815 & 1.02162 \\
\hline $\mathrm{C}$ & -0.23269 & 4.84936 & 2.87517 \\
\hline $\mathrm{C}$ & 1.28734 & 2.88114 & 0.83261 \\
\hline $\mathrm{N}$ & 1.60221 & 2.59498 & -0.5526 \\
\hline $\mathrm{C}$ & 1.78826 & 1.69736 & 1.64632 \\
\hline $\mathrm{O}$ & 2.08846 & 0.61991 & 1.15038 \\
\hline $\mathrm{O}$ & 1.84711 & 1.96129 & 2.9464 \\
\hline $\mathrm{C}$ & 2.24939 & 0.87761 & 3.81106 \\
\hline $\mathrm{O}$ & 4.73316 & 2.71473 & -1.51472 \\
\hline $\mathrm{C}$ & 4.22657 & 1.77129 & -2.11341 \\
\hline $\mathrm{C}$ & -3.20644 & -2.37738 & -1.69814 \\
\hline $\mathrm{C}$ & -3.61873 & -3.14398 & -0.46708 \\
\hline $\mathrm{O}$ & -3.91638 & -2.20786 & -2.68458 \\
\hline $\mathrm{C}$ & -4.94997 & 3.12017 & -1.37707 \\
\hline $\mathrm{C}$ & -3.55195 & 2.93276 & -1.97907 \\
\hline $\mathrm{C}$ & -5.77764 & 1.83733 & -1.51443 \\
\hline $\mathrm{C}$ & -2.84926 & 1.72855 & -1.33947 \\
\hline C & -5.08055 & 0.61096 & -0.88506 \\
\hline $\mathrm{C}$ & -3.66037 & 0.42986 & -1.46244 \\
\hline $\mathrm{O}$ & -2.93141 & -0.63492 & -0.86665 \\
\hline $\mathrm{N}$ & -5.86833 & -0.59951 & -1.10964 \\
\hline C & -7.0225 & -0.85485 & -0.4339 \\
\hline $\mathrm{O}$ & -7.4774 & -0.10638 & 0.42742 \\
\hline $\mathrm{C}$ & -2.01246 & -1.22095 & 2.22411 \\
\hline $\mathrm{C}$ & -2.09506 & -0.99529 & 3.73125 \\
\hline $\mathrm{O}$ & -1.42019 & -2.22754 & 1.78099 \\
\hline $\mathrm{O}$ & -2.58349 & -0.308 & 1.51733 \\
\hline $\mathrm{C}$ & $-7.73-$ & $2.14935-$ & 0.81944 \\
\hline $\mathrm{H}$ & 2.20601 & -2.0326 & 5.10544 \\
\hline $\mathrm{H}$ & 1.14732 & -3.30151 & 5.74315 \\
\hline $\mathrm{H}$ & 0.51941 & -2.15148 & 4.54666 \\
\hline $\mathrm{H}$ & 3.91811 & -3.69963 & 4.32025 \\
\hline $\mathrm{H}$ & 2.93572 & -4.99689 & 5.01937 \\
\hline $\mathrm{H}$ & 3.43394 & -5.08264 & 3.31433 \\
\hline $\mathrm{H}$ & 0.37778 & -5.1894 & 4.22188 \\
\hline $\mathrm{H}$ & 0.99542 & -5.31047 & 2.56544 \\
\hline $\mathrm{H}$ & -0.17078 & -4.02854 & 2.98592 \\
\hline $\mathrm{H}$ & 1.98966 & -1.33235 & 0.68222 \\
\hline
\end{tabular}

\begin{tabular}{|c|c|c|}
\hline $\mathrm{H}$ & $3.69243-3.17911$ & -0.78944 \\
\hline $\mathrm{H}$ & $2.31588-3.79824$ & -2.61494 \\
\hline $\mathrm{H}$ & $1.7356-4.39519$ & -1.07166 \\
\hline $\mathrm{H}$ & $\begin{array}{lll}-0.75341 & -2.76047\end{array}$ & -0.11842 \\
\hline $\mathrm{H}$ & $1.09024-3.99762$ & -4.69835 \\
\hline $\mathrm{H}$ & $0.16441-2.64523$ & -5.38445 \\
\hline $\mathrm{H}$ & $1.64845-2.31421$ & -4.44426 \\
\hline $\mathrm{H}$ & $-1.97938-2.42143$ & -4.13807 \\
\hline $\mathrm{H}$ & $-3.71162-4.20002$ & -0.74795 \\
\hline $\mathrm{H}$ & $-2.914 \quad-3.04257$ & 0.35859 \\
\hline $\mathrm{H}$ & $\begin{array}{ll}-4.59589 & -2.77676\end{array}$ & -0.15564 \\
\hline $\mathrm{H}$ & $-3.08925-0.63141$ & 4.00236 \\
\hline $\mathrm{H}$ & $-1.87166-1.91516$ & 4.27378 \\
\hline $\mathrm{H}$ & $-1.37557-0.22312$ & 4.02687 \\
\hline $\mathrm{H}$ & $-2.75081-0.52114$ & 0.2416 \\
\hline $\mathrm{H}$ & $-2.69209 \quad 1.92758$ & -0.27321 \\
\hline $\mathrm{H}$ & $-1.85984 \quad 1.56121$ & -1.78039 \\
\hline $\mathrm{H}$ & $-2.94476 \quad 3.83544$ & -1.84154 \\
\hline $\mathrm{H}$ & $\begin{array}{ll}-3.64035 & 2.77628\end{array}$ & -3.06391 \\
\hline $\mathrm{H}$ & $-4.8552 \quad 3.38478$ & -0.31507 \\
\hline $\mathrm{H}$ & $\begin{array}{ll}-5.46967 & 3.95549\end{array}$ & -1.86096 \\
\hline $\mathrm{H}$ & $-5.95226 \quad 1.6255$ & -2.57914 \\
\hline $\mathrm{H}$ & $-6.75781 \quad 1.94657$ & -1.04258 \\
\hline $\mathrm{H}$ & $\begin{array}{ll}-5.00413 & 0.76304\end{array}$ & 0.19864 \\
\hline $\mathrm{H}$ & $-3.76406 \quad 0.17127$ & -2.52808 \\
\hline $\mathrm{H}$ & $-5.54118-1.24249$ & -1.82246 \\
\hline $\mathrm{H}$ & $-7.18253-2.74945$ & -1.55154 \\
\hline $\mathrm{H}$ & $-8.71558-1.90458$ & -1.22609 \\
\hline $\mathrm{H}$ & $\begin{array}{lll}-7.89146 & -2.74237\end{array}$ & 0.08478 \\
\hline $\mathrm{H}$ & $4.39804-0.73786$ & 0.83916 \\
\hline $\mathrm{H}$ & $5.42428-1.87662$ & -0.05514 \\
\hline $\mathrm{H}$ & $7.06175-0.16254$ & 0.38104 \\
\hline $\mathrm{H}$ & $\begin{array}{lll}5.83591 & 1.11972\end{array}$ & 0.35585 \\
\hline $\mathrm{H}$ & $6.81404 \quad 1.38427$ & -1.83742 \\
\hline $\mathrm{H}$ & $6.92963-0.37671$ & -2.07596 \\
\hline $\mathrm{H}$ & $4.84771 \quad-0.00045$ & -3.19159 \\
\hline $\mathrm{H}$ & $2.68789 \quad 0.96974$ & -3.16797 \\
\hline $\mathrm{H}$ & $0.39945 \quad 1.8987$ & -3.14428 \\
\hline $\mathrm{H}$ & $1.18356 \quad 2.39205$ & -4.66499 \\
\hline $\mathrm{H}$ & $0.29166 \quad 3.57961$ & -3.69548 \\
\hline $\mathrm{H}$ & 3.209813 .8645 & -4.6407 \\
\hline $\mathrm{H}$ & $2.38916 \quad 5.0869$ & -3.63326 \\
\hline $\mathrm{H}$ & $3.93173 \quad 4.40403$ & -3.1035 \\
\hline $\mathrm{H}$ & $1.87256 \quad 1.64415$ & -0.75966 \\
\hline $\mathrm{H}$ & $1.82625 \quad 3.78146$ & 1.14452 \\
\hline $\mathrm{H}$ & $3.33688 \quad 0.7687$ & 3.78001 \\
\hline $\mathrm{H}$ & $1.92772 \quad 1.16902$ & 4.80966 \\
\hline $\mathrm{H}$ & $1.78413-0.05719$ & 3.49948 \\
\hline $\mathrm{H}$ & -0.501713 .85392 & 0.2532 \\
\hline $\mathrm{H}$ & $-0.76137 \quad 2.18795$ & 0.77657 \\
\hline $\mathrm{H}$ & $0.44037 \quad 5.45813$ & 2.2759 \\
\hline $\mathrm{H}$ & $-0.29988 \quad 6.26956$ & 4.48958 \\
\hline $\mathrm{H}$ & $-1.87018 \quad 4.9113$ & 5.85836 \\
\hline $\mathrm{H}$ & $-2.70067 \quad 2.73605$ & 4.98214 \\
\hline
\end{tabular}




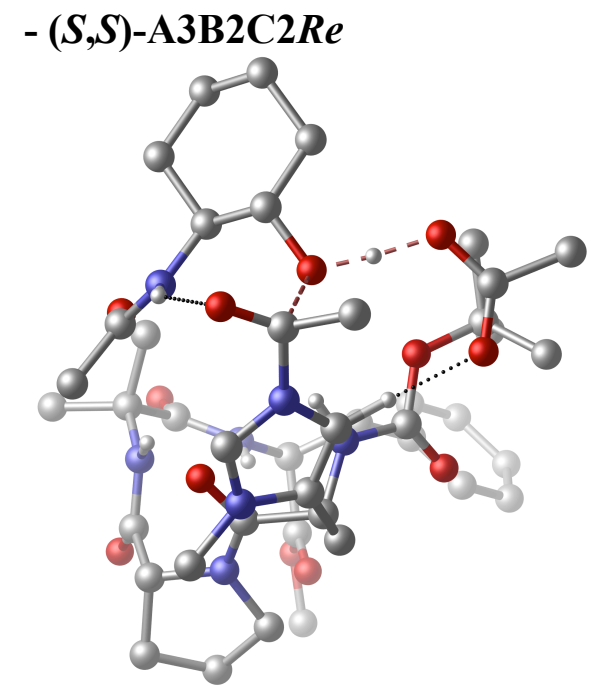

$\mathrm{N}$

C

$\mathrm{O}$

$\mathrm{O}$

C

$\mathrm{O}$

C

C

C

$\mathrm{O}$

C

$\mathrm{C}$

$\mathrm{C}$

$\mathrm{C}$

C

C

$\mathrm{O}$

$\mathrm{N}$

C

$\mathrm{O}$

C

C

$\mathrm{O}$

$\mathrm{O}$

C

$\mathrm{H}$

$\mathrm{H}$

$\mathrm{H}$

$\mathrm{H}$

$\mathrm{H}$

$\mathrm{H}$

$\mathrm{H}$

$\mathrm{H}$

$\mathrm{H}$

$\mathrm{H}$

$\mathrm{H}$

$\mathrm{H}$

$\mathrm{H}$

$\mathrm{H}$

H

$\mathrm{H}$

$\mathrm{H}$

$\mathrm{H}$

$\mathrm{H}$

$\mathrm{H}$

$\mathrm{H}$

$\mathrm{H}$

$\mathrm{H}$

$\mathrm{H}$

$\mathrm{H}$

$\mathrm{H}$

$\mathrm{H}$ $\begin{array}{lll}-4.62129 & 0.21691 & 1.10586\end{array}$

$\begin{array}{lll}-3.51373 & -0.68465 & 1.35667\end{array}$

$\begin{array}{lll}-5.36325 & -0.22707 & -0.15386\end{array}$

$\begin{array}{llll}-4.81353 & -0.47783 & -1.21138\end{array}$

$\begin{array}{llll}-6.68701 & -0.27996 & 0.03264\end{array}$

$-7.47144-0.63853-1.11953$

$\begin{array}{llll}-3.72958 & -3.74636 & 0.34295\end{array}$

$\begin{array}{lll}-2.55204 & -3.44558 & 0.1716\end{array}$

$\begin{array}{lll}5.70757 & -0.4682 & -0.97693\end{array}$

$\begin{array}{llll}6.47492 & 0.63786 & -1.65606\end{array}$

$\begin{array}{llll}6.2226 & -1.38462 & -0.34849\end{array}$

$\begin{array}{llll}4.52094 & 0.50971 & 4.59959\end{array}$

$\begin{array}{lll}5.55928 & 1.42379 & 3.93846\end{array}$

$\begin{array}{llll}4.36762 & -0.79322 & 3.80777\end{array}$

$\begin{array}{llll}5.19878 & 1.69652 & 2.47217\end{array}$

$\begin{array}{lll}4.01845 & -0.53986 & 2.32621\end{array}$

$\begin{array}{lll}5.04323 & 0.39885 & 1.65915\end{array}$

$\begin{array}{lll}4.67122 & 0.66652 & 0.30987\end{array}$

$\begin{array}{llll}3.9479 & -1.80571 & 1.59635\end{array}$

$\begin{array}{llll}2.88854 & -2.64427 & 1.70327\end{array}$

$\begin{array}{lll}1.8894 & -2.38164 & 2.37519\end{array}$

$\begin{array}{llll}4.45655 & 3.53251 & -1.2925\end{array}$

$\begin{array}{llll}4.70098 & 5.02837 & -1.45245\end{array}$

$\begin{array}{llll}3.93055 & 2.88874 & -2.22284\end{array}$

$\begin{array}{llll}4.82496 & 3.0469 & -0.15681\end{array}$

$\begin{array}{llll}2.98745 & -3.95446 & 0.93497\end{array}$

$\begin{array}{lll}1.0106 & 3.74874 & 1.95008\end{array}$

$\begin{array}{lll}1.26558 & 5.34627 & 1.22273\end{array}$

$\begin{array}{llll}2.30686 & 3.97953 & 0.76151\end{array}$

$\begin{array}{lll}-1.40443 & 3.72558 & 1.22306\end{array}$

$\begin{array}{lll}-1.22336 & 5.3285 & 0.49203\end{array}$

$\begin{array}{llll}-1.83157 & 3.97845 & -0.48588\end{array}$

$\begin{array}{llll}0.787 & 5.67635 & -1.25961\end{array}$

$\begin{array}{llll}-0.01532 & 4.39964 & -2.19584\end{array}$

$\begin{array}{llll}1.69171 & 4.22397 & -1.74086\end{array}$

$\begin{array}{llll}1.08509 & 0.22856 & -0.53\end{array}$

$\begin{array}{llll}-0.86587 & -0.04433 & -2.72881\end{array}$

$\begin{array}{llll}0.83015 & -1.34083 & -4.0542\end{array}$

$\begin{array}{llll}1.19929 & 0.3611 & -3.80986\end{array}$

$\begin{array}{llll}3.43343 & 1.01011 & -2.11604\end{array}$

$2.10572-3.38283-4.11708$

$\begin{array}{llll}3.13385 & -4.26729 & -2.97453\end{array}$

$\begin{array}{llll}1.53364 & -3.68743 & -2.44611\end{array}$

$\begin{array}{llll}4.83791 & -2.95903 & -1.74221\end{array}$

$\begin{array}{llll}7.07557 & 0.17955 & -2.45097\end{array}$

$\begin{array}{llll}5.82603 & 1.39929 & -2.08742\end{array}$

$\begin{array}{llll}7.15121 & 1.09264 & -0.93214\end{array}$

$\begin{array}{lll}5.69556 & 5.292 & -1.08349\end{array}$

$\begin{array}{llll}4.5901 & 5.3257 & -2.49616\end{array}$

$3.97454 \quad 5.58115 \quad-0.84728$

$\begin{array}{llll}4.70979 & 1.78846 & 0.05104\end{array}$

$\begin{array}{llll}5.94982 & 2.33059 & 1.99325\end{array}$

$\begin{array}{lll}4.25525 & 2.25417 & 2.42263\end{array}$ 


$\begin{array}{llll}\mathrm{H} & 6.54849 & 0.94721 & 3.99283 \\ \mathrm{H} & 5.64136 & 2.37342 & 4.48003 \\ \mathrm{H} & 4.80301 & 0.28786 & 5.6356 \\ \mathrm{H} & 3.5536 & 1.02921 & 4.64398 \\ \mathrm{H} & 3.58863 & -1.42904 & 4.2383 \\ \mathrm{H} & 5.30831 & -1.36032 & 3.84979 \\ \mathrm{H} & 4.75185 & -2.05552 & 1.03074 \\ \mathrm{H} & 3.95512 & -4.10835 & 0.44819 \\ \mathrm{H} & 2.18692 & -3.96877 & 0.19089 \\ \mathrm{H} & 2.81008 & -4.77899 & 1.6305 \\ \mathrm{H} & -2.92016 & -1.02934 & -2.63742 \\ \mathrm{H} & -1.98677 & -1.95442 & -3.84635 \\ \mathrm{H} & -3.95555 & -3.32282 & -3.80562 \\ \mathrm{H} & -4.34692 & -2.8162 & -2.1529 \\ \mathrm{H} & -3.47699 & -5.03215 & -1.73406 \\ \mathrm{H} & -2.21298 & -4.85399 & -2.97642 \\ \mathrm{H} & -0.93386 & -4.25986 & -1.0261 \\ \mathrm{H} & -0.7913 & -2.70687 & 0.91963 \\ \mathrm{H} & -0.93578 & -0.79319 & 2.64961 \\ \mathrm{H} & -0.25352 & -2.30378 & 3.25996 \\ \mathrm{H} & -1.51472 & -1.45353 & 4.1906 \\ \mathrm{H} & -1.72622 & -4.38648 & 3.38972 \\ \mathrm{H} & -3.03213 & -3.51005 & 4.22839 \\ \mathrm{H} & -3.37995 & -4.36748 & 2.72075 \\ \mathrm{H} & -2.85222 & -0.82566 & 0.60669 \\ \mathrm{H} & -5.29207 & 0.14367 & 1.96282 \\ \mathrm{H} & -7.21765 & -1.64729 & -1.45321 \\ \mathrm{H} & -8.50887 & -0.59458 & -0.79082 \\ \mathrm{H} & -7.29478 & 0.06681 & -1.93457 \\ \mathrm{H} & -3.57177 & 1.9265 & 1.84816 \\ \mathrm{H} & -3.38049 & 1.68014 & 0.11117 \\ \mathrm{H} & -5.87909 & 2.77155 & 2.69764 \\ \mathrm{H} & -7.68957 & 4.39259 & 2.24088 \\ \mathrm{H} & -8.05416 & 5.23988 & -0.06874 \\ \mathrm{H} & -6.58593 & 4.45162 & -1.91475 \\ \mathrm{H} & -4.77447 & 2.82488 & -1.45229 \\ \mathrm{H} & 6.01358 & -0.12762 & 1.64844 \\ \mathrm{H} & 3.02882 & -0.07168 & 2.26869 \\ & & & \\ & & & \end{array}$

